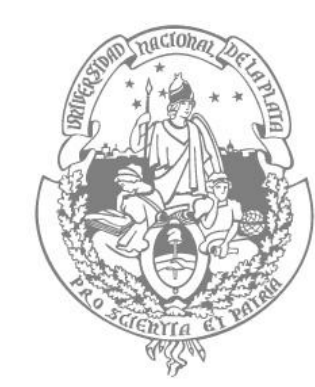

Facultad de Ciencias Naturales y Museo de La Plata Universidad Nacional de La Plata

\title{
LAS CACTÁCEAS Y SUS POLINIZADORES EN EL NOROESTE DE ARGENTINA: DISTRIBUCIÓN GEOGRÁFICA, CAMBIO CLIMÁTICO Y CONSERVACIÓN
}

\author{
Pablo Gorostiague
}

Tesis para obtener el título de Doctor en Ciencias Naturales

Director: Dr. Pablo Ortega-Baes

Co-Directora: Dra. Liliana Katinas 


\section{AGRADECIMIENTOS}

A mis papás y hermanos por enseñarme todo lo que se necesita saber para conseguir las cosas que uno quiere, y disfrutarlas.

A mi director Pablo Ortega por transmitirme un poco de su gran pasión, por acompañarme cerca desde que empecé, y por generar oportunidades para los que se interesan por la ciencia.

A mi co-directora Liliana Katinas, por su ayuda, buena predisposición y confianza. A mis compañeros de laboratorio por la compañía, la gran ayuda, y el maravilloso clima que construyen.

A mis amigos por estar siempre.

A los evaluadores de este trabajo, Dra. Lorena Ashworth, Dra. María Lujan Luna y Dr. Gonzalo Márquez.

Al Consejo Nacional de Investigaciones Científicas y Técnicas (CONICET) por la beca doctoral, al Proyecto PICT 2011/149, PIP 2011/112 y Proyecto 2060 del CIUNSa.

Al Posgrado de la Facultad de Ciencias Naturales y Museo de la Universidad Nacional de la Plata.

A la Universidad Nacional de Salta. Confío en el poder de las instituciones libres, democráticas e inclusivas para transformar el medio en beneficio de todos. 


\section{ÍNDICE}

Resumen

Abstract

Introducción General

Capítulo I: Biología reproductiva de las cactáceas de la tribu Trichocereeae en el Noroeste de Argentina 17

Introducción 18

Materiales y Métodos 22

Resultados 25

Discusión 28

Referencias Bibliográficas 33

Tablas 37

Figuras 41

Capítulo II: ¿Qué tan especializados son los sistemas de polinización en la familia Cactaceae?: el caso de la polinización por aves 53

Introducción 54

Materiales y Métodos 56

Resultados 61

Discusión 65

Referencias Bibliográficas 72

Tablas 79

Figuras 84

Capítulo III: Efecto del cambio climático sobre la distribución geográfica de 12 especies de cactáceas del Noroeste de Argentina y el acople espacial con sus polinizadores 89

Introducción 90

Materiales y Métodos 94

Resultados 98

Discusión 100

Referencias Bibliográficas 105 
Tablas

Figuras

Capítulo IV: Estrategias de conservación in situ para 12 especies de cactáceas del Noroeste de Argentina ………................................................. 138

Introducción 139

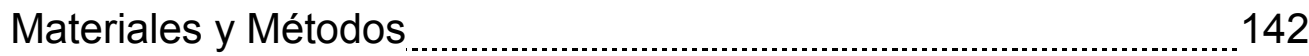

Resultados

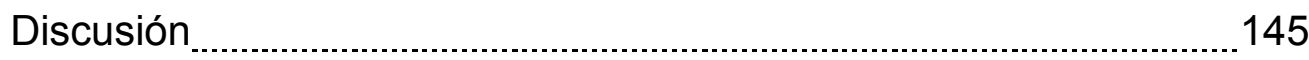

Referencias Bibliográficas …………………………............................ 148

Figuras

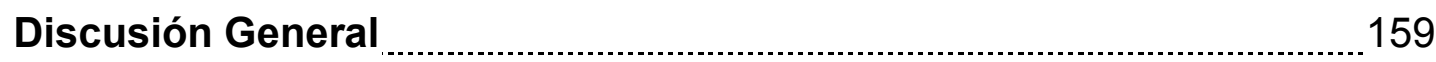

Publicaciones 


\section{RESUMEN}

Las cactáceas son un grupo de especial interés para conservación ya que están amenazadas, principalmente por la pérdida y degradación del hábitat y la colecta ilegal y potencialmente por el cambio climático. Las especies de esta familia establecen numerosas interacciones mutualistas con otros organismos, asociadas a la polinización de sus flores, la dispersión de sus semillas y al establecimiento de nuevos individuos. Particularmente, la polinización se ha indicado como un proceso fundamental en el ciclo de vida de estas plantas, dado que muchas especies dependen de este mutualismo para su reproducción. En esta tesis, se estudió la biología reproductiva de ocho especies de la tribu Trichocereeae, a fin de conocer el grado de dependencia de los polinizadores que tienen las cactáceas de la tribu Trichocereeae del noroeste de Argentina para la producción de frutos y semillas, evaluando para 12 especies los efectos potenciales del cambio climático sobre el acople espacial entre las especies de este grupo de plantas y sus mutualistas y generando un esquema de conservación para este grupo de especies y sus polinizadores. Los resultados obtenidos indicaron que las ocho especies estudiadas en la primera parte del análisis (Cleistocactus baumannii, C. smaragdiflorus, Denmoza rhodacantha, Echinopsis albispinosa, E. haematantha, E. leucantha, E. thionantha y Gymnocalycium saglionis) son auto-incompatibles, y por lo tanto necesitan de sus polinizadores para la producción de frutos y semillas. Entre los polinizadores de estas especies, se registraron abejas, aves, mariposas nocturnas y avispas, presentando, en la mayoría de los casos, sistemas de polinización generalistas, al menos funcionalmente. A partir de la información disponible para toda la familia, se determinó que la especialización fenotípica a la polinización por aves es común en esta familia de plantas; sin embargo, ésta no es predictiva de la especialización a nivel funcional, ya que en casi todas las especies de cactáceas polinizadas por aves se registraron adicionalmente otros grupos de polinizadores que incluyen abejas, murciélagos, mariposas nocturnas, entre otros. De acuerdo a los resultados, se registró el primer caso de un sistema de polinización especializado a los tres niveles (fenotípico, funcional y ecológico) para la familia: el sistema Denmoza rhodacantha-Oreotrochilus leucopleurus. Los efectos del cambio climático sobre las especies de cactáceas estudiadas fueron 
variables, siendo positivos para algunas especies (incremento del rango geográfico en $E$. atacamensis, E. leucantha y $G$. saglionis) y negativos para otras (reducción del rango geográfico en $C$. baumannii, C. smaragdiflorus, $E$. albispinosa, E. ancistrophora, E. haematantha, E. leucantha, E. schickendantzii, E. terscheckii y $E$. thelegona). Los resultados obtenidos resaltaron la importancia de incorporar las interacciones bióticas en el rango geográfico de las especies, ya que las mismas pueden limitar en gran medida las áreas que ocupan las especies; sin embargo, en la mayoría de los casos, el cambio climático no generaría un aumento en el desacople espacial entre las cactáceas y sus polinizadores, a excepción de la especie que presenta un sistema de polinización especializado (Denmoza rhodacantha). En esta especie el desacople incrementaría bajo escenarios de cambio climático futuro. Las áreas prioritarias para la conservación seleccionadas con base en el rango geográfico acoplado tuvieron una congruencia muy baja ( $26 \%$ de áreas compartidas) con respecto a las áreas seleccionadas con base en la distribución sin considerar los polinizadores. De igual manera, las áreas seleccionadas bajo escenarios de cambio climático presentaron una baja congruencia espacial (entre un 12 y un 15\%, según el escenario utilizado) con las áreas seleccionadas para el presente. Con base en estos resultados, se proponen como prioritarias las áreas que son comunes a todos los escenarios, tanto actuales como futuros. Las mismas fueron cinco y estuvieron distribuidas en las provincias de Salta y La Rioja. Estas áreas son de importancia para generar estrategias de conservación a largo plazo para las cactáceas y sus mutualistas polinizadores. En función de los resultados de la presente tesis, futuros estudios deberían incorporar las otras interacciones positivas que las cactáceas establecen con dispersores de semillas y plantas nodrizas en la evaluación del impacto de amenazas y en la definición de estrategias de conservación in situ. El enfoque y las ideas puestas a prueba en la presente tesis podrían ser utilizados en estudios similares con otras especies de plantas que tienen prioridad de conservación dados sus niveles de amenaza y de uso. 


\begin{abstract}
Cacti are a group of special interest for conservation, because they are threatened mainly by the loss and degradation of habitat and illegal collection, and potentially by climate change. Species of this family establish various mutualistic interactions, like pollination, seed dispersal and establishment of new individuals. Particularly, pollination has been indicated as an essential process on the life cycle of these plants, because many of them depend on this mutualism for their reproduction. In this thesis, the reproductive biology of eight species of the tribe Trichocereeae was studied, with the purpose of knowing in which degree cacti species of the tribe Trichocereeae of northwestern Argentina depend on their pollinators for fruit and seed production, evaluating the potential effects of climate change on the spatial overlap between 12 species of this group of plants and their mutualists, and generating a conservation plan for this group of species and their mutualists.
\end{abstract} Results indicated that eight of the species studied in the first part of the analysis (Cleistocactus baumannii, C. smaragdiflorus, Denmoza rhodacantha, Echinopsis albispinosa, E. haematantha, E. leucantha, E. thionantha and Gymnocalycium saglionis) are self-incompatible, and therefore, they depend on their pollinators to set fruits and seeds. Among the pollinators of these species, bees, moths, wasps and birds were registered, and they showed generalized pollination systems for most cases, at least functionally. The available information for the whole family indicated that phenotypical specialization to birds is a common feature in the cactus family; however, this is not predictive of specialization at a functional level, because for almost every cacti species pollinated by birds, other groups of pollinators were registered, including bees, bats, moths and others. According to the results, the first case of specialized pollination in all modes (phenotypically, functionally and ecologically) was registered for this family: the Denmoza rhodacantha-Oreotrochilus leucopleurus system. The effects of climate change on cacti species studied here were positive for some species (expansion of the geographic range in E. atacamensis, E. leucantha y G. saglionis) and negative for others (contraction of the geographic range in $C$. baumannii, C. smaragdiflorus, $E$. albispinosa, E. ancistrophora, E. haematantha, E. leucantha, E. schickendantzii, E. terscheckii and E. thelegona). The obtained results highlight the importance of 
incorporating biotic interactions to the species geographic range, because they can limit to a large degree the areas that species occupy; however, for most cases, climate change would not cause a decrease in the spatial overlap between cacti and their pollinators, with the exception of the species that showed a specialized pollination system ( $D$. rhodacantha). For this species, spatial mismatch would increase under future climate change scenarios. Priority areas for conservation selected considering the overlapped geographic range had a very low congruence level (26\% of shared areas) with regard to the areas selected without considering the pollinators. Similarly, the priority areas selected under climate change scenarios had a low congruence level (between 12 and $15 \%$, depending on the used scenario) with regard to the areas selected for the present. Based on these results, priority areas that were common to all scenarios, both present and future, were proposed. The latter were a total of five areas, distributed in Salta and La Rioja provinces. These areas are important to generate long-term conservation strategies for cacti and their pollinators. Based on the results of this thesis, future studies should incorporate the other positive interactions that cacti establish with seed dispersers and nurse plants for the evaluation of threats impacts and the definition of in situ conservation strategies. The approach proposed in this thesis could also be used for similar studies on other plant species that have conservation priorities because of their threats and use levels. 


\section{INTRODUCCIÓN GENERAL}

Uno de los efectos más importantes de las actividades humanas a escala mundial es la pérdida de biodiversidad debido principalmente al cambio en el uso del suelo. Como consecuencia, los rangos geográficos de las especies se han restringido y las poblaciones de muchas de ellas han declinado en número; inclusive, algunas especies se han extinguido globalmente y otras lo han hecho a escala local (Ceballos y Erlich 2002, 2006, Parmesan 2006, Bellard et al. 2012). Además de estos efectos, la expansión y crecimiento de la población humana ha significado otros impactos sobre la diversidad biológica como lo son la introducción de especies exóticas, la sobreexplotación, la contaminación de ambientes acuáticos y terrestres y el cambio climático (Sala et al. 2000, Primack 2008, Sodhi 2010). Por

lo tanto, se requieren estrategias que permitan la conservación de la biodiversidad a largo plazo. La importancia de generar acciones concretas de conservación radica en el hecho de que la biodiversidad brinda numerosos servicios ambientales a la humanidad de los que nuestra especie es dependiente. Por ejemplo, el proceso de polinización brinda un servicio ecológico y económico que permite sustentar la vida sobre la tierra, inclusive de la humanidad (Kearns et al. 1998, Kremen et al. 2007). A pesar de la importancia que tienen los procesos que contemplan interacciones entre especies en brindar servicios ambientales, la mayoría de las estrategias de conservación se han concentrado en una especie focal o en grupos de especies emparentadas, sin tener en cuenta a las especies con las cuales éstas interactúan.

La polinización es un proceso fundamental para la reproducción sexual de las plantas. Se estima que el $90 \%$ de las plantas con flores, entre las que se incluyen numerosas especies cultivadas de las cuales depende la alimentación humana (Kearns et al. 1998), es polinizado por animales. Este proceso limita la producción de frutos y semillas en mayor o menor medida dependiendo de las características del sistema reproductivo de las especies; por lo tanto, tiene vital importancia en la dinámica poblacional de las especies de plantas (Stebbins 1970 , Primack 1987). De igual manera, la dinámica poblacional de los polinizadores depende de los recursos provistos por las plantas con las que establecen esta relación mutualista (Kearns et al. 1998). 
Los sistemas de polinización pueden enfrentar amenazas cuando se pierde o se altera alguno de sus componentes, provocando un desacople o disrupción en esta interacción. La disrupción de los sistemas de polinización ha sido registrada en numerosos ambientes alrededor del mundo en las últimas décadas, lo que ha llevado a plantear la idea de que existe una crisis global en el proceso de polinización (Kearns et al. 1998, Potts et al. 2010). Esta crisis sería consecuencia de factores como la fragmentación del hábitat, la expansión de la agricultura y la ganadería, el uso de pesticidas y herbicidas, la introducción de especies exóticas y el cambio climático (Kearns et al. 1998, Traveset y Richardson 2006, Aguilar et al. 2006, Memmott et al. 2007, Hegland et al. 2009, Potts et al. 2010, Schweiger et al. 2010). Los efectos de esta crisis sobre las especies interactuantes pueden variar en su magnitud, dependiendo de sus características biológicas y de las variables ambientales. Por ejemplo, las especies de plantas autoincompatibles muestran un menor éxito reproductivo que las especies autocompatibles en ambientes con niveles mayores de perturbación (Aguilar et al. 2006). Si bien existe un incremento en el número de estudios sobre la disrupción de los sistemas de polinización, los estudios sobre el impacto del cambio climático son aún incipientes (HilleRisLambers et al. 2013). El cambio climático tiene el potencial de provocar disrupción en las interacciones tróficas. Particularmente, los efectos de este fenómeno sobre la interacción planta-polinizador serían la disrupción temporal y espacial entre las plantas y sus polinizadores. La disrupción temporal se expresará en un desacople entre la fenología floral y el período de actividad de los polinizadores, mientras que la disrupción espacial sería consecuencia de cambios en la distribución geográfica entre las especies interactuantes (Parmesan 2006, Memmott et al. 2007, Hegland et al. 2009, Schweiger et al. 2010, 2012).

Las cactáceas son un grupo de especial interés para conservación ya que están amenazadas, principalmente por la pérdida y degradación del hábitat y la colecta ilegal y potencialmente por el cambio climático (Ortega-Baes et al. 2010, Goettsch et al. 2015). Las cactáceas están sometidas a una gran presión de colecta debido a su valor de uso, ya sea como plantas ornamentales, medicinales, comestibles o como fuente de madera. En este sentido, algunas especies de cactáceas están incluidas en el Apéndice I de CITES y toda la familia en el 
Apéndice II. En la actualidad, más del $30 \%$ del total de especies de esta familia están categorizadas por la Unión Internacional para la Conservación de la Naturaleza (IUCN), al menos como amenazadas (Goettsch et al. 2015). Por ello, deben hacerse esfuerzos para obtener información de base acerca de las especies e instrumentar estrategias de conservación para este grupo de plantas (Ortega-Baes et al. 2010, Goettsch et al. 2015).

En las regiones áridas y semiáridas donde se distribuyen, las cactáceas establecen numerosas interacciones biológicas con otras especies de plantas y animales, asociadas a la polinización, la dispersión de semillas o al establecimiento de nuevos individuos (Pimienta-Barrios y del Castillo 2002, Godínez-Álvarez et al. 2003). Este grupo de plantas presenta una gran diversidad en su morfología floral que ha sido asociada a la polinización por animales como insectos, aves y mamíferos. Se ha indicado que los sistemas de polinización que presentan este grupo de plantas serían especializados con base en las características de las flores (Pimienta-Barrios y del Castillo 2002). Sin embargo, la mayoría de los sistemas estudiados hasta la actualidad son generalistas, aun cuando las características de las flores sugieren especialización a polinizadores específicos (Fleming et al. 2001, Ortega-Baes et al. 2011, Alonso-Pedano y Ortega-Baes 2012, Ortega-Baes y Gorostiague 2013). Por ejemplo, las cactáceas columnares con flores nocturnas del hemisferio norte muestran diferentes grados de dependencia hacia los murciélagos nectaríferos, presentando muchos de ellos sistemas generalistas, en los que aves e insectos diurnos son también polinizadores efectivos (Fleming et al. 2001, Munguía-Rosas et al. 2009, Bustamante et al. 2010).

En Argentina, las cactáceas están representadas por más de 200 especies, destacándose Salta como la provincia más diversa (Ortega-Baes et al. 2015). Si bien los estudios sobre la biología del grupo han incrementado en los últimos años (Godínez-Álvarez et al .2003, Flores et al. 2006, Bárcenas et al. 2011), existe una necesidad de profundizar sobre aspectos de su biología, así como extenderlos a especies de muchos linajes que no han sido estudiados todavía. Esta información de base permitirá generar esquemas de conservación para las cactáceas que 
incluyan a aquellas especies de otros grupos con las que establecen interacciones mutualistas y que resultan importantes en los procesos de su ciclo de vida.

Bajo este marco teórico, el propósito general de esta tesis fue estudiar el grado de dependencia de los polinizadores que tienen las cactáceas de la tribu Trichocereeae para la producción de frutos y semillas, evaluando los potenciales efectos del cambio climático sobre el acople espacial entre las especies de este grupo de plantas y sus mutualistas, generando un esquema de conservación para este grupo de especies y sus polinizadores. Particularmente, se utilizó como modelo de estudio a las especies de esta tribu que se distribuyen en el noroeste de Argentina, la región más austral del segundo centro de diversidad a escala global para este grupo de plantas (Ortega-Baes et al. 2010). Las especies para las que se estudiaron aspectos de su biología reproductiva en el presente trabajo fueron Cleistocactus baumannii (Lem.) Lem., Cleistocactus smaragdiflorus (F.A.C. Weber) Britton \& Rose, Denmoza rhodacantha (Salm-Dyck) Britton \& Rose, Echinopsis albispinosa K. Schum., Echinopsis haematantha (Speg.) D.R. Hunt, Echinopsis leucantha Schum., Echinopsis thionantha (Speg.) Werderm. y Gymnocalycium saglionis (F. Cels) Britton \& Rose. Adicionalmente, las especies de las cuales se utilizó información de trabajos publicados previamente para algunos análisis en este trabajo fueron Echinopsis ancistrophora Speg., Echinopsis atacamensis (Phil.) H. Friedrich \& G.D. Rowley, Echinopsis schickendantzii F.A.C. Weber, Echinopsis terscheckii (J. Parm. ex Pfeiff.) H. Friedrich \& G.D. Rowley y Echinopsis thelegona (F.A.C. Weber) H. Friedrich \& G.D. Rowley.

En el Capítulo 1, titulado Biología reproductiva de las cactáceas de la tribu Trichocereeae en el Noroeste de Argentina, se pusieron a prueba las siguientes hipótesis: 1) las cactáceas de la tribu Trichocereeae del noroeste de Argentina dependen de sus polinizadores para la producción de frutos y semillas y 2) los sistemas de polinización en las especies de este grupo son generalistas, a pesar de lo que indican los rasgos florales.

Bajo el título ¿Qué tan especializados son los sistemas de polinización en la familia Cactaceae?: el caso de la polinización por aves, en el Capítulo 2 
se probó la idea de que las cactáceas con flores ornitófilas establecen relaciones especializadas (tanto fenotípica como funcionalmente) con las aves.

En el Capítulo 3, titulado Efecto del cambio climático sobre la distribución geográfica de 12 especies de cactáceas del Noroeste de Argentina y el acople espacial con sus polinizadores, se pusieron a prueba las siguientes ideas: 1) debido a que el aumento en las condiciones de aridez en el continente americano favoreció la radiación de las cactáceas en el pasado, el efecto del cambio climático, asociado a un incremento en las condiciones de aridez, se expresará en un incremento del rango geográfico de las especies de este grupo de plantas y 2) el cambio climático generará un desacople espacial entre las cactáceas y sus polinizadores.

Finalmente, el Capítulo 4 se titula Estrategias de conservación in situ para 12 especies de cactáceas del Noroeste de Argentina. En el mismo, se pusieron a prueba las siguientes hipótesis: 1) si el nivel de acople espacial entre las cactáceas y sus polinizadores es alto, habrá un alto nivel de congruencia entre las áreas prioritarias para la conservación seleccionadas a partir de la distribución de las cactáceas y las seleccionadas a partir de la distribución que comparten (acople espacial) con sus polinizadores y 2) las áreas prioritarias para la conservación de este grupo de especies tendrán un alto nivel de congruencia con las áreas que ocuparán en el futuro bajo escenarios de cambio climático.

\section{REFERENCIAS BIBLIOGRÁFICAS}

Aguilar R., Ashworth L., Galetto R., Aizen M.A. (2006) Plant reproductive susceptibility to habitat fragmentation: review and synthesis through a metaanalysis. Ecology Letters 9: 968-980.

Alonso-Pedano M., Ortega-Baes P. (2012) Generalized and complementary pollination system in the Andean cactus Echinopsis schickendantzii. Plant Systematics and Evolution 298: 1671-1677.

Bárcenas R.T., Yesson C., Hawkins J.A. (2011) Molecular systematics of the Cactaceae. Cladistics 27: 470-489.

Barnosky A.D., Matzke N., Tomiya S., Wogan G.O., Swartz B., Quental T.B., Marshall C., McGuire J.L., Lindsey E.L., Maguire K.C., Mersey B., Ferrer E.A. (2011). Has the Earth's sixth mass extinction already arrived? Nature 
471: 51-57.

Bellard C., Bertelsmeier C., Leadley P., Thuiller W., Courchamp F. (2012) Impacts of climate change on the future of biodiversity. Ecology letters 15: 365-377.

Bustamante E., Casas A., Búrquez A. (2010) Geographic variation in reproductive success of Stenocereus thurberi (Cactaceae): Effects of pollination timing and pollinator guild. American Jounal of Botany 97: 2020-2030.

Ceballos G., Ehrlich P.R. (2002). Mammal population losses and the extinction crisis. Science 296: 904-907.

Ceballos G., Ehrlich P.R. (2006) Global mammal distributions, biodiversity hotspots, and conservation. Proceedings of the National Academy of Sciences 103: 19374-19379.

Fleming T.H., Shaley C.T., Holland J.N., Nason J.D., Hamrick J.L. (2001) Sonoran desert columnar cacti and the evolution of generalized pollination systems. Ecological Monographs 71: 511-530.

Flores J., Jurado E., Arredondo A. (2006) Effect of light on germination of seeds of Cactaceae from the Chihuahuan Desert, México. Seed Science Research 16: 149-155.

Godínez-Alvarez H.O., Valverde T., Ortega-Baes P. (2003) Demographic trends in the Cactaceae. Botanical Review 69: 173-203.

Goettsch B., Hilton-Taylor C., Cruz-Piñón G., Duffy J.P., Frances A., Hernández H.M., et al. (2015). High proportion of cactus species threatened with extinction. Nature plants 1: 15142.

Hegland S.J., Nielsen A., Lázaro A., Bjerknes A.L., Totland Ø. (2009) How does climate warming affect plant-pollinator interactions? Ecology Letters 12: 184195.

HilleRisLambers J., Harsch M.A., Ettinger A.K., Ford K.R., Theobald E.J. (2013). How will biotic interactions influence climate change-induced range shifts? Annals of the New York Academy of Sciences 1297: 112-125.

Kearns C., Inouye D., Waser N. (1998) Endangered mutualisms: the conservation of plant-pollinator interactions. Annual Review of Ecology and Systematics 29: 83-112.

Kremen C., Williams N.M., Aizen M.A., Gemill-Herren B., Lebuhn G., Minckley R., 
Packer L., Potts S.G., Roulston T., Steffan-Dewenter I., Vázquez D.P., Winfree R., Adams L., Crone E.E., Greenleaf S.S., Keitt T.H., Klein A.M., Regetz J., Ricketts T.H.. (2007) Pollination and other ecosystem services produced by mobile orgaisms: a conceptual framework for the effects of land use change. Ecology Letters10: 299-314.

Memmott J., Craze P.G., Waser N.M., Price M.V. (2007) Global warming and the disruption of plant-pollinator interactions. Ecology Letters 10: 710-717.

Munguía-Rosas M.A., Sosa V.J., Ojeda M.M., De-Nova J.A. (2009) Specialization clines in the pollination systems of agaves (Agavaceae) and columnar cacti (Cactaceae): a phylogenetically controlled meta-analysis. American Journal of Botany 96: 1887-1895.

Ortega-Baes P., Shüring S., Sajama J., Sotola E., Alonso Pedano M., Bravo S., Godínez-Alvarez H. (2010) Diversity and conservation in the cactus family. En: Ramawat K. (ed). Desert Plants. Biology and Biotechnology. Springer.

Ortega-Baes P., Saravia M., Sühring S., Godínez-Alvarez H., Zamar M.I. (2011) Reproductive biology of Echinopsis terscheckii (Cactaceae): the role of nocturnal and diurnal pollinators. Plant Biology 13: 33-40.

Ortega-Baes P., Gorostiague P. (2013) Extremely reduced sexual reproduction in the clonal cactus Echinopsis thelegona. Plant Systematics and Evolution 299: 785-791.

Parmesan C. (2006). Ecological and evolutionary responses to recent climate change. Annual Review of Ecology, Evolution, and Systematics 37: 637669.

Pimienta-Barrios E., del Castillo R.F. (2002) Reproductive biology. En: Nobel P.S. (ed) Cacti: biology and uses. University of California Press. pp. 163-183. Potts S.G., Biesmeijer J.C., Kremen C., Neumann P., Schweiger O., Kunin W.E. (2010) Global pollinator declines: trends, impacts and drivers. Trends in ecology \& evolution 25: 345-353.

Primack R.B. (1987) Relationships among flowers, fruits, and seeds. Annual Review of Ecology and Systematics 18: 409-430.

Primack R.B. (2008) A primer of Conservation Biology. Sinauer. Sunderland, USA. Sala O.E., Stuart C.F., Armesto J.J., Berlow E., Bloomfield J., Dirzo R., Huber- 
Sanwald E., Huenneke L.F., Jackson R.B., Kinzig A., Leemans R., Lodge D.M., Mooney H.A., Oesterheld M., LeRoy Poff N., Sykes M.T., Walker B.H., Walker M., Wall D.H. (2000) Global Biodiversity Scenarios for the year 2100. Science 287: 1770-1774.

Schweiger O., Biesmeijer J.C., Bommarco R., Hickler T., Hulme P.E., Klotz S., Kühn I., Moora M., Nielsen A., Ohlemüller R., Petanidou T., Potts S.G., Pysek P., Stout J.C., Sykes M.T., Tscheulin T., Vilà M., Walther G., Westphal C., Winter M., Zobel M., Settele J. (2010) Multiple stressors on biotic interactions: how climate change and alien species interact to affect pollination. Biological Reviews 85: 777-795.

Schweiger O., Heikkinen R.K., Harpke A., Hickler T., Klotz S., Kudrna O., Kühn I., Pöyry J., Settele J. (2012). Increasing range mismatching of interacting species under global change is related to their ecological characteristics. Global Ecology and Biogeography 21: 88-99.

Sodhi N.S. (2010) Conservation biology for all. Oxford University Press.

Stebbins G.L. (1970) Adaptive radiation of reproductive characteristics in angiosperms I: Pollination mechanisms. Annual Review of Ecology and Systematics 1: 307-26.

Traveset A., Richardson D.M. (2006) Biological invasions as disruptors of plant reproductive mutualisms. Trends in Ecology \& Evolution 21: 208-216. 


\section{CAPÍTULO I}

Biología reproductiva de las cactáceas de la tribu Trichocereeae en el Noroeste de Argentina 


\section{INTRODUCCIÓN}

La mayoría de las plantas con flores establecen interacciones mutualistas con los animales que polinizan sus flores. Para las plantas, estas interacciones son de vital importancia para la reproducción, sobre todo en especies auto-incompatibles que necesitan obligadamente a los vectores de polen para la producción de frutos y semillas. Por otro lado, muchos animales mantienen sus poblaciones gracias a los recursos ofrecidos por las flores, ya sea néctar, polen u otros recursos. La gran diversidad floral observada en las angiospermas ha sido asociada a la presión selectiva que ejercen los animales que polinizan sus flores, dada la dependencia de las plantas de esta interacción y sus efectos a nivel individual y poblacional (Stebbins 1970). Tradicionalmente, se ha considerado que las flores presentan adaptaciones especializadas a un gremio particular de polinizadores, y es posible clasificar a las especies con base en estas adaptaciones. Este concepto, llamado síndrome floral, está basado en las características fenotípicas de las flores tales como la forma, el tamaño, el color, el olor, las características del néctar, el ciclo floral, entre otras. (Faegri y van der Pijl 1979). El concepto de síndrome floral ha sido usado para predecir qué animales polinizan una especie de planta (Fenster 2004, Rosas-Guerrero et al. 2014), asumiendo que las especies son especializadas al grupo de polinizadores correspondiente al síndrome floral que muestran sus flores; sin embargo, en muchos casos los animales que visitan las flores no se corresponden con los que se esperaría de acuerdo a las características fenotípicas de las flores (Waser et al. 1996, Ollerton et al. 2009).

En las últimas décadas, muchos estudios demostraron que la mayoría de los sistemas de polinización no son especializados sino generalistas (Waser et al. 1996, Ollerton et al. 2009). Por lo tanto, el concepto de síndrome floral no sería tan eficiente en predecir a todos los animales que polinizan las flores. Sin embargo, un estudio reciente ha demostrado que el síndrome puede predecir al grupo de polinizadores más efectivos (Rosas-Guerrero et al. 2014, Ashworth et al. 2015). Ollerton et al. (2007) proponen que para superar este conflicto se deben considerar diferentes modos en los que las plantas pueden ser generalistas 0 especialistas: la especialización fenotípica (asociada a las características propias de las flores, es decir, los síndromes florales), la especialización funcional (referida 
a los grupos o gremios de animales que visitan las flores de una especie) y la especialización ecológica (referida a las especies de animales que visitan las flores de cierta especie de planta). Al utilizar esta propuesta para clasificar a las plantas según sus sistemas de polinización, es posible evaluar la especialización o generalización teniendo en cuenta no sólo las características propias de las flores, sino también incluyendo la información acerca de los animales con los que establece la interacción. Dado que en numerosos casos los animales que polinizan las flores de una especie no corresponden a lo esperado con base en las características fenotípicas de la planta, es importante identificar cuáles son los polinizadores de los que efectivamente depende la planta para su reproducción (Ollerton 1996, Waser et al. 1996, Hegland y Totland 2005, Ollerton et al. 2007).

Las cactáceas establecen numerosas interacciones mutualistas con otros organismos, asociadas principalmente a la polinización de sus flores, la dispersión de sus semillas y al establecimiento de nuevos individuos (Pimienta-Barrios y del Castillo 2002, Godínez-Álvarez et al. 2003). Particularmente, este grupo de plantas provee un buen modelo para explorar los sistemas de polinización debido a que presentan una gran diversidad floral y también una variedad de animales polinizadores. Tradicionalmente, se ha considerado que las flores de las cactáceas son especializadas a la polinización por ciertos gremios de polinizadores específicos, que incluyen principalmente aves, abejas, murciélagos y mariposas nocturnas (Gibson y Nobel 1986, Pimienta-Barrios y del Castillo 2002). Sin embargo, muchos de los sistemas que fueron estudiados en detalle demostraron ser generalistas, siendo raros los casos documentados de especialización (Fleming et al. 2001, Ortega-Baes et al. 2011). En algunos casos, a pesar de presentar especialización fenotípica en sus flores, los polinizadores registrados como visitantes pertenecen a varios gremios de animales, siendo por lo tanto generalistas desde el punto de vista funcional. Por ejemplo, el cactus columnar norteamericano Carnegiea gigantea presenta flores con un síndrome floral típicamente quiropterófilo; es decir, que de acuerdo a las características de sus flores, se espera que sea polinizado por murciélagos. Sin embargo, estudios realizados sobre las interacciones mutualistas que establece esta especie con sus polinizadores han registrado murciélagos, aves y abejas como polinizadores 
efectivos (Mc Gregor et al. 1962, Fleming et al. 2001,Drezner 2014), así como variación espacial y temporal en la efectividad de dichos polinizadores (Fleming et al. 2001).

Por otro lado, las especies de la familia presentan generalmente un alto nivel de dependencia de los polinizadores, con numerosas especies autoincompatibles (Mandujano et al. 2010). Por lo tanto, es fundamental contar con información acerca de las interacciones planta-polinizador de las que depende la reproducción de estas plantas para planificar acciones de conservación para el grupo (Kearns et al.1998). En la actualidad esta información es limitada, con una proporción baja de especies estudiadas, con un sesgo en los estudios hacia la flora cactológica del hemisferio norte. Por ejemplo, sólo se conoce el sistema reproductivo y los polinizadores para menos del $5 \%$ del total de cactáceas que habitan en el noroeste de Argentina, la región más diversa del país.

La tribu Trichocereeae (subfamilia Cactoideae) se distribuye principalmente en América del Sur y posee su centro de diversidad en los Andes (Ortega-Baes et al. 2015). Las especies de esta tribu presentan diversas formas de vida y también una gran diversidad en sus flores. A pesar de esto, existen al día de la fecha pocos estudios sobre la biología reproductiva de estas especies (Sahley 1996, Schlumpberger y Badano 2005, Schlumpberger et al. 2009, Walter 2010, LarreaAlcázar y López 2011, Ortega-Baes et al. 2011, Alonso Pedano y Ortega-Baes 2012, Ortega-Baes y Gorostiague 2013). La variación en la especialización fenotípica con respecto a la polinización también es amplia entre las especies de este grupo: existen especies con flores consideradas ornitófilas (asociadas a la polinización por aves, por ejemplo en los géneros Cleistocactus y Oreocereus), melitóflas (polinización por abejas, por ejemplo el género Gymnocalycium) y esfingófilas (polinización por mariposas nocturnas, por ejemplo algunas especies de los géneros Echinopsis y Harrisia; Anderson 2001, Hunt 2006). La mayoría de los estudios realizados en especies de esta tribu corresponden a especies del género Echinopsis, que a su vez es el más diverso en cuanto a número de especies. Particularmente para este género, Schlumpberger y Renner (2012) analizaron las relaciones filogenéticas y sugirieron que los cambios o transiciones en los síndromes florales (por ejemplo, de flores melitófilas a flores esfingófilas) se 
presentan como un rasgo común a lo largo de la historia evolutiva del género, así como en otros grupos de angiospermas (van der Niet y Johnson 2012). Esta idea está basada en el supuesto que los rasgos florales implican necesariamente que los sistemas de polinización son especializados a aves, abejas o mariposas nocturnas (Schlumpberger y Renner 2012). Sin embargo, esta suposición podría no ser acertada debido a que no tiene en cuenta al ensamble de polinizadores que realmente interactúa con cada especie de este género. Por ejemplo, los animales nocturnos pueden tener una mayor efectividad como polinizadores y por lo tanto un papel más importante en la producción de frutos y semillas en algunas especies (Ortega-Baes et al. 2011), mientras que otras pueden depender de los polinizadores diurnos (Schlumpberger y Badano 2005), más allá de lo que indiquen los rasgos florales. Inclusive los polinizadores diurnos y nocturnos pueden llegar a ser igualmente efectivos o complementarios (Walter 2010, AlonsoPedano y Ortega-Baes 2012). Así, los rasgos florales mostrados por este grupo de especies no necesariamente corresponden con los animales quepolinizan efectivamente sus flores. Estos ejemplos destacan la importancia de realizar estudios comprensivos sobre la biología reproductiva de estas especies, documentando el papel de los visitantes florales en la reproducción de las plantas, más allá de las predicciones basadas en sus rasgos fenotípicos, especialmente en el caso de especies auto-incompatibles que dependen de sus visitantes florales para la producción de frutos y semillas (Rosas-Gerrero et al. 2014). Para comprender de qué manera los sistemas de polinización en la familia Cactaceae han evolucionado hacia la generalización o especialización, es necesario incrementar significativamente el número de estudios sobre estos aspectos, particularmente en regiones donde esta información es escasa o nula, y en linajes poco o nada estudiados al presente.

Bajo este marco teórico, en el presente capítulo se pusieron a prueba las siguientes hipótesis: 1) las cactáceas de la tribu Trichocereeae del noroeste de Argentina dependen de sus polinizadores para la producción de frutos y semillas y 2) los sistemas de polinización en las especies de este grupo son generalistas, a pesar de lo que indican sus rasgos fenotípicos. Para esto, se intentó responder a las siguientes preguntas: a) ¿presentan las flores de las especies de la tribu 
Trichocereeae del noroeste de Argentina especialización fenotípica?; b) ¿cuál es el tiempo de antesis y la duración de la misma en las especies bajo estudio?; c) ¿en qué grado dependen las especies bajo estudio de sus polinizadores para la producción de frutos y semillas?; d) ¿cuáles son los animales que polinizan las flores de las especies bajo estudio y qué tan efectivos son?; y e) ¿qué tan especializados son los sistemas de polinización en las especies de la tribu Trichocereeae del noroeste de Argentina?.

\section{MATERIALES Y MÉTODOS}

\section{Especies bajo estudio}

La tribu Trichocereeae pertenece a la subfamilia Cactoideae, la más diversa de la familia Cactaceae. Dentro de esta tribu se incluyen especies con forma de crecimiento globosa, columnar corta, columnar arborescente y columnar rastrera. Las especies se distribuyen a lo largo de América del Sur y su centro de diversidad se concentra en los Andes (Hunt 2006). Esta tribu está representada en el noroeste de Argentina por especies de los géneros Cleistocactus, Denmoza, Echinopsis, Gymnocalycium, Harrisia, Oreocereus y Rebutia (Hunt 2006). Se estudió la biología reproductiva de ocho especies de cuatro de estos siete géneros: Cleistocactus baumannii (Fig. 1), Cleistocactus smaragdiflorus (Fig. 2), Denmoza rhodacantha (Fig. 3), Echinopsis albispinosa (Fig. 4), Echinopsis haematantha (Fig. 5), Echinopsis leucantha (Fig. 6), Echinopsis thionantha (Fig. 7) y Gymnocalycium saglionis (Fig. 8). Además, en este estudio, se incluyeron en el análisis especies de la tribu con las que ya se contaba información sobre la biología reproductiva: Echinopsis ancistrophora (Schlumpberger y Raguso 2008, Schlumperger et al. 2009), Echinopsis atacamensis (de Viana et al. 2001, Schlumpberger y Badano 2005), Echinopsis schickendantzii (Alonso-Pedano y Ortega-Baes 2012), Echinopsis terscheckii (Ortega-Baes et al. 2011) y Echinopsis thelegona (Ortega-Baes y Gorostiague 2013).

\section{Área de estudio}

El estudio se llevó a cabo en la provincia de Salta, donde se seleccionó una población para cada una de las especies mencionadas. Las especies se distribuyeron en la ecorregión del Monte Alto (D. rhodacantha, E. haematantha, $E$. 
leucantha y E. thionantha) y en la ecorregión del Chaco Seco (C. baumannii, $C$. smaragdiflorus, E. albispinosa y G. saglionis; Olson y Dirnerstein 2002; Fig. 9).

\section{Características fenotípicas y ciclo floral}

A fin de evaluar si las flores de las especies bajo estudio presentan especialización fenotípica a tipos de polinizadores específicos se colectaron 20 flores abiertas de un mínimo de diez individuos seleccionados al azar. Para cada flor se registró su largo total, su color, el diámetro de la corola y se clasificó la forma de la flor. De acuerdo a la literatura (Hunt 2006), se consideraron las siguientes formas florales: 1) tubular, 2) tazón y 3) embudo. De acuerdo a las características fenotípicas de las flores, las especies fueron clasificadas en los diferente síndromes florales descriptos para las cactáceas (Pimienta Barrios y del Castillo 2002, Mandujano et al. 2010).

Para conocer el tiempo y duración de la antesis floral, se seleccionaron al azar y se marcaron 20 botones florales que fueron cubiertos con bolsas de voile para aislarlos de la actividad de los visitantes florales. Se registró, cada dos horas, el estadío fenológico de cada botón, desde la apertura hasta la senescencia de las flores. Los estadíos considerados fueron: botón floral, flor parcialmente abierta, flor completamente abierta, flor parcialmente cerrada, y flor completamente cerrada. También se registró si las flores permanecían abiertas durante la noche, y en el caso que cerraran, si las mismas abrían al día siguiente.

\section{Sistema reproductivo}

A fin de conocer el sistema reproductivo, se realizó un experimento de campo para cada una de las ocho especies bajo estudio. Los tratamientos evaluados fueron los siguientes: 1) polinización natural: flores sin manipular; 2) auto-polinización automática: flores excluidas sin manipulación; 3) auto-polinización manual: flores emasculadas polinizadas manualmente usando polen de la misma flor y polen de otras flores del mismo individuo y 4) polinización cruzada manual: flores emasculadas polinizadas manualmente con polen de flores de otros individuos. Las flores de los tratamientos 2), 3) y 4) fueron asiladas de los posibles polinizadores cubriéndolas con bolsas de voile luego de ser tratadas. El número de flores utilizadas en cada tratamiento varió en función de la disponibilidad presente en la población, aunque el mínimo fue de 15 flores por tratamiento Las variables 
de respuesta fueron la relación frutos/flores y la relación semillas/óvulos, esta última registrada en todos los frutos formados. Los valores de la relación frutos/flores entre tratamientos de sistema reproductivo fueron comparados usando una prueba exacta de Fisher, mientras que los valores de la relación semillas/óvulos fueron comparados con una prueba t. Los análisis estadísticos se realizaron utilizando el software Infostat versión 2009 (Di Rienzo et al. 2009).

\section{Visitantes florales $y$ su efectividad}

Para conocer los visitantes florales se realizaron observaciones focales sobre tres a cinco flores (dependiendo del número de flores disponibles) por población a lo largo del ciclo floral (durante 30 minutos cada tres horas). Estas observaciones se realizaron en al menos tres momentos a lo largo de la estación de floración. Se consideró como polinizador efectivo sólo a aquel que tocó las estructuras reproductivas durante la visita. Los insectos visitantes fueron colectados con redes de mano y llevados al laboratorio para su acondicionamiento y posterior envío al Museo Argentino de Ciencias Naturales Bernardino Rivadavia (Buenos Aires) para su determinación. Las aves se identificaron mediante una guía de campo (Narosky y Yzurrieta 2010). Las observaciones nocturnas se realizaron con luz tenue y, además, se colectaron estigmas de las flores para registrar la presencia de escamas de mariposas nocturnas. La presencia de escamas de mariposas nocturnas fue considerada como evidencia de que la flor fue visitada por estos animales. Adicionalmente, en cada sitio de estudio, se montó una trampa de luz UV durante tres noches (desde las 20:00 hs hasta las 01:00 hs del día siguiente) para colectar mariposas nocturnas. Las mariposas nocturnas capturadas fueron acondicionadas, independientemente, en camas de algodón y llevadas al laboratorio para su identificación con base en una colección de referencia. En cada ejemplar capturado, se frotaron sobre la cabeza y el cuerpo cubos de gelatina glicerinada (de $3 \times 3 \mathrm{~mm}$ de tamaño aproximado), con los que se realizaron preparados semipermanentes para la identificación al microscopio óptico de granos de polen de cada especie. Para esto se utilizó una colección de referencia de preparados de granos de polen de las especies bajo estudio.

En los sistemas de polinización que presentaron tanto polinizadores nocturnos como diurnos se determinó la efectividad de cada grupo a través de 
experimentos de exclusión. Cada experimento de efectividad consistió en tres tratamientos que incluyeron: polinización natural (flores sin manipulación), polinización nocturna (flores que permanecieron disponibles para los visitantes florales durante la noche y se excluyeron con bolsas de voile antes del amanecer), y polinización diurna (flores que fueron excluidas con bolsas de voile durante la noche y se retiraron al amanecer). El número mínimo de flores utilizadas en cada tratamiento fue de 15, y la variable de respuesta fue la relación flores/frutos. Las diferencias entre tratamientos fueron analizadas utilizando una prueba de Chi cuadrado, y cuando resultó significativa se realizaron particiones del Chi cuadrado (Agresti 2002) para evaluar si existían diferencias entre ambos grupos de polinizadores.

\section{RESULTADOS}

\section{Características fenotípicas y ciclo floral}

De acuerdo a las características de las flores, las especies estudiadas se correspondieron con tres grupos diferentes asociados a especialización fenotípica: las flores melitófilas (o flores de abejas) se registraron en Echinopsis haematantha, E. thionantha y Gymnocalycium saglionis, las cuales presentaron una corola corta con forma de tazón y un diámetro de corola mayor que el largo total, con colores blanco, amarillo o naranja. Las flores ornitófilas (o flores de aves) fueron reconocidas en Cleistocactus baumannii, C. smaragdiflorus y Denmoza rhodacantha. Estas tres especies presentaron flores con forma tubular, con una largo total varias veces mayor al diámetro de la corola y con colores rojo o magenta. Por otro lado, las dos especies restantes (E. albispinosa y E. leucantha), presentaron flores esfingófilas, asociadas a la polinización por mariposas nocturnas. En estas flores el largo total fue mayor que el diámetro de la corola, con forma de embudo y con colores blanco o blanco-rosado. Estas rasgos florales también fueron característicos de las cinco especies previamente estudiadas ( $E$. ancistrophora, E. atacamensis, E. schickendantzii, E. terscheckii y E. thelegona; Tabla 1).

Se registraron tres patrones con relación a la apertura de las flores. Entre las especies con flores melitófilas (Echinopsis haematantha, E. thionantha y Gymnocalycium saglionis), las flores abrieron a la mañana. En las especies con 
flores ornitófilas (Cleistocactus baumannii, C. smaragdiflorus y Denmoza rhodacantha) las flores abrieron a la tarde, mientras que en las especies con flores esfingófilas (E. albispinosa y E. leucantha), la apertura fue al atardecer (Tabla 1). En las especies melitofilas, la antesis fue exclusivamente diurna, cerrando a la tarde. En dos de las especies (E. haematantha y E. thionantha), la longevidad floral fue de dos días, mientras que en $G$. saglionis fue de tres días. Es decir, las flores abren a la mañana, cierran a la tarde y vuelven a abrir a la mañana siguiente. Las flores de las tres especies ornifófilas tuvieron una longevidad de dos días, permaneciendo abiertas durante todo ese período de tiempo. Las flores esfingófilas de las dos especies estudiadas estuvieron abiertas durante la noche y durante la mañana del día siguiente. En estas últimas especies el ciclo floral duró menos de 24 hs. Este mismo comportamiento fue registrado para las especies con flores esfingófilas estudiadas previamente ( $E$. ancistrophora, $E$. atacamensis, $E$. schickendantzii, E. terscheckii y E. thelegona; Tabla 1).

\section{Sistema reproductivo}

Para ninguna de las especies estudiadas, se produjeron frutos en los tratamientos de autopolinización automática y autopolinización manual (Fig. 10). El valor de la relación frutos/flores fue significativamente mayor para el tratamiento de polinización cruzada manual que para la polinización natural en las especies $C$. baumannii $(\mathrm{F}=-0.7, \mathrm{p}<0.0001)$, C. smaragdiflorus $(\mathrm{F}=-0.7, \mathrm{p}<0.0001), E$. albispinosa $(F=-0.67, p<0.0001)$ y $E$. haematantha $(F=26.44, p<0.0001)$. Mientras que no se registraron diferencias significativas entre ambos tratamientos para las especies $D$. rhodacantha $(F=0.27, p=0.1689)$, E. leucantha $(F=0.14, p$ $=0.3869)$, E. thionantha $(F=-0.20, p=0.4139)$ y $G$. saglionis $(F=-0.16,=$ $0.4649)$.

La relación semillas/óvulos de los frutos producidos en los tratamientos de polinización natural y de polinización cruzada manual (Fig. 11) no fue significativamente diferente para ninguna de las especies (C. baumannii: $t=-1.7, p$ $=0.1 ; C$. smaragdiflorus: $\mathrm{t}=0.6, \mathrm{p}=0.7 ; D$. rhodacantha: $\mathrm{t}=1.34, \mathrm{p}=0.2527 ; \mathrm{G}$. saglionis: $\mathrm{t}=0.95, \mathrm{p}=0.3573$ ). Para la especie $E$. leucantha se evaluó la diferencia en la relación semillas/óvulos entre ambos tratamientos utilizando una prueba de Chi cuadrado debido al bajo número de frutos producidos. Esta prueba 
indicó que la relación semillas/óvulos fue mayor para el tratamiento de polinización cruzada manual que para el de polinización natural $\left(X^{2}=1013.21, \mathrm{p}<0.0001, \mathrm{gl}=\right.$ 1). Para las especies $E$. albispinosa, $E$. haematantha y $E$. thionantha no fue posible calcular la relación semillas/óvulos debido a que los frutos maduros producidos se perdieron posiblemente por el ramoneo del ganado caprino.

\section{Visitantes florales $y$ su efectividad}

Los visitantes florales de las especies estudiadas incluyeron abejas, avispas, aves y mariposas nocturnas (Tabla 2). Las especies con flores melitófilas ( $E$. haematantha, E. thionantha y $G$. saglionis) fueron visitadas por abejas y avispas. Las flores ornitófilas de las especies del género Cleistocactus y de Denmoza rhodacantha fueron visitadas por picaflores, siendo C. smaragdiflorus además visitada por abejas del género Xylocopa. Es importante destacar que $D$. rhodacantha fue visitada solamente por el picaflor andino Oreotrochilus leucopleurus. Aun cuando en estas especies las flores estuvieron disponibles durante la noche, no se registraron escamas de mariposas nocturnas en los estigmas. En las flores esfingófilas de $E$. albispinosa y $E$. leucantha sólo se registraron visitas de animales diurnos. En E. albispinosa sólo se registró como visitante floral a la abeja exótica Apis mellifera, mientras que en $E$. leucantha se registró además de esta especie de abeja, al paseriforme Phrygilus gayi (Tabla 2). Aun cuando no se registraron visitas de mariposas nocturnas a las flores de estas especies, susestigmas presentaron escamas de estos insectos. En E. albispinosa, el $54.6 \%$ de los estigmas presentaron escamas de mariposas nocturnas, mientras que en E. leucanthaeste valor fue del $20.5 \%$. En las trampas de luz UV no se capturaron mariposas nocturnas en ninguna de las poblaciones bajo estudio.

Debido a que se registró visitas de animales diurnos y evidencia de visita de animales nocturno (presencia de escamas en el estigma) en $E$. albispinosa y $E$. leucantha se evaluó la efectividad diferencial de esos dos grupos de visitantes florales. Para E. albispinosa, se registraron diferencias significativas en la producción de frutos entre los tratamientos $\left(X^{2}=8.93, p=0.0115, \mathrm{gl}=2\right)$. En el tratamiento de polinización nocturna no se produjeron frutos. De acuerdo a la partición del Chi cuadrado, la relación frutos/flores registrada en el tratamiento de polinización natural diurna no fue significativamente diferente que la del 
tratamiento de polinización natural $\left(X^{2}=2.21, p=0.1374, g l=1\right.$; Fig. 12). Para $E$. leucantha se registraron diferencias significativas en la proporción de frutos producidos entre los tratamientos $\left(X^{2}=8.89, p=0.0117, g l=2\right)$. No se produjeron frutos en el tratamiento de polinización nocturna, y la efectividad de los polinizadores diurnos no fue significativamente diferente de la polinización natural $\left(X^{2}=0.96, p=0.3264, g l=1\right)$.

\section{DISCUSIÓN}

Las especies de la tribu Trichocereeae que habitan en el noroeste de Argentina presentaron una gran diversidad en cuanto a sus rasgos florales, los cuales tradicionalmente han sido asociados a la polinización por parte de diferentes grupos de animales (o síndrome florales, sensu Feagri y van der Pijl 1979). De acuerdo al tamaño y la forma floral, el color y el tiempo de la antesis, las especies bajo estudio podrían agruparse en especies con especialización fenotípica a las abejas o especies melitófilas (Echinopsis haematantha, E. thionantha y Gymnocalycium saglionis), con especialización a las aves u ornitófilas (Cleistocactus ssp. y Denmoza rhodacantha) y con especialización a las mariposas nocturnas o esfingógfilas (E. albispinosa, $E$. atacamensis, $E$. ancistrophora, E. leucantha, E. schickendantzii, E. terscheckii y E. thelegona). Las especies con flores de abejas presentaron corolas de color blanco, amarillo o naranja, con forma de tazón y un ciclo floral exclusivamente diurno, en el que las flores abren a la mañana y cierran durante la tarde. Por lo tanto, las mismas no se encuentran disponibles para ser visitadas por animales nocturnos. Las especies con flores de aves, con corolas tubulares de color rojo o magenta, presentaron apertura floral durante la tarde y sus flores permanecieron abiertas durante dos días, encontrándose disponibles durante la noche para los polinizadores nocturnos. Tradicionalmente, se ha considerado que las cactáceas presentan flores que duran un día (Fleming et al. 2001, Ortega-Baes et al. 2011), Sin embargo, muy pocos estudios se han enfocado en especies con antesis diurna como las presentadas aquí, que mostraron tener ciclos florales más largos.

Para el grupo de especies con flores de mariposas nocturnas se registraron corolas con forma de embudo y de color principalmente blanco. Si bien el ciclo floral de estas especies es considerado tradicionalmente como nocturno (Hunt 
2006), los resultados indicaron que sus flores abren durante el crepúsculo o a la noche, y permanecen abiertas durante parte del día siguiente. Ninguna de las especies de este último grupo permaneció abierta durante más de 24 horas. Por lo tanto, las especies estudiadas en el presente trabajo ( $E$. albispinosa y $E$. leucantha) y las publicadas en estudios anteriores ( $E$. ancistrophora, $E$. atacamensis, E. schichandantzii, E. terscheckii y E. thelegona), presentaron ciclos florales extendidos hasta la mañana siguiente, encontrándose sus flores disponibles para ser visitadas por animales tanto nocturnos como diurnos (Schlumpberger y Badano 2005, Schlumpberger y Raguso 2008, Ortega-Baes et al. 2011, Alonoso-Pedano y Ortega-Baes 2012, Ortega-Baes y Gorostiague 2013). Se ha propuesto para especies de cactáceas norteamericanas de la tribu Pachycereeae que esto podría constituir una estrategia para hacer frente a la escasez de polinizadores, y sería por lo tanto un mecanismo de reaseguro reproductivo. Si la flor no fue polinizada durante la noche, puede ser visitada por animales diurnos que también se comportan como polinizadores efectivos (Fleming et al. 2001, Ortega-Baes et al 2011, Torres-Díaz et al. 2011).

Todos los estudios realizados hasta la fecha sobre biología reproductiva en las especies de la tribu Trichocereeae del noroeste de Argentina demostraron que las especies son auto-incompatibles; es decir, que dependen de la actividad de los polinizadores para la producción de frutos y semillas, confirmando la primera hipótesis planteada para este trabajo. Esta característica parece ser común entre las cactáceas columnares (Mandujano et al. 2010). Sin embargo, se han documentado especies autocompatibles en la tribu Trichocereeae como es el caso de Weberbauerocereus weberbaueri (Sahley 1996), Echinopsis chiloensis (Walter 2010) y Oreocereus celsianus (Larrea-Alcázar y López 2011). En la mayoría de las especies estudiadas se registraron valores más altos de producción de frutos y/o de semillas para el tratamiento de polinización cruzada manual (reflejado en una mayor relación frutos/flores o semillas/óvulos) que en el tratamiento de polinización natural. Esto sugiere que habría limitación por polen (polinizadores) en la producción de frutos y semillas para este grupo de especies, al menos en las poblaciones estudiadas. En el caso particular de la especie E. leucantha, los valores obtenidos para la relación semillas/óvulos sugieren limitación por polen, 
pero no así para los obtenidos para la relación frutos/flores. Para esta última variable de respuesta no se registraron diferencias en la relación frutos/flores entre el tratamiento de polinización natural y el de polinización cruzada. La producción de frutos fue muy baja para ambos tratamientos, lo cual indicaría una limitación por recursos. Esta limitación probablemente sea consecuencia de las condiciones de extrema aridez presentes en el área donde se localizó la población estudiada de esta especie, en donde la baja disponibilidad de agua limitaría la producción de estructuras reproductivas (Godínez-Álvarez et al. 2003). Futuros estudios deberían realizarse para demostrar la existencia de limitación por polen en los sistemas de polinización de las especies estudiadas y la existencia de limitación por polen y recursos en $E$. leucantha.

Entre los polinizadores de las especies de la tribu Trichocereeae del noroeste de Argentina estudiadas en la presente tesis se encontraron abejas, avispas, mariposas nocturnas y aves. Las especies con flores de abejas, de antesis exclusivamente diurna, fueron visitadas por abejas y avispas, mientras que las especies ornitófilas fueron visitadas por picaflores y abejas. Aun cuando las flores de estas especies permanecieron abiertas durante la noche, no se encontró evidencia directa ni indirecta de la visita de animales nocturnos. Esto datos no coinciden con lo registrado para Oreocereus celsianus en los Andes bolivianos, una especie de la misma tribu que también presenta flores ornitófilas, donde se registraron visitas de mariposas nocturnas (Larrea-Alcázar y López 2011). Por otro lado, todas las especies con flores esfingófilas, de antesis crepuscular-nocturna con ciclos extendidos, fueron visitadas por animales tanto nocturnos (mariposas nocturnas) como diurnos (abejas o aves). Este patrón también fue registrado en especies de género Echinopsis que se distribuyen en el noroeste de Argentina (Ortega-Baes et al. 2011, Alonso-Pedano y Ortega-Baes 2012, Ortega-Baes y Gorostiague 2013) y que se distribuyen en Chile (Walter 2010). Entre las abejas, se encontraron tanto especies nativas como a la especie exótica Apis mellifera. Esta última especie fue el polinizador más frecuentemente registrado para dos especies con flores de antesis nocturna (Echinopsis albispinosa y E. leucantha). Para ambas especies, las mariposas nocturnas tuvieron un papel secundario en su contribución a la producción de frutos y semillas. Esto podría deberse a la baja 
abundancia local de estos animales, registrada para algunas especies de cactáceas del noroeste de Argentina (Ortega-Baes y Gorostiague 2013).

Las especies cuyas flores fueron polinizadas por aves fueron C. baumannii, C. smaragdiflorus, $D$. rhodacantha y $E$. leucantha. Las aves polinizadoras en todas las especies fueron picaflores (Trochillidae), a excepción de E. leucantha, cuyas flores fueron visitadas por un ave paseriforme (Prhygilus gayi, Thraupidae). La polinización por aves ya ha sido registrada en numerosos casos para la familia Cactaceae (Ver Capítulo II); sin embargo, E. leucantha constituye el primer caso de una cactácea polinizada por paseriformes en América del Sur continental. Sólo dos casos de cactáceas polinizadas por aves distintas a picaflores son conocidos en la actualidad: Opuntia spp. en las Islas Galápagos que son polinizadas por pinzones (Grant y Grant 1981) y Carnegiea gigantea en Norteamérica que es polinizada principalmente por palomas y también por moscones, pinzones y otras aves (Mc Gregor et al. 1962, Fleming et al. 2001, Drezner 2014).

Teniendo en cuenta el tamaño, la forma, el color, el tiempo y duración de la antesis, las cactáceas de la tribu Trichocereeae del noroeste de Argentina presentaron especialización fenotípica a gremios particulares de polinizadores, tal como se ha citado en general para la familia (Pimienta Barrios y del Castillo 2002). Se pudieron reconocer especies con flores especializadas fenotípicamente a mariposas nocturnas, abejas y aves. Sin embargo, la especialización a nivel funcional (sensu Ollerton et al. 2007) no fue tan común entre las especies del grupo. Entre las especies con flores melitófilas, E. haematantha y E. thionantha fueron polinizadas exclusivamente por abejas, por lo tanto serían especialistas funcionales, mientras que $G$. saglionis sería una generalista funcional debido a que es polinizada por abejas y avispas. Entre las especies con flores ornitófilas, se registraron dos especies que son visitadas exclusivamente por picaflores ( $C$. baumannii y $D$. rhodacantha), es decir, serían funcionalmente especializadas; y una especie que es visitada por picaflores y por abejas (C. smaragdiflorus), siendo por lo tanto funcionalmente generalista. Todas las especies con flores esfingófilas (un total de siete especies incluidas las estudiadas en este trabajo y las estudiadas previamente), fueron funcionalmente generalistas, siendo visitadas por mariposas nocturnas durante la noche y por abejas, aves o avispas durante el día, aunque en 
algunas de estas especies (por ejemplo, E. albispinosa y E. leucantha) no se registró producción de frutos en los tratamientos correspondientes a los polinizadores nocturnos. Por último, la especialización ecológica (asociada al número de especies de polinizadores con los que interactúa la planta; Ollerton et al. 2007) sólo se registró en Denmoza rhodacantha, cuyas flores fueron visitadas exclusivamente por el picaflor Oreotrochilus leucopleurus (Trochillidae), siendo el primer caso de un sistema de polinización especialista en los tres modos que una flor puede ser especializada según Ollerton et al. (2007). El resto de las especies fueron generalistas desde el punto de vista ecológico. Por lo tanto, la evidencia actual indica que aun cuando las flores presentan especialización fenotípica, la mayoría de las cactáceas del noroeste de Argentina presentan sistemas de polinización generalistas (funcional y ecológicamente), confirmando la segunda hipótesis planteada. Particularmente, todas las especies con flores esfingófilas fueron funcionalmente generalistas, a excepción de $E$. albispinosa para la cual no se demostró que los visitantes nocturnos polinicen efectivamente sus flores. En este grupo, la contribución relativa de los polinizadores diurnos y nocturnos puede ser complementaria (por ejemplo E. schickendantzii; Alonso-Pedano y OrtegaBaes 2012), más importante de los visitantes florales nocturnos (E. terscheckii; Ortega-Baes et al. 2011) o más importante de los visitantes diurnos como fue sugerido para $E$. atacamensis (Schlumpberger y Badano 2005) y demostrado en esta tesis para E. albispinosa y E. leucantha. Esto pone en evidencia que el polinizador principal que se esperaría a partir de los rasgos florales (principio del polinizador más efectivo, Stebbins 1970), podría no ser el que realiza el mayor aporte a la reproducción. Estudios futuros deberían analizar si la importancia relativa de los polinizadores diurnos y nocturnos en las especies con flores esfingófilas varía espacial y temporalmente tal como ha sido demostrado en otras especies de la familia (Fleming et al. 2001, Bustamante et al. 2010, AlonsoPedano y Ortega-Baes 2012, Drezner 2014).

Los resultados presentados aquí resaltan la importancia de los muestreos a campo para estudiar las interacciones planta-polinizador, ya que las relaciones establecidas por las especies podrían no ser las esperadas de acuerdo a los rasgos fenotípicos de sus flores. El alto grado de dependencia de los polinizadores 
por parte de este grupo de plantas resalta la importancia que tiene este mutualismo sobre los ciclos de vida de las especies, y ponen en evidencia la necesidad de profundizar los estudios sobre la biología reproductiva de las especies que pertenecen a esta tribu y a otras para las cuales no existe ningún estudio aún o éstos son muy limitados, como es el caso de las tribus Notocacteeae y Cereeae y de la subfamilia Opuntioideae, sobre todo en regiones que representan centros de diversidad taxonómica para la familia, como es el caso del noroeste del país.

\section{REFERENCIAS BIBLIOGRÁFICAS}

Alonso-Pedano M., Ortega-Baes P. (2012) Generalized and complementary pollination system in the Andean cactus Echinopsis schickendantzii. Plant Systematics and Evolution 298: 1671-1677.

Anderson E.F. (2001) The cactus family. Portland, USA. Timber Press. 776p.

Ashworth L., Aguilar R., Martén-Rodríguez S., Lopezaraiza-Mikel M., Avila-Sakar G., Rosas-Guerrero V., Quesada M. (2015) Pollination syndromes: a global pattern of convergent evolution driven by the most effective pollinator. En: Pontarotti P. (ed.) Evolutionary biology: biodiversification from genotype to phenotype. Springer International Publishing: 203-224.

Bustamante E., Casas A., Búrquez, A. (2010) Geographic variation in reproductive success of Stenocereus thurberi (Cactaceae): effects of pollination timing and pollinator guild. American Journal of Botany 97: 2020-2030.

de Viana M.L., Ortega-Baes P., Saravia M., Badano E., Schlumpberger B. (2001) Biología floral y polinizadores de Trichocereus pasacana (Cactaceae) en el Parque Nacional Los Cardones, Argentina. Revista de Biología Tropical 49: 279-285.

Di Rienzo J.A., Casanoves F., Balzarini M.G., Gonzalez L., Tablada M., Robledo C.W. (2009) InfoStat versión 2009. Argentina: Grupo InfoStat, FCA, Universidad Nacional de Córdoba.

Drezner T.D. (2014) The keystone saguaro (Carnegiea gigantea, Cactaceae): a review of its ecology, associations, reproduction, limits, and demographics. Plant ecology 215(6): 581-595.

Faegri K., van der Pijl L. (1979) The principles of pollination ecology. London: 
Pergamon Press: 248 pp.

Fenster C.B., Armbruster W.S., Dudash M.R., Wilson P., Thomson J.D. (2004)

Pollination syndromes and floral specialization. Annual Review of Ecology and Systematics 35: 375-103.

Fleming T.H., Shaley C.T., Holland J.N., Nason J.D., Hamrick J.L. (2001) Sonoran desert columnar cacti and the evolution of generalized pollination systems. Ecological Monographs 71: 511-530.

Gibson A.C., Nobel P.S. (1986) The cactus primer. Harvard University Press, Cambridge, USA: $286 \mathrm{pp}$.

Godínez-Alvarez H.O., Valverde T., Ortega-Baes P. (2003) Demographic trends in the Cactaceae. Botanical Review 69: 173-203.

Grant B.R., Grant P.R. (1981) Exploitation of Opuntia cactus by Birds on the Galápagos. Oecologia 49: 179-187.

Hegland S.J., Totland O. (2005) Relationships between species floral traits and pollinator visitation in a temperate grassland. Oecologia 145: 586-594.

Hunt D. (2006) The new cactus lexicon. DH Books, Milborne Port, England: 373, $526 \mathrm{pp}$.

Kearns, C. A., Inouye, D. W., Waser, N. M. (1998). Endangered mutualisms: the conservation of plant-pollinator interactions. Annual review of ecology and systematics, 83-112.

Larrea-Alcázar D.M., López R.P. (2011) Pollination biology of Oreocereus celsianus (Cactaceae), a columnar cactus inhabiting the high subtropical Andes. Plant Systematics and Evolution 295: 129-137.

Mandujano M.C., Carrillo-Angeles I., Martínez-Peralta C., Golubov J. (2010) Reproductive biology of Cactaceae. En: Ramawat K.J. (ed.) Desert Plants. Springer-Verlag, Berlin, Germany: 157-171.

Mc Gregor S.E., Alcorn S.M., Olin G. (1962) Pollination and Pollinating Agents of the Saguaro. Ecology 43: 259-267.

Narosky T., Yzurieta D. (2010) Guía para la identificación de las aves de Argentina y Uruguay. Vazquez Mazzini Editores, Buenos Aires, Argentina: 427 pp.

Ollerton J. (1996) Reconciling ecological processes with phylogenetic patterns: The apparent paradox of plant-pollinator systems. Journal of Ecology 84: 
767-769.

Ollerton J., Killick A., Lamborn E., Watts S., Whiston M. (2007) Multiple meanings and modes: on the many ways to be a generalist flower. Taxon 53: 717-728.

Ollerton J., Alarcón R., Waser N.M., Price M.V., Watts S., Cranmer L., Hingston A., Peter C.I., Rotenberry J. (2009) A global test of the pollination syndrome hypothesis. Annals of Botany 103: 1471-1480.

Olson D.M., E. Dirnestein. (2002) The global 200: Priority ecoregions for global conservation. Annals Missouri Botanical Garden 89: 129-224.

Ortega-Baes P., Shüring S., Sajama J., Sotola E., Alonso Pedano M., Bravo S., Godínez-Alvarez H. (2010) Diversity and conservation in the cactus family. En: Ramawat K. (ed.) Desert Plants. Springer.

Ortega-Baes P., Saravia M., Suhring S., Godínez-Álvarez H., Zamar M. (2011) Reproductive biology of Echinopsis terschekii (Cactaceae): the role of nocturnal and diurnal pollinators. Plant Biology 13: 33-40.

Ortega-Baes P., Gorostiague P. (2013) Extremely reduced sexual reproduction in the clonal cactus Echinopsis thelegona. Plant Systematics and Evolution 299: 785-791.

Ortega-Baes P., Godínez-Alvarez, H., Sajama, J., Gorostiague, P., Sühring, S., Galíndez, G., Bravo S., López- Spahr D., Alonso- Pedano M., LindowLópez L., Barrionuevo A., Sosa C., Curti R.N., Juárez A (2015) La familia Cactaceae en Argentina: patrones de diversidad y prioridades políticas para su conservación. Boletín de la Sociedad Argentina de Botánica 50: 71-78.

Pimienta-Barrios E., del Castillo R.F. (2002) Reproductive biology. En: Nobel P.S. (ed.) Cacti: biology and uses. University of California Press, Los Angeles, USA: 163-183.

Rosas-Guerrero V., Aguilar, R., Martén-Rodríguez S., Ashworth L., LopezaraizaMikel M., Bastida J.M., Quesada M. (2014) A quantitative review of pollination syndromes: do floral traits predict effective pollinators?. Ecology Letters, 17(3), 388-400.

Sahley C.T. (1996) Bat and hummingbird pollination of an autotetraploid columnar cactus, Weberbauerocereus weberbaueri (Cactaceae). American Journal of Botany 83: 1329-1336. 
Schlumpberger B.O., Badano E. (2005) Diversity of floral visitors to Echinopsis atacamensis subsp. pasacana (Cactaceae). Haseltonia 11: 18-26.

Schlumpberger B.O., Raguso R. A. (2008) Geographic variation in floral scent of Echinopsis ancistrophora (Cactaceae); evidence for constraints on hawkmoth attraction. Oikos, 117(6), 801-814.

Schlumpberger B.O., Cocucci A.A., Moré M., Sérsic A.N., Raguso R.A. (2009) Extreme variation in floral characters and its consequences for pollinator attraction among populations of an Andean cactus. Annals of Botany 103: 1489-1500.

Schlumpberger B.O., Renner S.S. (2012) Molecular phylogenetics of Echinopsis (Cactaceae): Polyphyly at all levels and convergent evolution of pollination modes and growth forms. American Journal of Botany 99: 1335-1349.

Stebbins G.L. (1970) Adaptive radiation of reproductive characteristics in angiosperms I: Pollination mechanisms. Annual Review of Ecology and Systematics 1: 307-26.

Torres-Díaz C., Gomez-Gonzalez S., Stotz G.C., Torres-Morales P., Paredes B., Pérez-Millaqueo M., Gianoli, E. (2011) Extremely long-lived stigmas allow extended cross-pollination opportunities in a high Andean plant. PloS one 6: e19497.

van der Niet T., Johnson S.D. (2012) Phylogenetic evidence for pollinator-driven diversification of angiosperms. Trends in Ecology \& Evolution 27: 353-361.

Walter H.E. (2010) Floral biology of Echinopsis chiloensis ssp. chiloensis (Cactaceae): evidence for a mixed pollination syndrome. Flora 205: 757763.

Waser N.M., Chittka L., Price M.V., Williams N.M., Ollerton J. (1996) Generalization in pollination systems and why it matters. Ecology 77: 104360. 
Tabla 1. Características florales, sistema reproductivo y gremios polinizadores de las especies de la tribu Trichocereeae del noroeste de Argentina para las que se han realizado estudios sobre su biología reproductiva. Se incluyen las especies estudiadas en el presente trabajo y las publicadas previamente. Sistema Reproductivo: Auto-imcompatible (Al).

\begin{tabular}{|c|c|c|c|c|c|c|c|c|c|c|}
\hline Especies & $\begin{array}{l}\text { Siste } \\
\text { ma } \\
\text { Repro } \\
\text { ductiv } \\
\text { o } \\
\end{array}$ & $\begin{array}{l}\text { Forma } \\
\text { floral }\end{array}$ & Color & $\begin{array}{l}\text { Longitud } \\
\text { promedio de } \\
\text { la corola } \\
(\mathrm{mm})\end{array}$ & $\begin{array}{l}\text { Diámetro } \\
\text { promedio } \\
\text { de la } \\
\text { corola } \\
(\mathrm{mm})\end{array}$ & $\begin{array}{l}\text { Tiempo } \\
\text { de } \\
\text { antesis }\end{array}$ & $\begin{array}{l}\text { Duración de } \\
\text { antesis }\end{array}$ & $\begin{array}{l}\text { Síndrome } \\
\text { floral }\end{array}$ & $\begin{array}{l}\text { Visitantes } \\
\text { (gremios) }\end{array}$ & Referencia \\
\hline $\begin{array}{l}\text { Cleistocactus } \\
\text { baumannii }\end{array}$ & $\mathrm{Al}$ & Tubular & Rojo & $50.20 \pm 9.53$ & $7.19 \pm 1.68$ & Diurna & $\begin{array}{l}\text { Dos días } \\
\text { completos }\end{array}$ & Orintófilo & Picaflores & presente trabajo \\
\hline $\begin{array}{l}\text { C. } \\
\text { smaragdiflorus }\end{array}$ & Al & Tubular & $\begin{array}{l}\text { Magen } \\
\text { ta }\end{array}$ & $44.71 \pm 4.09$ & $6.97 \pm 1.89$ & Diurna & $\begin{array}{l}\text { Dos días } \\
\text { completos }\end{array}$ & Orintófilo & $\begin{array}{l}\text { Picaflores, } \\
\text { Abejas }\end{array}$ & presente trabajo \\
\hline $\begin{array}{l}\text { Denmoza } \\
\text { rhodacantha }\end{array}$ & Al & Tubular & $\begin{array}{l}\text { Rojo- } \\
\text { Magen } \\
\text { ta }\end{array}$ & $49.1 \pm 7.9$ & $9.9 \pm 2.4$ & Diurna & $\begin{array}{l}\text { Dos días } \\
\text { completos }\end{array}$ & Ornitófilo & Picaflores & presente trabajo \\
\hline $\begin{array}{l}\text { Echinopsis } \\
\text { albispinosa }\end{array}$ & $\mathrm{Al}$ & Embudo & Blanco & $128.6 \pm 18.6$ & $78.7 \pm 13.6$ & Nocturna & $\begin{array}{l}\text { Extendida a la } \\
\text { mañana } \\
\text { siguiente }\end{array}$ & Esfingófilo & Abejas & presente trabajo \\
\hline E. ancistrophora & $\mathrm{Al}$ & Embudo & Blanco & $111.7 \pm 17.4$ & $80.0 \pm 14.1$ & Nocturna & $\begin{array}{l}\text { Extendida a la } \\
\text { mañana } \\
\text { siguiente }\end{array}$ & Esfingófilo & $\begin{array}{l}\text { Abejas, } \\
\text { Mariposas } \\
\text { Nocturnas }\end{array}$ & $\begin{array}{l}\text { Schlumpberger } \\
\text { et al. } 2009\end{array}$ \\
\hline E. atacamensis & $\mathrm{Al}$ & Embudo & Blanco & $124 \pm 2$ & $96 \pm 3$ & Diurna & $\begin{array}{l}\text { Extendida a la } \\
\text { mañana } \\
\text { siguiente }\end{array}$ & $\begin{array}{l}\text { Esfingófilo- } \\
\text { Ornitófilo }\end{array}$ & $\begin{array}{l}\text { Picaflores, } \\
\text { Abejas, Polillas, } \\
\text { Avispas }\end{array}$ & $\begin{array}{l}\text { de Viana et al., } \\
2001 ; \\
\text { Schlumpberger } \\
\text { y Badano, } 2005\end{array}$ \\
\hline E. haematantha & $\mathrm{Al}$ & Tazón & $\begin{array}{l}\text { Amarill } \\
\text { o- } \\
\text { Naranj } \\
\text { a }\end{array}$ & $33.5 \pm 4.0$ & $52.1 \pm 6.2$ & Diurna & $\begin{array}{l}\text { Exlusivamente } \\
\text { diurna, abre dos } \\
\text { días }\end{array}$ & Melitófilo & Abejas & presente trabajo \\
\hline E. leucantha & $\mathrm{Al}$ & Embudo & Blanco & $\begin{array}{l}198.17 \pm \\
13.20\end{array}$ & $\begin{array}{l}113.62 \pm \\
21.74\end{array}$ & Nocturna & $\begin{array}{l}\text { Extendida a la } \\
\text { mañana } \\
\text { siguiente }\end{array}$ & Esfingófilo & Abejas, Aves & presente trabajo \\
\hline $\begin{array}{l}\text { E. } \\
\text { sckickendantzii }\end{array}$ & $\mathrm{Al}$ & Embudo & $\begin{array}{l}\text { Blanco } \\
\text {-rosa }\end{array}$ & $118.8 \pm 23.0$ & $105-140$ & Nocturna & $\begin{array}{l}\text { Extendida a la } \\
\text { mañana } \\
\text { siguiente }\end{array}$ & Esfingófilo & $\begin{array}{l}\text { Abejas, } \\
\text { Mariposas } \\
\text { Nocturnas }\end{array}$ & $\begin{array}{l}\text { Alonso-Pedano } \\
\text { y Ortega-Baes } \\
2012\end{array}$ \\
\hline E. terscheckii & $\mathrm{Al}$ & Embudo & Blanco & $177.7 \pm 16.0$ & $\begin{array}{l}163.8 \pm \\
21.7\end{array}$ & Nocturna & $\begin{array}{l}\text { Extendida a la } \\
\text { mañana }\end{array}$ & Esfingófilo & $\begin{array}{l}\text { Abejas, } \\
\text { Mariposas }\end{array}$ & $\begin{array}{l}\text { Ortega-Baes et } \\
\text { al. } 2011\end{array}$ \\
\hline
\end{tabular}


siguiente

\begin{tabular}{|c|c|c|c|c|c|c|c|c|c|c|}
\hline E. thelegona & $\mathrm{Al}$ & Embudo & Blanco & $182.5 \pm 11.5$ & $\begin{array}{l}142.1 \pm \\
11.1\end{array}$ & Nocturna & $\begin{array}{l}\text { Extendida a la } \\
\text { mañana }\end{array}$ & Esfingófilo & $\begin{array}{l}\text { Coleópteros } \\
\text { Abejas, } \\
\text { Mariposas }\end{array}$ & $\begin{array}{l}\text { Ortega-Baes y } \\
\text { Gorostiague }\end{array}$ \\
\hline E. thionantha & $\mathrm{Al}$ & Tazón & $\begin{array}{l}\text { Blanco } \\
- \\
\text { Amarill } \\
\text { o }\end{array}$ & $32.8 \pm 5.5$ & $41.5 \pm 4.6$ & Diurna & $\begin{array}{l}\text { siguiente } \\
\text { Exlusivamente } \\
\text { diurna, abre dos } \\
\text { días }\end{array}$ & Melitófilo & $\begin{array}{l}\text { Nocturnas } \\
\text { Abejas }\end{array}$ & $\begin{array}{l}2013 \\
\text { presente trabajo }\end{array}$ \\
\hline $\begin{array}{l}\text { Gymnocalycium } \\
\text { saglionis }\end{array}$ & $\mathrm{Al}$ & Tazón & $\begin{array}{l}\text { Blanco } \\
\text { - Rosa }\end{array}$ & $27.9 \pm 1.5$ & $24.8 \pm 5.3$ & Diurna & $\begin{array}{l}\text { Exlusivamente } \\
\text { diurna, abre tres } \\
\text { días }\end{array}$ & Melitófilo & Abejas & presente trabajo \\
\hline
\end{tabular}


Tabla 2. Especies de animales polinizadores de las especies de cactáceas de la tribu Trichocereeae del noroeste de Argentina seleccionadas para este estudio.

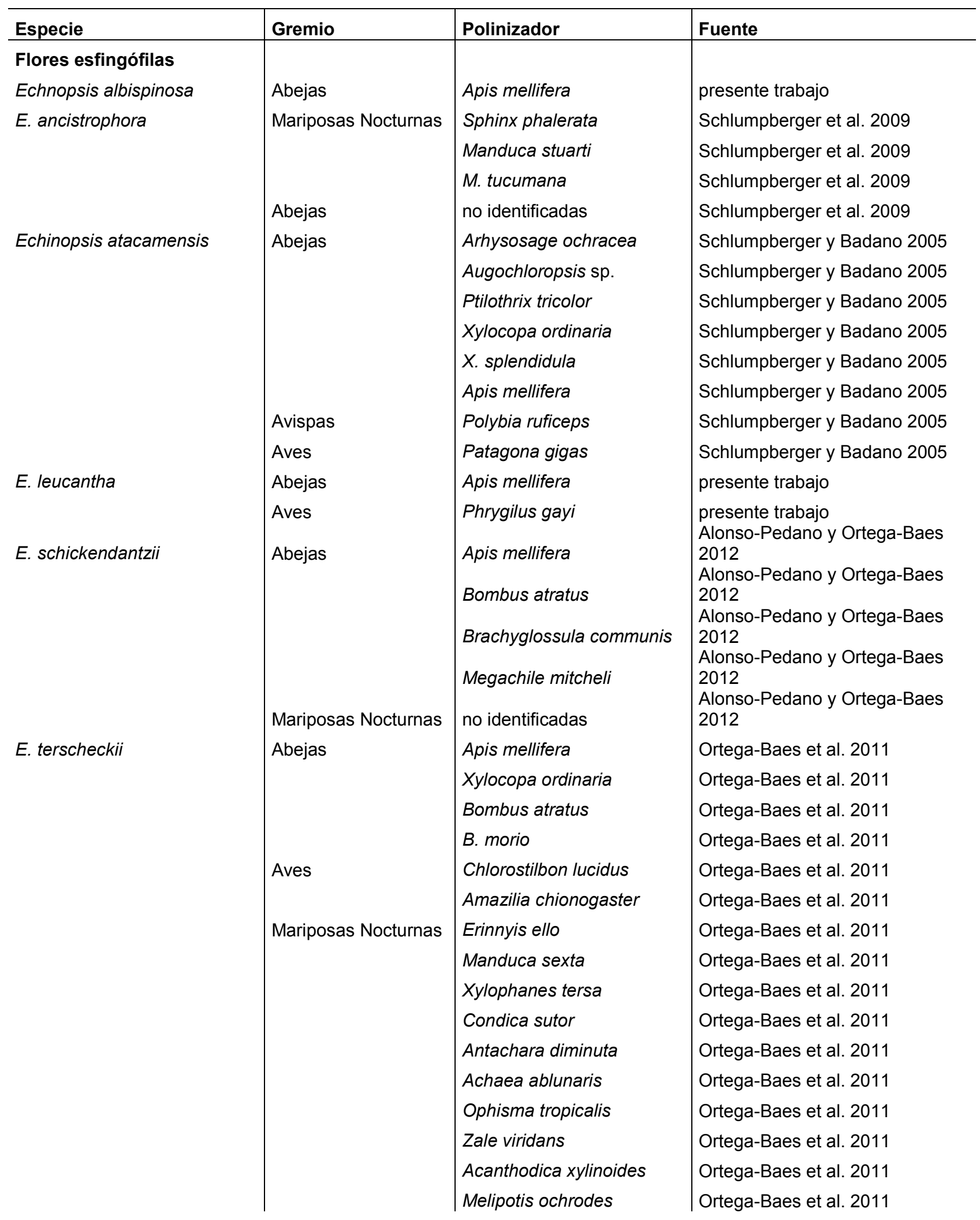




\begin{tabular}{|c|c|c|c|}
\hline E. thelegona & Abejas & $\begin{array}{l}\text { Apis mellifera } \\
\text { Bombus atratus } \\
\text { B. morio } \\
\text { Xylocopa ordinaria }\end{array}$ & $\begin{array}{l}\text { Ortega-Baes y Gorostiague } 2013 \\
\text { Ortega-Baes y Gorostiague } 2013 \\
\text { Ortega-Baes y Gorostiague } 2013 \\
\text { Ortega-Baes y Gorostiague } 2013\end{array}$ \\
\hline \multicolumn{4}{|l|}{ Flores ornitófilas } \\
\hline Cleistocactus baumannii & Aves & $\begin{array}{l}\text { Chlorostilbon lucidus } \\
\text { Colibri coruscans }\end{array}$ & $\begin{array}{l}\text { presente trabajo } \\
\text { presente trabajo }\end{array}$ \\
\hline C. smaragdiflorus & $\begin{array}{l}\text { Aves } \\
\text { Abejas }\end{array}$ & $\begin{array}{l}\text { Chlorostilbon lucidus } \\
\text { Colibri coruscans } \\
\text { Xylocopa sp. }\end{array}$ & $\begin{array}{l}\text { presente trabajo } \\
\text { presente trabajo } \\
\text { presente trabajo }\end{array}$ \\
\hline $\begin{array}{l}\text { Denmoza rhodacantha } \\
\text { Flores melitófilas }\end{array}$ & Aves & Oreotrochilus leucopleurus & presente trabajo \\
\hline E. haematantha & Abejas & $\begin{array}{l}\text { Trichothurgus sp } \\
\text { Brachyglossula communis } \\
\text { Diadasia patagonica }\end{array}$ & $\begin{array}{l}\text { presente trabajo } \\
\text { presente trabajo } \\
\text { presente trabajo }\end{array}$ \\
\hline E. thionantha & Abejas & no identificadas & presente trabajo \\
\hline Gimnocalycium saglionis & $\begin{array}{l}\text { Abejas } \\
\text { Avispas }\end{array}$ & $\begin{array}{l}\text { Arhysosage sp. } \\
\text { Plebeia sp. } \\
\text { Polybia sp. }\end{array}$ & $\begin{array}{l}\text { presente trabajo } \\
\text { presente trabajo } \\
\text { presente trabajo } \\
\end{array}$ \\
\hline
\end{tabular}



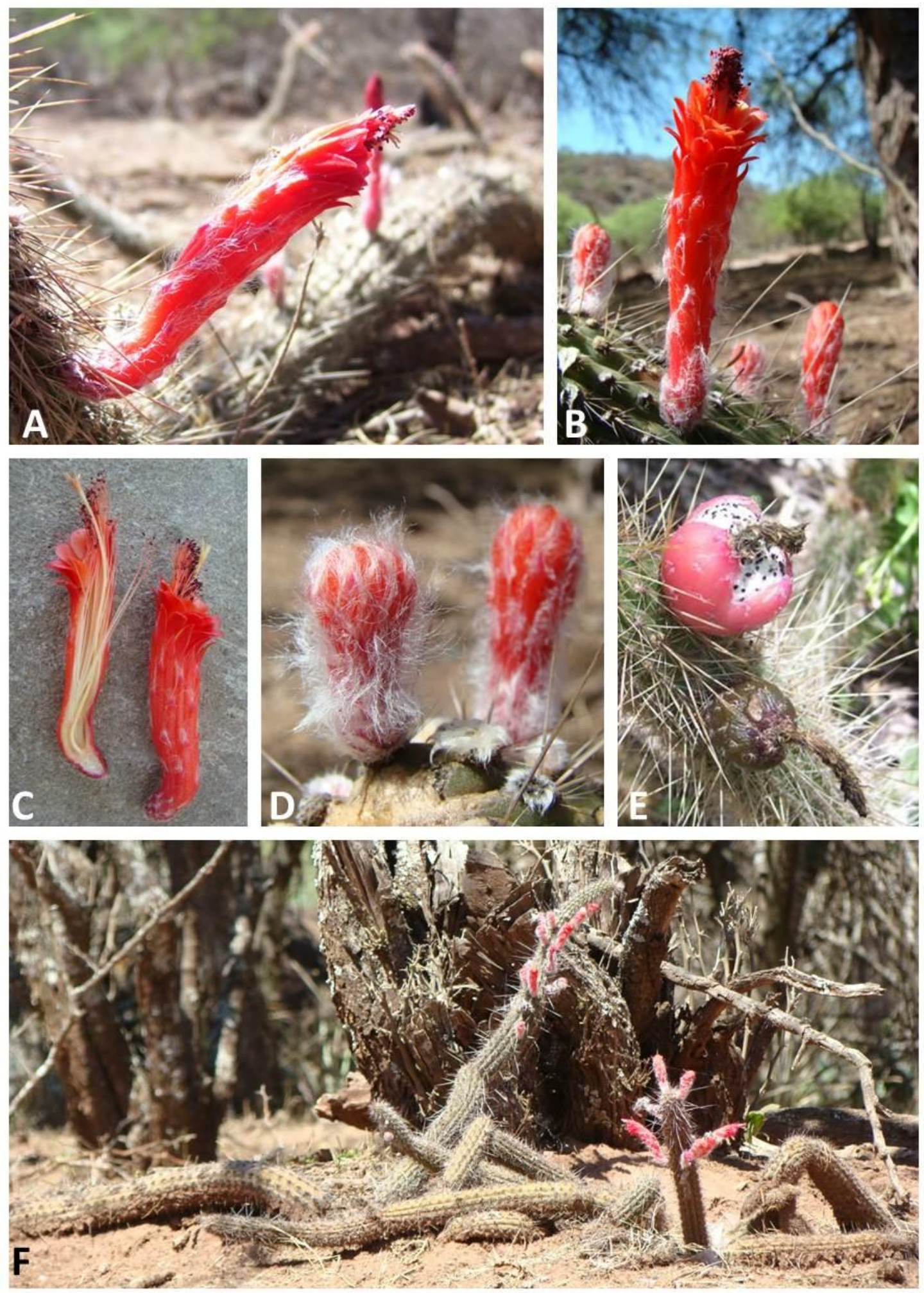

Figura 1. Flores $(A, B, C)$, botones florales $(D)$, frutos $(E)$ e individuos $(F)$ de Cleistocactus baumannii. Fotos: P. Gorostiague. 

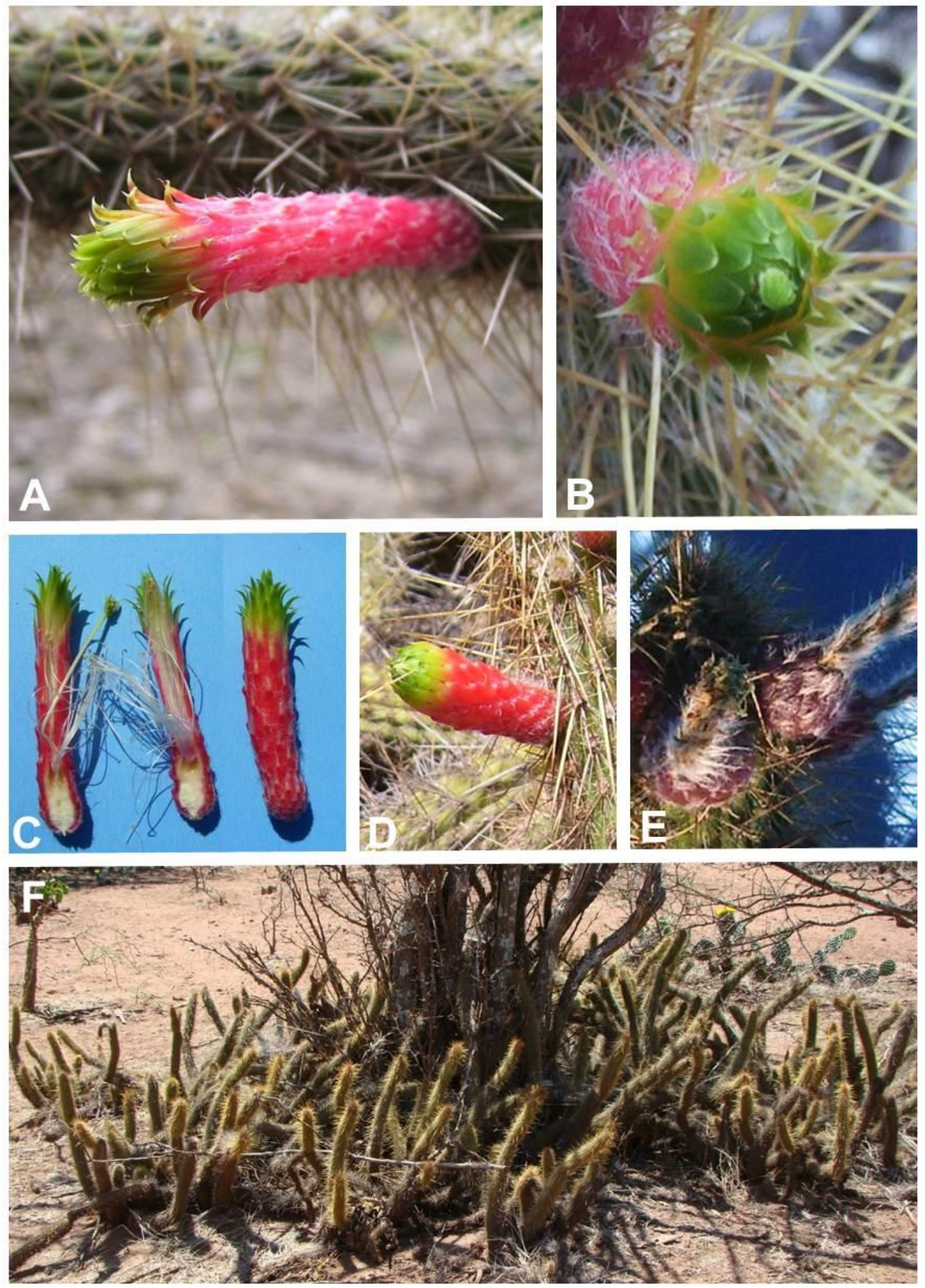

Figura 2. Flores $(A, B, C)$, botón floral $(D)$, frutos $(E)$ e individuos $(F)$ de Cleistocactus smaragdiflorus. Fotos: P. Gorostiague (A,C,D,E,F), P. Ortega-Baes (B). 

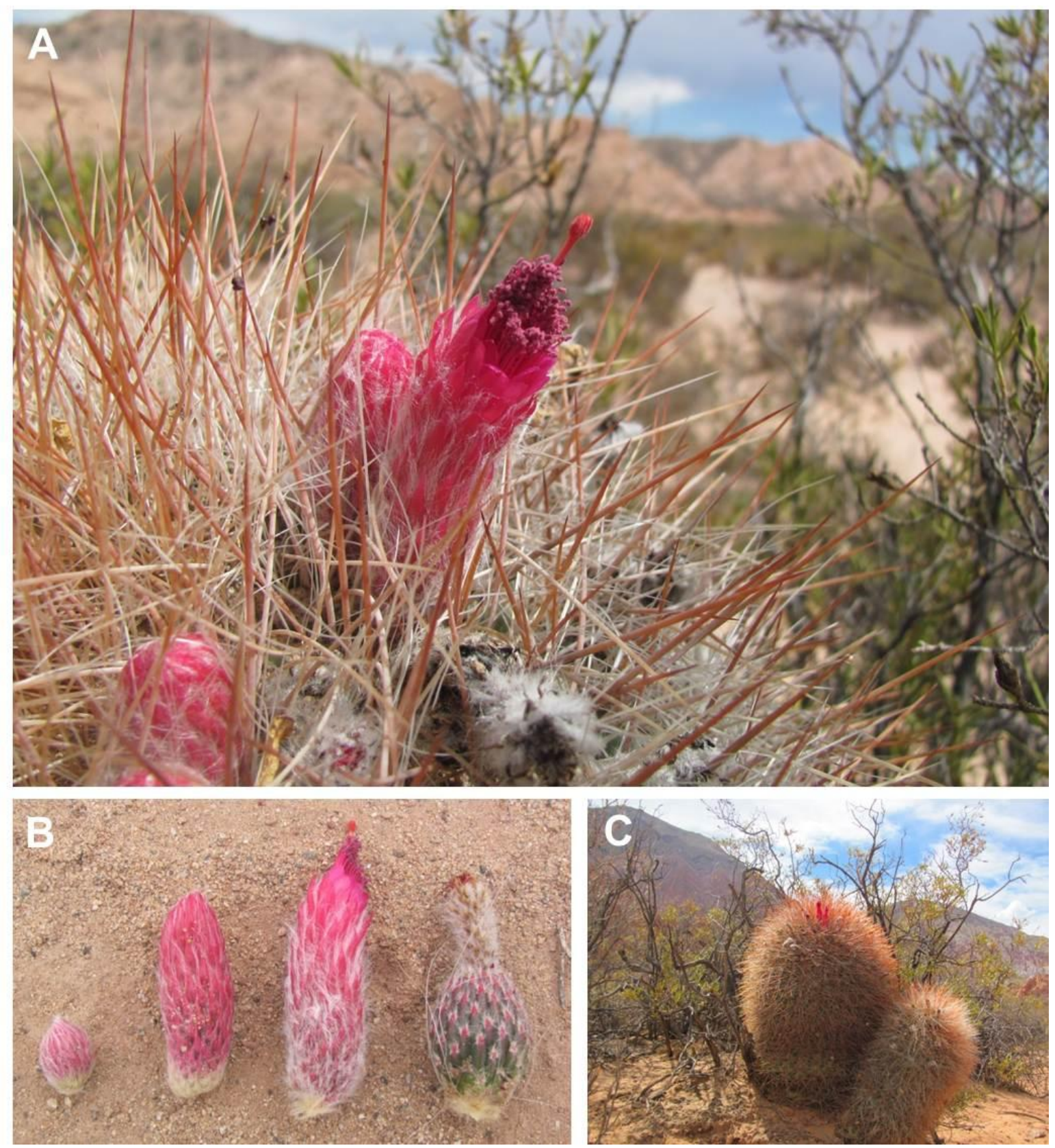

Figura 3. Flores (A, B), botón floral, fruto (B) e individuos (C) de Denmoza rhodacantha. Fotos: P. Gorostiague. 

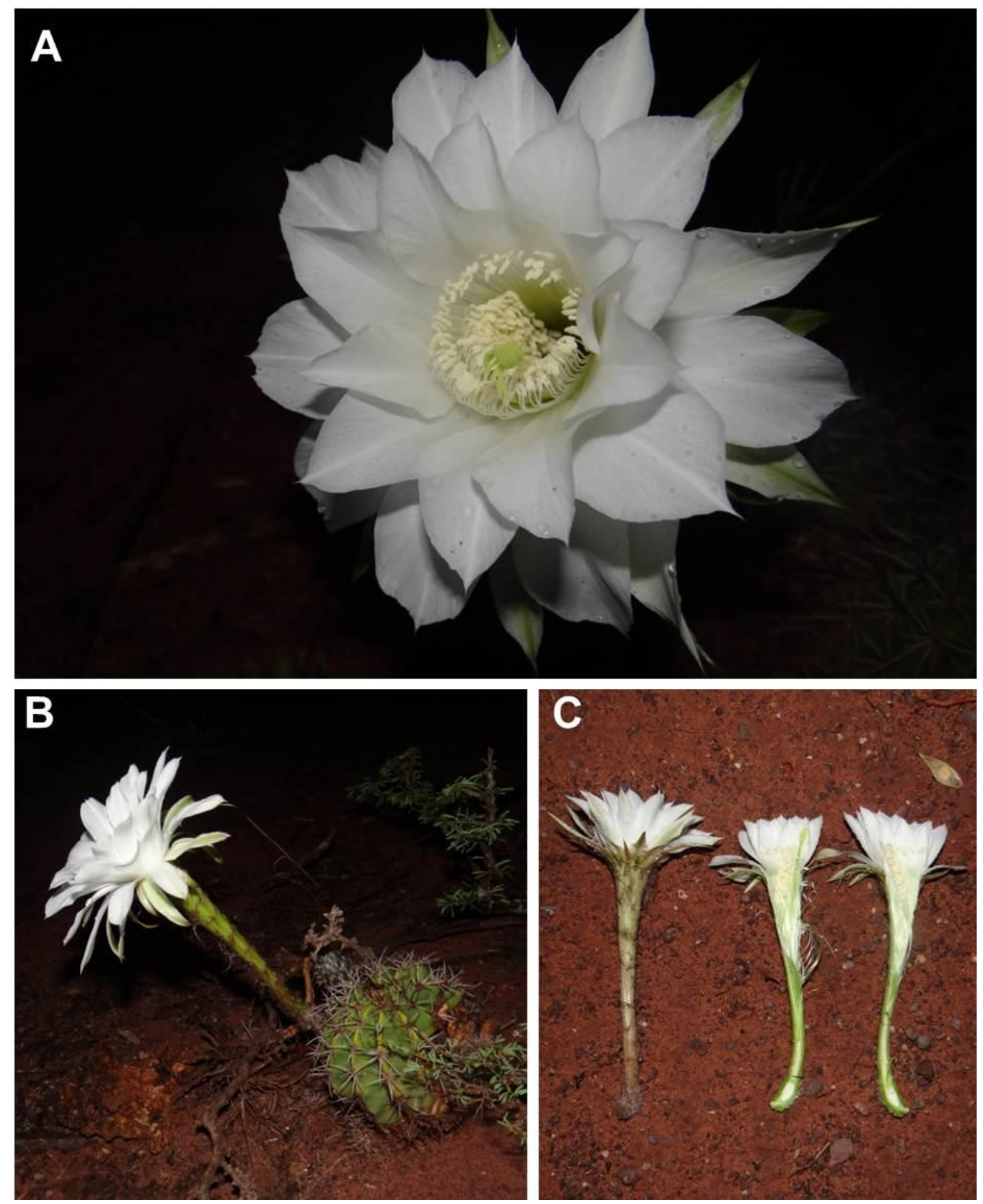

Figura 4. Flores (A, C) e individuos con flor (B) de Echinopsis albispinosa. Fotos: P. Gorostiague. 

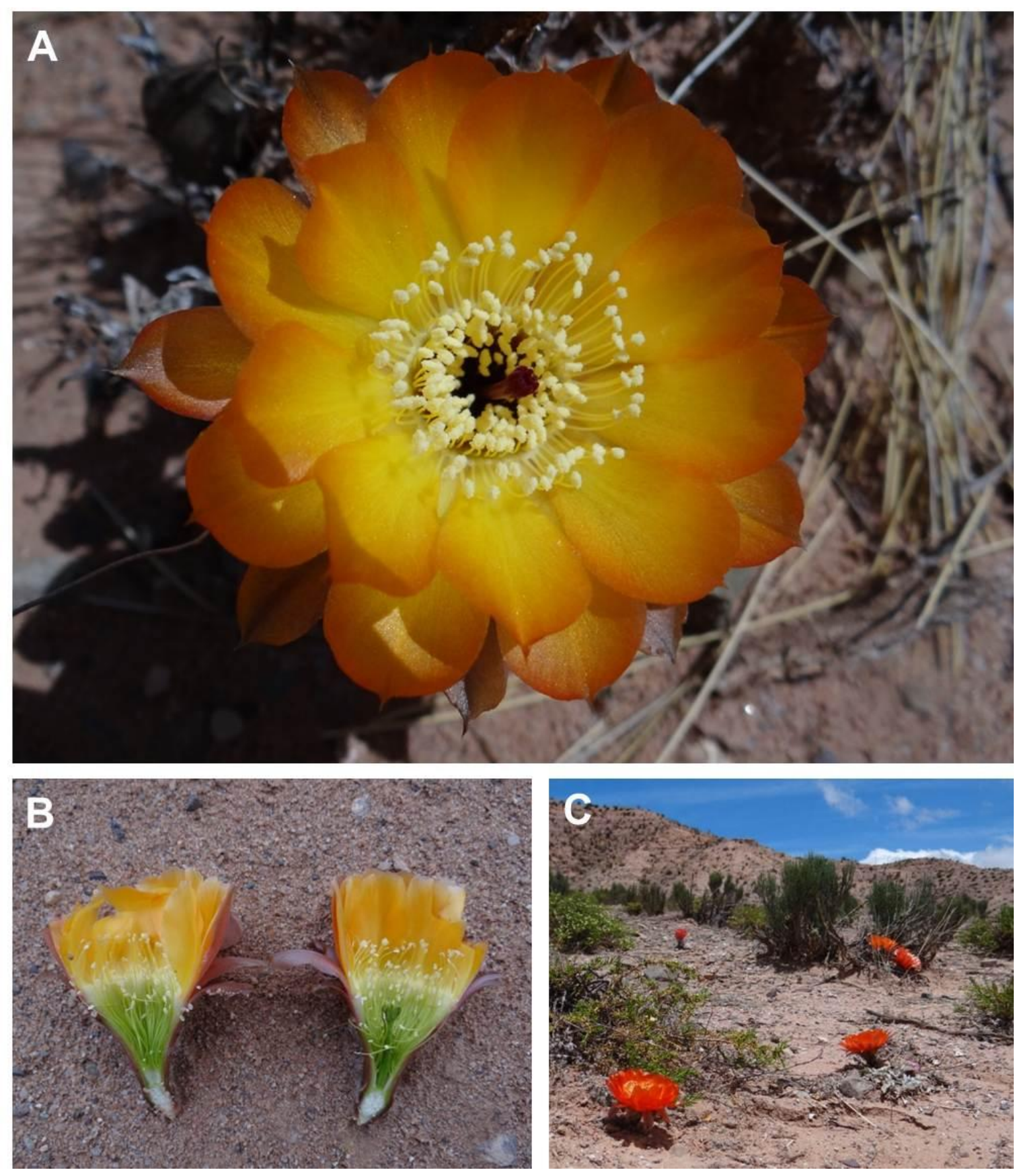

Figura 5. Flores (A, B) e individuos con flor (C) de Echinopsis haematantha. Fotos: P. Gorostiague. 

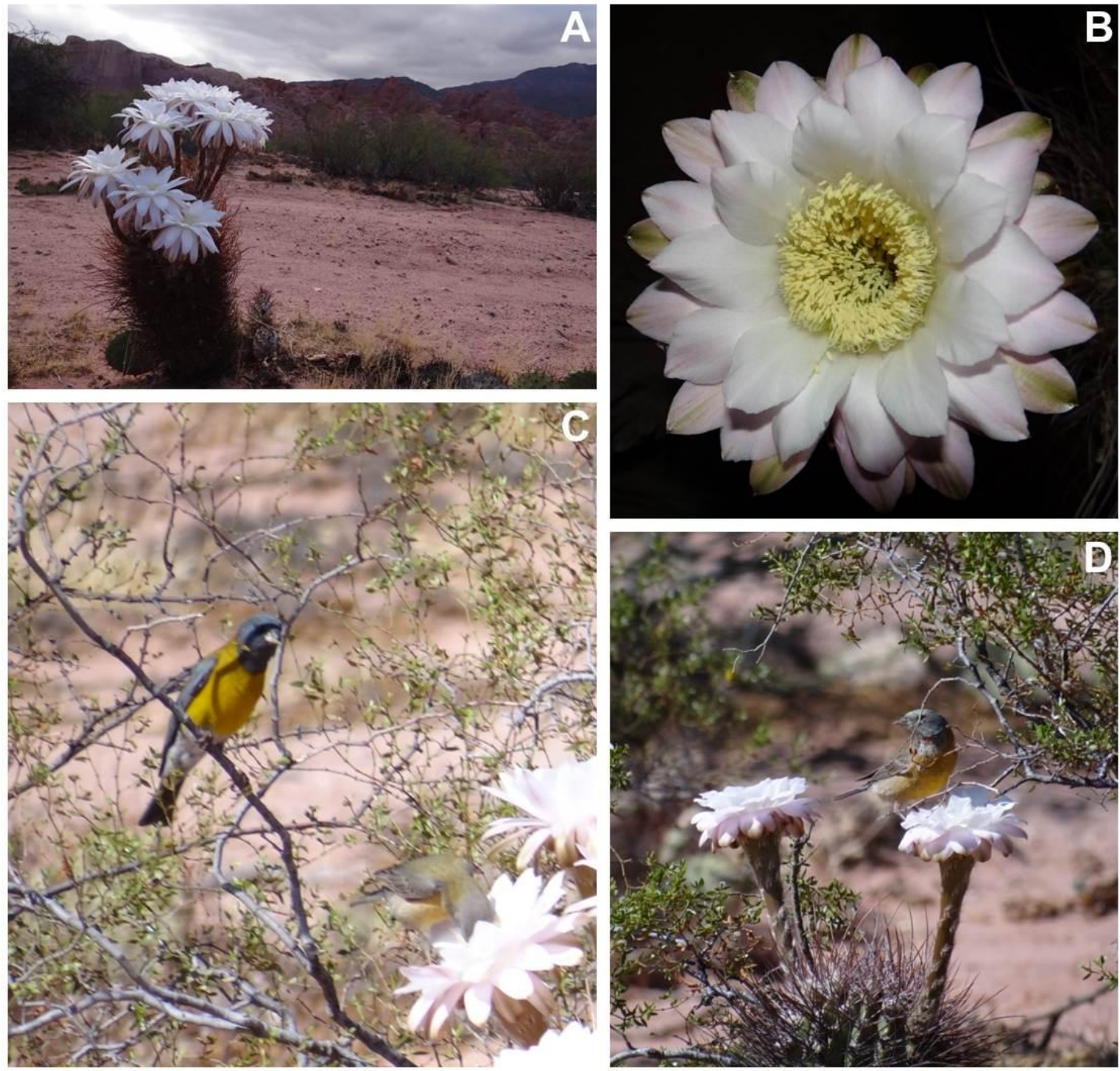

Figura 6. Individuo con flores (A) y flor (B) de Echinopsis leucantha. El ave

Phrygilus gayi (Passeriformes) visitando las flores de esta cactácea (C, D). Fotos: P. Gorostiague. 

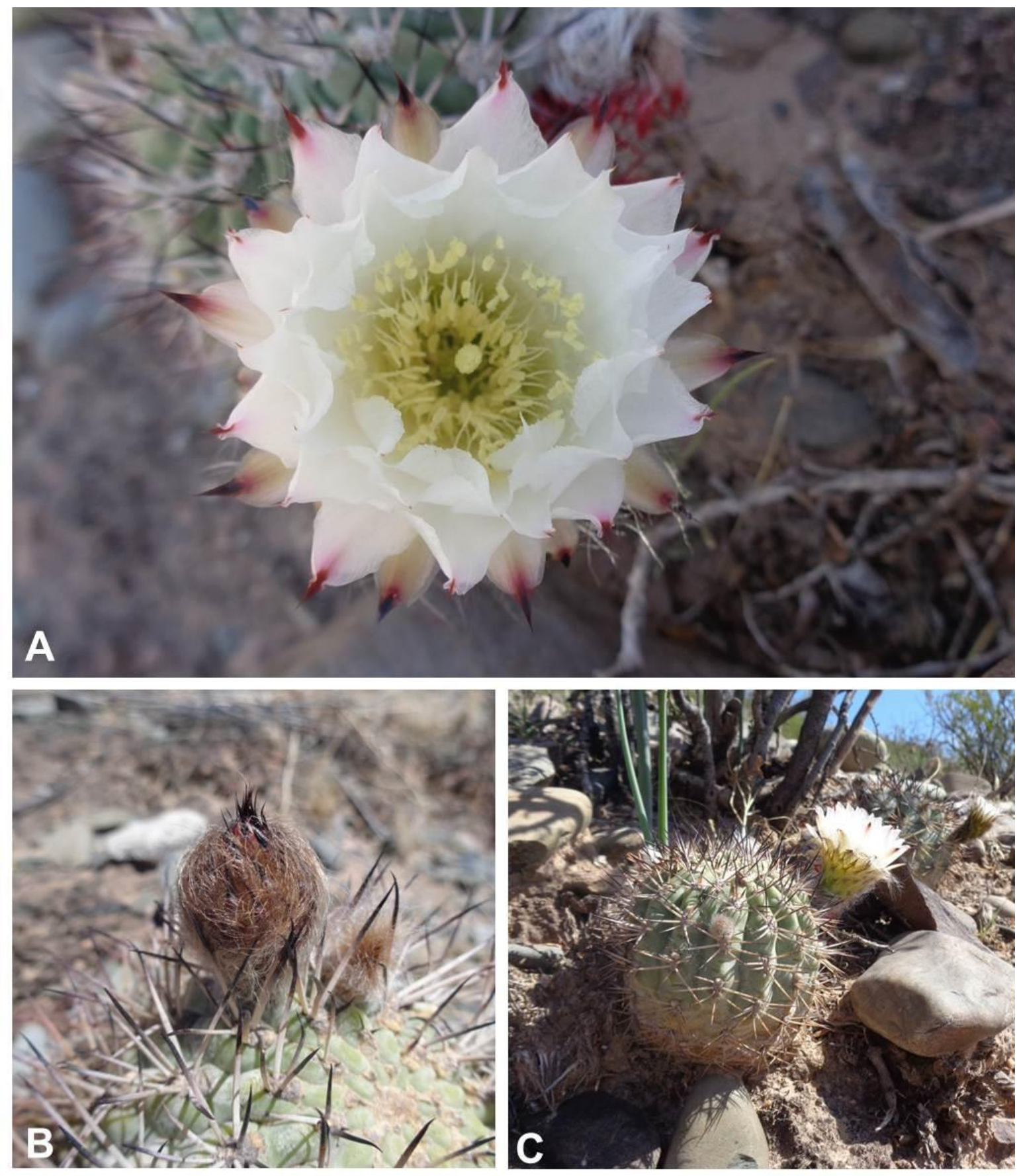

Figura 7. Flor (A), botón floral (B) e individuo con flor (C) de Echinopsis thionantha. Fotos: P. Gorostiague. 

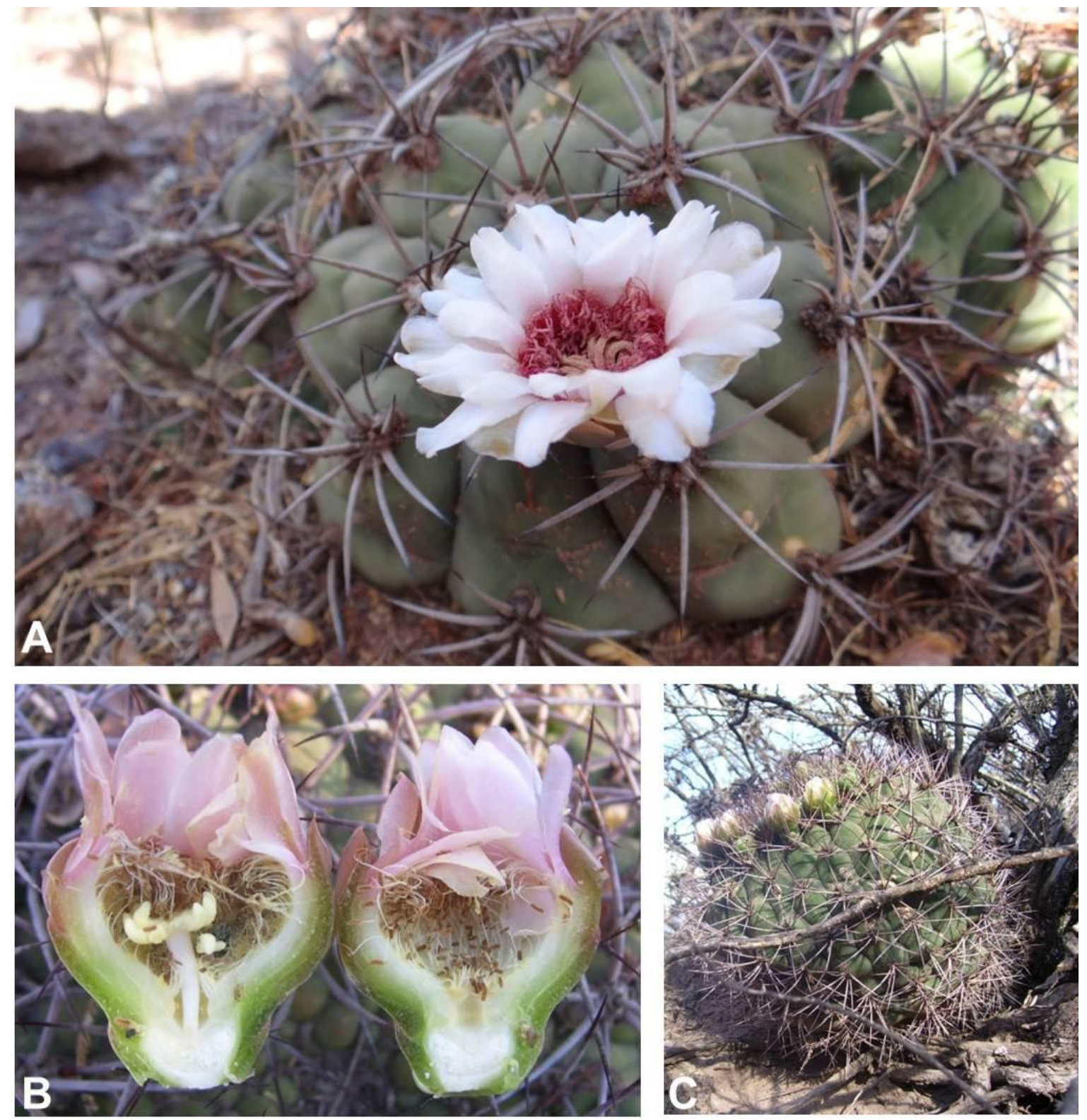

Figura 8. Individuo con flor (A), detalle de flor $(B)$ e individuo con botones florales (C) de Gymnocalycium saglionis. Fotos: P. Gorostiague (A, C), P. Ortega-Baes (B). 


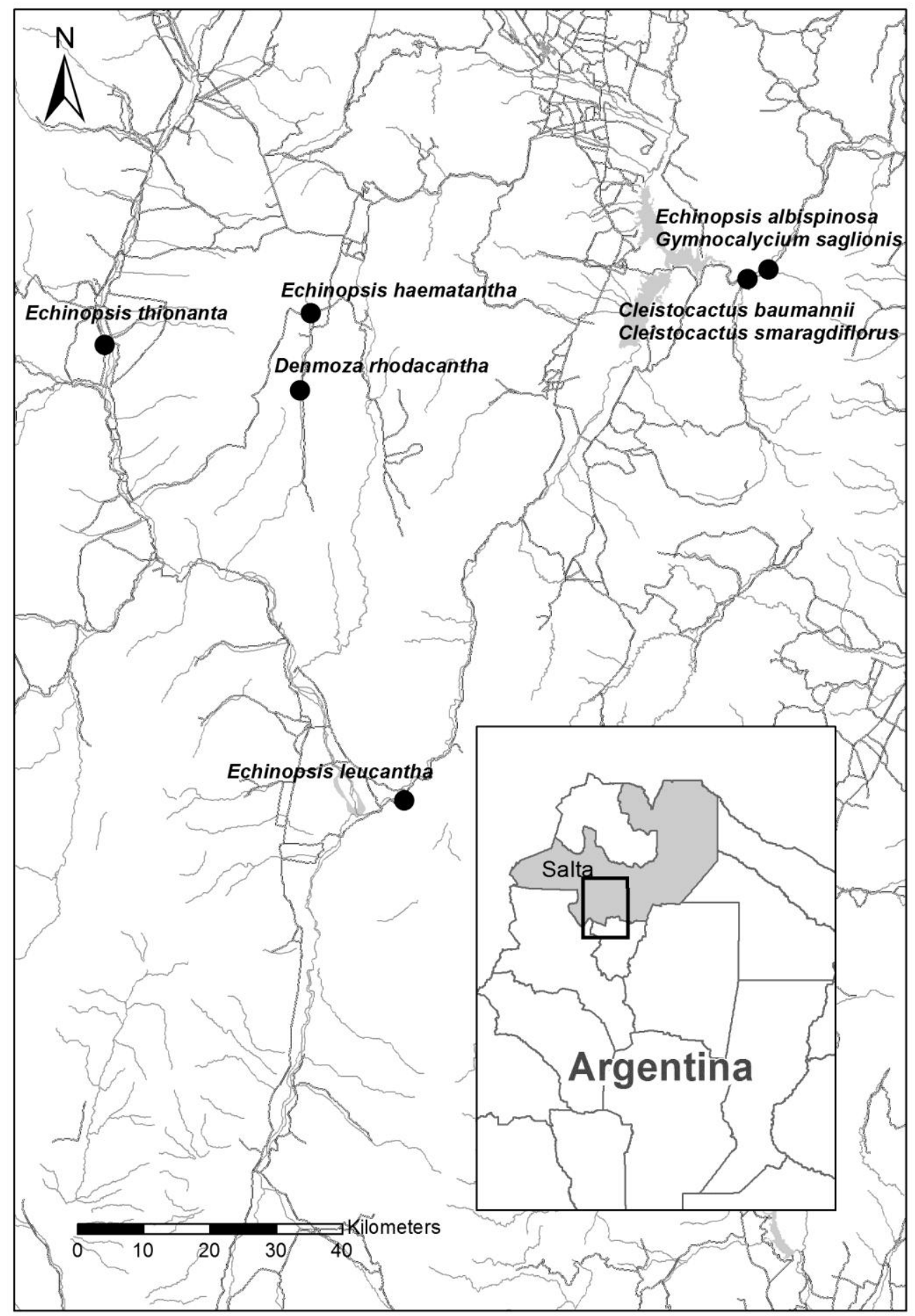

Figura 9. Sitios de estudio donde se realizó el experimento del sistema reproductivo y el muestreo de visitantes florales para las ocho especies incluidas en el estudio. 

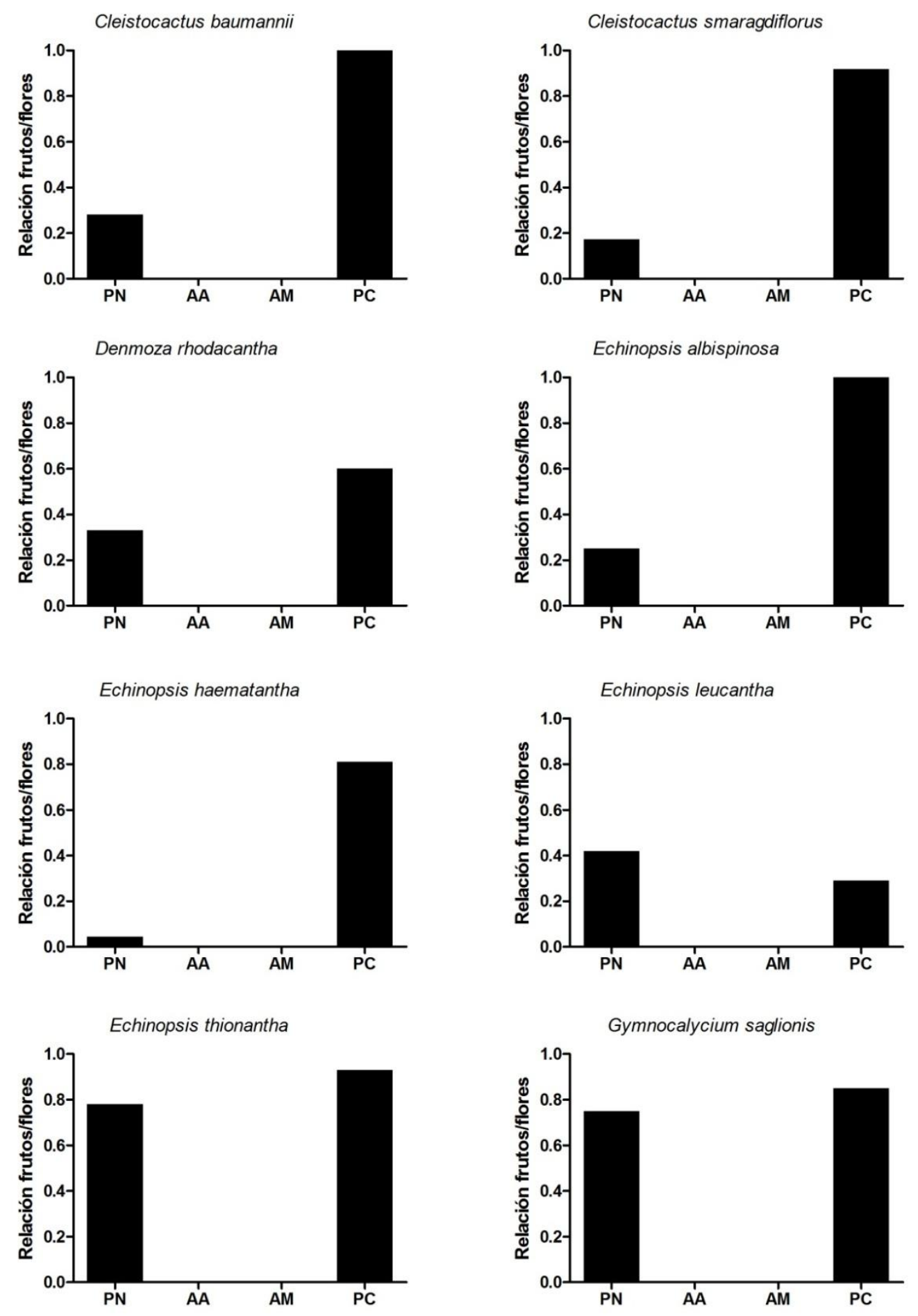

Figura 10. Relación frutos/flores obtenida como resultado del experimento de sistema reproductivo para las ocho especies de cactáceas estudiadas. PN: polinización natural, AA: auto-polinización automática, AM: auto-polinización manual, PC: polinización cruzada manual. 
Cleistocactus baumannii

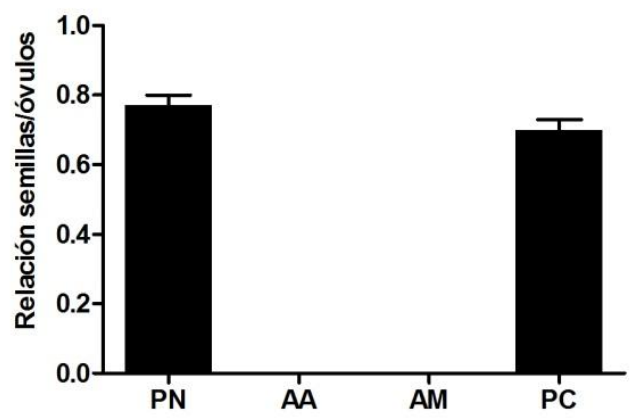

Denmoza rhodacantha

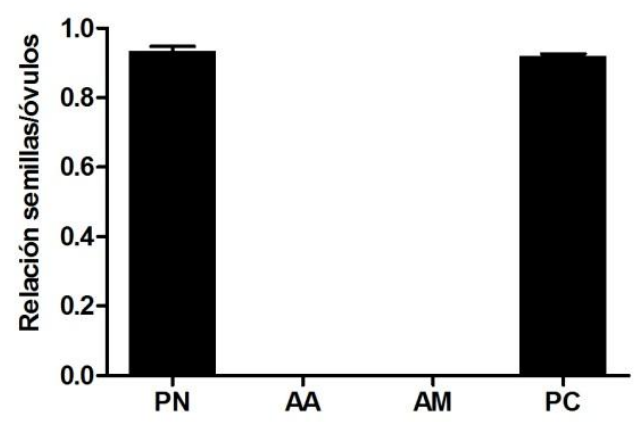

Gymnocalycium saglionis

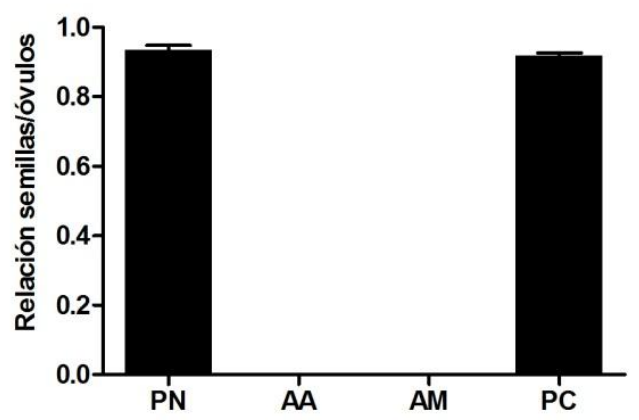

Cleistocactus smaragdiflorus

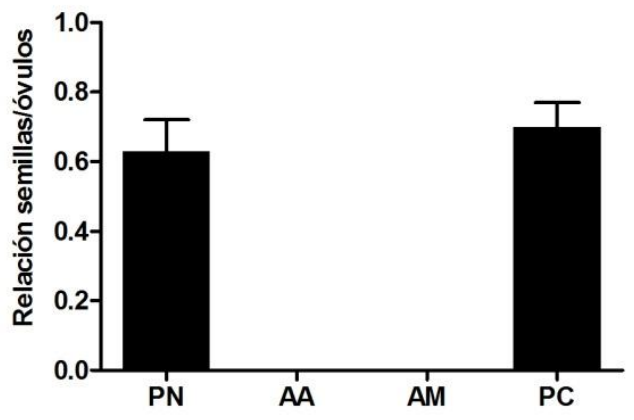

Echinopsis leucantha

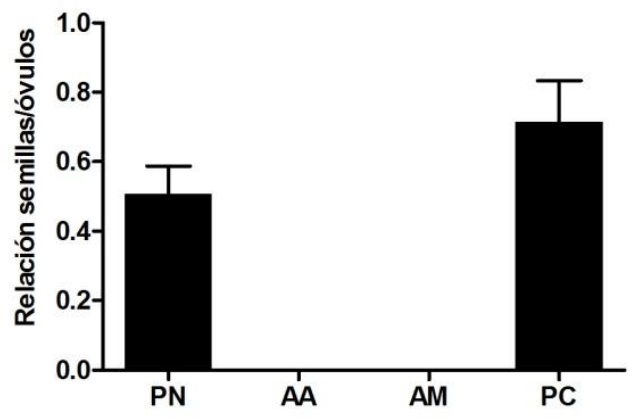

Figura 11. Relación semillas/óvulos (media \pm error estándar) obtenida como resultado del experimento de sistema reproductivo para cinco especies de cactáceas estudiadas. PN: polinización natural, AA: auto-polinización automática, AM: auto-polinización manual, PC: polinización cruzada manual. 

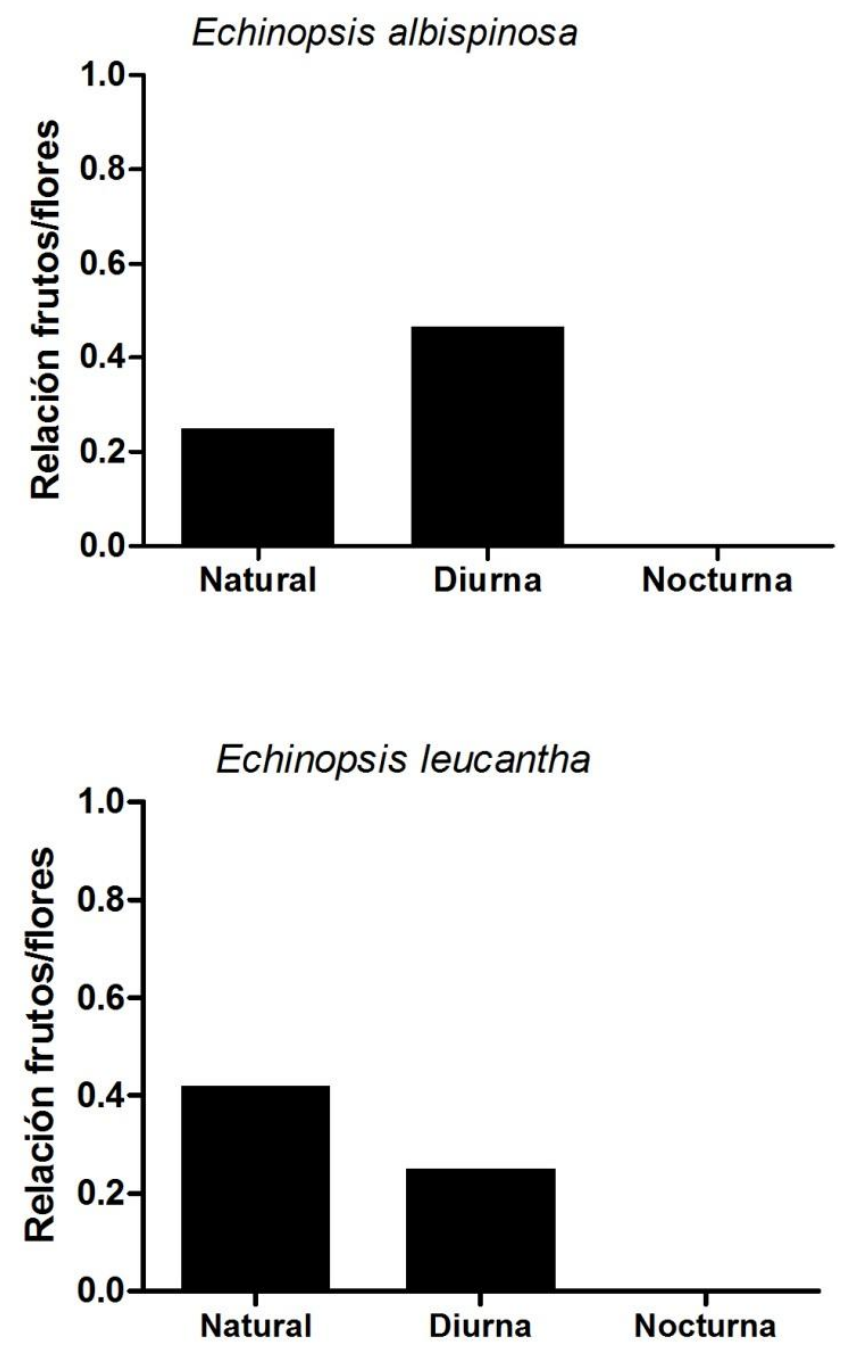

Figura 12. Relación frutos/flores obtenida como resultado del experimento de efectividad de los polinizadores para las especies Echinopsis albispinosa y $E$. leucantha. 


\section{CAPÍTULO II}

¿Qué tan especializados son los sistemas de polinización en la familia Cactaceae?: el caso de la polinización por aves 


\section{INTRODUCCIÓN}

Una concepción común en la biología floral es el entender a los rasgos florales como el resultado de las presiones selectivas de los animales que las polinizan. Generalmente, se cree que los rasgos florales corresponden a vectores de polen específicos. Así, la gran diversidad floral existente podría ser clasificada en diferentes síndromes florales (Faegri y van der Pijl 1979). Si bien este concepto se ha utilizado para predecir los polinizadores principales de numerosas especies de plantas (Stebbins 1970, Fenster et al. 2004), en la mayoría de los casos esta predicción no es acertada debido a que la mayoría de las plantas con flores poseen en realidad sistemas de polinización generalistas (Ollerton 1996, Waser et al. 1996, Ollerton et al. 2009). La mayoría de los estudios basados en el concepto de síndromes florales no tiene en cuenta la dinámica natural dentro de las comunidades más allá de los rasgos florales, tales como las fluctuaciones de las poblaciones de polinizadores, su efectividad, preferencias o interacciones con otras especies. Esta variación a lo largo del tiempo y del espacio y sus efectos sobre la reproducción de las plantas, favorecerían a los sistemas de polinización generalistas más que a los especialistas (Ollerton 1996, Waser et al., 1996, Gómez y Zamora 1999, Hegland y Totland 2005, Ollerton et al. 2007).

Ollerton et al. (2007) propusieron que las flores pueden presentar tres modos diferentes de generalización (o especialización): fenotípica (referida a las adaptaciones que exhibe una flor), funcional (referida a la diversidad de grupos funcionales que polinizan una especie) y ecológica (el número de especies de polinizadores efectivos que interactúa con una especie de planta). Esta diferenciación permite caracterizar a los sistemas de polinización utilizando un enfoque que supera el conflicto conceptual que ocurre cuando se los define simplemente como generalizados o especializados (Waser 2006, Ollerton et al. 2007).

Las flores son una característica muy distintiva de las especies de cactáceas, y muestran una gran diversidad en cuanto a forma, tamaño, color y tiempo de antesis (Gibson y Nobel 1986, Pimienta-Barrios y del Castillo 2002). Tradicionalmente, se ha considerado que los sistemas de polinización en la familia Cactaceae son especializados. Sin embargo, la mayoría de los sistemas 
estudiados hasta la fecha son generalistas, con murciélagos, abejas, mariposas nocturnas y aves como polinizadores efectivos (Fleming et al. 2001, Bustamante et al. 2010, Ortega-Baes et al. 2011, Alonso-Pedano y Ortega-Baes 2012, Capítulo I).

Las flores de aves (o flores ornitófilas) han sido descriptas para un gran número de especies de cactáceas de diferentes linajes, particularmente en América del Sur. En general, en la descripción taxonómica de muchas de estas especies se asume que tienen sistemas de polinización especializados a las aves, principalmente picaflores (Anderson 2001, Hunt 2006). En algunos géneros de cactáceas Sudamericanos, tales como Oreocereus, Cleistocactus, Matucana y Denmoza, las flores ornitófilas son una característica común (Anderson 2001, Hunt 2006), lo cual sugiere que estos linajes evolucionaron hacia la especialización por aves. Sin embargo, existe poca información acerca de la relación entre las aves y las flores de las cactáceas (Díaz y Cocucci 2003, Nassar y Ramírez 2004, Colaço et al. 2006, Saleme Aona et al. 2006, Nassar et al. 2007, Fagua y Ackerman 2011, Larrea-Alcázar y López 2011).

En este Capítulo, se utilizó al género Cleistocactus (Tribu Trichocereeae) como un modelo de estudio para probar la idea de que las cactáceas con flores ornitófilas establecen relaciones especializadas con las aves, determinando qué modo de especialización presentan estas interacciones sensu Ollerton et al. (2007). El género Cleistocactus incluye 37-48 especies distribuidas típicamente en las regiones tropicales de Sudamérica, desde Perú hasta Argentina (Anderson 2001, Hunt 2006). Este género es citado como un ejemplo de extrema especialización a la polinización por aves (Scogin 1985, Rose y Barthlott 1994, Anderson 2001, Hunt 2006); sin embargo, no existen estudios sobre su biología reproductiva. Específicamente, se estudió la biología de la polinización de Cleistocactus baumannii y C. smaragdiflorus, dos especies de cactáceas que son simpátricas a lo largo de una parte de su distribución geográfica en el noroeste de Argentina. Se planteó la hipótesis de que estas dos especies poseen especialización fenotípica y funcional a la polinización por picaflores. Sin embargo, dado que muchas especies de picaflores han sido citadas para la ecorregión del Chaco Seco argentino, donde estas dos especies se distribuyen (Aizen y Feinsinger 1994, Heredia 2011), también se puso a prueba la hipótesis de que 
ambas especies de cactáceas son visitadas por varias especies de picaflores y poseen por lo tanto generalización ecológica. Particularmente, se respondieron las siguientes preguntas: a) ¿poseen las flores de C. baumannii y C. smaragdiflorus especialización fenotípica a la polinización por aves? b) ¿dependen estas especies de sus polinizadores para la producción de frutos y semillas? c) ¿los visitantes corresponden a los rasgos florales? d) ¿qué nivel de especialización sensu Ollerton et al. (2007) presentan estas especies? Adicionalmente, se utilizó el enfoque de Ollerton et al. (2007) para reevaluar el modo de especialización que muestran los sistemas de polinización de cactáceas que incluyen aves, estudiados previamente por otros autores. Finalmente, utilizando la información disponible, se analizó qué tan común es la especialización fenotípica a la polinización por aves entre los diferentes linajes de la familia Cactaceae. Específicamente, se utilizaron diferentes rasgos florales citados como ornitófilos para este análisis, y se consideró al color como la característica más descriptiva de este tipo de flor (Wilson et al. 2004, Curti y Ortega-Baes 2011).

\section{MATERIALES Y MÉTODOS}

\section{Área de estudio}

El estudio se llevó a cabo en La Bodeguita (2517'31.72"S, 65¹6'25.13"O), en el departamento La Viña, en la provincia de Salta (Fig. 1). El área de estudio corresponde a la ecorregión del Chaco Seco. La temperatura media anual en este sitio es de $18.4^{\circ} \mathrm{C}$, mientras que la precipitación media anual es de $416 \mathrm{~mm}$ (Bianchi 1996, Bianchi y Yáñez 1992). Adicionalmente, se buscaron y localizaron otras poblaciones de ambas especies de Cleistocactus en la provincia de Salta para registrar los visitantes florales. Para esto, se utilizó el software MaxEnt (Phillips et al. 2006) para seleccionar diez sitios con una alta probabilidad de ocurrencia $(\geq 0.75$ ) de cada especie.

\section{Especies bajo estudio}

Ambas especies de Cleistocactus incluidas en este estudio son columnares arbustivas, erectos o arqueados que se ramifican en la base (Fig. 2A, 2D). Las flores de Cleistocactus baumannii son rojas, tubulares, ligeramente curvadas con forma de "s" (Fig. 2B). Esta especie produce frutos pequeños esféricos de color rojo con pulpa blanca y semillas negras (Anderson 2001, Hunt 2006). Cleistocactus 
smaragdiflorus posee flores tubulares rectas color rosa, con tépalos apicales verdes (Fig. 2C). Sus frutos son rosados o rojos, esféricos y contienen pulpa blanca y semillas negras. (Anderson 2001, Hunt 2006). Las semillas de ambas especies no presentan dormición (Ortega-Baes et al. 2010) y son fotoblásticas positivas (Lindow-López et al. datos sin publicar).

\section{Morfología floral}

Veinte flores abiertas de $C$. baumannii y 33 de $C$. smaragdiflorus de 10 individuos diferentes fueron colectadas al azar y preservadas en alcohol $70 \%$ para su análisis morfométrico. Los siguientes rasgos florales fueron registrados: diámetro de la corola, largo total de la flor, largo del gineceo, largo del estilo, largo y diámetro del estigma, largo del estambre más corto y más largo, largo y diámetro del ovario, largo y diámetro interno del ovario, largo y diámetro de la cámara nectarial, número de estambres, número de lóbulos estigmáticos y número de óvulos. También se describió cualitativamente el color de la corola y del polen a partir de flores abiertas frescas.

\section{Ciclo floral}

Para determinar el período en el que las flores están disponibles para los polinizadores, se registró el estadío fenológico de 20 flores de cada especie. El registro se llevó a cabo cada dos horas, desde el estadío de botón floral hasta la senescencia.

\section{Producción de néctar y concentración de azúcares}

El volumen de néctar producido por flor fue registrado en 15 flores de cada especie. Las flores fueron cubiertas con bolsas de voile para prevenir el consumo del néctar por parte de los visitantes florales. El mismo se extrajo cada cuatro horas utilizando tubos capilares de $80-\mu \mathrm{L}$. La concentración de azúcares se determinó para diez flores de cada especie cada cuatro horas utilizando un refractómetro de mano de grados brix.

\section{Sistema reproductivo}

Se llevó a cabo un experimento durante 2011 en el sitio La Bodeguita para determinar el sistema reproductivo de $C$. baumannii y $C$. smaragdiflorus. Los tratamientos fueron: 1) polinización natural: flores sin manipular; 2) autopolinización autónoma: flores excluidas sin manipulación; 3) auto-polinización 
manual: flores emasculadas polinizadas manualmente usando polen de la misma flor y polen de otras flores del mismo individuo; y 4) polinización cruzada manual: flores emasculadas y polinizadas manualmente utilizando polen de cinco flores de otros individuos. Las flores de los tratamientos 2), 3) y 4) fueron excluidas de los polinizadores utilizando bolsas de voile luego de aplicarles el tratamiento. El tratamiento de polinización natural fue aplicado nuevamente en 2012 utilizando dos poblaciones adicionales de C. baumannii y C. smaragdiflorus, en áreas habitadas por sólo una de las dos especies. Las variables de respuesta fueron la relación frutos/flores y la relación semillas/óvulos.

\section{Visitantes florales}

El registro de visitantes florales se llevó a cabo mediante observaciones focales sobre cinco flores de $C$. baumannii y $C$. smaragdiflorus, durante 15 minutos cada cuatro horas desde las 07:00 hasta las 18:00 hs. Esta observación se realizó durante siete días no consecutivos a lo largo del período reproductivo en el sitio La Bodeguita, donde estas especies solapan su distribución. Además, se filmaron con una videocámara cuatro flores de $C$. baumannii durante 225 minutos y cuatro flores de $C$. smaragdiflorus durante 150 minutos, distribuidos en tres días no consecutivos. Un muestreo adicional de los visitantes florales se llevó a cabo en el sitio La Bodeguita y en diez sitios adicionales para cada especie seleccionados en base al modelo de distribución de las especies construido con MaxEnt. Sólo fue posible registrar los visitantes florales en tres sitios adicionales para C. baumanni y cuatro sitios adicionales para C. smaragdiflorus (Fig. 1) debido a que el resto de las poblaciones no produjeron flores. En todos los sitios adicionales, las observaciones focales se llevaron a cabo durante dos días no consecutivos. Los insectos que visitaron las flores fueron colectados utilizando redes de mano y luego determinados y depositados en la sección de entomología del Museo Argentino de Ciencias Naturales Bernardino Rivadavia (Buenos Aires). Las abejas fueron registradas como visitantes sólo si ingresaron a las flores. Las aves fueron identificadas con binoculares con ayuda de una guía de campo (Narosky y Yzurieta 2010, Heredia 2011). Debido a que las flores permanecieron abiertas durante la noche, se colectaron 15 estigmas (en tres días no consecutivos) de flores cerradas de cada especie, los cuales fueron analizados posteriormente en el 
laboratorio en busca de escamas de mariposas nocturnas, con el fin de evaluar si estos animales se comportan como visitantes florales nocturnos. Adicionalmente, se montó una trampa de luz UV para capturar mariposas nocturnas desde las 22:00 hasta las 01:00 hs durante tres días no consecutivos en 2010, 2011 y 2012. Las mariposas capturadas fueron llevadas al laboratorio para su determinación y para extraer muestras de polen de sus cuerpos (Capítulo I).

\section{Modos de especialización a la polinización por aves en especies de cactáceas estudiadas previamente}

Para analizar los modos de especialización en estudios previamente publicados de cactáceas polinizadas por aves, se realizó una búsqueda intensiva de artículos científicos utilizando Scopus (http://www.scopus.com) y Google Scholar (http://scholar.google.com) hasta Diciembre de 2013. Para esta búsqueda, se utilizaron combinaciones de las siguientes palabras clave: ("Cactaceae" or "cactus") and ("pollination" or "bird" or "hummingbird"). Adicionalmente, se examinó la bibliografía citada en los artículos obtenidos. A partir de cada especie estudiada, se resumió la información acerca de la forma floral, color, tiempo de antesis, longitud y diámetro de la corola, producción de néctar, concentración de azúcares, síndrome floral y visitantes florales. Se utilizaron los nombres científicos utilizados originalmente por el/los autor/es. Adicionalmente, se incluyó en este análisis a las dos especies de Cleistocactus estudiadas aquí y a dos especies estudiadas en el Capítulo 1 para las cuales se registraron aves como polinizadores: Denmoza rhodacantha y Echinopsis lecucantha. Con el propósito de visualizar la variabilidad entre estos rasgos, se realizó un análisis de coordenadas principales utilizando escalamiento multidimensional (MDS), tal como el utilizado por Wilson et al. (2004). El análisis incluyó siete variables cuantitativas y cualitativas (largo promedio de la flor, ancho promedio de la flor, producción promedio de néctar por flor, concentración promedio de azúcares, tiempo de antesis, forma floral y color de la corola). Las variables fueron estandarizadas, y se utilizó la distancia Euclidea para el análisis. De esta manera, se obtuvo una visualización gráfica de la disimilitud entre las flores de las diferentes especies basándose en rasgos asociados a la polinización. Para poder utilizar el color como una variable cualitativa, cada especie se codificó numéricamente del 1 al 4 , donde 1 
representaba a las flores completamente blancas y 4 representaba a las flores completamente rojas, utilizando los números 2 y 3 para colores intermedios. Para la forma floral, las especies se codificaron del 1 al 3 (1 para flores con forma de tazón, 2 para flores con forma de embudo y 3 para las tubulares). Finalmente, para el ciclo floral, dos categorías fueron consideradas: 1) nocturnas y 2) diurnas. Las flores consideradas nocturnas fueron aquellas que se abrieron durante la noche $y$ tuvieron un ciclo extendido hasta la mañana siguiente, mientras que las flores consideradas diurnas incluyeron a aquellas exclusivamente diurnas (flores de un día) y también a aquellas que se abrieron durante el día y permanecieron abiertas durante más de un día. Para completar los registros faltantes de tamaño floral y concentración de azúcares, se asignaron los valores medios del género, o el valor mediano de la variable en el caso de especies con un solo género representado en el análisis.

\section{Especialización fenotípica a las aves en la familia Cactaceae}

Para analizar qué tan común es la especialización fenotípica a la polinización por aves entre los linajes de la familia Cactaceae, se construyó una base de datos de las 1430 especies reconocidas de cactáceas utilizando la información disponible en Hunt (2006). También se tuvieron en cuenta a todas aquellas subespecies que mostraron diferencias entre sus rasgos florales, alcanzando un total de 1584 registros. Los rasgos florales considerados, además del color, fueron tamaño (largo y diámetro), forma y tiempo de antesis. Se consideraron como flores "rojizas" a todas aquellas que presentaron color rojo, rosa, violeta, naranja y sus combinaciones. Las flores que presentaron combinaciones con otros colores no fueron consideradas rojizas.

Se realizó una prueba de aleatorización para evaluar si el tamaño promedio (largo y diámetro) de las flores rojizas fue significativamente diferente del tamaño promedio de todas las flores de cactáceas. Dado que la distribución de estas variables fue asimétrica, también se evaluaron los valores de la mediana del tamaño. Adicionalmente, se puso a prueba si el porcentaje de flores diurnas y de flores tubulares entre las especies con flores rojizas fue significativamente diferente del porcentaje dentro de toda la familia. Para cada especie, se indicó la correspondiente subfamilia y tribu y el color de sus flores. A partir de estos datos, 
se determinó el porcentaje de especies con flores rojizas en cada grupo. Se construyó una representación de la filogenia de la familia que incluyó a estos grupos, ubicándolos en base a la filogenia propuesta por Bárcenas et al. (2011). A través de una prueba de aleatorización, se evaluó si la proporción de especies con flores rojizas era la esperada por azar en cada grupo taxonómico. Para todas las pruebas de aleatorización, se tomaron 1000 muestras al azar sin reposición y se obtuvieron intervalos de confianza del 95\%.

\section{Análisis estadístico}

El largo promedio del gineceo y el largo promedio del estambre más largo fueron comparados a través de una prueba $t$, con el fin de probar la excerción del estigma. La producción de néctar y la concentración de azúcares fueron comparadas entre las especies de Cleistocactus utilizando una prueba $t$. Los valores de la relación frutos/flores entre tratamientos del experimento de sistema reproductivo fueron comparadas usando una prueba exacta de Fisher, mientras que los valores de la relación semillas/óvulos fueron comparados con una prueba $t$. Todos los análisis estadísticos fueron realizados utilizando el software Infostat versión 2009 (Di Rienzo et al. 2009), a excepción de las pruebas de aleatorización que fueron realizadas utilizando el software $R$ ( $R$ Development Core Team 2012).

\section{RESULTADOS}

\section{Morfología floral}

Las flores de Cleistocactus baumannii fueron tubulares, ligeramente curvadas, con corola de color rojo y polen rosa o rojo (Fig. 2B). Éstas tuvieron un largo promedio de $50.2 \pm 9.5 \mathrm{~mm}$ y un diámetro promedio de $7.2 \pm 1.7 \mathrm{~m}$. Las flores fueron hermafroditas, con $254.9 \pm 56.6$ estambres y $915.2 \pm 364$ óvulos (Tabla 1 ). Las flores de Cleistocactus smaragdiflorus tuvieron una forma tubular recta con corola de color rosa y tépalos apicales verdes (Fig. 2D), con un largo de $44.7 \pm 4.1 \mathrm{~mm}$ y un diámetro de $6.9 \pm 1.9 \mathrm{~mm}$. Su polen fue de color amarillo o rosa, y las flores fueron hermafroditas con $230.4 \pm 32.9$ estambres y $1325.6 \pm 303.2$ óvulos (Tabla 1). Una prueba $t$ entre el largo promedio del gineceo y del estambre más largo indició que los estigmas son excertos tanto en $C$. baumannii $(t=5.6, p<0.001)$ como en $C$. smaragdiflorus $(t=6.8, p<0.001)$.

\section{Ciclo floral}


Las flores de Cleistocactus baumannii y C. smaragdiflorus comenzaron a abrirse durante la tarde (16:00 hs) y permanecieron abiertas hasta la mañana del tercer día, alcanzando aproximadamente 48 horas abiertas.

\section{Producción de néctar concentración de azúcares}

La producción total de néctar por flor fue $24.9 \pm 20.2 \mu \mathrm{L}$ en $C$. baumanii y $26.5 \pm$ $21.3 \mu \mathrm{L}$ en $C$. smaragdiflorus, sin diferencias significativas entre ambas especies $(t$ $=-0.2, p=0.9$ ). La mayor proporción del néctar estuvo disponible en el momento de la apertura floral $(79.2 \%$ en $C$. baumannii y $73.5 \%$ en $C$. smaragdiflorus). Para ambas especies, las flores no produjeron más néctar durante el segundo día del ciclo floral. La concentración promedio de néctar fue de $64.5 \pm 4.6 \%$ en $C$. baumanii y $62.2 \pm 3.79 \%$ en $C$. smaragdiflorus, sin diferencias significativas entre las dos especies $(t=1.22, p=0.2384)$.

\section{Sistema reproductivo}

Para ambas especies, solo se produjeron frutos en los tratamientos de polinización natural y de polinización cruzada manual (Fig. 3A). La relación frutos/flores fue significativamente mayor para la polinización cruzada manual que para la polinización natural $(C$. baumannii: $F=-0.7, p<0.0001$; $C$. smaragdiflorus: $F=-$ $0.7, p<0.001$; Fig. $3 A$ ). No se encontraron diferencias significativas entre los valores de la relación semillas/óvulos entre estos dos tratamientos, tanto para $C$. baumannii $(F=-1.7, p=0.1)$ como para $C$. smaragdiflorus $(F=0.6, p=0.7$; Fig. $3 B)$.

La relación frutos/flores para la polinización natural en las tres poblaciones evaluadas de C. baumannii (La Bodeguita: 0.28; poblaciones adicionales: 0.21 y 0.33 ) no fueron significativamente diferentes $\left(X^{2}=0.68, p=0.7133\right)$. Sin embargo, en el caso de $C$. smaragdiflorus, se encontraron diferencias significativas entre poblaciones $\left(X^{2}=15.83, p=0.0004\right)$, con una relación frutos/flores más baja en el sitio La Bodeguita (0.17) comparado con las otras dos poblaciones (0.46 y 0.64 ).

\section{Visitantes florales}

Cleistocactus baumanii fue visitado exclusivamente por picaflores (Trochilidae). En el sitio La Bodeguita, se registraron siete visitas de Chlorostilbon lucidus y dos de Colibri coruscans en 2011, y una visita de cada especie en 2012. Junto con las otras tres poblaciones evaluadas, donde la especie no comparte su hábitat con $C$. 
smaragdiflorus, se registraron nueve visitas de $C$. lucidus, dos de las cuales fueron registradas a través de una videocámara.

Cleistocacus smaragdiflorus fue visitada por Xylocopa sp. (Apidae) y por los picaflores C. lucidus y C. coruscans. En el sitio La Bodeguita, se registraron siete visitas de abejas del género Xylocopa y dos visitas de C. lucidus en 2011; mientras que en 2012, se registró sólo una visita de $C$. lucidus y ninguna visita de abejas. Sólo en una de las cuatro poblaciones adicionales se registraron visitantes florales, que consistieron en una visita de $C$. lucidus y dos visitas de $C$. coruscans. No se registraron visitas a través de las videocámaras.

Para ambas especies de cactáceas, $C$. lucidus introdujo su cabeza casi completamente dentro de las flores, mientras que $C$. coruscans, de mayor tamaño, introdujo su pico y sólo parte de su cabeza. Por su parte, las abejas intentaron acceder al interior de la corola y tocaron el estigma y estambres con su cabeza y abdomen. Los visitantes florales fueron registrados en horarios de la mañana y también durante la tarde. No se encontraron escamas de mariposas nocturnas en los estigmas colectados, y en ninguna de las mariposas nocturnas capturadas en la trampa de luz UV se encontró polen de Cleistocactus en sus cuerpos. No se observaron insectos ladrones de néctar ni tampoco señales de la actividad de los mismos sobre las flores de Cleistocactus.

\section{Modos de especialización a la polinización por aves en especies de cactáceas estudiadas previamente}

La polinización mediada por aves en las flores de cactáceas ha sido documentada en 29 especies incluyendo las dos especies de Cleistocactus (Tabla 2). Esta lista incluye a especies de las subfamilias Opuntioideae (tribu Opuntieae) y Cactoideae (tribus Cereeae, Pachycereeae y Trichocereeae). En todos los casos, las especies fueron visitadas por algún otro gremio de animales además de las aves, a excepción de Cleistocactus baumannii y Denmoza rhodacantha (Capítulo I). Las aves que visitaron las flores de cactáceas incluyeron picaflores (Trochilideae) en todos los casos, a excepción de dos especies de Opuntia de las islas Galápagos (visitadas por pinzones y ruiseñores; Grant y Grant 1981), la cactácea columnar norteamericana Carnegiea gigantea (visitada por diversas especies de aves, pero principalmente palomas; Fleming et al 1996, Fleming et al 2001), y la especie 
columnar barriliforme Echinopsis leucantha (visitada por pinzones; Capítulo I). Además de las aves, el $79 \%$ de las especies fueron visitadas por abejas y el $48 \%$ por animales nocturnos (murciélagos y/o mariposas nocturnas). Un síndrome floral ornitófilo fue reconocido por el/los autor/es en el 59\% de las cactáceas polinizados por aves. Del total de especies estudiadas, el $62 \%$ presentaron flores de color rojizo, y la mayoría de las mismas (90\%) fueron de forma tubular. El análisis de coordenadas principales reveló una diferenciación especial entre las especies con flores rojas y aquellas que presentaron flores blancas o amarillas (Fig. 4).

\section{Especialización fenotípica a las aves en la familia Cactaceae}

Teniendo en cuenta a todas las especies de la familia Cactaceae, se obtuvieron 1517 registros para el color de las flores (incluyendo a todas las especies y a las sub-especies con rasgos florales diferentes), de las cuales 424 fueron rojizas (27\%). Se registró una gran variación en el tamaño floral entre las especies con flores rojizas, desde flores muy pequeñas (por ejemplo, el género Mammillaria), hasta algunas de las flores más grandes de la familia (por ejemplo, Arrojadoa dinae, Cereus stenogonus, Discocactus ackermannii y Hylocereus stenopterus). Una prueba de aleatorización indicó que el largo de la corola (medio y mediano) de las flores rojizas no fue significativamente diferente al de todas las especies. Contrariamente, el diámetro de la corola de las flores rojizas (medio y mediano) fue significativamente menor para las flores rojizas que para el resto de las especies con flores de otros colores.

Con respecto al ciclo floral, se obtuvo información para 621 entidades (especies o subspecies con diferencias), de las cuales el 66\% mostró un tiempo de antesis diurno. Dentro de este grupo, las flores rojizas estuvieron representadas por 139 especies, con una proporción de flores de antesis diurna significativamente mayor (93\%) que la de todas las especies evaluadas $(p<$ $0.001)$.

El total de especies para las cuales se obtuvo información acerca de la forma floral fueron 1137 , de las cuales el 7,92\% mostraron flores tubulares. Dentro de las especies con flores rojizas, la proporción de flores tubulares $(20.1 \%)$ fue significativamente mayor que al compararlas con todas las especies evaluadas ( $p$ $<0.001)$. Un resultado similar se obtuvo al comparar la proporción de flores con 
forma parcialmente tubular (tubular-campanuliforme, tubular-embudo, etc.) en la familia entera $(20.1 \%)$ con la proporción dentro de las especies con flores rojizas (29.2\%). Además, la proporción de flores rojizas entre las especies con flores de forma tubular $(71.1 \%)$ o parcialmente tubular $(40.6 \%)$ fue significativamente mayor que lo esperado por azar $(p<0.001)$.

Para todas las subfamilias, a excepción de Maihuenioideae, se registraron especies con flores rojizas. La subfamilia con la mayor cantidad de especies con flores rojizas fue Pereskioideae (Fig. 5). Dentro de la subfamilia Opuntioideae, la proporción más alta de especies con flores rojizas se encontró en la tribu Austrocylindropuntieae, seguida por Opuntieae y Cylindropuntieae. En la subfamilia Cactoideae, la mayor proporción de especies de flores rojizas se registró en la tribu Pachycereeae, seguida por Cacteae, Cereeae y Trichocereeae. Las pruebas de aleatorización indicaron que para la mayoría de las subfamilias y tribus, la proporción de especies con flores rojizas no fue significativamente mayor a lo esperado por azar (Fig. 5). Para esto, la proporción de flores rojizas fue calculada para las subfamilias y para toda la familia, obteniendo resultados similares para todos los casos. En resumen, 65 géneros de cactáceas $(52.4 \%$ del total de la familia) mostraron al menos una especie con flores rojizas, y 12 géneros mostraron una proporción de especies con flores rojizas mayor al $70 \%$ :

Castellanosia, Cleistocactus, Denmoza, Disocactus, Geohintonia, Matucana, Melocactus, Neolloydia, Nopalea, Oreocereus, Pelecyphora y Schlumbergera.

\section{DISCUSIÓN}

\section{Biología reproductiva de Cleistocactus}

Ambas especies de Cleistocactus estudiadas presentaron flores rojas, tubulares, con estigma excerto y polen coloreado. Entre los rasgos florales señalados como típicos de flores de aves, el color rojo es considerado como la característica que define a las flores ornitófilas más acertadamente (Thomson et al. 2000, Wilson et al. 2004, Curti y Ortega-Baes 2011). Particularmente, éste es un rasgo recurrente entre las cactáceas con aves como polinizadores. Por otro lado, la forma tubular es también un rasgo citado como ornitófilo (Faegri y van der Pijl 1979) que podría operar como una barrera física a algunos otros polinizadores (Brown y KodricBrown 1979). En el caso de las especies estudiadas en este Capítulo, la forma 
tubular angosta de las flores podría restringir el acceso al néctar por parte de otros animales además de los picaflores. En la familia Cactaceae, las flores rojizas tubulares han sido citadas para varias especies polinizadas por aves (Locatelli y Machado 1999, Scobell y Scott 2002, Nassar y Ramírez 2004, Colaço et al. 2006, Nassar et al. 2007, Fagua y Ackerman 2011). Estos rasgos también están presentes entre otras cactáceas de la tribu Trichocereeae, tales como Denmoza rhodacantha y Oreocereus celsianus, cuyos polinizadores principales son picaflores (Larrea Alcázar y López 2011, Capítulo I). El color del polen de las especies estudiadas apoya la idea presentada por Rose y Barthlott (1994), que sugiere que el polen de color rojo es un rasgo ornitófilo en las cactáceas; sin embargo, algunas especies polinizadas por aves tienen polen amarillo, tal como se reportó aquí para algunos individuos de $C$. smaragdiflorus.

La cantidad de néctar producido por flor fue similar para ambas especies, presentando un valor intermedio entre otras especies de cactáceas polinizadas por aves. Sin embargo, la concentración de azúcares en el néctar de $C$. baumannii y $C$. smaragdiflorus fue las más alta entre las cactáceas polinizadas por aves. Esto contrasta con las concentraciones diluidas que han sido registradas para flores de aves en general (Pyke y Waser 1981, Scogin 1985, Stiles y Freeman 1993, Nicolson y Fleming 2003, Fenster et al. 2006, Curti y Ortega-Baes 2011); sin embargo, este hallazgo no es la única excepción registrada (Parra et al. 1993, Nassar y Ramirez 2004). La producción de néctar fue menor y la concentración de azúcares mayor (más del doble) que los valores registrados por Scogin (1985) para estas mismas especies; sin embargo, los valores aquí presentados fueron registrados en poblaciones naturales. Esta diferencia podría ser una consecuencia de las condiciones climáticas de la región del Chaco Seco durante el período reproductivo.

El ciclo floral se extendió durante más de un día en ambas especies, un rasgo poco común en la familia Cactaceae, ya que la mayoría de las especies muestran flores de un día (Fleming et al. 1996, 2001, Ortega-Baes et al. 2011, Alonso-Pedano y Ortega-Baes 2012, Ortega-Baes y Gorostiague 2013, Capítulo I). Un ciclo floral extendido podría ser considerado una estrategia para disminuir la competencia por polinizadores (Nassar et al. 2007) o para incrementar las 
oportunidades de recibir visitas de vectores de polen para hacer frente a su escasez o baja frecuencia (Primack 1985, Torres-Díaz et al. 2011).

Las especies estudiadas fueron auto-incompatibles; por lo tanto, dependen exclusivamente de sus polinizadores para la producción de frutos y semillas. Esta característica es común entre las especies de cactáceas de la tribu Trichocereeae (Capítulo 1) y para las cactáceas columnares en general (Mandujano et al. 2010); sin embargo, se han registrado algunas especies con auto-compatibilidad para esta tribu (Sahley 1996, Walter 2010, Larrea-Alcázar y López 2011). A pesar de la dependencia de los polinizadores, los resultados revelaron una baja tasa de visita para ambas especies. Las flores de C. baumannii fueron visitadas por dos especies de picaflores, mientras que las flores de $C$. smaragdiflorus fueron visitadas por dos especies de picaflores y también por abejas. Para otras especies de cactáceas ornitófilas con ciclos florales extendidos, se han registrado visitas de mariposas nocturnas (por ejemplo, Oreocereus celcianus; Larrea Alcázar y López 2011); sin embargo, no se encontró evidencia de visitas nocturnas a las flores de las especies aquí estudiadas, aun cuando las flores permanecieron abiertas durante la noche.

La baja tasa de visitas de los polinizadores se reflejó en un bajo valor de la relación frutos/flores en el tratamiento de polinización natural: menos del $30 \%$ en C. baumannii y $20 \%$ en C. smaragdiflorus en el sitio La Bodeguita. Estos valores fueron similares en las otras poblaciones evaluadas de $C$. baumannii. Sin embargo, en las poblaciones restantes de esta especie, la polinización natural incrementó significativamente los valores de la relación frutos/flores. Este último hallazgo podría ser consecuencia de varios factores que no fueron evaluados en este estudio, tales como la variación en la abundancia de los polinizadores o en el comportamiento demográfico de las diferentes poblaciones (por ejemplo, tamaño de los individuos y producción de flores). Los valores de la relación frutos/flores para la polinización natural fueron menores que los obtenidos para la polinización cruzada manual, sugiriendo que la producción de frutos está limitada por polen (polinizadores) en ambas especies. Sumada a la baja tasa de visitas florales, otra posible causa de la baja producción de frutos podría estar relacionada con el comportamiento de los visitantes y la auto-incompatibilidad de las cactáceas. Los 
picaflores típicamente visitan varias flores de la misma planta, depositando polen autógamo en el estigma. Adicionalmente, dado que ambas especies presentan reproducción clonal, también es posible que el polen recibido de flores de individuos vecinos fuera autógamo (es decir, de clones idénticos). Este hallazgo ha sido demostrado recientemente para Echinopsis thelegona, una especie de cactácea clonal que habita en el mismo área de estudio y cuya reproducción sexual es extremadamente rara (Ortega-Baes y Gorostiague 2013).

De acuerdo al enfoque propuesto por Ollerton et al. (2007), ambas especies de Cleistocactus mostraron una especialización fenotípica, ya que sus flores mostraron rasgos típicamente ornitófilos. Sin embargo, solo C. baumanii parece ser funcionalmente especializado (aunque un estudio a lo largo de un tiempo mayor y sobre un área mayor podría revelar otros polinizadores). Contrariamente, $C$. smaragdiflorus presentó generalización funcional porque sus flores fueron visitadas por más de un gremio de polinizadores. Por otro lado, el sistema de polinización de ambas especies fue ecológicamente generalizado. El presente estudio constituye el primer reporte detallado sobre la ecología reproductiva de especies del género Cleistocactus. Notoriamente, no apoya la hipótesis inicial de que el género presenta sistemas de polinización especializados (Anderson 2001, Hunt 2006); al menos no para los múltiples modos en los que una especie puede ser considerada especializada (Ollerton et al. 2007).

\section{Modos de especialización a las aves en especies de cactáceas estudiadas previamente}

En la familia Cactaceae, la polinización mediante aves ha sido registrada en 29 especies que se distribuyen a lo largo de todo el hemisferio occidental. Estas especies incluyen a representantes de las subfamilias Opuntioideae y Cactoideae. Si bien una gran proporción de estas especies se concentran en ambientes tropicales, no es adecuado manifestar que este modo de polinización esté asociado un componente geográfico, debido a que existe un sesgo de muestreo generado por la alta riqueza de especies en los trópicos (Ollerton y Cranmer 2002). Esta interacción planta-animal parece ocurrir a lo largo de todo el rango geográfico de la familia, y estaría limitada primariamente por la distribución geográfica de los polinizadores más que por las especies de cactáceas en sí. Las flores con corolas 
rojizas estuvieron presentes en el $62 \%$ de las especies estudiadas que fueron visitadas por aves. Teniendo en cuenta este rasgo floral, también se podría diferenciar a las especies fenotípicamente especializadas a las aves en base al análisis de coordenadas principales.

La concentración de azúcares en el néctar producido por las cactáceas polinizados por aves fue bastante uniforme y se correspondió con lo indicado para flores de aves (Pyke y Waser 1981, Stiles y Freeman 1993). No obstante, la cantidad de néctar producido por flor fue altamente variable. Las especies con flores de tamaño mayor produjeron una mayor cantidad de néctar que las especies con flores más pequeñas. Esto sugiere que, al menos para las cactáceas, la producción de néctar se correlaciona con el tamaño floral más que con la especialización fenotípica. Este hallazgo fue registrado también en otros sistemas con polinización por aves (Geerts y Pauw 2009).

Sólo dos especies de cactáceas (Cleistocactus baumannii y Denmoza rhodacantha) fueron visitadas exclusivamente por aves, siendo por lo tanto funcionalmente especializadas. El resto de las especies fueron visitadas además por otros grupos de animales, que incluyeron principalmente abejas, murciélagos y mariposas nocturnas. A pesar de presentar flores ornitófilas, los sistemas de polinización estudiados fueron generalistas, aunque habían sido clasificados como especializados a la polinización por aves por el/los autor/es original/es. Este patrón sugiere que, en general, los rasgos florales ornitófilos no restringen a otros polinizadores, además de las aves, que podrían utilizar los recursos ofrecidos por las flores. Sin embargo, sería necesario evaluar que tanto aportan estos polinizadores adicionales a la reproducción de las plantas (Rosas-Gerrero et al. 2014, Ashworth et al. 2015).

\section{Especialización fenotípica a las aves en la familia Cactaceae}

Dentro de la familia Cactaceae, la especialización a las aves (teniendo en cuenta solo el rasgo del color rojizo en la corola) estuvo presente en 386 especies. Este número representa aproximadamente el $27 \%$ de las especies de la familia e incluye a representantes de casi todas las subfamilias y tribus. Además, hay 12 géneros de cactáceas que podrían ser considerados como fenotípicamente especializados a las aves, dado que la mayoría de sus especies producen flores 
rojizas: Castellanosia, Cleistocactus, Denmoza, Disocactus, Geohintonia, Matucana, Melocactus, Neolloydia, Nopalea, Oreocereus, Pelecyphora y Schlumbergera. Muchos de estos géneros ya han sido citados como ejemplos de especialización a las aves en la literatura taxonómica (Rose y Barthlott 1994, Anderson 2001, Pimienta-Barrios y del Castillo 2002, Hunt 2006). Sin embargo, los resultados podrían sobreestimar el número de especies con una especialización fenotípica a la polinización por aves real, debido a que algunas serían polinizadas principalmente por abejas. Este podría ser el caso de los géneros Geohintonia, Mammillaria, Neolloydia, Pelecyphora, Pereskia, entre otros (Grant y Grant 1978, Anderson 2001, Hunt 2006, Valverde et al. 2015).

Los resultados, basados en la información disponible para toda la familia, indicaron que entre las cactáceas, las flores rojizas estuvieron asociadas a la antesis diurna y a la forma tubular de la corola. Además, si bien existe una gran variación en cuanto al tamaño floral entre las especies de cactáceas con flores rojizas, los resultados mostraron que el diámetro floral fue menor en este conjunto de especies comparado con el resto de la familia. Esta tendencia también apoya la idea de que la forma tubular es más común entre las especies de cactáceas con flores rojizas. La ausencia de una relación entre el tamaño (longitud) de las flores y el color rojizo de la corola podría indicar que no existen restricciones en el tamaño de las flores que son polinizadas por aves. Las especies de cactáceas visitadas por aves incluyeron flores de tamaño muy pequeño (por ejemplo, Melocactus y Micranthocereus) y flores de gran tamaño (por ejemplo, Echinopsis atacamensis). En cambio, el ancho de la corola podría restringir el acceso al néctar por parte de animales que no pueden ingresar en las flores angostas, tal como se ha descripto para las especies de Cleistocactus estudiadas. Para otros grupos de plantas, se ha demostrado que las dimensiones de las flores y los picos de los picaflores no están tan fuertemente asociados como se esperaría; lo cual podría ser atribuido a otros factores asociados a las características del néctar o a interacciones con otras especies (Temeles et al. 2002, pero ver Geerts y Pauw 2009).

Finalmente, se puede manifestar que la especialización fenotípica a la polinización por aves habría evolucionado varias veces en la familia Cactaceae, dado que las flores rojizas son un rasgo recurrente a lo largo de su filogenia. Esta 
especialización solo se ha registrado al nivel de géneros (por ejemplo, Cleistocactus, Melocactus, y Oreocereus) pero no para taxa superiores. Es posible que para algunos casos, este rasgo haya evolucionado a partir de ancestros con flores polinizadas por abejas (Pimienta-Barrios y del Castillo 2002), tal como ha sido sugerido para otras familias de plantas (Alcantara y Lohmann 2010, van der Niet y Johnson 2012, Ashworth et al. 2015).

\section{Conclusiones}

Este estudio es el primero en proveer una mejor comprensión de la especialización a las aves en la familia Cactaceae. Así como en otras familias de angiospermas, el síndrome floral ornitófilo, concebido como una especialización fenotípica, puede ser reconocido en las cactáceas. Sin embargo, no se puede declarar que exista una correspondencia entre esta especialización fenotípica y los verdaderos polinizadores que visitan y polinizan efectivamente a las flores de aves (Capítulo I). El hecho de atribuirle el servicio de polinización a un polinizador (o gremio de polinizadores) exclusivo no siempre es acertado debido a que las observaciones de campo pueden mostrar diferentes interacciones con otras especies. Además, la especialización fenotípica no necesariamente provee de ventajas para reproducción, debido a que un polinizador oportunista podría llegar a ser tan efectivo como el polinizador teóricamente especializado (Nassar y Ramirez 2004, Ollerton et al. 2007, Frick et al. 2013). Sumado a esto, los casos de especialización funcional en los sistemas de polinización son escasos, y la variación en los polinizadores entre poblaciones de una misma especie de planta es notable. Esto apoya la idea de que la especialización no es determinada exclusivamente por los propios rasgos de las plantas, sino también por las condiciones ecológicas del ambiente en el que habitan las especies (Ollerton et al. 2007). La evidencia actual apoya el hecho de que la especialización funcional y ecológica a la polinización por aves en la familia Cactaceae es rara. Tal como indican los resultados presentados, calificar a un sistema de polinización como especializado debe hacerse con cautela cuando el modo en el que éste es especializado (o generalizado; sensu Ollerton et al. 2007) no está claramente definido. Las cactáceas son comúnmente definidas como especializadas a ciertos tipos de polinizadores; sin embargo, esta determinación generalmente está 
basada en los rasgos florales (el concepto de síndrome floral) o en el principio del polinizador más efectivo (sensu Stebbins 1970). Debido a la gran diversidad de esta familia, aún permanecen muchas especies sin información disponible sobre sus interacciones ecológicas. Por lo tanto, es esencial realizar estudios adicionales para poder entender las interacciones entre las cactáceas y sus mutualistas.

\section{REFERENCIAS BIBLIOGRÁFICAS}

Aizen M.A., Feinsinger P. (1994) Forest fragmentation, pollination, and plant reproduction in a Chaco dry forest, Argentina. Ecology 75: 330-351.

Alcantara S., Lohmann L.G. (2010) Evolution of floral morphology and pollination system in Bignonieae (Bignoniaceae). American Journal of Botany 97: 782796.

Alonso-Pedano M., Ortega-Baes P. (2012) Generalized and complementary pollination system in the Andean cactus Echinopsis schickendantzii. Plant Systematics and Evolution 298: 1671-1677.

Anderson E.F. (2001) The Cactus Family. Timber Press, Oregon, USA: 776 pp. Ashworth L., Aguilar R., Martén-Rodríguez S., Lopezaraiza-Mikel M., Avila-Sakar G., Rosas-Guerrero V., Quesada M. (2015) Pollination syndromes: a global pattern of convergent evolution driven by the most effective pollinator. En: Pontarotti P. (ed.) Evolutionary biology: biodiversification from genotype to phenotype. Springer International Publishing: 203-224.

Bárcenas R.T., Yesson C., Hawkins J.A. (2011) Molecular systematics of the Cactaceae. Cladistics 27: 470-489.

Bianchi A.R. (1996) Temperaturas medias estimadas para la región noroeste de Argentina. INTA, Salta, Argentina.

Bianchi A.R., Yañez C.E. (1992). Las precipitaciones en el noroeste argentino. INTA, Salta, Argentina.

Brown J.H., Kodric-Brown A. (1979) Convergence, competition, and mimicry in a temperate community of hummingbird-pollinated flowers. Ecology 60: 10221035.

Bustamante E., Casas A., Búrquez A. (2010) Geographic variation in reproductive success of Stenocereus thurberi (Cactaceae): Effects of pollination timing and pollinator guild. American Jounal of Botany 97: 2020-2030. 
Colaço M.A.S., Fonseca R.B.S., Lambert S.M., Costa C.B.N., Machado C.G., Borba E.L. (2006) Biologia reprodutiva de Melocactus glaucescens Buining \& Brederoo e M. paucispinus G. Heimen \& R. Paul (Cactaceae), na Chapada Diamantina, Nordeste do Brasil. Revista Brasileira de Botánica 29: 239-249.

Curti R., Ortega-Baes P. (2011) Relationship between floral traits and floral visitors in two coexisting Tecoma species (Bignoniaceae). Plant Systematics and Evolution 293: 207-211.

Dar S., Arizmendi M., Valiente-Banuet A. (2006) Diurnal and Nocturnal Pollination of Marginatocereus marginatus (Pachycereeae: Cactaceae) in Central Mexico. Annals of Botany 97: 423-427.

de Viana M.L., Ortega-Baes P., Saravia M., Badano E., Schlumpberger B. (2001) Biología floral y polinizadores de Trichocereus pasacana (Cactaceae) en el Parque Nacional Los Cardones, Argentina. Revista de Biología Tropical 49: 279-285.

Di Rienzo J.A., Casanoves F., Balzarini M.G., Gonzalez L., Tablada M., Robledo C.W. (2009) InfoStat versión 2009. Argentina: Grupo InfoStat, FCA, Universidad Nacional de Córdoba.

Díaz L., Cocucci A.A. (2003) Functional Gynodioecy in Opuntia quimilo (Cactaceae), a Tree Cactus Pollinated by Bees and Hummingbirds. Plant Biology 5: 531-539.

Faegri K., van der Pijl L. (1979) The principles of pollination ecology. London: Pergamon Press: 248 pp.

Fagua J.C., Ackerman J.D. (2011) Consequences of floral visits by ants and invasive honeybees to the hummingbird-pollinated, Caribbean cactus Melocactus intortus. Plant Species Biology 26: 193-204.

Fenster C.B., Armbruster W.S., Dudash M.R., Wilson P., Thomson J.D. (2004) Pollination syndromes and floral specialization. Annual Review of Ecology and Systematics 35: 375-103.

Fenster C.B., Cheely G., Dudash M.R., Reynolds R.J. (2006) Nectar reward and advertisement in hummingbirds-pollinated Silene virginica (Caryophillaceae) American Journal of Botany 93: 1800-1807. 
Fleming T.H., Holland J.N. (1998) The evolution of obligate pollination mutualisms: senita cactus and senita moth. Oecologia 114: 368-375.

Fleming T.H., Shaley C.T., Holland J.N., Nason J.D., Hamrick J.L. (2001) Sonoran desert columnar cacti and the evolution of generalized pollination systems. Ecological Monographs 71: 511-530.

Fleming T.H., Tuttle M.D., Horner M.A. (1996) Pollination biology and the relative importance of nocturnal and diurnal pollinators in three species of Sonoran desert columnar cacti. The Southwestern Naturalist 41: 257-269.

Frick W.R., Price R.D., Heady III P.A., Kay K.M. (2013) Insectivorous Bat Pollinates Columnar Cactus More Effectively per Visit than Specialized Nectar Bat. The American Naturalist 181: 137-144.

Geerts S., Pauw A. (2009) Hyper-specialization for long-billed bird pollination in a guild of South African plants: the Malachite Sunbird pollination syndrome. South African Journal of Botany, 75: 699-706.

Gibson A.C., Nobel P.S. (1986) The cactus primer. Harvard University Press, Cambridge, USA: $286 \mathrm{pp}$.

Gómez J.M., Zamora R. (1999) Generalization vs. specialization in the pollination system of Hormatophylla spinosa (cruciferae). Ecology 80: 796-805.

Grant V., Grant K.A. (1979) The pollination spectrum in the southwestern American cactus flora. Plant Systematics and Evolution 133: 29-37.

Grant B.R., Grant P.R. (1981) Exploitation of Opuntia cactus by Birds on the Galápagos. Oecologia 49: 179-187.

Hegland S.J., Totland O. (2005) Relationships between species floral traits and pollinator visitation in a temperate grassland. Oecologia 145: 586-594.

Heredia J. (2011) Picaflores de Argentina. L.O.L.A, Buenos Aires, Argentina: 104 pp.

Hunt D. (2006) The new cactus lexicon. DH Books, Milborne Port, England: 373, $526 \mathrm{pp}$.

Ibarra-Cerdeña C., Iñiguez-Dávalos L., Sánchez-Cordero V. (2005) Pollination ecology of Stenocereus queretaroensis (Cactaceae) a chiropterophilous columnar cactus, in a tropical dry forest of Mexico. American Journal of Botany 92: 503-509. 
Larrea-Alcázar D.M., López R.P. (2011) Pollination biology of Oreocereus celsianus (Cactaceae), a columnar cactus inhabiting the high subtropical Andes. Plant Systematics and Evolution 295: 129-137.

Locatelli E., Machado I.C. (1999) Comparative study of the floral biology in two ornithophilous species of Cactaceae: Melocactus zehntneri and Opuntia palmadora. Bradleya 17: 75-85.

Mandujano M.C., Carrillo-Angeles I., Martínez-Peralta C., Golubov J. (2010) Reproductive biology of Cactaceae. En: Ramawat K.J. (ed) Desert Plants. Springer-Verlag, Berlin, Germany: 157-171.

Mc Gregor S.E., Alcorn S.M., Olin G. (1962) Pollination and Pollinating Agents of the Saguaro. Ecology 43: 259-267.

Narosky T., Yzurieta D. (2010) Guía para la identificación de las aves de Argentina y Uruguay. Vazquez Mazzini Editores, Buenos Aires, Argentina: 427 pp.

Nassar J.M., Ramírez N., Lampo M., González J.A., Casado R., Nava F. (2007) Reproductive biology and mating system estimates of two andean melocacti, Melocactus schatzlii and $M$. andinus (Cactaceae). Annals of Botany 99: 29-38.

Nassar J.M., Ramírez N. (2004) Reproductive biology of the melon cactus, Melocactus curvispinus (Cactaceae). Plant Systematics and Evolution 248: 31-44.

Nicolson S.W., Fleming P.A. (2003) Nectar as food for birds: the physiological consequences of drinking dilute sugar solutions. Plant Systematics and Evolution 238: 139-153.

Ollerton J. (1996) Reconciling ecological processes with phylogenetic patterns: The apparent paradox of plant-pollinator systems. Journal of Ecology 84: 767-769.

Ollerton J., Alarcón R., Waser N.M., Price M.V., Watts S., Cranmer L., Hingston A., Peter C.I., Rotenberry J. (2009) A global test of the pollination syndrome hypothesis. Annals of Botany 103: 1471-1480.

Ollerton J., Cranmer L. (2002) Latitudinal trends in plant-pollinator interactions: are tropical plants more specialized? Oikos 98: 340-350. 
Ollerton J., Killick A., Lamborn E., Watts S., Whiston M. (2007) Multiple meanings and modes: on the many ways to be a generalist flower. Taxon 53: 717-728.

Ordones Rego J., Franceschinelli E.V., Zappi D.C. (2012) Reproductive biology of a highly endemic species: Cipocereus laniflorus N.P. Taylor \& Zappi (Cactaceae). Acta Botanica Brasilica 26: 243-250.

Ortega-Baes P., Aparicio-González M., Galíndez G. (2010) Vivipary in the cactus family: An evaluation of 25 species from northwestern Argentina. Journal of Arid Environments 74: 1359-1361.

Ortega-Baes P., Gorostiague P. (2013) Extremely reduced sexual reproduction in the clonal cactus Echinopsis thelegona. Plant Systematics and Evolution 299: 785-791.

Ortega-Baes P., Saravia M., Suhring S., Godínez-Álvarez H., Zamar M. (2011) Reproductive biology of Echinopsis terschekii (Cactaceae): the role of nocturnal and diurnal pollinators. Plant Biology 13: 33-40.

Parra V., Vargas C.F., Eguiarte L.E. (1993) Reproductive biology, pollen and seed dispersal, and neighborhood size in the hummingbird-pollinated Echeveria gibbiflora (Crassulaceae). American Journal of Botany 80: 153-159.

Phillips S.J., Anderson R., Schapire R.E. (2006) Maximum entropy modeling of species geographic distributions. Ecological Modelling 190: 231-259.

Pimienta-Barrios E., del Castillo R.F. (2002) Reproductive biology. En: Nobel P.S. (ed.) Cacti: biology and uses. University of California Press, Los Angeles, USA: 163-183.

Primack R.B. (1985) Longevity of individual flowers. Annual Review of Ecology and Systematics 16: 15-37.

Pyke G.H., Waser N.M. (1981) The production of dilute nectar by hummingbird and honeyeater flowers. Biotropica 13: 260-270.

R Development Core Team. 2012. R: A language and environment for statistical computing. R Foundation for Statistical Computing, Vienna, Austria: URL http://www.R-project.org/

Rosas-Guerrero V., Aguilar, R., Martén-Rodríguez S., Ashworth L., LopezaraizaMikel M., Bastida J.M., Quesada M. (2014) A quantitative review of pollination syndromes: do floral traits predict effective pollinators? Ecology 
Letters 17: 388-400.

Rose M.J., Barthlott W. (1994) Coloured pollen in Cactaceae: a mimetic adaptation to hummingbird-polination? Botanica Acta 107: 402-406.

Sahley C.T. (1996) Bat and hummingbird pollination of an autotetraploid columnar cactus, Weberbauerocereus weberbaueri (Cactaceae). American Journal of Botany 83: 1329-1336.

Saleme Aona L.Y., Machado M., Pansarin E.R., Cardoso de Castro C., Zappi D., do Amaral M.C. (2006) Pollination biology of three Brazilian species of Micranthocereus Backeb. (Cereeae, Cactoideae) endemic to the "campos rupestres". Bradleya 24: 39-52.

Schlumpberger B.O., Badano E. (2005) Diversity of floral visitors to Echinopsis atacamensis subsp. pasacana (Cactaceae). Haseltonia 11: 18-26.

Scobell S.A., Scott P.E. (2002) Visitors and Floral Traits of a HummingbirdAdapted Cactus (Echinocereus Coccineus) Show Only Minor Variation along an Elevational Gradient. American Midland Naturalist 147:1-15.

Scogin R. (1985) Nectar constitutens of the Cactaceae. The Southwestern Naturalist 30: 77-82.

Stebbins G.L. (1970) Adaptive radiation of reproductive characteristics in angiosperms I: Pollination mechanisms. Annual Review of Ecology and Systematics 1: 307-26.

Stiles F.G., Freeman C.E. (1993) Patterns in floral nectar characteristics of some bird-visited plant species from Costa Rica. Biotropica 25: 191-205.

Temeles E.J., Linhart Y.B., Masonjones M., Masonjones H.D. (2002) The role of flower width in hummingbird bill length-flower length relationships. Biotropica 34: 68-80.

Thomson J.D., Wilson P., Valenzuela M. (2000) Pollen presentation and pollination syndromes, with special reference to Penstemon. Plant Species Biology 15: $11-29$.

Torres-Díaz C.S., Gómez-González S., Stotz G.C., Torres-Morales P., Paredes B., Pérez-Millaqueo M., Gianoli E. (2011) Extremely long-lived stigmas allow extended cross-pollination opportunities in a high Andean plant. PloS one 6: e19497. 
Valiente-Banuet A., Rojas-Martínez A., Arizmendi M.C., Da' Vila P. (1997) Pollination Biology Of Two Columnar Cacti (Neobuxbaumia Mezcalaensis And Neobuxbaumia Macrocephala) In The Tehuacan Valley, Central Mexico. American Journal of Botany 84: 452-455.

Valverde P.L., Jiménez-Sierra C., López-Ortega G., Zavala-Hurtado J.A., RivasArancibia S., Rendón-Aguilar B., Pérez-Herández M.A., Cornejo-Romero A., Carrillo-Ruiz H. (2015) Floral morphometry, anthesis, and pollination success of Mammillaria pectinifera (Cactaceae), a rare and threatened endemic species of Central Mexico. Journal of Arid Environments 116: 2932.

van der Niet T., Johnson S.D. (2012) Phylogenetic evidence for pollinator-driven diversification of angiosperms. Trends in Ecology \& Evolution 27: 353-361.

Waser N.M. (2006) Specialization and generalization in pant-pollinator interactions: a historical perspective. En: Waser N.M., Ollerton J. (eds.) Plant-pollinator interactions: from specialization to generalization. The University of Chicago Press, Chicago, USA: $445 \mathrm{pp}$.

Waser N.M., Chittka L., Price M.V., Williams N.M., Ollerton J. (1996) Generalization in pollination systems and why it matters. Ecology 77: 104360

Wilson P., Castellanos M.C., Houge J.N., Thomson J.D., Armbruster S.W. (2004) A multivariate search for pollination syndromes among penstemons. Oikos 104: 346-361. 
Tabla 1. Características florales (media, desvío estándar, mínimo y máximo) de Cleistocactus baumannii $(\mathrm{n}=20)$ y $C$. smaragdiflorus $(n=33)$. Aquellas medidas que varían significativamente entre especies $(p<0.05)$ están indicadas con asterisco $\left.{ }^{*}\right)$. DE: Desvío estándar. Mín: Mínimo, Máx: Máximo.

\begin{tabular}{lrrrrrrrrr}
\hline & \multicolumn{3}{c}{ Cleistocactus baumanii } & \multicolumn{3}{c}{ Cleistocactus smaragdiflorus } & \multicolumn{2}{c}{ Prueba T } \\
\cline { 2 - 9 } & Media & DE & Mín & Máx & Media & DE & Mín & Máx \\
\cline { 2 - 9 } Diámetro de la corola & 7.19 & 1.68 & 3.39 & 9.32 & 6.97 & 1.89 & 3.18 & 10.15 & 0.43 \\
Longitud total & 50.2 & 9.53 & 28.85 & 62.19 & 44.71 & 4.09 & 34.01 & 52.47 & $2.44^{*}$ \\
Longitud del gineceo & 41.28 & 11.05 & 19.62 & 55.79 & 35.1 & 4.9 & 18.75 & 43.54 & $2.36^{*}$ \\
Longitud del estilo & 38.61 & 10.53 & 18.03 & 52.38 & 32.57 & 4.71 & 15.89 & 40.09 & $2.42^{*}$ \\
Longitud del estigma & 2.67 & 0.89 & 1.13 & 4.13 & 2.66 & 0.73 & 0.96 & 4.42 & 0.04 \\
Diámetro del estigma & 3.16 & 0.75 & 2.01 & 4.29 & 3.8 & 1.2 & 1.69 & 6.73 & $-2.40^{*}$ \\
Longitud del estambre más largo & 35.81 & 8.35 & 18.32 & 45.43 & 31.43 & 4.44 & 19.57 & 38.94 & $2.16^{*}$ \\
Longitud del estambre más corto & 27.27 & 7.42 & 7.38 & 37.15 & 25.93 & 6.27 & 9.95 & 36.89 & 0.71 \\
Longitud del ovario & 8.39 & 1.26 & 6 & 10.59 & 9.58 & 1.4 & 6.69 & 12.95 & $-3.10^{*}$ \\
Diámetro del ovario & 6.87 & 0.99 & 5.13 & 8.21 & 7.63 & 0.88 & 5.67 & 9.43 & $-2.91^{*}$ \\
Longitud interna del ovario & 5.02 & 1.4 & 1.97 & 7.39 & 5.96 & 1.24 & 3.56 & 9.49 & $-2.55^{*}$ \\
Diámetro interno del ovario & 3.62 & 0.97 & 2.11 & 4.92 & 3.85 & 0.82 & 2.33 & 5.93 & -0.9 \\
Largo cámara nectarial & 7.39 & 2.93 & 1.71 & 11.13 & 4.33 & 1.16 & 2.39 & 6.52 & $4.45^{*}$ \\
Ancho cámara nectarial & 3.92 & 1.15 & 1.5 & 5.57 & 3.24 & 0.86 & 1.78 & 5.43 & $2.45^{*}$ \\
\hline
\end{tabular}




\begin{tabular}{lrrrrrrrrr}
\hline Número de estambres & 254.90 & 56.56 & 151 & 380 & 230.42 & 32.91 & 143 & 283 & 1.76 \\
Número de lóbulos & 7.35 & 0.59 & 6 & 8 & 7.55 & 1.23 & 6 & 11 & -0.78 \\
Número de óvulos & 915.15 & 364.01 & 200 & 1800 & 1325.64 & 303.17 & 772 & 2027 & $-4.43^{*}$ \\
\hline
\end{tabular}


Tabla 2. Rasgos florales y polinizadores de 29 especies de cactáceas en las que se ha registrado polinización por aves. Forma floral: Ta: tazón; E: embudo; Tu: tubular. Color: M: magenta; P: rosa; R: rojo; B: blanco; A: amarillo. Síndrome floral propuesto por el(los) autor(es) original(es): Q: quiropteófilo; O: ornitófilo; M: melitófilo. Ciclo floral: D: antesis diurna; N: antesis nocturna; ext.: ciclo extendido a la mañana siguiente; 24 h+: ciclo dura más de un día completo. Visitantes florales: Pi: picaflores; Mu: murciélagos; Ab: abejas; Ma: mariposas nocturnas. Los nombre científicos corresponden a aquellos utilizados por el(los) autor(es) original(es). *

Valores registrados para la flor hermafrodita.

\begin{tabular}{|c|c|c|c|c|c|c|c|c|c|c|c|}
\hline Epecie & Tribu & $\begin{array}{l}\text { Forma } \\
\text { floral }\end{array}$ & Color & $\begin{array}{l}\text { Longitud } \\
\text { corola } \\
\text { (media } \pm \text { SD, } \\
\mathrm{mm} \text { ) }\end{array}$ & $\begin{array}{l}\text { Diámetro } \\
\text { corola } \\
\text { (media } \pm \\
\text { SD, mm) }\end{array}$ & $\begin{array}{l}\text { Producción } \\
\text { promedio de } \\
\text { néctar/flor } \\
\text { (media } \pm \text { SD, } \mu \mathrm{l} \text { ) }\end{array}$ & $\begin{array}{l}\text { Concentración } \\
\text { promedio de } \\
\text { azúcares } \\
\text { (media } \pm \text { SD, } \\
\% \text { ) }\end{array}$ & $\begin{array}{l}\text { Síndrome } \\
\text { floral }\end{array}$ & Antesis & $\begin{array}{l}\text { Visitantes } \\
\text { florales }\end{array}$ & Referencia \\
\hline Carnegiea gigantea & Pachycereae & $\mathrm{E}$ & $\mathrm{B}$ & $112.2 \pm 3.2$ & $24.9 \pm 0.9$ & $1070 \pm 90$ & $24-25$ & Q & $\mathrm{N}$, ext. & $\begin{array}{l}\mathrm{Pi}, \mathrm{Mu}, \mathrm{Ab}, \mathrm{Ma} \text {, } \\
\text { palomas }\end{array}$ & $\begin{array}{l}\text { Fleming et al. 1996; } \\
\text { Fleming et al. } 2001\end{array}$ \\
\hline Cipocereus laniflorus & Cereae & E & B & $60-70$ & $30-40$ & $192 \pm 127$ & $17.95 \pm 3.25$ & Q & D, ext. & $\mathrm{Pi}, \mathrm{Ab}, \mathrm{Mu}$ & $\begin{array}{l}\text { Ordones Rego et al. } \\
2012\end{array}$ \\
\hline Echinocereus coccineus & Pachycereae & $\mathrm{Tu}$ & $\mathrm{R}$ & $68.4 \pm 7.6$ & 30 & $94.3+59.4$ & $28.6 \pm 4.6$ & $\mathrm{O}$ & $D, 24 h+$ & $\begin{array}{l}\mathrm{Pi}, \mathrm{Ab}, \text { moscas, } \\
\text { mariposas } \\
\text { diurnas }\end{array}$ & Scobell y Scott 2002 \\
\hline Echinopsis atacamensis & Trichocereeae & $E$ & $B, B-P$ & $124 \pm 2$ & $96 \pm 3$ & $130.154 \pm 15.66$ & - & Q-O & $D, 24 \mathrm{~h}+$ & $\begin{array}{l}\mathrm{Pi}, \mathrm{Ab}, \mathrm{Ma} \\
\text { avispas }\end{array}$ & $\begin{array}{l}\text { de Viana et al. 2001; } \\
\text { Schlumpberger y } \\
\text { Badano } 2005\end{array}$ \\
\hline Marginatocereus marginatus & Pachycereae & $\mathrm{Tu}$ & $\mathrm{R}$ & $34.7 \pm 0.65$ & $15.7 \pm 0.42$ & $87 \pm 18$ & $2548 \pm 0.54$ & $\mathrm{O}$ & $\mathrm{N}$, ext. & $\mathrm{Pi}, \mathrm{Mu}, \mathrm{Ma}$ & Dar et al. 2006 \\
\hline Melocactus andinus & Cereae & $\mathrm{Tu}$ & M & $12.9 \pm 0.4$ & $2.6 \pm 0.1$ & 6.9 & 23.57 & 0 & $D, 24 h+$ & $\begin{array}{l}\mathrm{Pi}, \mathrm{Ab} \\
\text { hormigas, }\end{array}$ & Nassar et al. 2007 \\
\hline
\end{tabular}




\begin{tabular}{|c|c|c|c|c|c|c|c|c|c|c|c|}
\hline & & & & & & & & & & escarabajos & \\
\hline M. curvispinus & Cereae & Tu & M & 25.19 & 2.97 & 163.1 & $29.1-30.2$ & $\mathrm{O}$ & D & $\mathrm{Pi}, \mathrm{Ab}$ & Nassar y Ramírez 2004 \\
\hline M. glaucescens & Cereae & Tu & P-M & $13.5-18$ & $3-3.5$ & $30.4 \pm 3.7$ & $29 \pm 3$ & $\mathrm{O}$ & D & $\mathrm{Pi}, \mathrm{Ab}, \mathrm{Ma}$ & Colaço et al. 2006 \\
\hline M. intortus & Cereae & Tu & P-M & $20-30$ & 18 & $29.7 \pm 9.2$ & $27 \pm 1.8$ & 0 & D & $\mathrm{Pi}, \mathrm{Ab}$, hormigas & $\begin{array}{l}\text { Hunt 2006; Fagua y } \\
\text { Ackerman } 2011\end{array}$ \\
\hline M.paucispinus & Cereae & $\mathrm{Tu}$ & P-M & $12.5-19$ & $2.5-3.5$ & $33.6 \pm 4.1$ & $29 \pm 3$ & $\mathrm{O}$ & D & $\mathrm{Pi}, \mathrm{Ab}, \mathrm{Ma}$ & Colaço et al. 2006 \\
\hline M. schaltzii & Cereae & Tu & M & $17.8 \pm 1.1$ & $2.7 \pm 0.1$ & 4.4 & 23.3 & 0 & D & $\begin{array}{l}\mathrm{Pi}, \mathrm{Ab} \text {, } \\
\text { hormigas, } \\
\text { escarabajos }\end{array}$ & Nassar et al. 2007 \\
\hline M. zehntneri & Cereae & $\mathrm{Tu}$ & $P$ & $15-25$ & $0.3-0.5$ & $42-51$ & $27 \pm 0.57$ & 0 & $\mathrm{D}$ & $\begin{array}{l}\mathrm{Pi} \text {, hormigas, } \\
\text { mariposas } \\
\text { diurnas }\end{array}$ & $\begin{array}{l}\text { Locatelli y Machado } \\
1999\end{array}$ \\
\hline Micranthocereus flaviflorus & Cereae & $\mathrm{Tu}$ & R-P & $12.35 \pm 0.99$ & $1.76 \pm 0.25$ & $5.17 \pm 2.25$ & $23.3 \pm 1.28$ & $\mathrm{O}$ & $D, 24 \mathrm{~h}+$ & $\mathrm{Pi}, \mathrm{Ab}$, otros & $\begin{array}{l}\text { Saleme Aona et al. } \\
2006\end{array}$ \\
\hline M. purpureus & Cereae & $\mathrm{Tu}$ & P-M & $31.88 \pm 1.87$ & $\begin{array}{l}16.35 \pm \\
1.49\end{array}$ & $143.81 \pm 49.32$ & $21.18 \pm 1.54$ & $\mathrm{Q}$ & $\mathrm{N}$, ext. & $\mathrm{Pi}, \mathrm{Mu}, \mathrm{Ab}, \mathrm{Ma}$ & $\begin{array}{l}\text { Saleme Aona et al. } \\
2006\end{array}$ \\
\hline M. streckeri & Cereae & $\mathrm{Tu}$ & $P$ & $13.09 \pm 1.23$ & $1.85 \pm 0.18$ & $7.3 \pm 3.8$ & $19.3 \pm 1.83$ & $\mathrm{O}$ & $\mathrm{N}, 24 \mathrm{~h}+$ & $\begin{array}{l}\mathrm{Pi}, \mathrm{Ab} \\
\text { hormigas, } \\
\text { escarabajos, } \\
\text { mariposas }\end{array}$ & $\begin{array}{l}\text { Saleme Aona et al. } \\
2006\end{array}$ \\
\hline Neobuxbaumia macrocephala & Pachycereae & E & B-P & $50.61 \pm 1.84$ & $16 \pm 3.43$ & - & - & Q & $\mathrm{N}$, ext. & $\mathrm{Pi}, \mathrm{Mu}, \mathrm{Ab}$ & $\begin{array}{l}\text { Valiente-Banuet et al. } \\
\text { 1997; Hunt } 2006\end{array}$ \\
\hline N. mezcalaensis & Pachycereae & E & B-R & $\begin{array}{l}51.58 \pm 4.38 \\
*\end{array}$ & $\begin{array}{l}22.91 \pm \\
3.17^{\star}\end{array}$ & $510 \pm 1770$ & - & $Q$ & $\mathrm{~N}$, ext. & $\mathrm{PI}, \mathrm{Mu}, \mathrm{Ab}$ & $\begin{array}{l}\text { Valiente-Banuet et al. } \\
\text { 1997; Hunt } 2006\end{array}$ \\
\hline Opuntia echios & Opuntieae & $\mathrm{Ta}$ & A & $80-100$ & $50-70$ & $110 \pm 20$ & $22.0 \pm 0.4$ & M & $D, 24 \mathrm{~h}+$ & $\begin{array}{l}\text { Ab, pinzones, } \\
\text { escarabajos }\end{array}$ & $\begin{array}{l}\text { Grant y Grant 1981; } \\
\text { Anderson } 2001\end{array}$ \\
\hline O. helleri & Opuntieae & $\mathrm{Ta}$ & A & $40-80$ & $30-55$ & $110 \pm 20$ & $22.0 \pm 0.4$ & M & $D, 24 \mathrm{~h}+$ & $\begin{array}{l}\text { Ma, pinzones, } \\
\text { ruiseñores, } \\
\text { palomas, } \\
\text { hormigas, grillos }\end{array}$ & $\begin{array}{l}\text { Grant y Grant 1981; } \\
\text { Anderson } 2001\end{array}$ \\
\hline
\end{tabular}




\begin{tabular}{|c|c|c|c|c|c|c|c|c|c|c|c|}
\hline O. palmadora & Opuntieae & $\mathrm{Tu}$ & $\mathrm{R}$ & $30-40$ & $1.2-1.5$ & $20-32$ & $23-27$ & $\mathrm{O}$ & $D, 24 h+$ & $\mathrm{Pi}, \mathrm{Ab}$ & $\begin{array}{l}\text { Locatelli y Machado } \\
1999\end{array}$ \\
\hline O. quimilo & Opuntieae & $\mathrm{Ta}$ & $\mathrm{R}$ & $26^{*}$ & $19.2^{*}$ & - & $35.21 \pm 4.47^{*}$ & $\mathrm{O}$ & $D, 24 \mathrm{~h}+$ & $\mathrm{Pi}, \mathrm{Ab}$ & Díaz y Cocucci 2003 \\
\hline Oreocereus celsianus & Trichocereeae & Tu & $\mathrm{P}$ & $78.1 \pm 10.6$ & $60 \pm 8.6$ & 210 & 26.14 & 0 & $D, 24 h+$ & $\begin{array}{l}\mathrm{Pi}, \mathrm{Ab}, \mathrm{Ma} \\
\text { avispas }\end{array}$ & $\begin{array}{l}\text { Larrea-alcázar y López } \\
2011\end{array}$ \\
\hline Stenocereus queretaroensis & Pachycereae & $E$ & B, B-P & $88.77 \pm 0.61$ & $48 \pm 1.2$ & 560 & $21.37 \pm 3.04$ & C & $\mathrm{N}$, ext. & $\mathrm{Pi}, \mathrm{Mu}, \mathrm{Ab}, \mathrm{Ma}$ & $\begin{array}{l}\text { Ibarra-Cerdaña et al. } \\
\text { 2005; Hunt } 2006\end{array}$ \\
\hline S. thurberi & Pachycereae & $E$ & B & $79.2 \pm 1.5$ & $18.7 \pm 0.8$ & $1980 \pm 180$ & $24-25$ & Q & $\mathrm{N}$, ext. & $\mathrm{Pi}, \mathrm{Mu}, \mathrm{Ab}, \mathrm{Ma}$ & $\begin{array}{l}\text { Fleming et al. 1996; } \\
\text { Bustamante et al. } 2010\end{array}$ \\
\hline $\begin{array}{l}\text { Weberbauerocereus } \\
\text { weberbaueri }\end{array}$ & Trichocereeae & Tu-E & B-R, P-R & $75.7 \pm 1.1$ & $22 \pm 0.12$ & - & - & Q-O & $\mathrm{D}$, ext. & $\mathrm{Pi}, \mathrm{Mu}$ & Sahley 1996 \\
\hline Denmoza rhodacantha & Trichocereeae & $\mathrm{Tu}$ & $M-R$ & $49.1 \pm 7.9$ & $9.9 \pm 2.4$ & - & - & $\mathrm{O}$ & $D, 24 \mathrm{~h}+$ & $\mathrm{Pi}$ & Presente trabajo \\
\hline Echinopsis leucantha & Trichocereeae & $E$ & $B$ & $\begin{array}{l}198.17 \pm \\
13.20\end{array}$ & $\begin{array}{l}113.62 \pm \\
21.74\end{array}$ & $8.02 \pm 9.85$ & - & $Q$ & $\mathrm{~N}$, ext. & $A b$, pinzones & Presente trabajo \\
\hline Cleistocactus baumannii & Trichocereeae & $\mathrm{Tu}$ & $\mathrm{R}$ & $50.20 \pm 9.53$ & $7.19 \pm 1.68$ & $24.93 \pm 20.23$ & $64.5 \pm 4.6$ & $\mathrm{O}$ & D, $24 \mathrm{~h}+$ & $\mathrm{Pi}$ & Presente trabajo \\
\hline C. smaragfiflorus & Trichocereeae & $\mathrm{Tu}$ & R-P & $44.71 \pm 4.09$ & $6.97 \pm 1.89$ & $26.48 \pm 21.34$ & $62.2 \pm 3.79$ & 0 & $D, 24 \mathrm{~h}+$ & $\mathrm{Pi}, \mathrm{Ab}$ & Presente trabajo \\
\hline
\end{tabular}




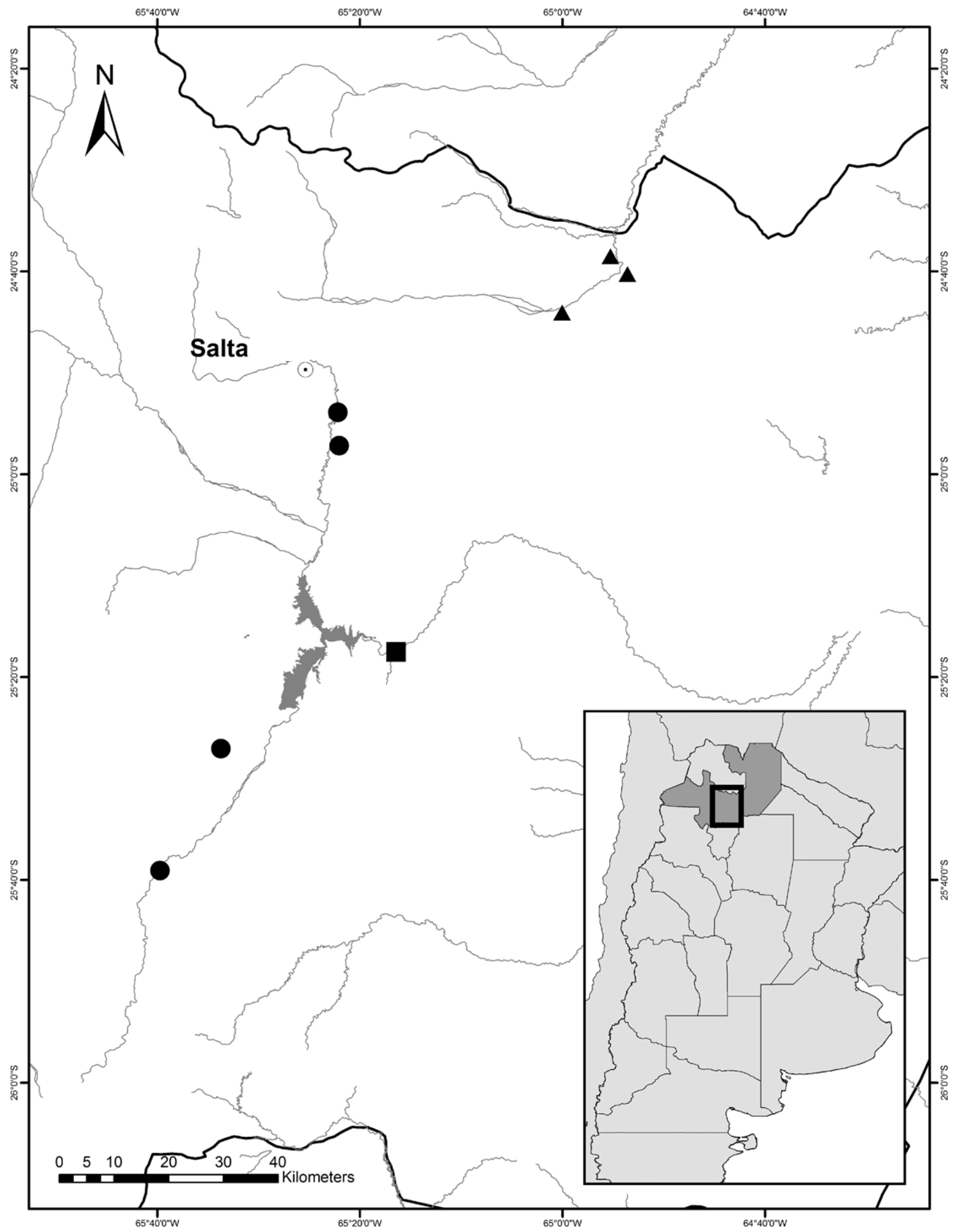

Figura 1. Área de estudio incluyendo el sitio La Bodeguita (cuadrado) y otras poblaciones de Cleistocactus baumannii (triángulos) y C. smaragdiflorus (círculos) en Salta, Argentina. 

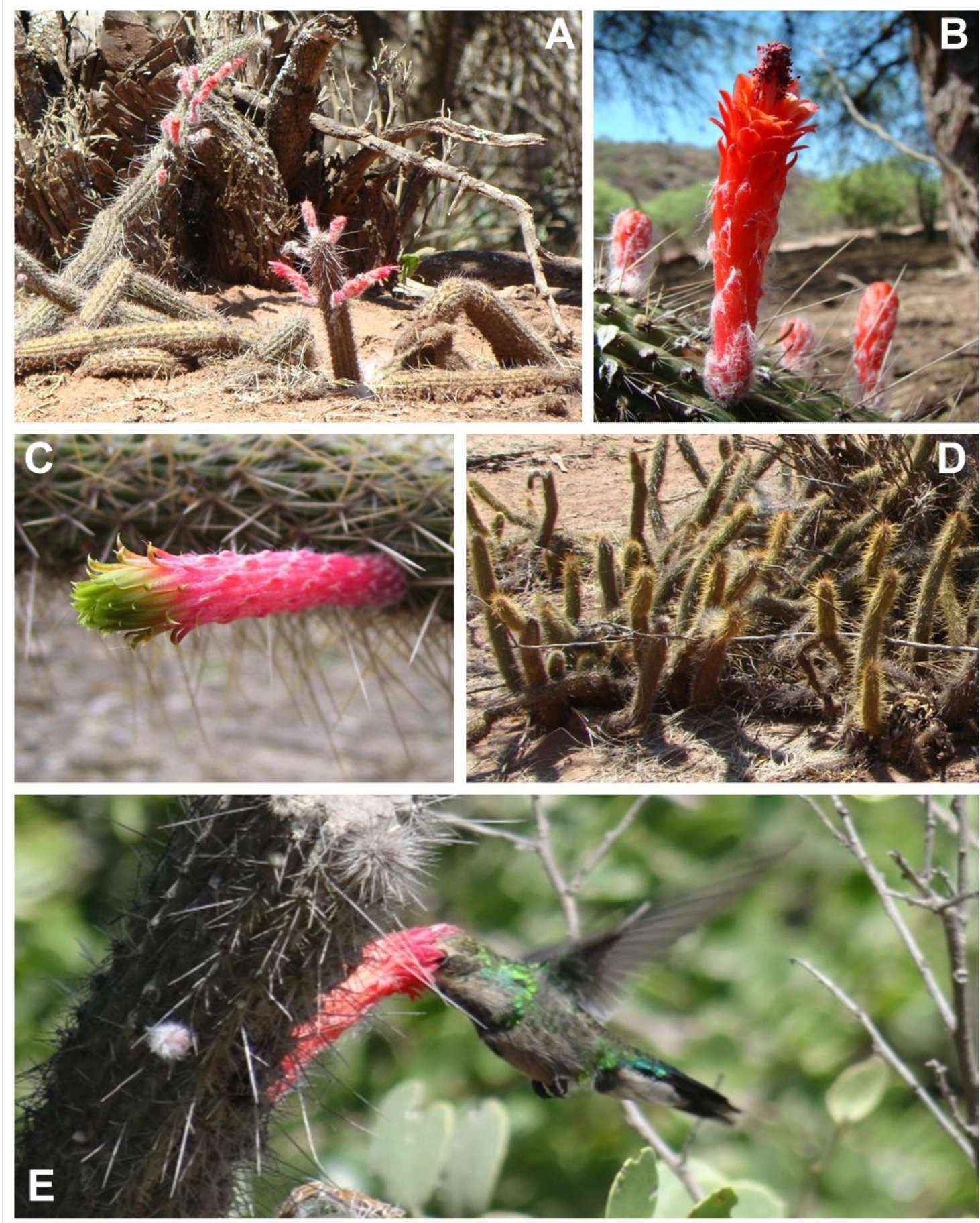

Figura 2. Individuos de Cleistocactus baumannii (A) con flores rojas tubulares (B). Flores rosadas tubulares con tépalos apicales verdes de Cleistocactus smaragdiflorus (C) y un grupo de individuos (D). El picaflor Chlorostilbon lucidus introduciendo su cabeza en una flor de Cleistocactus baumannii (E). Fotos: P. Gorostiague. 


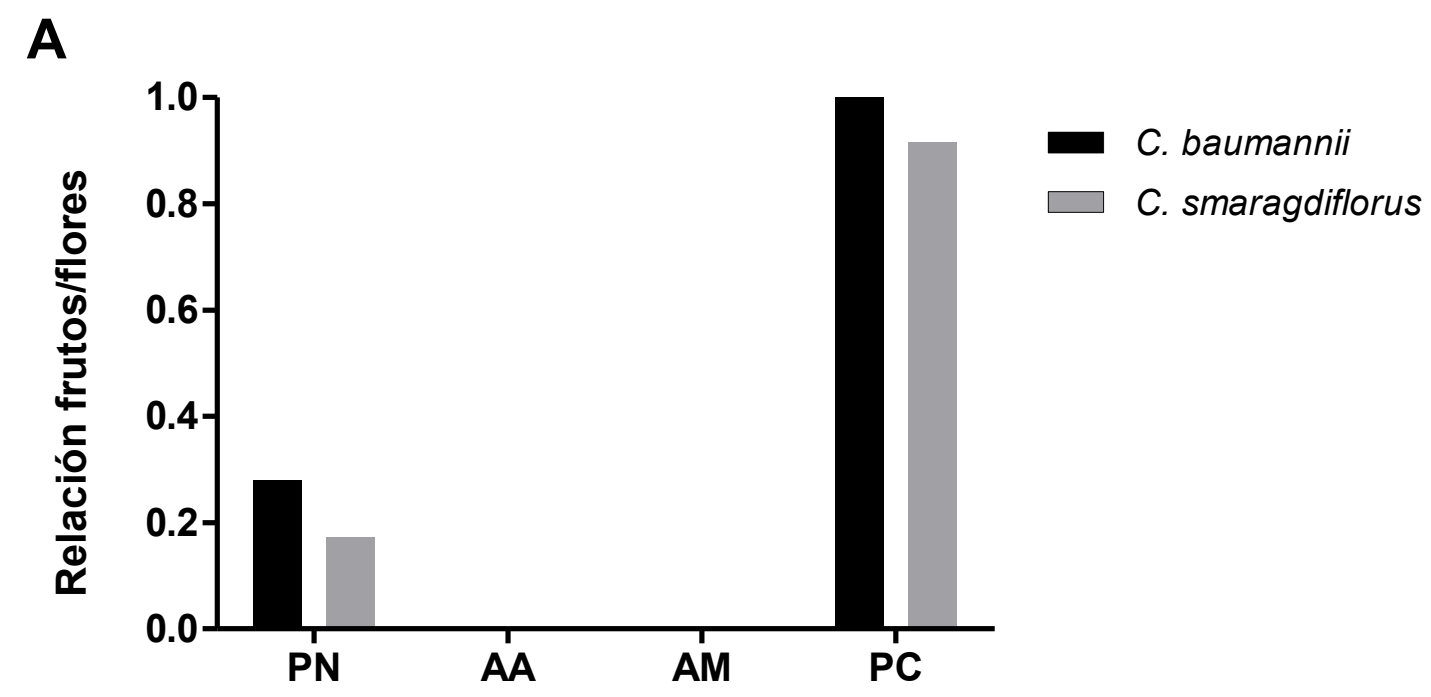

B

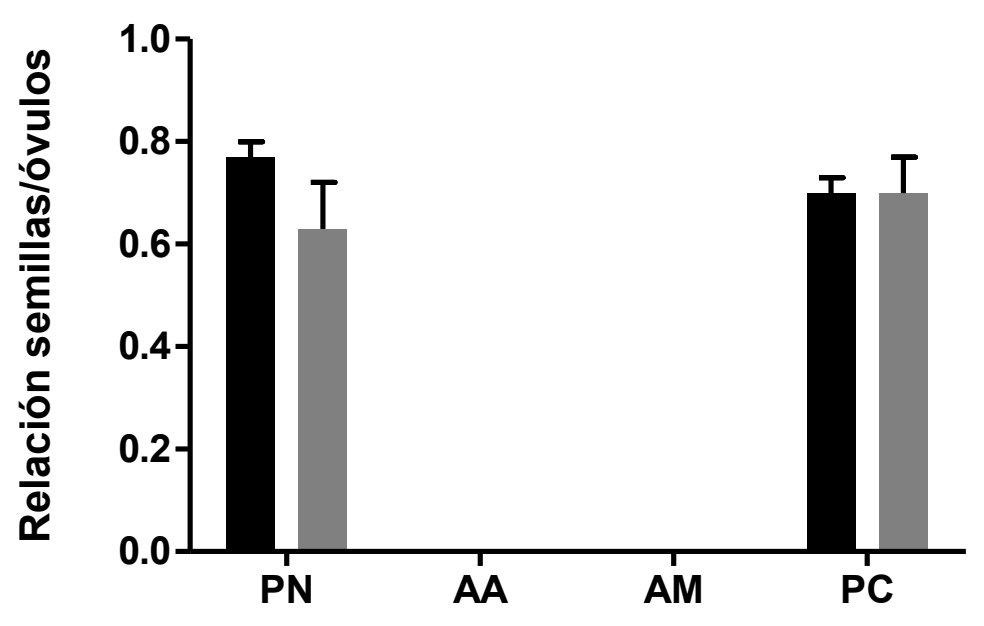

Figura 3. Relación frutos/flores (A) y relación semillas/óvulos (B) obtenida en cada uno de los tratamientos del experimento por el que se determinó el sistema reproductivo de Cleistocactus baumannii y C. smaragdiflorus. PN: polinización natural, AA: auto-polinización automática, AM: auto-polinización manual, PC: polinización cruzada manual. 


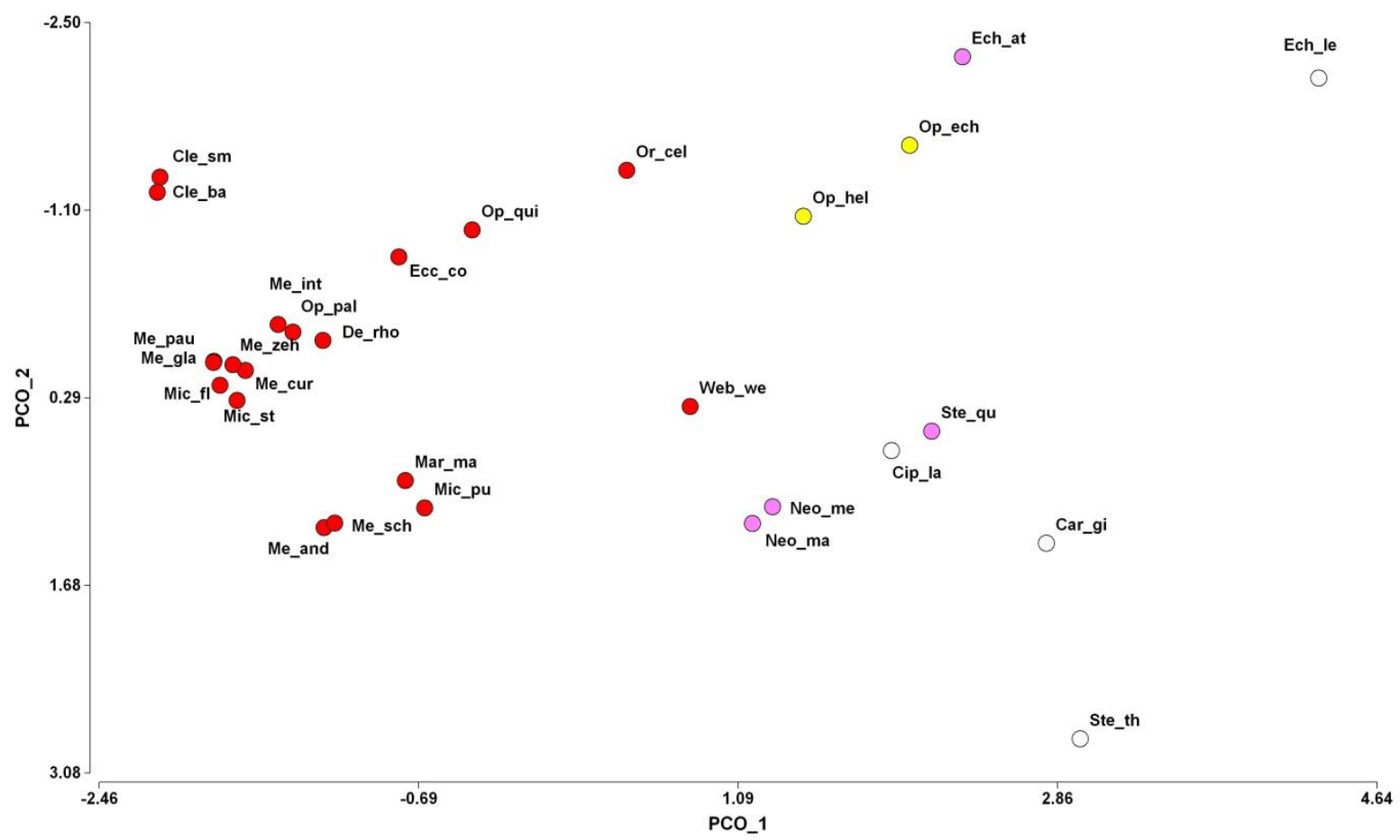

Figura 4. Análisis de coordenadas principales multi-escalar para los rasgos florales asociados a la polinización incluyendo a todas las especies estudiadas de cactáceas en los que se registró polinización por aves. Los rasgos florales utilizados fueron: largo promedio de la flor, ancho promedio de la flor, producción promedio de néctar por flor, concentración promedio de azúcares, tiempo de antesis, forma floral y color de la corola. 


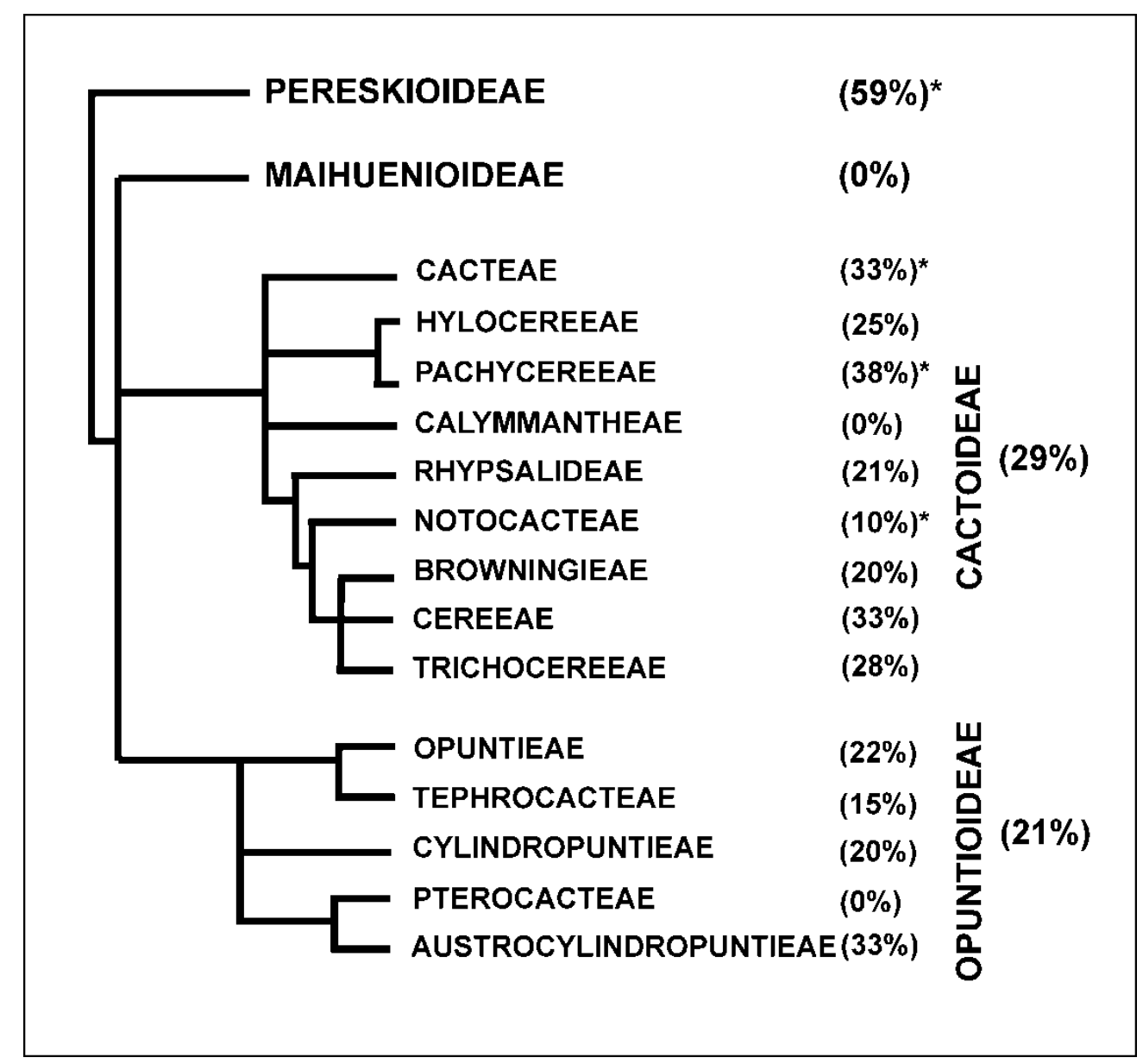

Figura 5. Proporción de especies con flores rojizas en cada subfamilia y tribu de la familia Cactaceae. Los grupos fueron organizados en base en la filogenia propuesta por Bárcenas et a. (2011). * indica que la proporción de especies con flores rojizas fue significativamente diferente a lo esperado por azar $(p<0.005)$. 


\section{CAPÍTULO III}

Efecto del cambio climático sobre la distribución geográfica de 12 especies de cactáceas del Noroeste de Argentina y el acople espacial con sus polinizadores 


\section{INTRODUCCIÓN}

En la actualidad, la biodiversidad que existe en la Tierra enfrenta serias amenazas que son producto de cambios ambientales como consecuencia de la actividad del hombre (Primack 2008, Barnosky et al. 2011). Se puede clasificar a estas amenazas en cinco grandes grupos que incluyen la contaminación, las invasiones biológicas, la sobreexplotación, el cambio en el uso del suelo y el cambio climático (Sodhi 2010). Si bien el cambio climático constituye una amenaza potencial que tendría consecuencias más severas en el futuro, numerosos estudios han demostrado que este fenómeno ya está alterando la distribución y abundancia de los organismos (Parmesan y Yohe 2003, Parmesan 2006).

Los cambios del clima a lo largo del tiempo constituyen una variabilidad natural que ha ocurrido repetidas veces durante la historia de la Tierra. Sin embargo, en la actualidad se considera que estos cambios, asociados principalmente al calentamiento global ocasionado por el efecto invernadero, constituyen una amenaza para el hombre y para toda la biodiversidad (Sodhi 2010, IPCC 2014). El efecto invernadero, que consiste en la acumulación de calor en la atmósfera por parte de algunos gases como el dióxido de carbono, metano, óxido nitroso y clorofluorocarbonos, es también un proceso natural que existe desde hace millones de años; sin embargo, se estima que la concentración de estos gases en la atmósfera aumentó considerablemente desde los inicios de la revolución industrial, acelerando el calentamiento global. Por lo tanto, la principal causa del cambio climático actual es de origen antropogénico, como consecuencia de las emisiones de gases (principalmente, dióxido de carbono) a la atmósfera (Lovejoy 2010, IPCC 2014). Algunas de las evidencias de este fenómeno son el aumento en el nivel del mar, el derretimiento de las grandes masas de hielo y el aumento promedio global de la temperatura del aire y de los océanos. Éstas evidencias indican que la velocidad con la que se produce el cambio climático en la actualidad es muy superior a la registrada a lo largo de la historia de la vida en la Tierra, lo que tendría consecuencias severas sobre la salud humana, la seguridad alimentaria, los servicios ecosistémicos y la biodiversidad en general, aumentando considerablemente el riesgo de extinción de un gran número de especies (Barnosky et al. 2011, IPCC 2014). 
La evidencia actual sugiere que, para muchas especies, los efectos del cambio climático serían negativos, ya que se predicen reducciones en el tamaño de las poblaciones, pudiendo llegar a la extinción de muchas de ellas, e inclusive se ha sugerido que pueden llegar a ocasionar la extinción global de algunas especies (Bellard et al. 2012). Por lo tanto, resulta relevante generar información acerca de los efectos potenciales que puede ocasionar el cambio climático sobre la biodiversidad, con el fin de establecer estrategias de conservación tanto in situ como ex situ.

En términos generales, se ha indicado que el cambio climático tiene la capacidad de generar disrupciones en las interacciones entre especies, debido a un desacople temporal (asociado a la fenología, Memmott et al. 2007, Parmesan 2007) o espacial (asociado al desplazamiento, expansión o contracción de sus rangos geográficos, Schweiger et al. 2010) entre las especies que interactúan. Esta disrupción sería especialmente importante para las interacciones mutualistas en las que las especies dependen en algún grado de la interacción con otras especies para cumplir con alguna de las fases de su ciclo de vida, tal como el caso de la polinización en plantas auto-incompatibles, las que dependen de los polinizadores para la producción de frutos y semillas. En estas últimas especies, las estimaciones de los efectos del cambio climático deberían evaluar si las especies tendrán o no un desacople con respecto a las especies con las cuales interactúan.

Una herramienta ampliamente utilizada para estimar los efectos del cambio climático sobre la distribución de las especies es la modelación de la distribución de especies basada en el nicho ecológico. Para construir estos modelos se necesitan dos tipos de insumos: capas de datos ambientales y datos de presencia (o en algunos casos también ausencias) de las especies bajo estudio (Elith et al. 2010). Una vez obtenida la información acerca de las variables ambientales que determinan el nicho de una especie, es posible proyectarla sobre nuevas capas de datos distintas a las originales. Por lo tanto, estos modelos son de gran utilidad para predecir la distribución de una especie en el presente, en el pasado y en futuro, bajo diferentes escenarios de cambio climático (Elith et al. 2010). Los modelos de distribución son de suma utilidad para predecir los potenciales 
cambios en la distribución geográfica de las especies como consecuencia del cambio climático, permitiendo inclusive estimar cuantitativamente la magnitud de estos cambios.

Tradicionalmente, se ha considerado que los factores abióticos determinan la distribución de las especies a escala geográfica global. Sin embargo, se ha sugerido que las interacciones bióticas también tienen la capacidad de definir los límites geográficos de algunas especies no sólo a escala local, sino también a escala regional o global (Wisz et al. 2013). La distribución de una especie no sólo está determinada por variables ambientales como las del clima, sino que también existen interacciones bióticas (por ejemplo, la competencia y los mutualismos; Soberón 2007), que deben tenerse en cuenta como parte del nicho ecológico de la especie. A pesar de esto, el papel de las interacciones bióticas en los estudios de cambio climático permanece poco estudiado (Araújo y Luoto 2007, HilleRisLambers et al. 2013). Debido a la importancia del ambiente biótico, la estimación de los efectos del cambio climático sobre la distribución de una especie utilizando los modelos de distribución debería tener en cuenta no sólo las variables climáticas sino también a la presencia de las especies con las que establecen interacciones mutualistas (Araújo y Luoto 2007).

Las cactáceas son un grupo de plantas que se distribuyen a lo largo del continente americano, y típicamente habitan ambientes áridos y semiáridos. La familia constituye un grupo prioritario para acciones de conservación debido a que una gran proporción de sus especies se encuentra amenazada por diversos factores, entre los cuales se encuentran el cambio en el uso del suelo y la sobreexplotación por colecta ilegal (Ortega-Baes et al. 2010, Goettsch et al. 2015). Al igual que para toda la biodiversidad, el cambio climático antropogénico constituye una amenaza potencial para esta familia de plantas; sin embargo, no existen evaluaciones de su efecto al presente. La diversificación evolutiva de esta familia se ha asociado al proceso de aridización del continente americano originado como consecuencia del levantamiento orográfico, como por ejemplo el de la cordillera de los Andes en América del Sur (Hershkovitz y Zimmer 1997, Hernández-Hernández et al. 2014). Bajo un escenario de calentamiento global en el que muchos ambientes podrían volverse más áridos (IPCC 2014), el cambio climático no 
tendría efectos negativos sobre la distribución de especies de cactáceas, al menos para aquellas que habitan las regiones más áridas del hemisferio oeste. Por lo tanto, uno de los posibles efectos de estos cambios sería la expansión del rango geográfico de las especies de esta familia, ocupando nuevas áreas que antes no estaban disponibles. Sin embargo, la posibilidad de que este grupo de plantas colonice nuevos ambientes en escenarios de cambio climático futuro, dependerá además, de la posibilidad de que las especies mutualistas con las cuales interactúa positivamente también puedan expandir sus rangos geográficos de distribución.

Las especies de cactáceas se caracterizan por establecer relaciones mutualistas con otros organismos, asociados a los procesos de polinización, dispersión de semillas y establecimiento de nuevos individuos (Pimienta-Barrios y del Castillo 2002, Godínez-Álvarez et al. 2003). Particularmente, la polinización constituye un proceso fundamental para la reproducción de una gran proporción de las especies de la familia debido a dependen exclusivamente de sus polinizadores para la producción de frutos y semillas (Capítulo I). Por lo tanto, la presencia o ausencia de los animales que polinizan las flores en las nuevas áreas de distribución, podría condicionar la presencia o ausencia de una especie de cactácea. Se ha sugerido que el cambio climático puede tener efectos diferenciales sobre la distribución geográfica de las especies que participan de una interacción mutualista como la polinización, generando desacople espacial en las relaciones planta-polinizador (Drezner et al. 2014, Giannini et al 2013, HilleRisLambers et al. 2013).

Bajo este marco teórico, el objetivo del presente capítulo fue analizar el impacto del cambio climático sobre la distribución geográfica de las cactáceas de la Tribu Trichocereeae del noroeste de Argentina, evaluando el acople (o desacople) espacial con sus polinizadores. Se pusieron a prueba las siguientes hipótesis: 1) debido a que el aumento en las condiciones de aridez en el continente americano favoreció la radiación de las cactáceas en el pasado, el efecto del cambio climático se expresará en un incremento del rango geográfico de las especies de este grupo de plantas; y 2) el cambio climático generará un desacople espacial entre las cactáceas y sus polinizadores. Para poner a prueba 
estas hipótesis se utilizaron como modelo de estudio 12 especies de cactáceas de la tribu Trichocereeae del noroeste de Argentina, que contemplan todos los tipos de sistema de polinización conocidos en la actualidad para las especies de esta tribu. Específicamente, se respondieron las siguientes preguntas: a) ¿qué nivel de acople espacial presenta en la actualidad la distribución geográfica de 12 especies de cactáceas de la tribu Trichocereeae que habitan el noroeste de Argentina con la de sus polinizadores?; b) ¿cuál será la distribución geográfica de estas especies de cactáceas bajo escenarios de cambio climático futuro, para los años 2050 y 2070?; y c) ¿cuál será la magnitud del desacople espacial originado por el cambio climático futuro entre las especies de cactáceas y sus polinizadores? Hasta donde se conoce, este es el primer estudio que evalúa el impacto del cambio climático sobre la distribución geográfica de un grupo de especies de la familia Cactaceae en América del Sur.

\section{MATERIALES Y MÉTODOS}

\section{Especies bajo estudio}

El grupo seleccionado para este estudio fue el de las especies de cactáceas de la tribu Trichocereeae que habitan en el noroeste de Argentina. Fueron incluidas en este análisis todas las especies de la tribu para las cuales se conoce el sistema reproductivo y la identidad de sus polinizadores (Capítulo I), sumando un total de 12 especies. Las mismas fueron: Cleistocactus baumannii, C. smaragdiflorus, Denmoza rhodacantha, Echinopsis albispinosa, E. ancistrophora, E. atacamensis, E. haematantha, E. leucantha, E. schickendantzii, E. terscheckii, E. thelegona y Gymnocalycium saglionis. Además, se incluyeron un total de 19 especies de animales polinizadores y 11 géneros de animales que se comportan como polinizadores de cada una de estas especies de plantas, incluyendo aves, abejas, avispas y mariposas nocturnas (Capítulo I).

\section{Datos de presencia de las especies}

El primer insumo de información para construir los modelos de distribución de especies son los datos de presencia de cada una de las especies. Para esto, se construyó una base de datos para cada especie que incluyó las coordenadas geográficas de cada registro de presencia. Para las especies de cactáceas, los registros de presencia fueron obtenidos a partir de datos de herbario y de datos 
tomados a campo durante viajes del equipo de trabajo de la institución (OrtegaBaes, datos no publicados). Esta base de datos contiene un total de 384 puntos de registro para las 12 especies incluidas. Para cada especie, se disponía de un mínimo de 12 y un máximo de 93 puntos de registro de presencia (Fig. 1).

Los animales que se comportan como polinizadores de las cactáceas bajo estudio incluyeron especies de aves, abejas, avispas y mariposas nocturnas (Capítulo I). Los registros de presencia de estas especies se obtuvieron de una base de datos mundial de acceso libre en el portal GBIF (Global Biodiversity Information Facility, GBIF 2016). Con el objetivo de evitar la incorporación de errores en los datos de presencia, las bases de datos descargadas del portal fueron revisadas y filtradas teniendo en cuenta la sinonimia taxonómica, la fuente de cada registro, y los posibles errores en las coordenadas geográficas, corroborando los registros con un software de SIG (Qgis 2011). Para los casos en los que no se contaba con información de la especie de polinizador, se modeló la distribución del género correspondiente. En los casos en los que tampoco se contaba con datos de presencia del género, se descartó la especie del análisis.

\section{Datos climáticos}

Las variables ambientales utilizadas para construir los modelos se obtuvieron del portal de acceso libre WorldClim (Hijmans et al. 2005). Se utilizaron 19 variables denominadas bioclimáticas, que consisten en combinaciones de las precipitaciones y la temperatura, y una variable topográfica de altitud. Las variables bioclimáticas utilizadas fueron: temperatura media anual, rango medio diurno (media por mes, máxima temperatura - mínima temperatura), isotermalidad, estacionalidad de la temperatura (desvío estándar *100), temperatura máxima del mes más cálido, temperatura mínima del mes más frío, rango anual de la temperatura, temperatura media del trimestre más húmedo, temperatura media del trimestre más seco, temperatura media del trimestre más cálido, temperatura media del trimestre más frío, precipitación anual, precipitación del mes más húmedo, precipitación del mes más seco, estacionalidad de las precipitaciones (coeficiente de variación), precipitación del trimestre más húmedo, precipitación del trimestre más seco, precipitación del trimestre más cálido, y precipitación del trimestre más frío. 
EI IPCC (Panel Intergubernamental de Cambio Climático) plantea en su informe del año 2014 una serie de posibles escenarios de cambio climático para el siglo XXI, dependiendo de las medidas que se implementen a lo largo del siglo para disminuir las emisiones de gases de efecto invernadero y del desarrollo económico de los países. Para el presente trabajo se seleccionaros dos potenciales escenarios de cambio climático: RCP4.5 (escenario intermedio con una baja emisión de gases, y con implementación de mitigaciones para evitar el cambio climático), y RCP8.5 (escenario con una alta emisión de gases de efecto invernadero; IPCC 2014). Se seleccionaron dos escenarios diferentes debido a que, tal como espeficfica el IPCC, no hay escenarios futuros que tengan una mayor probabilidad de ocurrir. De esta manera, se intentó contemplar esta incertidumbre al menos en cierta medida. Se construyeron mapas de distribución para el año 2050 (promedio del período 2041 - 2060) y para el año 2070 (promedio del período 2061 - 2080). Se utilizaron las proyecciones construidas a partir del modelo de circulación global ACCESS1, desarrollado por el instituto australiano CSIRO debido a que éstas han sido utilizadas para numerosos estudios de modelado del cambio climático, sobre todo asociados al hemisferio sur. Las proyecciones del clima futuro bajo escenarios de cambio climático fueron obtenidas del portal WolrdClim (Hijmans et al. 2005).

\section{Modelación de la distribución potencial de las especies}

Loa mapas de distribución potencial de cada especie en el presente y el futuro se construyeron utilizando el software MaxEnt, un programa desarrollado por Phillips et al. (2006) para estimar la distribución probabilística de un objeto, tal como la distribución espacial de una especie. El método utilizado para la validación de los modelos fue el de cross-validación, construyendo diez réplicas para cada especie y utilizando el mapa promedio para el análisis. Para probar el desempeño del programa, se utilizó el valor AUC (área bajo la curva) de cada modelo, en la cual se representa la especificidad y la sensibilidad en cada punto del área utilizada. Se consideró que si el valor del AUC fue superior a 0.7 , el modelo es efectivo para predecir la distribución de la especie (Phillips et al. 2006).

El umbral de corte a partir del cual se consideró que la especie se encuentra presente fue el de "máxima especificidad más sensibilidad" para los 
puntos de entrenamiento; es decir, un umbral basado en el poder de predicción de ausencias y presencias a partir de los datos de presencia de la especie utilizados inicialmente por el software. El área sobre la que se construyeron los modelos para las cactáceas fue diferente para cada especie, y seleccionó teniendo en cuenta las ecorregiones en las que se registró la presencia de la especie (siguiendo las ecorregiones propuestas por Olson y Dinerstein, 2002) más un área buffer de 100 kilómetros alrededor de los límites de las mismas. De esta manera, se restringió el modelado al área que puede ocupar la especie para evitar errores de sobre-predicción y valores de AUC artificiales (Lobo et al. 2007). No fue posible utilizar el mismo criterio para los modelos de distribución de las especies de polinizadores, debido al menor grado de conocimiento de los ambientes que habitan, y a las bases de datos de registro incompletas. Para estas especies, el área utilizada para modelar la distribución correspondió al territorio de América del Sur continental, dentro de la cual la mayoría de las especies (o géneros) contaban con registros de presencia.

\section{Escenarios de dispersión}

La capacidad de una especie para dispersarse y ocupar nuevos ambientes que antes no estaban disponibles a su rango geográfico es un factor que debe tenerse en cuenta al momento de construir los modelos futuros y predecir los cambios en la distribución de especies. Esto puede variar según el tipo de organismo del que se trate, por ejemplo, los animales pueden moverse con distintos grados de vagilidad, mientras que las plantas, al ser organismos sésiles, no pueden moverse y dependen de la distancia a la que puedan dispersar sus propágulos y de la velocidad a la que éstos se desarrollen y comiencen a producir nuevos propágulos. Para este trabajo, se tuvieron en cuenta dos escenarios de dispersión para todas las especies: uno de dispersión ilimitada, en el que las especies tienen la capacidad de ocupar todos los nuevos ambientes en los que tengan una alta favorabilidad; y otro escenario de dispersión cero, en el que las especies sólo ocuparán los ambientes que actualmente habitan, sin posibilidad de conquistar nuevos ambientes.

\section{Estimación del impacto del cambio climático sobre la distribución de las especies}


Para evaluar el impacto del cambio climático sobre el rango de distribución de las 12 especies de cactáceas se tuvieron en cuenta dos variables: la diferencia proporcional del área ocupada en los escenarios futuros con respecto al área en el presente, y la dirección y magnitud del cambio de posición del centroide (centro de una figura geométrica irregular como lo es el área de distribución de una especie) del rango geográfico, medido en kilómetros de distancia. De esta forma, se puede estimar cuál será el área ganada o perdida por cada especie en los escenarios futuros, qué tanto se desplazará el rango, y en qué dirección. Adicionalmente, se calculó cual sería la diferencia en el área ocupada en el presente y en los escenarios futuros teniendo en cuenta sólo la porción del rango geográfico en la que están presentes los polinizadores. Para esto, se construyó, para cada especie de cactácea, un mapa que representa la presencia de todos los polinizadores para los cuales se construyó la distribución geográfica. A partir de esto, se superpuso el mapa de presencia de cada especie de cactácea y se obtuvo el área en la que estaría presente tanto la cactácea como alguno de sus polinizadores; es decir, el acople espacial en el que se daría la interacción mutualista. Esto se realizó para el presente y para los escenarios futuros.

\section{RESULTADOS}

El software MaxEnt tuvo un buen desempeño en predecir la distribución potencial de todas las especies, con valores de AUC (área bajo la curva) mayores a 0.8 en casi todos los casos para las especies de cactáceas, tanto para los puntos de entrenamiento como para los puntos de prueba obtenidos mediante la validación cruzada (Tabla 1). Con respecto a las especies de polinizadores, MaxEnt también tuvo un buen desempeño de acuerdo a los valores de AUC de cada especie (Tabla 2), con valores mayores a 0.9 para la mayoría de los modelos. Para algunas especies, se obtuvieron valores cercanos a 0.6 , los cuales corresponden a especies (o géneros) para los cuales se contaba con un bajo número de registros de presencia. Sin embargo, estos valores indican que el modelo sigue teniendo un mejor poder predictivo que una distribución al azar en el área de estudio.

La distribución potencial actual y futura bajo diferentes escenarios de cambio climático de cada especie de cactácea estudiada se presenta en las 
Figuras 2-13. En las figuras $14-25$ se encuentra representada la proporción del rango geográfico ganado o perdido por cada especie de cactácea bajo diferentes escenarios de cambio climático futuro con respecto al rango geográfico actual. En promedio, se predijo que las especies de cactáceas incluidas en el análisis perderían un $21.57 \pm 29.93 \%$ (media $\pm D E$ ) de su rango geográfico en el año 2050 y un $22.66 \pm 55.49 \%$ en el año 2070 . La especie para la cual se predijo la mayor pérdida de rango geográfico en promedio, fue Echinopsis albispinosa $(80 \%$ de pérdida del rango de distribución), seguida de Cleistocactus baumanni (68.17\%) y E. thelegona (63.75\%). Contrariamente, las especies con menor pérdida de rango geográfico fueron E. terscheckii (3.77\%) y E. schickendantzii (5.81\%). Tres especies se verían favorecidas con una expansión de su rango bajo escenarios futuros de cambio climático: Denmoza rhodacantha (51.47\%), E. leucantha (8.13\%) y E. atacamensis (6\%).

Teniendo en cuenta la porción del rango geográfico compartida con alguno de sus polinizadores, se predijo una pérdida del $18.38 \pm 29.61 \%$ del rango en el año 2050 y un $28.18 \pm 34.69 \%$ en 2070 (Figs. 14-25). Las especies para las cuales se predijo un aumento en el tamaño del rango geográfico fueron $E$. atacamensis (20.29\%), E. leucantha (10.09\%) y Gymnocalycium saglionis (7.19\%). Para el resto de las especies, se predijo una reducción del tamaño del rango geográfico. Las especies afectadas en mayor medida fueron $E$. albispinosa (67.19\%), C. baumannii (60.51\%) y E. thelegona (57.99\%), mientras que las especies afectadas en menor medida fueron $E$. terscheckii (2.64\%) y $E$. schickendantzii (0.37\%).

Al considerar un escenario sin dispersión, en el que las especies sólo mantienen el área que ocupan en la actualidad, se predijo una pérdida de rango geográfico para todas las especies. En promedio, las especies perdieron un 33.43 $\pm 23.29 \%$ de su rango en 2050 y un $42.94 \pm 27.03 \%$ en 2070 . Teniendo en cuenta el área que comparten con sus polinizadores, las especies perdieron un $27.98 \pm$ $24.52 \%$ de su rango en 2050 y un $38.88 \pm 29.05 \%$ en 2070 (Figs. 14-25). Las especies que se vieron afectadas en mayor medida con una reducción de su rango geográfico bajo este escenario fueron $E$. albspinosa, $C$. baumannii y $E$. thelegona, tanto cuando se consideró la distribución de las cactáceas (80.05, 
78.92 y $64.51 \%$ respectivamente) como cuando se consideró la distribución limitada por la presencia de sus polinizadores (67.34, 73.95 y $58.80 \%$ respectivamente).

Para todas las especies analizadas se predijo un desplazamiento del centroide de su distribución geográfica bajo escenarios de cambio climático futuro (Tabla 3). Para la mayoría de las especies (75\%), el desplazamiento del rango en promedio fue hacia el sur. El promedio total de desplazamiento del centroide del rango geográfico para todas las especies de cactáceas estudiadas fue de $77.32 \pm$ $80.47 \mathrm{Km}$ (media $\pm \mathrm{DE}$ ), siendo de $60.5 \pm 57.41 \mathrm{Km}$ para el año 2050 y $94.10 \pm$ $96.70 \mathrm{Km}$ para el año 2070. Entre las especies para las cuales se predijo un mayor desplazamiento de su rango geográfico en promedio se encuentran $D$. rhodacantha $(217.7 \mathrm{Km})$, E. leucantha $(114.45 \mathrm{Km})$ y G. sagiolins $(113.43 \mathrm{Km})$; mientras que las especies con menor desplazamiento fueron E. terscheckii (22.28 $\mathrm{Km})$ y C. smaragdiflorus (33.47 Km).

La diferencia entre el rango geográfico de cada especie de cactácea y el rango que esta especie comparte con alguno de sus polinizadores (Figs. 14-25), fue considerado como una medida del acople espacial entre estas especies. Se consideró que hubo un alto nivel de acople espacial si éste fue mayor al $80 \%$. En seis especies (C. smaragdiflorus, $E$. atacamensis, E. haematantha, $E$. schickendantzii, E. terschecki y E. thelegona), se encontró un alto nivel de acople espacial para el presente. Bajo escenarios de cambio climático futuro, se predijo que todas estas especies aumentarán su acople espacial con los polinizadores, a excepción de C. smaragdiflorus. Sin embargo, en promedio, esta especie continuaría presentando un alto nivel de acople. El resto de las especies ( $C$. baumannii, D. rhodacantha, E. albispinosa, E. ancistrophora, E. leucantha y G. saglionis) presentaron un nivel de acople espacial con sus polinizadores inferior al $80 \%$ en el presente (Figs. 14-25). Bajo escenarios de cambio climático futuro, tres de estas especies (C. baumannii, E. albispinosa y G. saglionis.) aumentaron su nivel de acople espacial, superando el $80 \%$ en promedio. Mientras que las otras tres especies mantuvieron ( $E$. ancistrophora y $E$. leucantha) o disminuyeron ( $D$. rhodacantha) el nivel de acople espacial con sus polinizadores.

\section{DISCUSIÓN}


El presente estudio constituye el primero en estimar el rango geográfico de un grupo de especies de la familia Cactaceae a partir de modelos de distribución que incluyeron variables abióticas (climáticas), y teniendo en cuenta adicionalmente a las interacciones bióticas. Si bien numerosos estudios plantean la importancia de incorporar el ambiente biótico a la predicción del nicho ecológico de las especies (Araujo y Louto 2007, Heikkinen 2007), este enfoque se ha implementado en muy pocos estudios (Hillrieslamber 2013, Schweiger et al. 2010). Particularmente, las cactáceas establecen interacciones mutualistas que afectan directamente a su dinámica poblacional. Entre las más importantes se pueden citar a la polinización, la dispersión de semillas y la facilitación para el establecimiento de semillas (Pimienta-Barrios y del Castillo 2002, Godínez-Álvarez et al. 2003). En este trabajo, se incorporó un factor del ambiente biótico a la estimación del nicho ecológico: la interacción planta-polinizador. Esta interacción es vital para muchas especies de la familia, y especialmente para el grupo de especies incluidas en este análisis, ya que todas las especies son auto-incompatibles; es decir, que necesitan de los animales polinizadores para la producción de frutos y semillas (Capítulo I). La información acerca del grado de dependencia que tienen las especies de los animales que polinizan sus flores, así como su identidad, resulta fundamental para ser considerada en la estimación del nicho ecológico de las especies.

Con el objetivo de incorporar a la polinización como interacción que limita la distribución de este grupo de plantas, se predijo el rango geográfico de cada especie de cactácea a partir de variables ambientales, y luego se evaluó en qué porción de ese rango geográfico se encontraba alguna de las especies que se desempeña como polinizador de la misma. Se asumió que la porción del rango geográfico en la que no está presente alguno de los polinizadores no representaría hábitat viable para la especie de cactácea. Esto quiere decir que habría sitios en los que las condiciones abióticas permitirían el desarrollo de la especie, pero no contarían con las interacciones mutualistas necesarias, representadas en este caso por la presencia de sus polinizadores (Soberón 2007, Schweiger et al. 2012). De acuerdo a los resultados de distribución potencial para el presente, hubo seis especies de cactáceas que presentaron un acople espacial alto con sus 
polinizadores (más del $80 \%$ de su rango geográfico compartido con presencia de los mismos). Para las otras seis especies de cactáceas, la proporción del rango compartido con sus mutualistas fue menor, y en algunas especies llegó hasta el $50 \%$ de su rango geográfico total. Esto indica que la distribución actual de las cactáceas estaría limitada, en mayor o menor medida, por la presencia de los polinizadores de los que depende. Si bien los factores abióticos (por ejemplo, las variables climáticas) determinarían la distribución de una especie en un nivel jerárquico mayor (Holland y Molina-Freaner 2013), estos resultados resaltan la importancia de incorporar las interacciones biológicas para ajustar esta distribución a las condiciones ecológicas reales de las que depende la presencia de las mismas (Soberón 2007).

Teóricamente, los sitios en los que no se da la interacción con los polinizadores pero sí las condiciones climáticas del nicho de la especie, no serían hábitats viables para la cactácea, porque no cuenta con agentes vectores de polen que permitan su reproducción. Sin embargo, se debe tener en cuenta que en la práctica, los polinizadores de cada especie incluida en este análisis podrían variar en el tiempo y en el espacio. Por ejemplo, en los sitios para los que se predice ausencia de polinizadores, podrían existir otros animales que polinizan las flores y que no han sido registrados aún. El grupo bajo estudio ha demostrado presentar sistemas de polinización generalistas para un gran número de especies (Capítulos I y II), y esto es, por lo tanto, factible de ocurrir. Adicionalmente, si bien los mapas de distribución obtenidos representan el área donde está presente la especie, hay que destacar que los mismos fueron construidos a partir de un mapa probabilístico de favorabilidad. El umbral de corte a partir del cual se considera que la especie está presente es una estimación y podría dejar afuera algunos sitios con baja probabilidad de ocurrencia en los que la especie de polinizador en realidad sí está presente. Por lo tanto, a partir de los resultados obtenidos en este trabajo, surgen una serie de interrogantes que deberían ser abordados en estudios futuros. Primero, se deben evaluar los sitios en los que se predijo la presencia de la cactácea pero no de sus polinizadores, para corroborar la presencia de la misma; en el caso de que la cactácea esté presente, se deben identificar los animales que polinizan sus flores en estos sitios; y finalmente, evaluar si los modelos de 
distribución para los polinizadores son lo suficientemente sólidos para predecir su rango geográfico. Esto pone de manifiesto la necesidad de realizar validación de campo para los modelos construidos. Estas validaciones han sido muy importantes para detectar poblaciones no conocidas para especies con prioridades de conservación, como por ejemplo, especies raras y endémicas (Rebelo y Jones 2010, Rinnhofer et al. 2012) que es el caso de las cactáceas. Además, serían útiles para evaluar la viabilidad de un área geográfica en función de la presenciaausencia de una especie mutualista con la que la especie blanco interactúa y no sólo por la disponibilidad de hábitat en una dimensión abiótica.

Frente a un cambio en las condiciones climáticas en el ambiente que habita, una población de una determinada especie tiene tres posibles destinos: adaptarse a las nuevas condiciones (a partir de, por ejemplo, un proceso de selección natural en un tiempo muy corto), extinguirse localmente, o migrar hacia nuevos ambientes cuyas condiciones permitan su subsistencia (Parmesan 2006). Sin embargo, se ha sugerido que la adaptación local en un tiempo corto sería algo difícil de alcanzar, y la mayoría de los estudios asumen que no es viable (Parmesan 2006, Corlett y Westcott 2013). Por lo tanto, los cambios en el rango geográfico debido al cambio climático deben ser contemplados en la toma de decisiones asociadas a medidas de conservación, y resulta importante estimar la magnitud de estos cambios para el grupo de organismos que se desea conservar.

Para todas las especies de cactáceas evaluadas, se predijeron cambios en las características del rango geográfico en el futuro bajo escenarios de cambio climático con respecto a la distribución actual. Sin embargo, no todas las especies se comportaron de la misma manera. Para la mayoría de las especies de cactáceas se predijo una reducción del tamaño del rango geográfico, en mayor o menor medida (hasta el $80 \%$ del rango actual para algunas especies). Sin embargo, para tres especies (Denmoza rhodacantha, Echinopsis leucantha y $E$. atacamensis) se predijo una expansión del rango geográfico bajo escenarios futuros de cambio climático. Por lo tanto, el cambio climático tendría efectos diferenciales sobre las especies, siendo positivos para algunas y negativos para otras. Los resultados sugieren que las especies que habitan ambientes semiáridos se verían afectadas negativamente en comparación con las especies que habitan 
ambientan áridos, lo que sustenta la idea original de que la aridización es un proceso que permitirá la expansión de al menos algunas especies de esta familia, tal como ha ocurrido en el proceso histórico de radiación del grupo (Hershkovitz y Zimmer 1997, Hernández-Hernández et al. 2014). Futuros estudios, con un mayor número de especies deberían confirmar los resultados obtenidos aquí para el grupo de cactáceas estudiadas.

Es importante resaltar que la expansión del rango predicho para algunas especies se daría bajo un escenario de dispersión ilimitada; es decir, donde las especies tienen la capacidad de ocupar todos los nuevos ambientes disponibles. La velocidad con la que las especies pueden llegar a ocupar estos ambientes es un factor difícil de estimar, y algunos trabajos sugieren que muchas especies de plantas no podrían alcanzar la velocidad del cambio climático futuro (Corlett y Westcott 2013). Esto es especialmente importante para el grupo bajo estudio, dada las limitaciones registradas para el establecimiento de nuevos individuos en muchas especies de la familia (Godínez-Álvarez et al. 2003). El establecimiento vía semilla es un proceso raro en cactáceas, ya que de la gran cantidad de semillas que se producen anualmente unas pocas germinan, y de las pocas plántulas emergidas, la mayoría no sobreviven al primer año de vida (GodínezAlvarez et al. 2003). Adicionalmente, es importante tener en cuenta los sistemas de dispersión de semillas de las especies bajo estudio. En general, se conoce poco sobre este proceso en la familia y, particularmente para las especies que habitan nuestro país, no existen estudios sobre este tópico. Observaciones de campo sugieren que existen diferencias entre las especies en relación a los animales que pueden actuar como vectores de semillas. Así, las semillas de especies de cactáceas columnares como Echinopsis atacamensis y E. terscheckii serían dispersadas por animales con mayor vagilidad como las aves, en contraposición a las semillas de especies globosas como $E$. albispinosa, $E$. ancistrophora y $E$. haematantha cuyas semillas serían dispersadas por animales con una vagilidad reducida como hormigas (Ortega-Baes y Gorostiague, datos no publicados). En este contexto, es importante considerar los escenarios de dispersión cero, en el que las especies sólo mantendrían la porción de su rango geográfico que ocupan actualmente. Bajo este escenario, todas las especies se 
verían afectadas negativamente con una reducción del tamaño de su rango geográfico. Para las cactáceas incluidas en este análisis, se estima una pérdida de gran parte de su rango geográfico, que en promedio oscila entre un 27 - $42 \%$.

Finalmente, se observó que los efectos (tanto positivos como negativos) sobre el rango geográfico de las especies estudiadas fueron de mayor magnitud para el escenario más severo de cambio climático, en comparación con el escenario intermedio que prevé una reducción en las emisiones de gases de efecto invernadero a través de la implementación de políticas para mitigar el cambio climático (IPCC 2014). Esto evidencia que los efectos del cambio climático antropogénico sobre la biodiversidad dependerán en gran medida de las medidas que se tomen a lo largo de las próximas décadas (Schweiger et al. 2012)

Los resultados indicaron que, en promedio, no habría un desacople espacial entre el rango geográfico de las especies de cactáceas y el de sus polinizadores como consecuencia del cambio climático. Contrariamente a lo esperado, y para muchas especies, la proporción de rango compartido fue mayor bajo escenarios de cambio climático futuro. Las especies que presentaron un alto nivel de acople espacial en el presente, lo mantuvieron en escenarios futuros; mientras que las especies que tenían un bajo nivel de acople espacial (menor al $80 \%$ ), presentaron valores mayores de acople bajo escenarios futuros. Sólo en una especie, la endémica Denmoza rhodacantha, se obtuvo un resultado opuesto; es decir, se predijo una disminución en el rango geográfico compartido con sus polinizadores en escenarios climáticos futuros. Particularmente, esta especie constituye el único caso de especialización ecológica (sensu Ollerton et al. 2007), siendo polinizada exclusivamente por el picaflor Oreotrochilus leucopleurus. Esta especialización en la polinización podría provocar que la especie se vea amenaza en mayor medida por el desacople espacial que provocaría el cambio climático (Schweiger et al. 2012). En otros estudios, se ha propuesto que los mutualismos más especializados corren un mayor riesgo de desacople entre las especies interactuantes, siendo por lo tanto más vulnerables a los efectos negativos del cambio climático (Schweiger et al. 2012, Rafferty et al. 2015). En el presente trabajo se apoya esta idea ya que el único caso de especialización en el sistema 
de polinización conocido para estas especies en Argentina, se predijo un alto nivel de desacople espacial en escenarios climáticos futuros.

El presente trabajo pone en evidencia la importancia de incorporar las interacciones bióticas a las proyecciones que evalúan los efectos del cambio climático sobre la distribución espacial de las especies, las que serán importantes para establecer estrategias de conservación (ver Capítulo IV). Para esto, es necesario contar con información de base sobre las interacciones que establecen las especies, así como sobre el grado de dependencia de las mismas. Además, son necesarios estudios que evalúen el papel de otras interacciones que también son fundamentales para el desarrollo del ciclo de vida de las plantas más allá de la polinización, tales como la dispersión de semillas por medio de aves o insectos, o la facilitación para el establecimiento de semillas por medio de plantas nodriza. Finalmente, la disrupción en las interacciones debido al cambio climático fue evaluada aquí en base al desacople espacial entre las especies que interactúan, y se debería avanzar a estudios que contemplen el desacople temporal o fenológico que este fenómeno puede generar sobre los mutualismos. Para esto, es necesario obtener información a campo a partir de estudios a largo plazo.

\section{REFERENCIAS BIBLIOGRÁFICAS}

Araújo, M.B., Luoto, M. (2007). The importance of biotic interactions for modelling species distributions under climate change. Global Ecology and Biogeography 16: 743-753.

Barnosky A.D., Matzke N., Tomiya S., Wogan G.O., Swartz B., Quental T.B., Marshall C., McGuire J.L., Lindsey E.L., Maguire K.C., Mersey B., Ferrer E.A. (2011). Has the Earth's sixth mass extinction already arrived? Nature 471: 51-57.

Bellard C., Bertelsmeier C., Leadley P., Thuiller W., Courchamp F. (2012) Impacts of climate change on the future of biodiversity. Ecology letters 15: 365-377.

Corlett R.T., Westcott D.A. (2013) Will plant movements keep up with climate change? Trends in ecology \& evolution 28: 482-488.

Drezner T.D. (2014) The keystone saguaro (Carnegiea gigantea, Cactaceae): a review of its ecology, associations, reproduction, limits, and demographics. Plant ecology 215(6): 581-595. 
Elith J., Kearney M., Phillips S. (2010). The art of modelling range-shifting species. Methods in ecology and evolution 1(4): 330-342.

Giannini T.C., Acosta A.L., da Silva C.I., de Oliveira P.E.A.M., Imperatriz-Fonseca V.L., Saraiva A.M. (2013) Identifying the areas to preserve passion fruit pollination service in Brazilian Tropical Savannas under climate change. Agriculture, ecosystems \& environment 171: 39-46.

Godínez-Alvarez H.O., Valverde T., Ortega-Baes P. (2003) Demographic trends in the Cactaceae. Botanical Review 69: 173-203.

Goettsch B., Hilton-Taylor C., Cruz-Piñón G., Duffy J.P., Frances A., Hernández H.M., et al. (2015). High proportion of cactus species threatened with extinction. Nature plants 1: 15142.

Heikkinen R.K., Luoto M., Virkkala R., Pearson R.G. Körber J.H. (2007) Biotic interactions improve prediction of boreal bird distributions at macro-scales. Global Ecology and Biogeography 16: 754-763.

Hernández-Hernández T., Brown J.W., Schlumpberger B.O., Eguiarte L.E., Magallón S. (2014). Beyond aridification: multiple explanations for the elevated diversification of cacti in the New World Succulent Biome. New phytologist 202: 1382-1397.

Hershkovitz M.A., Zimmer E.A. (1997) On the evolutionary origins of the cacti. Taxon 1997: 217-232.

Hijmans R.J., Cameron S.E., Parra J.L., Jones P.G., Jarvis A. (2005) Very high resolution interpolated climate surfaces for global land areas. International Journal of Climatology 25: 1965-1978.

HilleRisLambers J., Harsch M.A., Ettinger A.K., Ford K.R., Theobald E.J. (2013). How will biotic interactions influence climate change-induced range shifts? Annals of the New York Academy of Sciences 1297: 112-125.

Holland J.N., Molina-Freaner F. (2013) Hierarchical effects of rainfall, nurse plants, granivory and seed banks on cactus recruitment. Journal of vegetation science 24: 1053-1061.

IPCC (2014) Cambio climático 2014: Informe de síntesis. Contribución de los Grupos de trabajo I, II y III al Quinto Informe de Evaluación del Grupo Intergubernamental de Expertos sobre el Cambio Climático [Equipo 
principal de redacción, R.K. Pachauri y L.A. Meyer (eds.)]. IPCC, Ginebra, Suiza, $157 \mathrm{p}$.

Lobo J.M., Jiménez-Valverde A., Real R. (2008) AUC: a misleading measure of the performance of predictive distribution models. Global ecology and Biogeography 17: 145-151.

Lovejoy T.E. (2010) Climate Change. En: Sodhi, N.S.(ed.) Conservation biology for all. Oxford University Press.

Memmott J., Craze P.G., Waser N.M., Price M.V. (2007) Global warming and the disruption of plant-pollinator interactions. Ecology Letters 10: 710-717.

Ollerton J., Killick A., Lamborn E., Watts S., Whiston M. (2007) Multiple meanings and modes: on the many ways to be a generalist flower. Taxon 53: 717-728.

Olson D.M., E. Dirnestein. (2002) The global 200: Priority ecoregions for global conservation. Annals Missouri Botanical Garden 89: 129-224.

Ortega-Baes P., Shüring S., Sajama J., Sotola E., Alonso Pedano M., Bravo S., Godínez-Alvarez H. (2010) Diversity and conservation in the cactus family. En: Ramawat K. (ed). Desert Plants. Biology and Biotechnology. Springer.

Parmesan C., Yohe G. (2003) A globally coherent fingerprint of climate change impacts across natural systems. Nature 421(6918): 37-42.

Parmesan C. (2006). Ecological and evolutionary responses to recent climate change. Annual Review of Ecology, Evolution, and Systematics 37: 637669.

Parmesan, C. (2007). Influences of species, latitudes and methodologies on estimates of phonological response to global warming. Global Change Biology 13: 1860-1872.

Phillips S.J., Anderson R.P., Schapire R.E. (2006) Maximum entropy modeling of species geographic distributions. Ecological Modelling 190: 231-259.

Pimienta-Barrios E., del Castillo R.F. (2002) Reproductive biology. En: Nobel P.S. (ed) Cacti: biology and uses. University of California Press. pp. 163-183.

Primack R.B. (2008) A Primer of Conservation Biology. Sinauer, Sunderland, USA.

QGIS Development Team. (2011) QGIS Geographic Information System. Open Source Geospatial Foundation. URL http://qgis.osgeo.org

Rafferty N.E., CaraDonna P.J., Bronstein J.L. (2015) Phenological shifts and the 
fate of mutualisms. Oikos 124: 14-21.

Rebelo H., Jones G. (2010) Ground validation of presence-only modelling with rare species: a case study on barbastelles Barbastella barbastellus (Chiroptera: Vespertilionidae). Journal of Applied Ecology 47: 410-420.

Rinnhofer L.J., Roura-Pascual N., Arthofer W., Dejaco T., Thaler-Knoflach B., Wachter G.A., Schlick-Steiner, B.C. (2012) Iterative species distribution modelling and ground validation in endemism research: an Alpine jumping bristletail example. Biodiversity and Conservation 21: 2845-2863.

Schweiger O., Biesmeijer J.C., Bommarco R., Hickler T., Hulme P.E., Klotz S., Kühn I., Moora M., Nielsen A., Ohlemüller R., Petanidou T., Potts S.G., Pysek P., Stout J.C., Sykes M.T., Tscheulin T., Vilà M., Walther G., Westphal C., Winter M., Zobel M., Settele J. (2010) Multiple stressors on biotic interactions: how climate change and alien species interact to affect pollination. Biological Reviews 85: 777-795.

Schweiger O., Heikkinen R.K., Harpke A., Hickler T., Klotz S., Kudrna O., Kühn I., Pöyry J., Settele J. (2012). Increasing range mismatching of interacting species under global change is related to their ecological characteristics. Global Ecology and Biogeography 21: 88-99.

Soberón J. (2007) Grinnellian and Eltonian niches and geographic distributions of species. Ecology letters 10: 1115-1123.

Sodhi, N.S. (2010). Conservation biology for all. Oxford University Press.

Wisz M.S., Pottier J., Kissling W.D., Pellissier L., Lenoir J., Damgaard C.F., et al. (2013) The role of biotic interactions in shaping distributions and realised assemblages of species: implications for species distribution modelling. Biological Reviews 88(1): 15-30. 
Tabla 1. Valores del área bajo la curva (AUC) obtenidos para cada especie de cactácea a partir del modelo construido por MaxEnt, utilizando los puntos de entrenamiento (con los que se construye el modelo) y los puntos de prueba (el 10\% de los puntos apartados para la validación cruzada).

\begin{tabular}{lrr}
\hline Especie & AUC de Entrenamiento & AUC de Prueba \\
\hline Cleistocactus baumannii & 0.86 & 0.67 \\
C. smaragdiflorus & 0.94 & 0.92 \\
Denmoza rhodacantha & 0.98 & 0.94 \\
Echinopsis albispinosa & 0.99 & 0.99 \\
E. ancistrophora & 0.98 & 0.96 \\
E. atacamensis & 0.97 & 0.95 \\
E. haematantha & 0.98 & 0.95 \\
E. leucantha & 0.95 & 0.90 \\
E. schickendantzii & 0.89 & 0.84 \\
E. terscheckii & 0.97 & 0.95 \\
E. thelegona & 0.99 & 0.99 \\
E. thionantha & 0.99 & 0.99 \\
Gymnocalycium saglionis & 0.97 & 0.92 \\
\hline
\end{tabular}


Tabla 2. Valores del área bajo la curva (AUC) obtenidos para cada especie o género de polinizadores a partir del modelo construido por MaxEnt, utilizando los puntos de entrenamiento (con los que se construye el modelo) y los puntos de prueba (el $10 \%$ de los puntos apartados para la validación cruzada).

\begin{tabular}{lrr}
\hline Especie & AUC de Entrenamiento & AUC de Prueba \\
\hline Amazilia chionogaster & 0.97 & 0.96 \\
Apis mellifera & 0.91 & 0.79 \\
Arhysosage ochracea & 0.99 & 0.98 \\
Augochloropsis & 0.91 & 0.83 \\
Bombus atratus & 0.97 & 0.95 \\
B. morio & 0.97 & 0.93 \\
Brachyglossula communis & 0.97 & 0.95 \\
Chlorostilbon lucidus & 0.92 & 0.89 \\
Citheronia & 0.91 & 0.87 \\
Colibri coruscans & 0.92 & 0.91 \\
Condica & 0.83 & 0.67 \\
Diadasia & 0.92 & 0.88 \\
Erinnyis ello & 0.88 & 0.84 \\
Manduca & 0.91 & 0.84 \\
Manduca sexta & 0.95 & 0.88 \\
Megachile & 0.90 & 0.83 \\
Ophisma tropicalis & 0.88 & 0.70 \\
Oreotrochilus leucopleurus & 0.99 & 0.98 \\
Patagona gigas & 0.93 & 0.93 \\
Phrygilus gayi & 0.95 & 0.95 \\
Plebeia & 0.89 & 0.82 \\
Polybia & 0.90 & 0.80 \\
Ptilothrix tricolor & 0.98 & 0.95 \\
Sappho sparganura & 0.98 & 0.97 \\
Trichothurgus & 0.98 & 0.92 \\
Xylocopa ordinaria & 0.98 & 0.97 \\
X. splendidula & 0.98 & 0.95 \\
Xylocopa & 0.89 & 0.84 \\
Xylophanes tersa & 0.90 & 0.78 \\
Zale & 0.77 & 0.60 \\
\hline & &
\end{tabular}


Tabla 3. Desplazamiento del centroide del rango geográfico (distancia medida en kilómetros y dirección con respecto a los puntos cardinales) bajo diferentes escenarios de cambio climático futuro de 12 especies de cactáceas. NE: Noreste; NO: Noroeste; SE: Sudeste; SO: Sudoeste.

\begin{tabular}{|c|c|c|c|c|c|c|c|c|c|c|c|c|c|c|c|}
\hline \multirow[b]{3}{*}{ Especie } & \multicolumn{6}{|c|}{ Año 2050} & \multicolumn{6}{|c|}{ Año 2070} & \multirow{2}{*}{\multicolumn{3}{|c|}{ Promedio }} \\
\hline & \multicolumn{3}{|c|}{ RCP 4,5 } & \multicolumn{3}{|c|}{ RCP 8,5 } & \multicolumn{3}{|c|}{ RCP 4,5} & \multicolumn{3}{|c|}{ RCP 8,5 } & & & \\
\hline & $\begin{array}{l}\text { Magnitud } \\
(\mathrm{Km})\end{array}$ & Ángulo & $\begin{array}{l}\text { Direcci } \\
\text { ón }\end{array}$ & $\begin{array}{l}\text { Magnitud } \\
(\mathrm{Km})\end{array}$ & Ángulo & $\begin{array}{l}\text { Direcci } \\
\text { ón }\end{array}$ & $\begin{array}{l}\text { Magnitud } \\
(\mathrm{Km})\end{array}$ & Ángulo & $\begin{array}{l}\text { Direcci } \\
\text { ón }\end{array}$ & $\begin{array}{l}\text { Magnitu } \\
\mathrm{d}(\mathrm{Km})\end{array}$ & Ángulo & $\begin{array}{l}\text { Direcci } \\
\text { ón }\end{array}$ & $\begin{array}{l}\text { Magnitud } \\
(\mathrm{Km})\end{array}$ & Ángulo & $\begin{array}{l}\text { Direcci } \\
\text { ón }\end{array}$ \\
\hline $\begin{array}{l}\text { Cleistocactus } \\
\text { baumannii }\end{array}$ & 96.11 & -165.56 & so & 103.40 & -158.93 & so & 79.36 & -163.52 & so & 110.99 & -159.94 & so & 97.47 & -161.99 & so \\
\hline $\begin{array}{l}\text { C. smaragdiflorus } \\
\text { Denmoza }\end{array}$ & 32.50 & -104.51 & so & 44.73 & -92.11 & so & 21.04 & -110.38 & SO & 35.61 & -117.83 & so & 33.47 & -106.21 & SO \\
\hline $\begin{array}{l}\text { rhodacantha } \\
\text { Echinopsis }\end{array}$ & 233.87 & -82.93 & SE & 26.03 & -82.13 & SE & 243.00 & -86.16 & SE & 367.88 & -86.73 & SE & 217.70 & -84.49 & SE \\
\hline albispinosa & 43.47 & -135.48 & so & 43.29 & -136.99 & so & 39.51 & -149.18 & SO & 72.13 & -138.44 & so & 49.60 & -140.02 & So \\
\hline E. ancistrophora & 17.23 & 166.43 & NO & 75.16 & -96.94 & so & 42.13 & -117.73 & SO & 86.52 & -103.48 & so & 55.26 & -37.93 & SE \\
\hline E. atacamensis & 132.94 & 94.40 & NO & 12.28 & -104.54 & so & 25.28 & 86.14 & NE & 34.01 & -69.09 & SE & 51.13 & 1.73 & $\mathrm{NE}$ \\
\hline E. haematantha & 64.99 & 86.49 & $\mathrm{NE}$ & 29.05 & -97.11 & so & 41.47 & -81.07 & SE & 109.56 & -89.63 & SE & 61.27 & -45.33 & SE \\
\hline E. leucantha & 38.34 & 86.55 & $\mathrm{NE}$ & 203.36 & -80.14 & SE & 64.27 & 64.62 & NE & 151.85 & -78.78 & SE & 114.45 & -1.94 & SE \\
\hline E. schickendantzii & 29.89 & 99.71 & NO & 53.75 & 86.41 & $\mathrm{NE}$ & 43.34 & 87.75 & NE & 79.64 & 97.28 & NO & 51.65 & 92.79 & NO \\
\hline E. terscheckii & 9.40 & 53.16 & $\mathrm{NE}$ & 22.32 & -108.54 & so & 25.29 & 82.60 & NE & 32.13 & 106.92 & NO & 22.28 & 33.54 & $\mathrm{NE}$ \\
\hline $\begin{array}{l}\text { E. thelegona } \\
\text { Gymnocalycium }\end{array}$ & 36.57 & -133.52 & so & 53.04 & -142.20 & so & 64.82 & -141.79 & SO & 86.21 & -145.11 & so & 60.16 & -140.66 & SO \\
\hline saglionis & 16.43 & -150.64 & so & 34.94 & 157.94 & NO & 40.53 & -131.56 & SO & 361.83 & -101.36 & so & 113.43 & -56.41 & SE \\
\hline
\end{tabular}




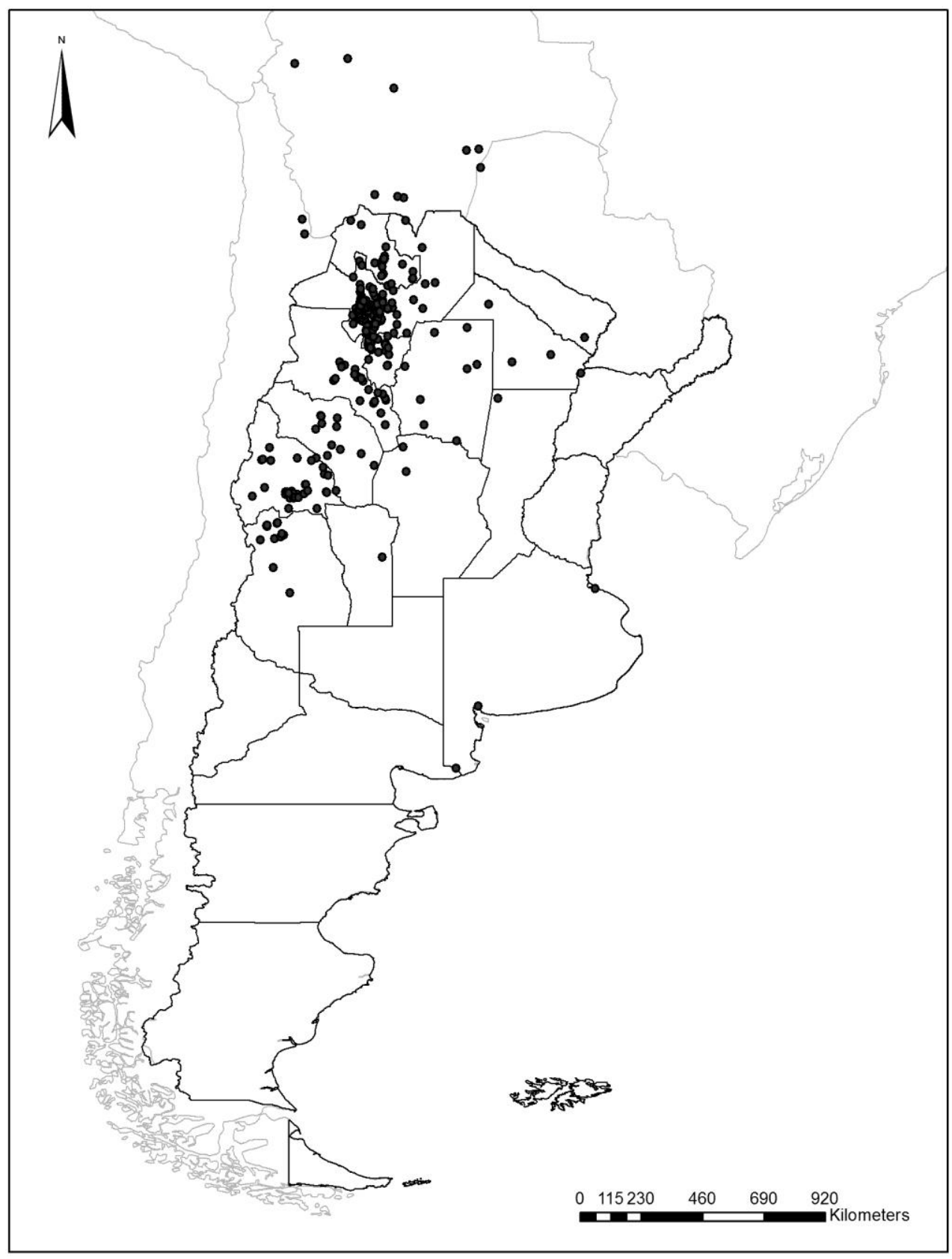

Figura 1. Puntos de registro para las 12 especies de cactáceas incluidas en el análisis. 

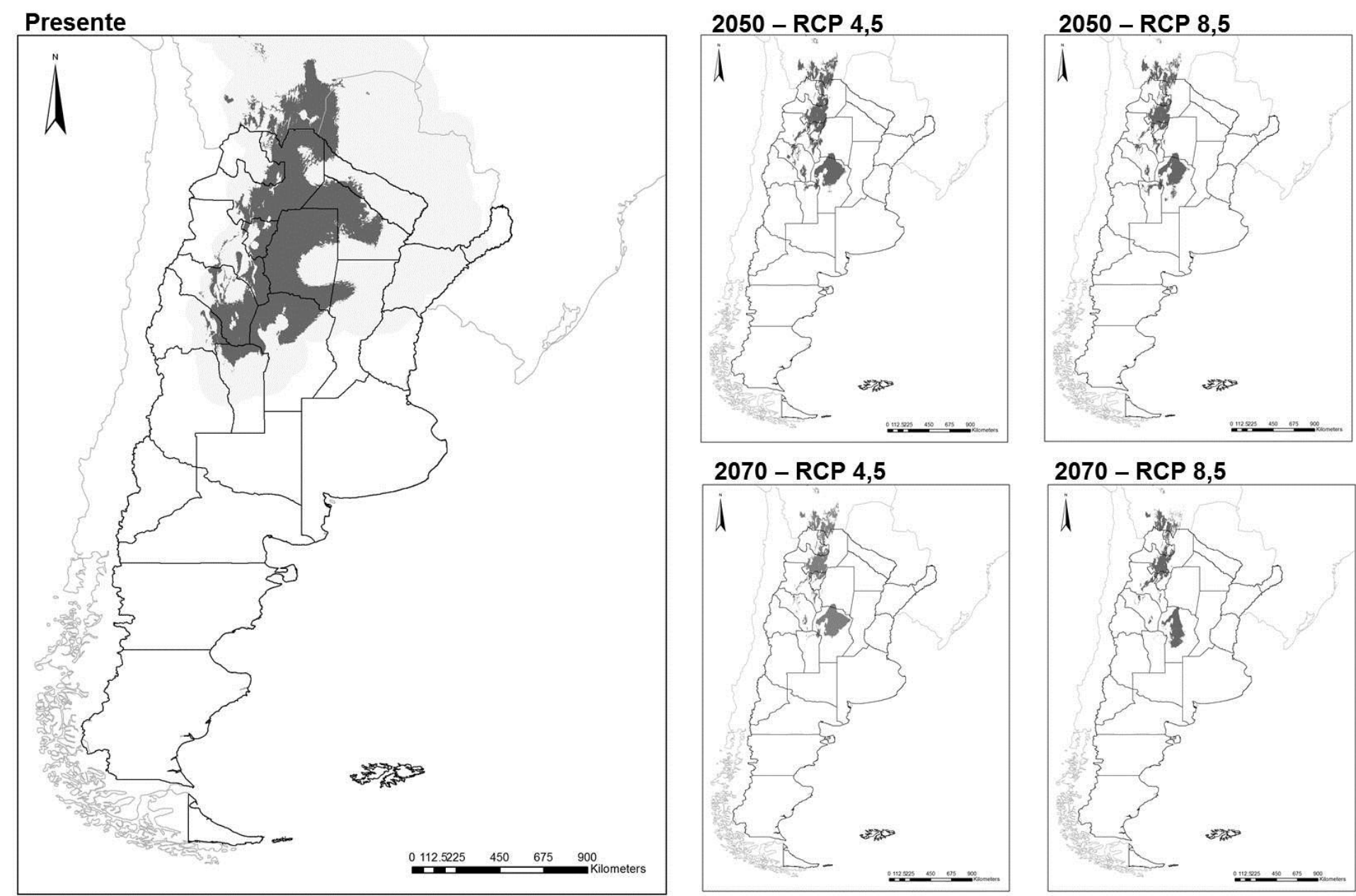

Figura 2. Distribución geográfica potencial en el presente y en el futuro (años 2050 y 2070) bajo diferentes escenarios de cambio climático de la cactácea Cleistocactus baumannii. 

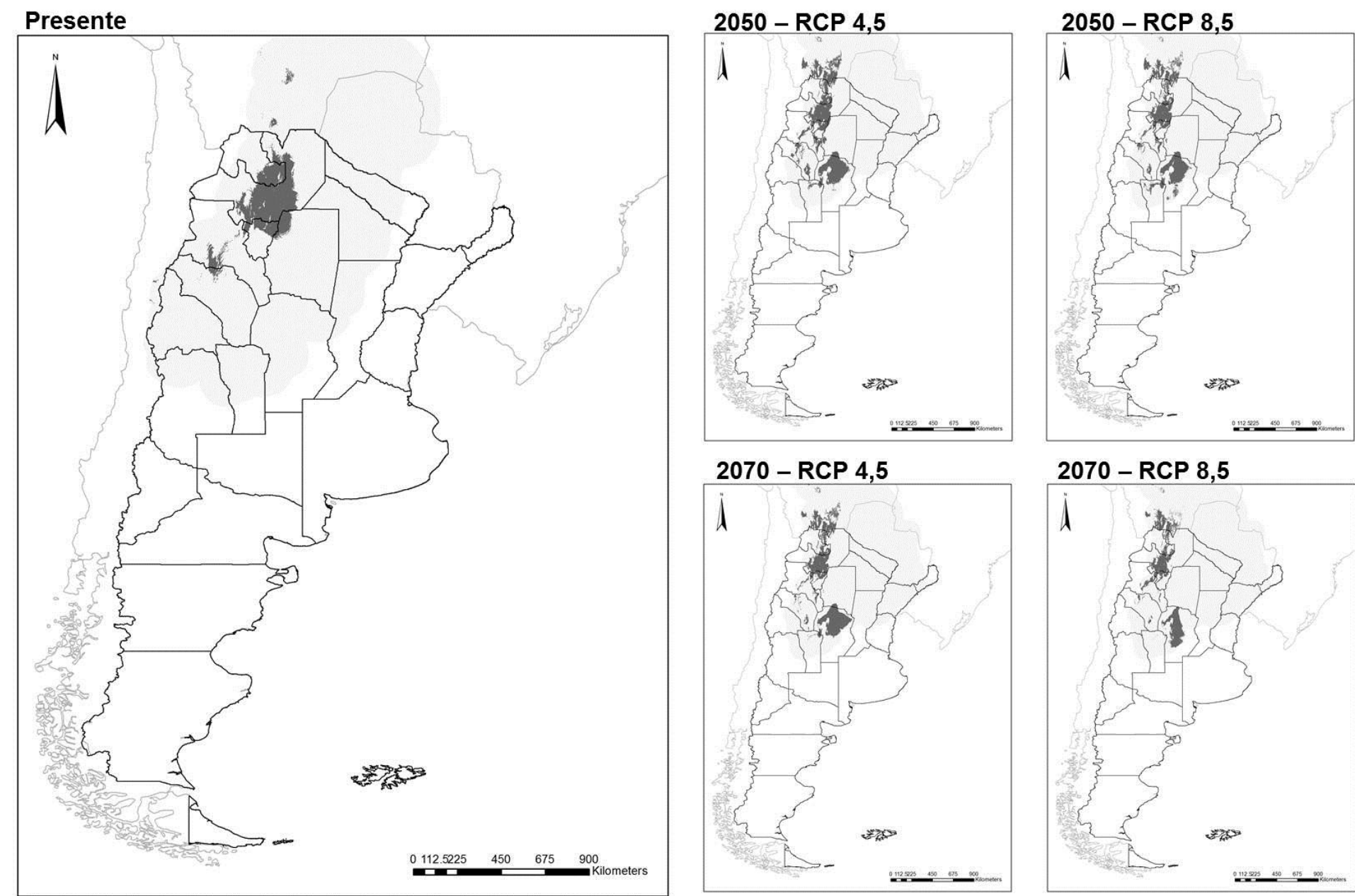

Figura 3. Distribución geográfica potencial en el presente y en el futuro (años 2050 y 2070) bajo diferentes escenarios de cambio climático de la cactácea Cleistocactus smaragdiflorus. 


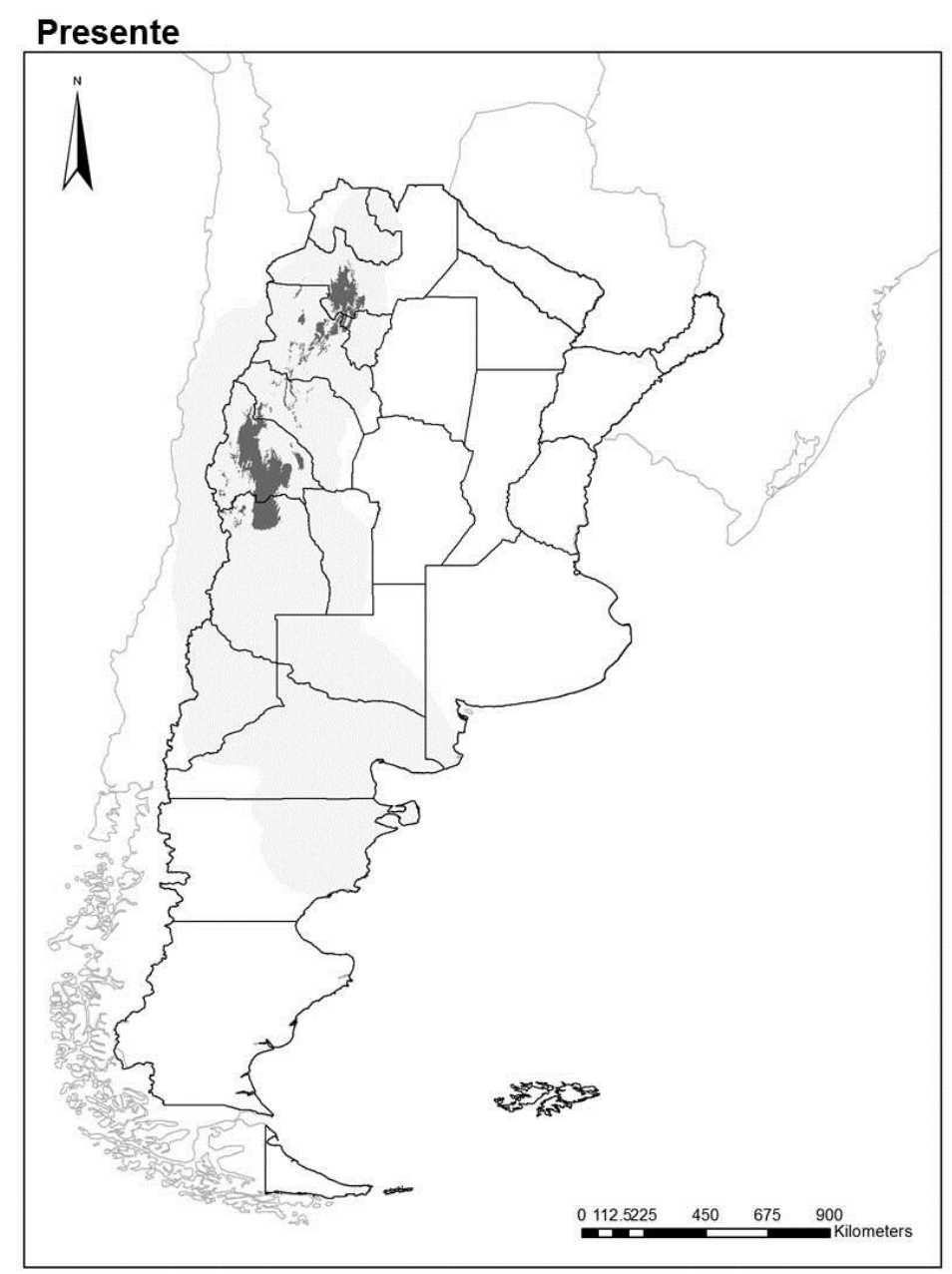

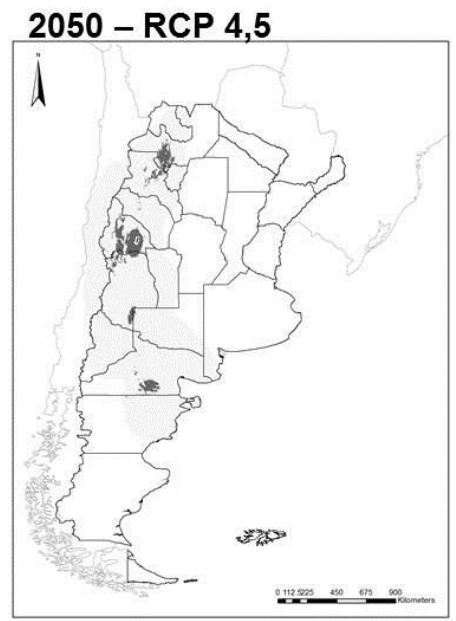

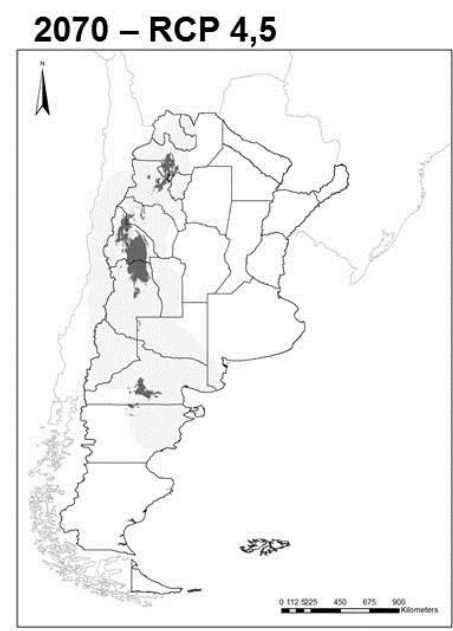

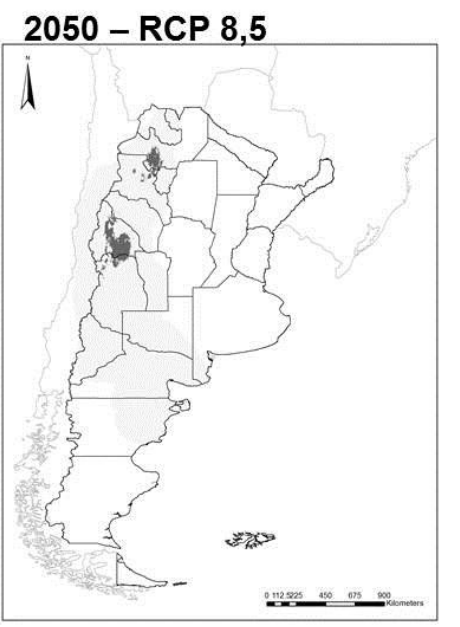

2070 - RCP 8,5

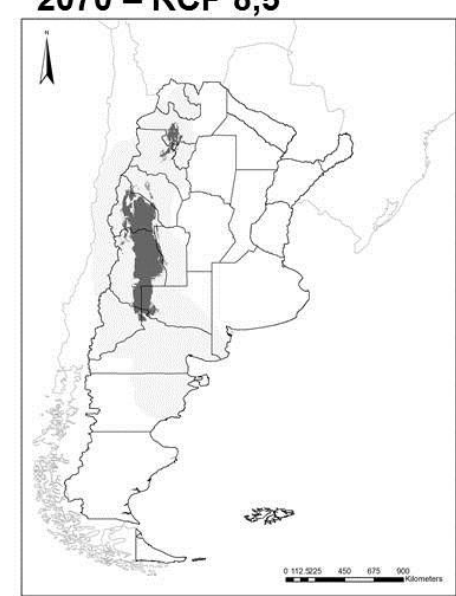

Figura 4. Distribución geográfica potencial en el presente y en el futuro (años 2050 y 2070) bajo diferentes escenarios de cambio climático de la cactácea Denmoza rhodacantha. 


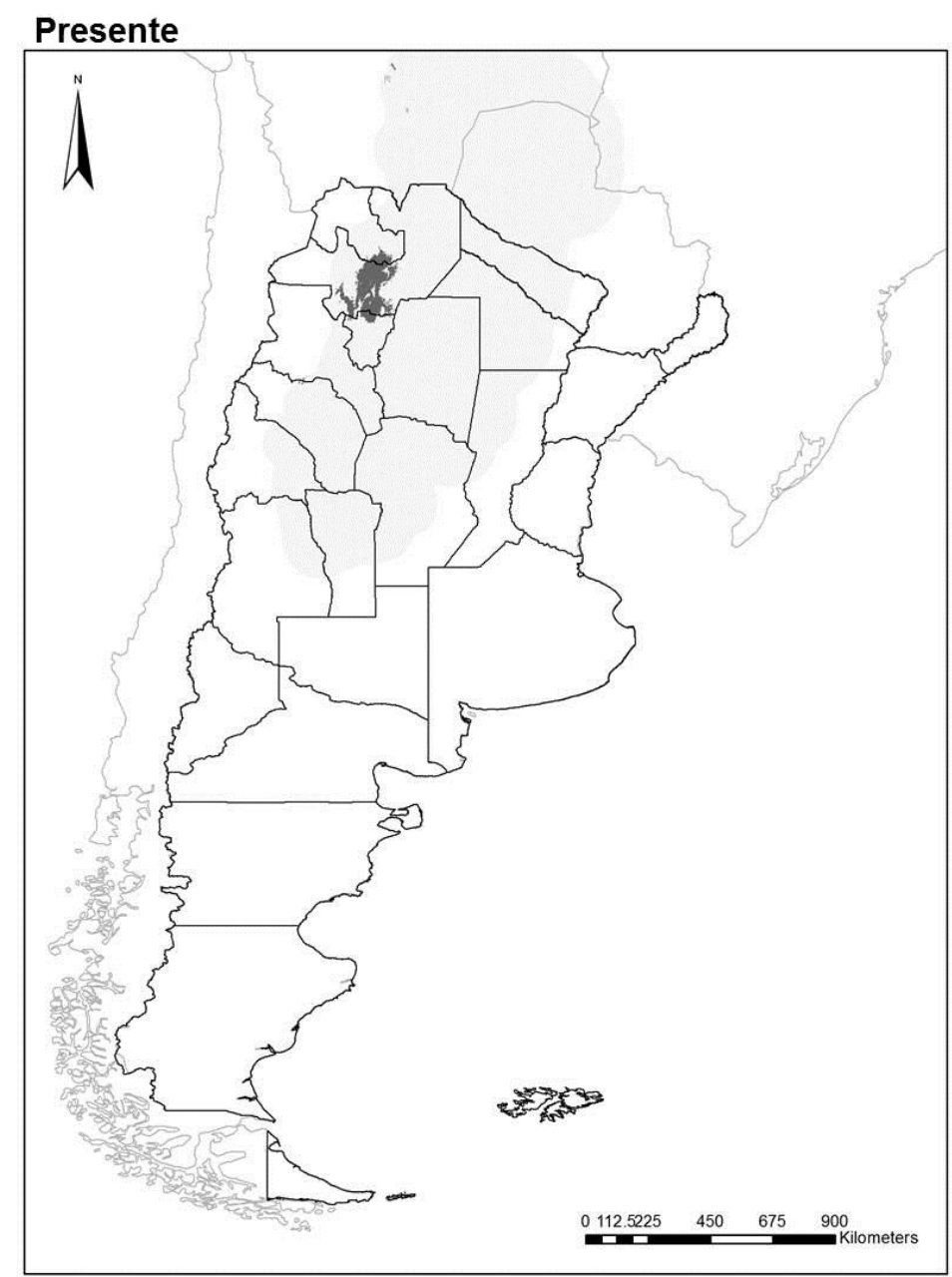

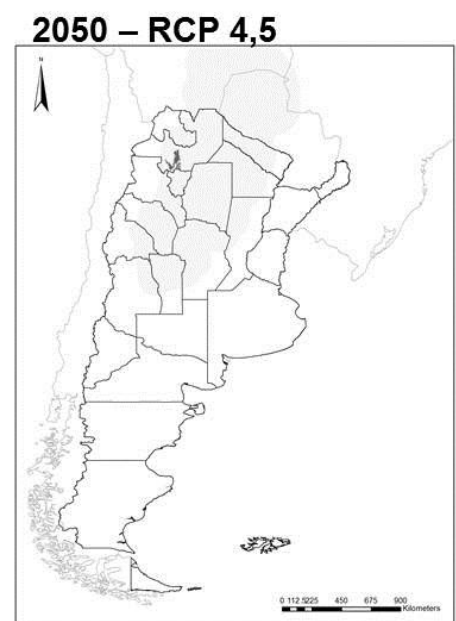
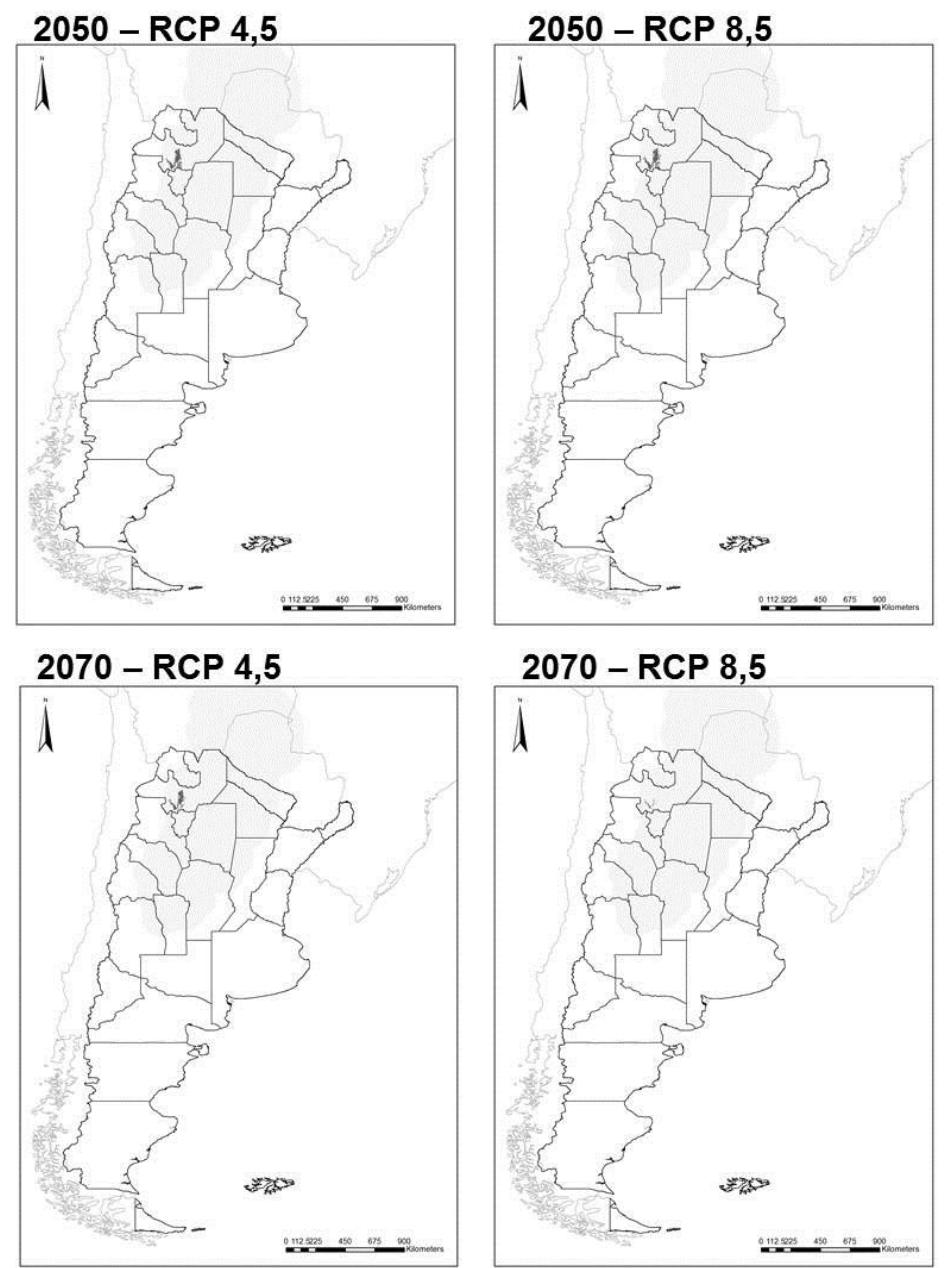

Figura 5. Distribución geográfica potencial en el presente y en el futuro (años 2050 y 2070) bajo diferentes escenarios de cambio climático de la cactácea Echinopsis albispinosa. 

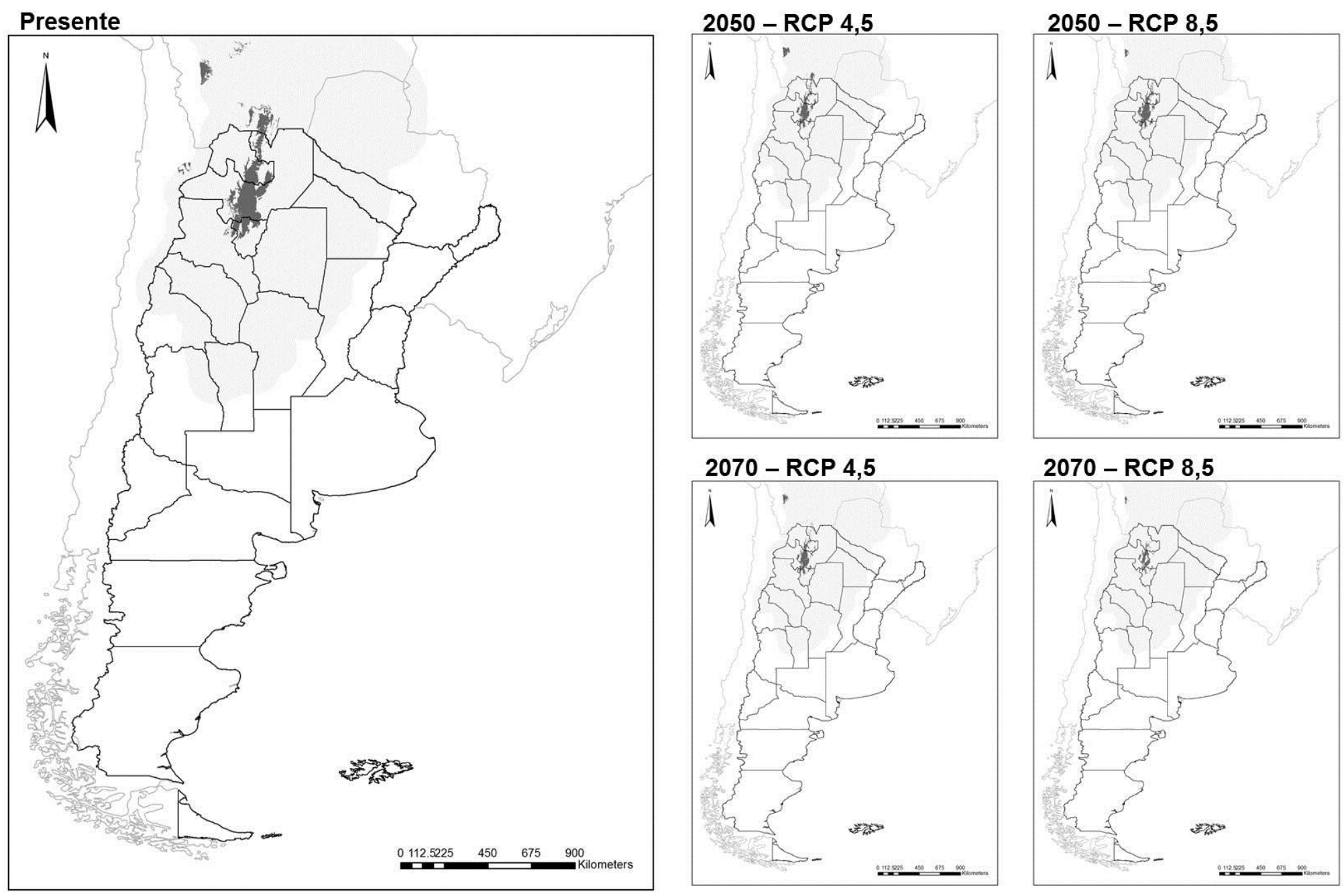

Figura 6. Distribución geográfica potencial en el presente y en el futuro (años 2050 y 2070) bajo diferentes escenarios de cambio climático de la cactácea Echinopsis ancistrophora. 

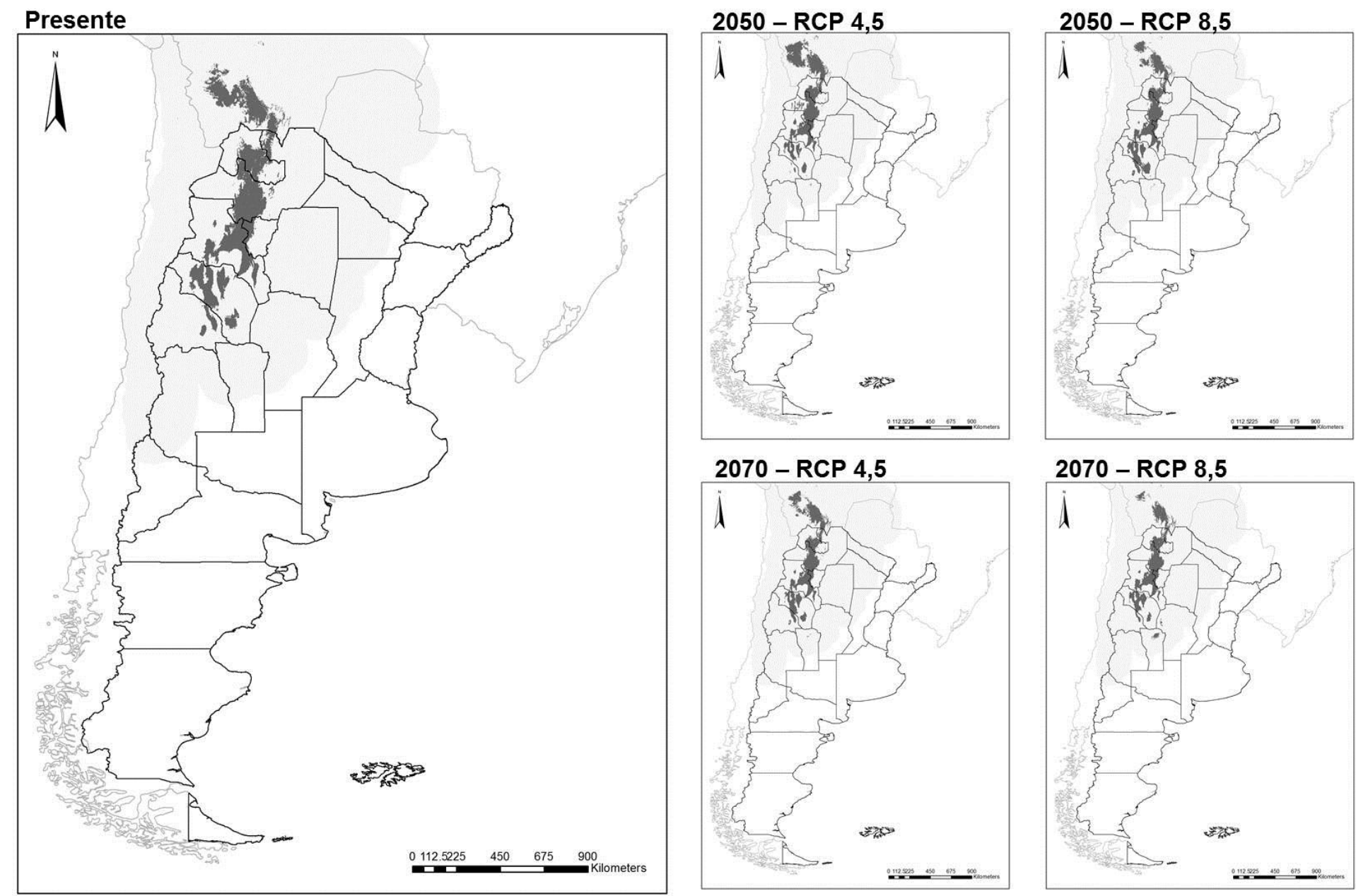

Figura 7. Distribución geográfica potencial en el presente y en el futuro (años 2050 y 2070) bajo diferentes escenarios de cambio climático de la cactácea Echinopsis atacamensis. 

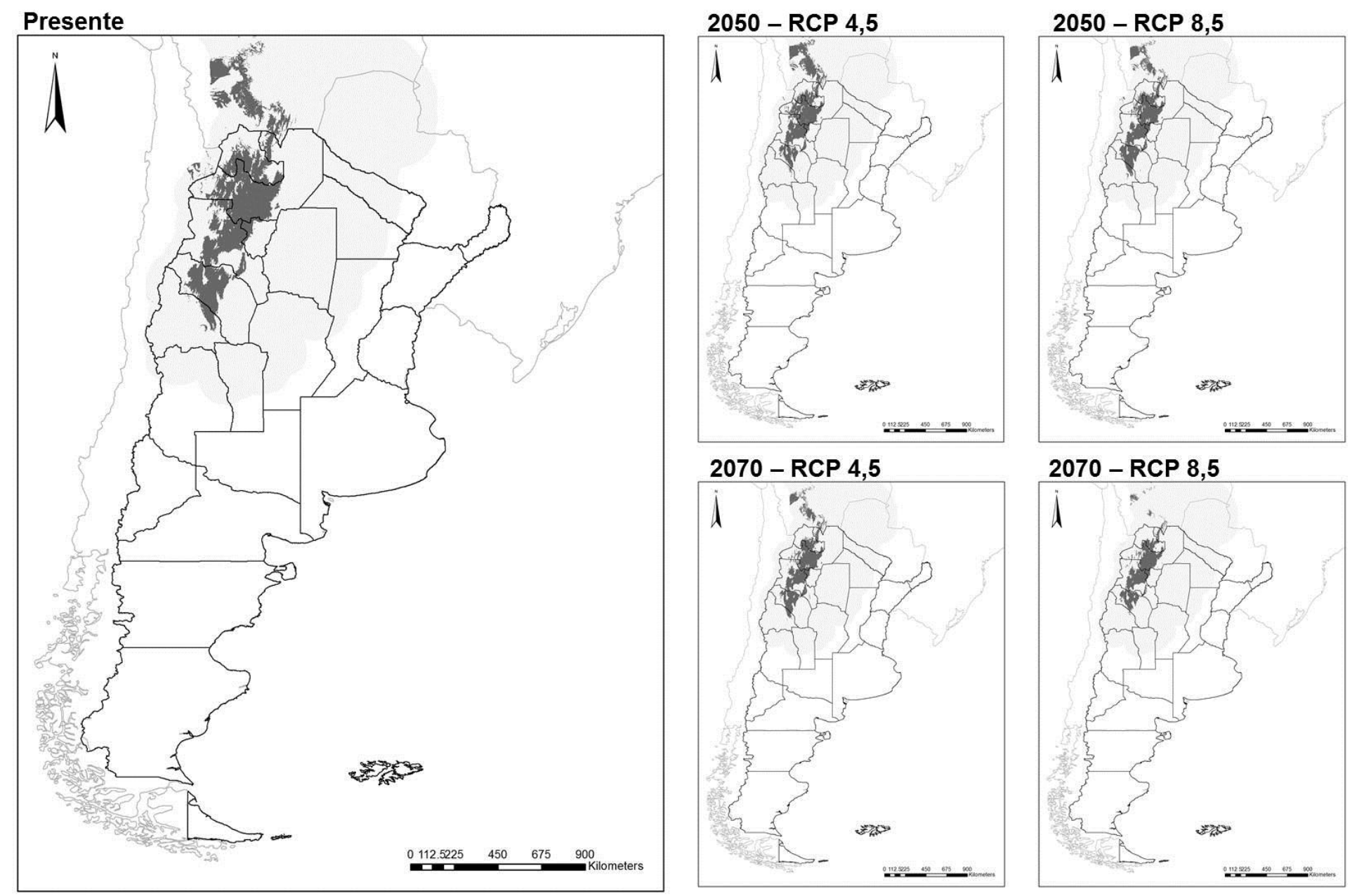

Figura 8. Distribución geográfica potencial en el presente y en el futuro (años 2050 y 2070) bajo diferentes escenarios de cambio climático de la cactácea Echinopsis haematantha. 

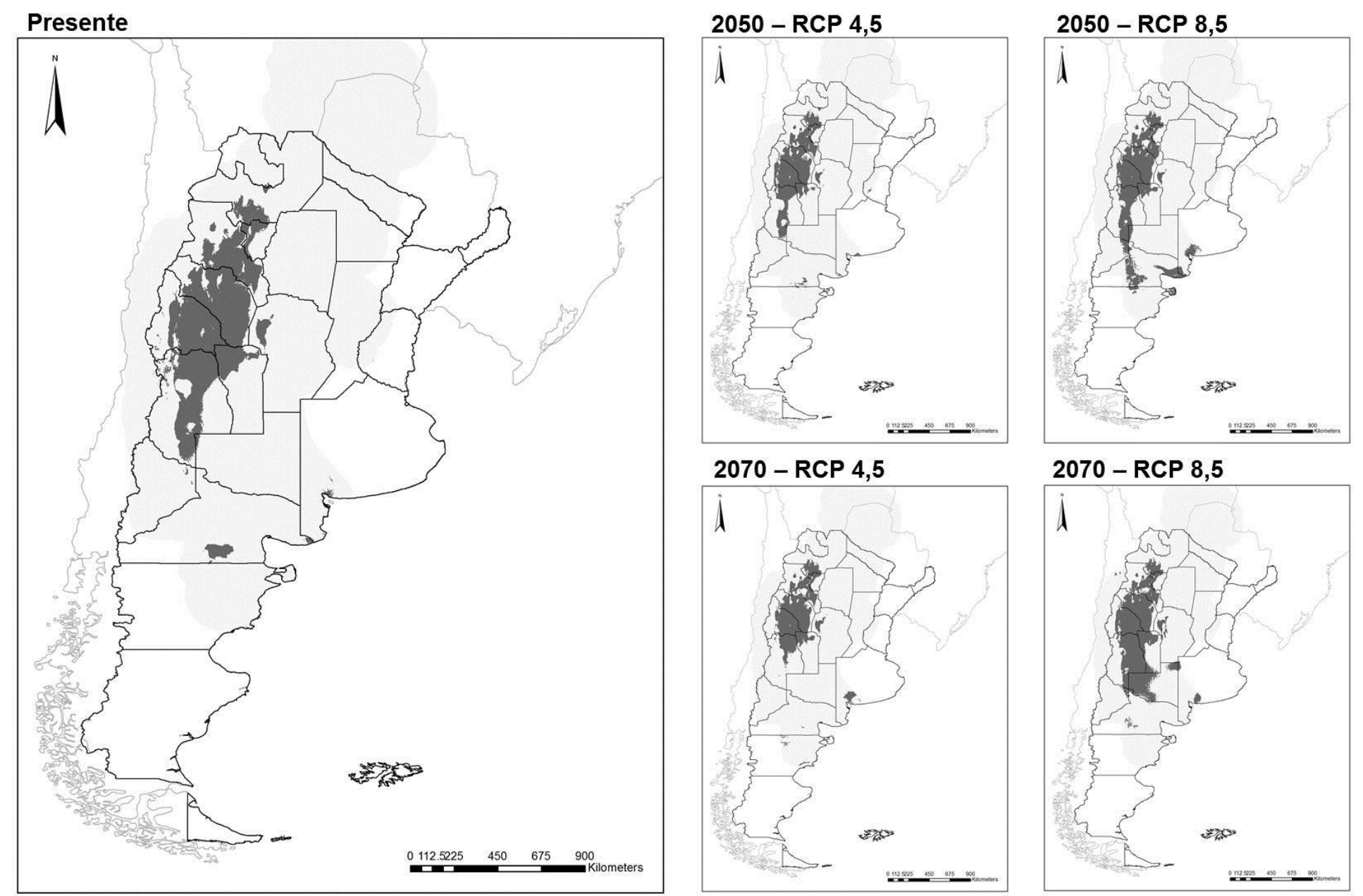

Figura 9. Distribución geográfica potencial en el presente y en el futuro (años 2050 y 2070) bajo diferentes escenarios de cambio climático de la cactácea Echinopsis leucantha. 

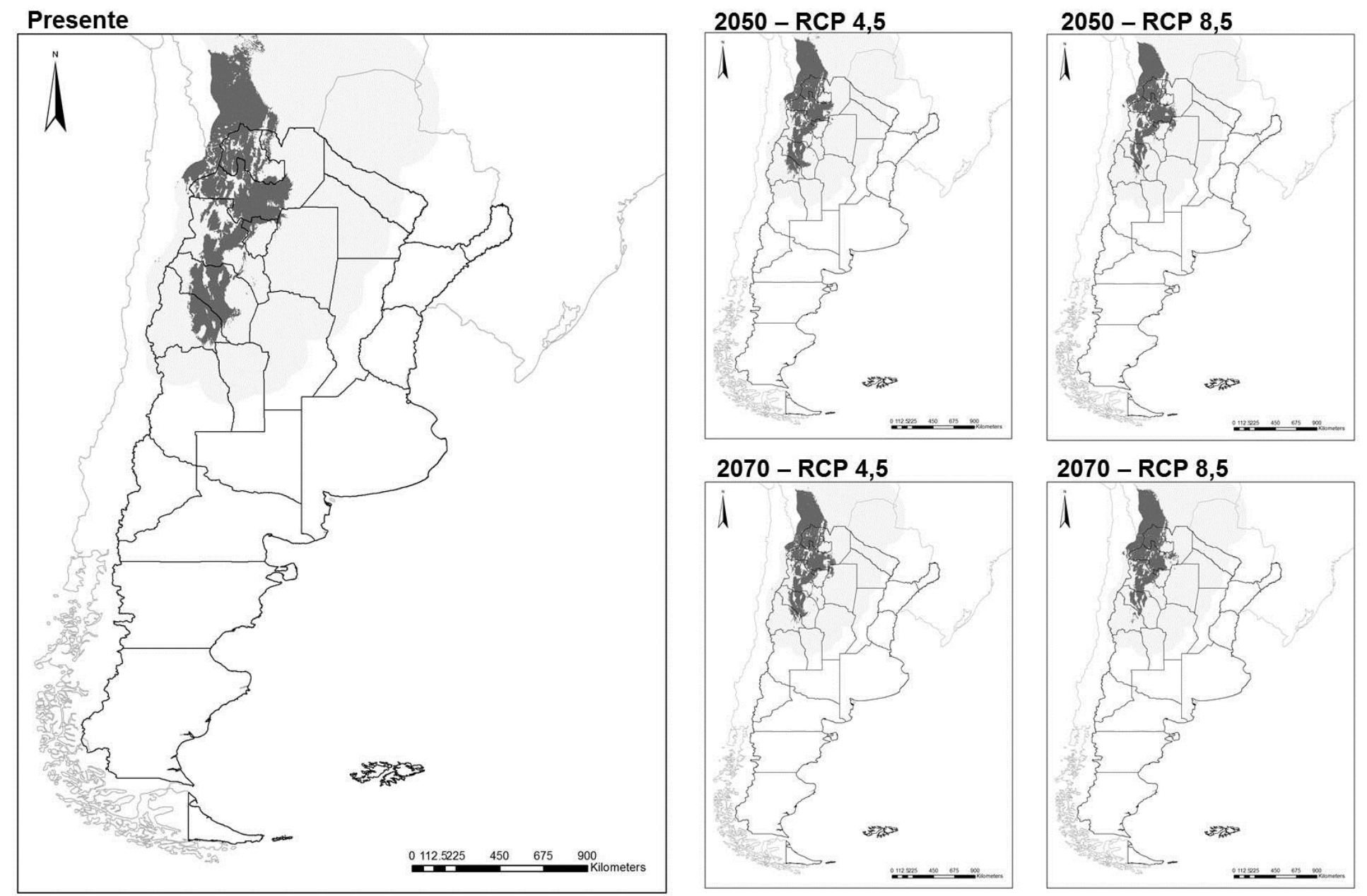

Figura 10. Distribución geográfica potencial en el presente y en el futuro (años 2050 y 2070) bajo diferentes escenarios de cambio climático de la cactácea Echinopsis schickendantzii. 

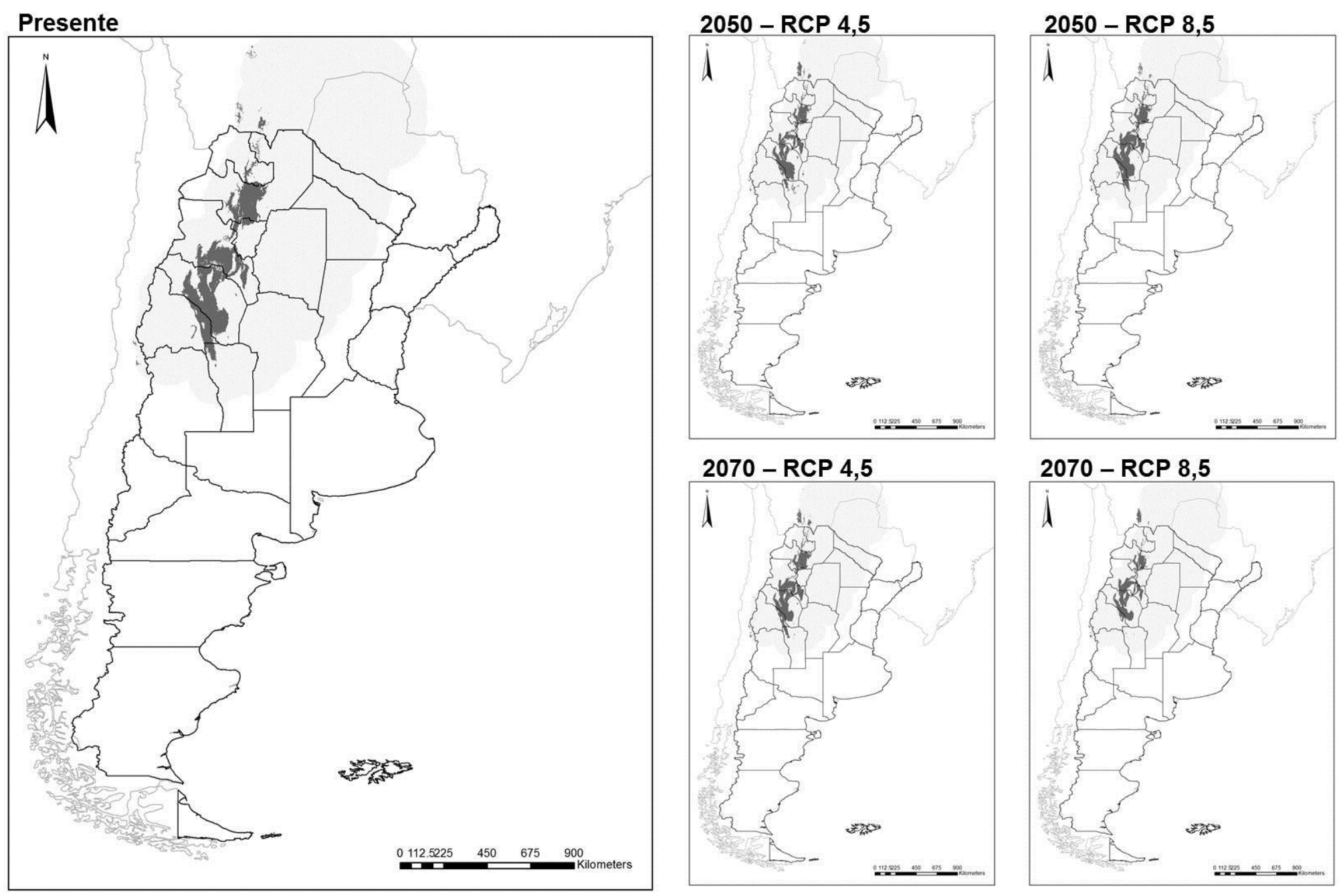

Figura 11. Distribución geográfica potencial en el presente y en el futuro (años 2050 y 2070) bajo diferentes escenarios de cambio climático de la cactácea Echinopsis terscheckii. 

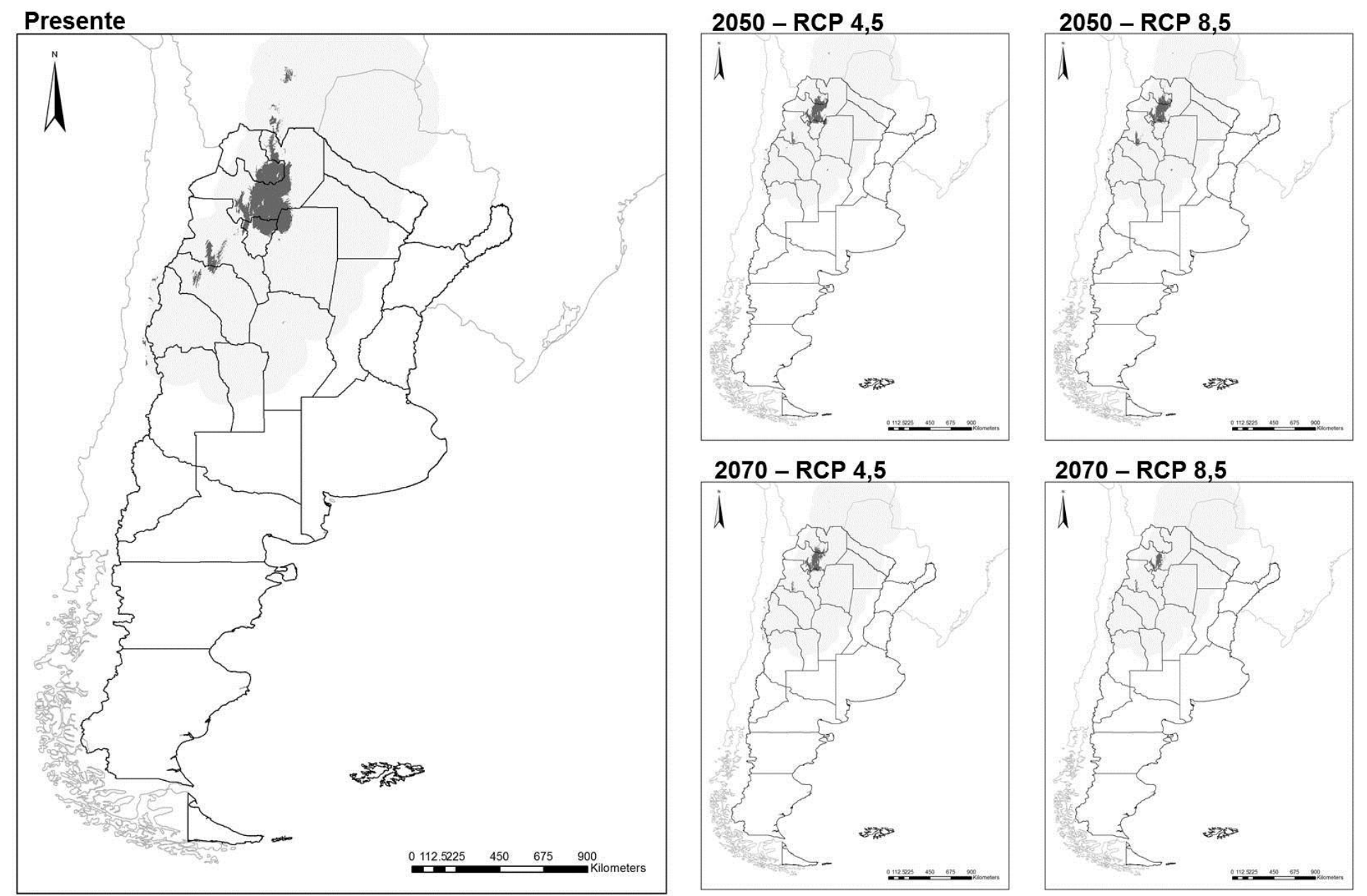

Figura 12. Distribución geográfica potencial en el presente y en el futuro (años 2050 y 2070) bajo diferentes escenarios de cambio climático de la cactácea Echinopsis thelegona. 

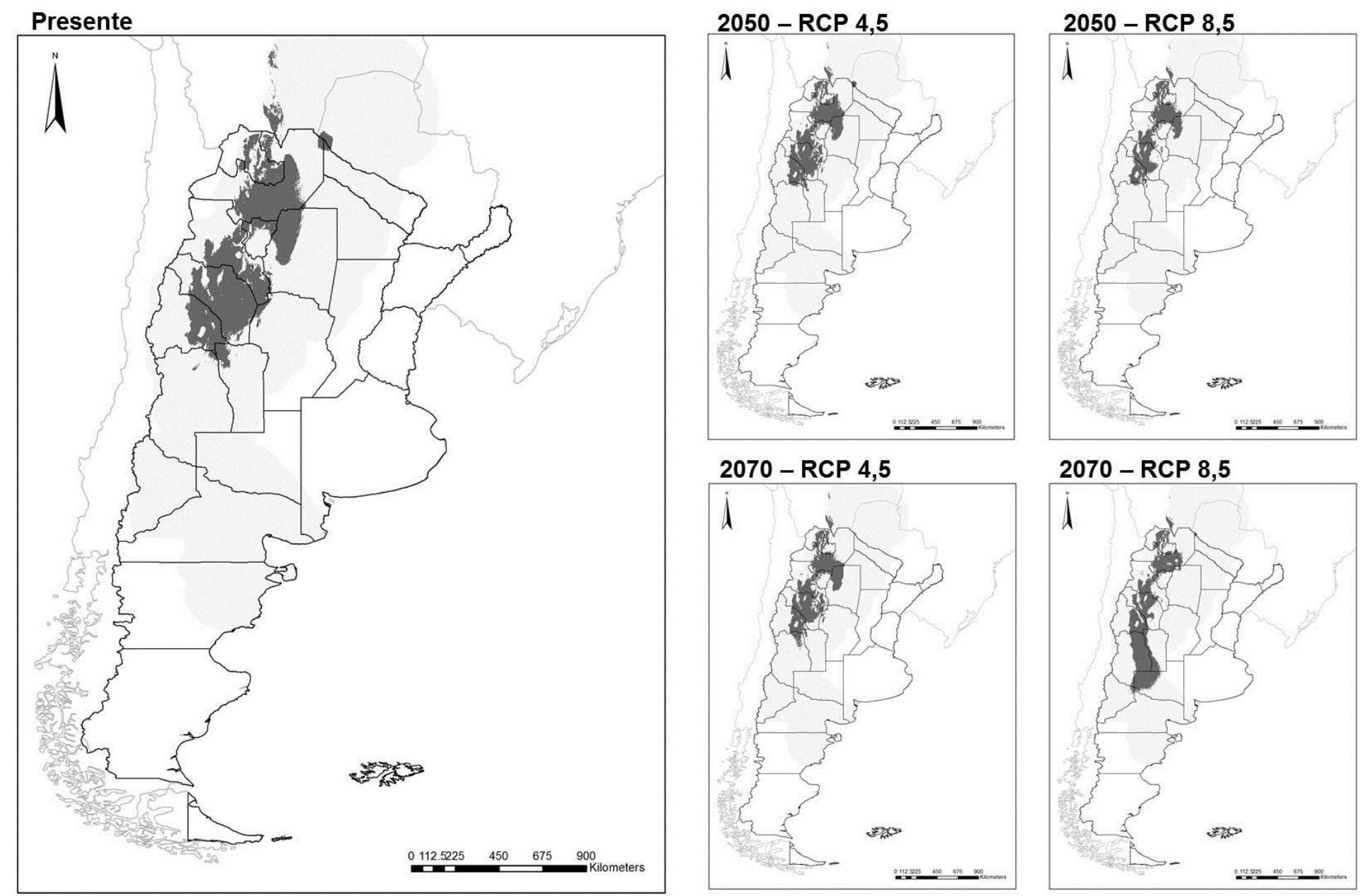

Figura 13. Distribución geográfica potencial en el presente y en el futuro (años 2050 y 2070) bajo diferentes escenarios de cambio climático de la cactácea Gymnocalycium saglionis. 


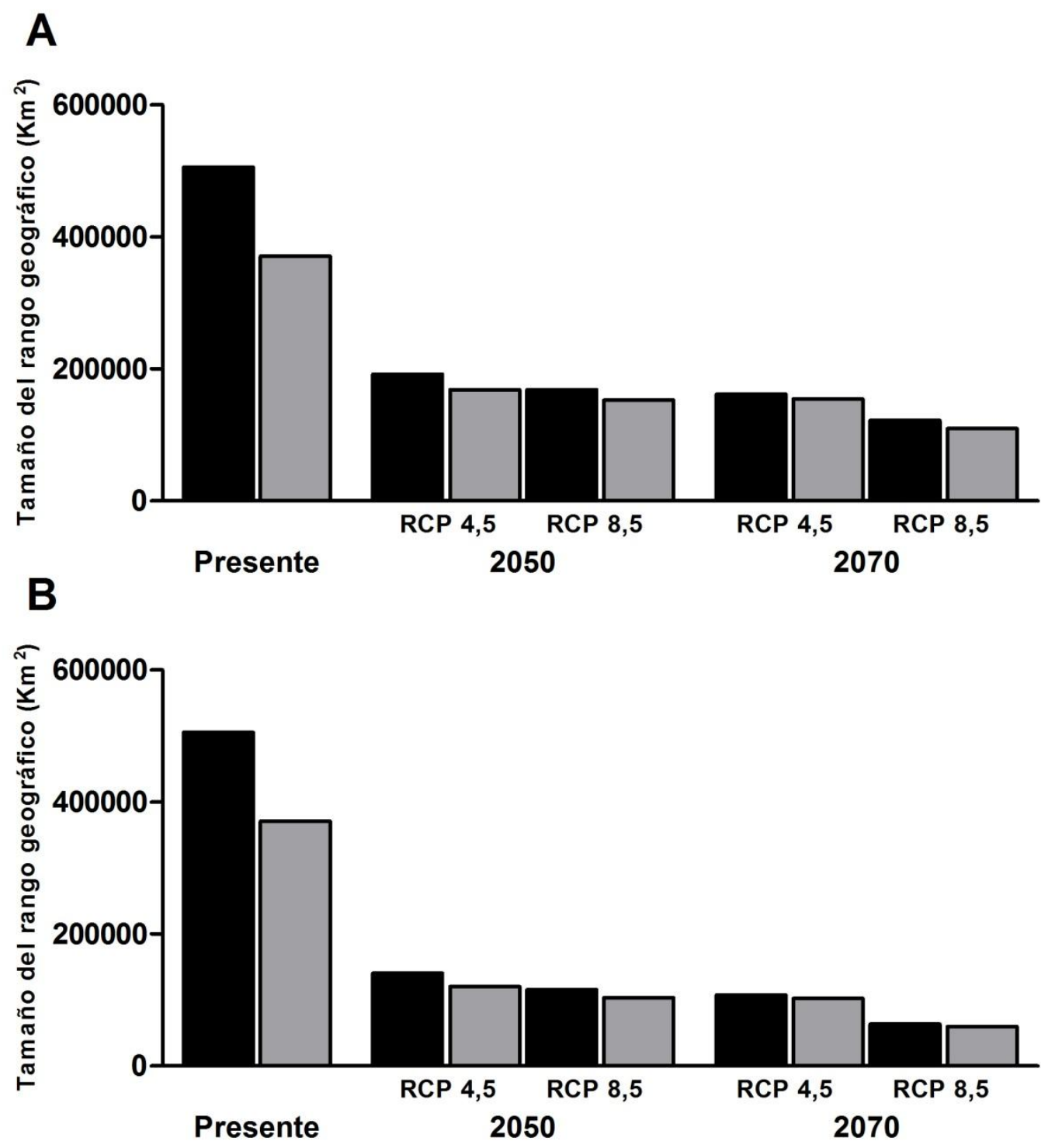

Figura 14. Tamaño del rango geográfico expresado en kilómetros cuadrados para el presente y para el futuro (años 2050 y 2070) bajo diferentes escenarios de cambio climático de la cactácea Cleistocactus baumannii en un escenario con dispersión ilimitada (A) y sin dispersión (B). Las barras negras representan el rango geográfico de la cactácea y las barras grises representan la proporción del rango que comparte con sus polinizadores. 


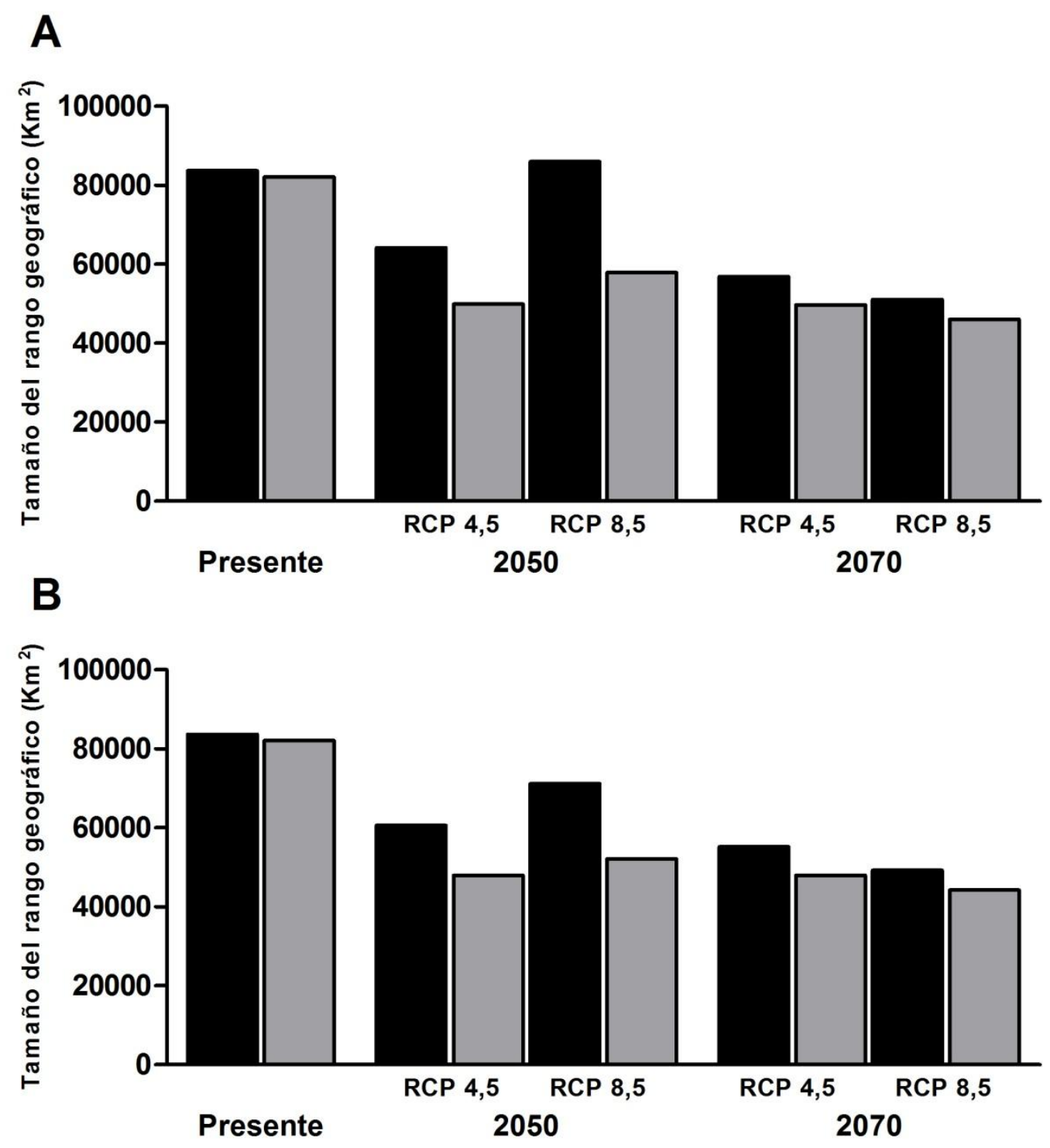

Figura 15. Tamaño del rango geográfico expresado en kilómetros cuadrados para el presente y para el futuro (años 2050 y 2070) bajo diferentes escenarios de cambio climático de la cactácea Cleistocactus smaragdiflorus en un escenario con dispersión ilimitada (A) y sin dispersión (B). Las barras negras representan el rango geográfico de la cactácea y las barras grises representan la proporción del rango que comparte con sus polinizadores. 


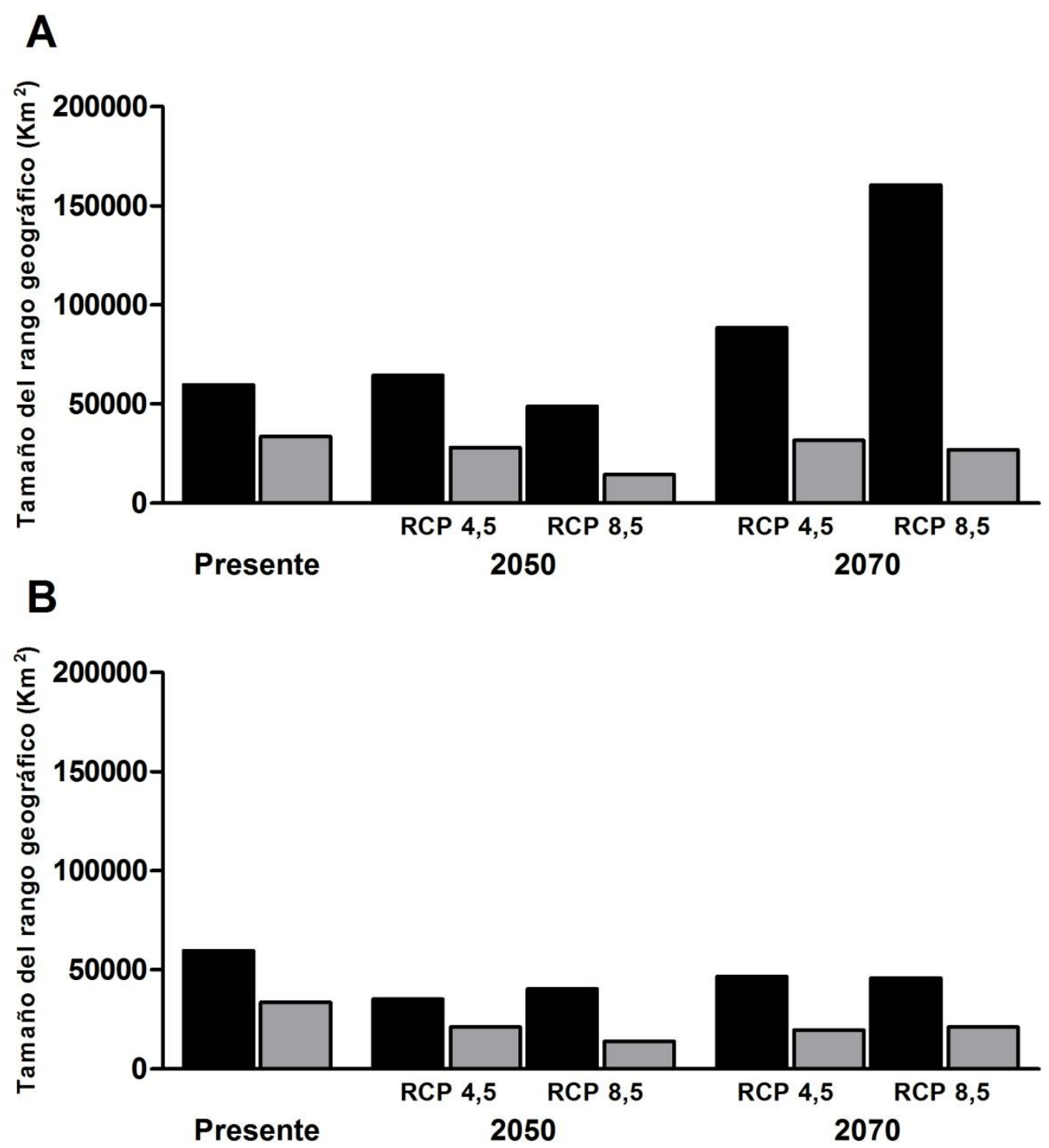

Figura 16. Tamaño del rango geográfico expresado en kilómetros cuadrados para el presente y para el futuro (años 2050 y 2070) bajo diferentes escenarios de cambio climático de la cactácea Denmoza rhodacantha en un escenario con dispersión ilimitada (A) y sin dispersión (B). Las barras negras representan el rango geográfico de la cactácea y las barras grises representan la proporción del rango que comparte con sus polinizadores. 

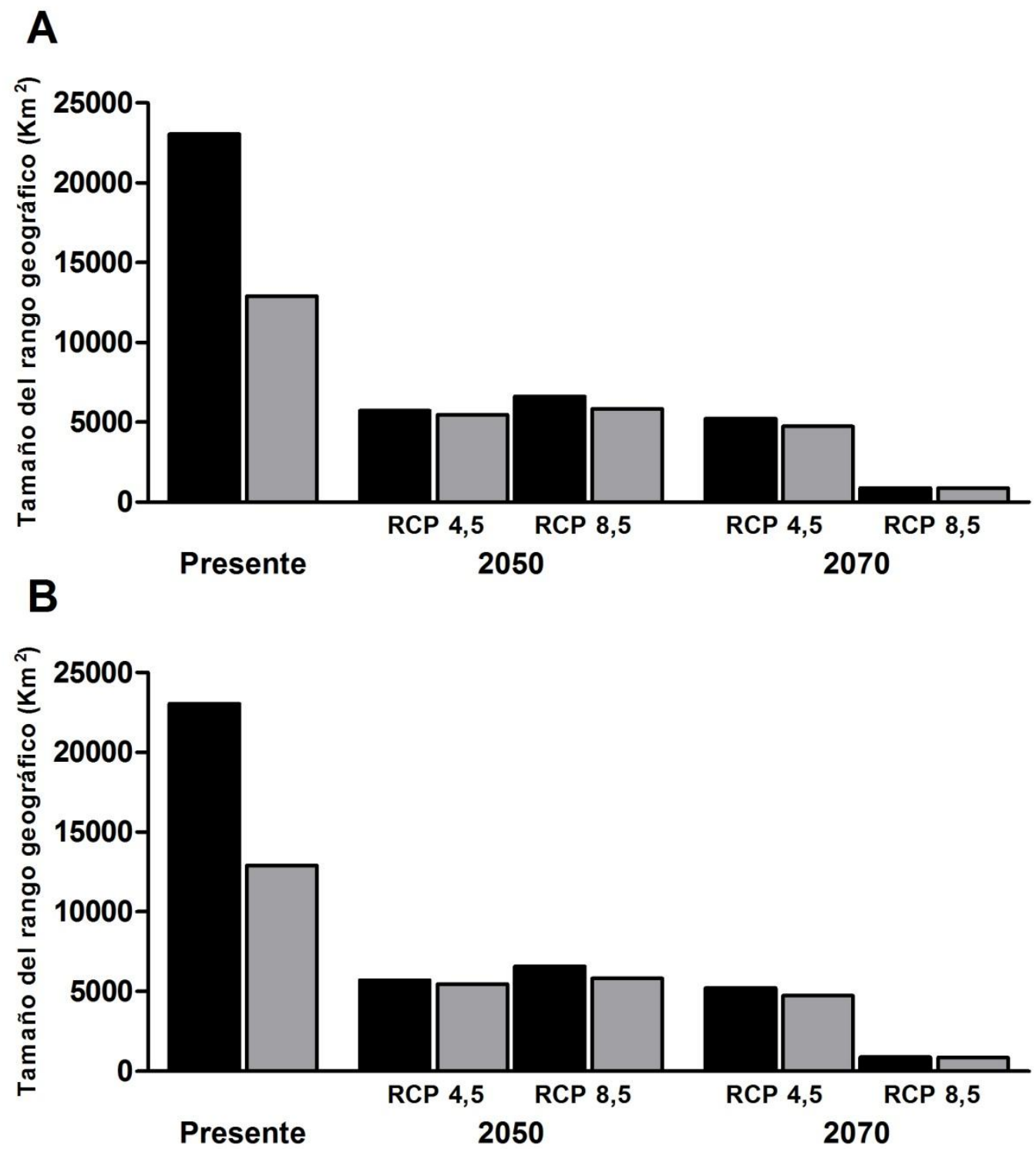

Figura 17. Tamaño del rango geográfico expresado en kilómetros cuadrados para el presente y para el futuro (años 2050 y 2070) bajo diferentes escenarios de cambio climático de la cactácea Echinopsis albispinosa en un escenario con dispersión ilimitada (A) y sin dispersión (B). Las barras negras representan el rango geográfico de la cactácea y las barras grises representan la proporción del rango que comparte con sus polinizadores. 


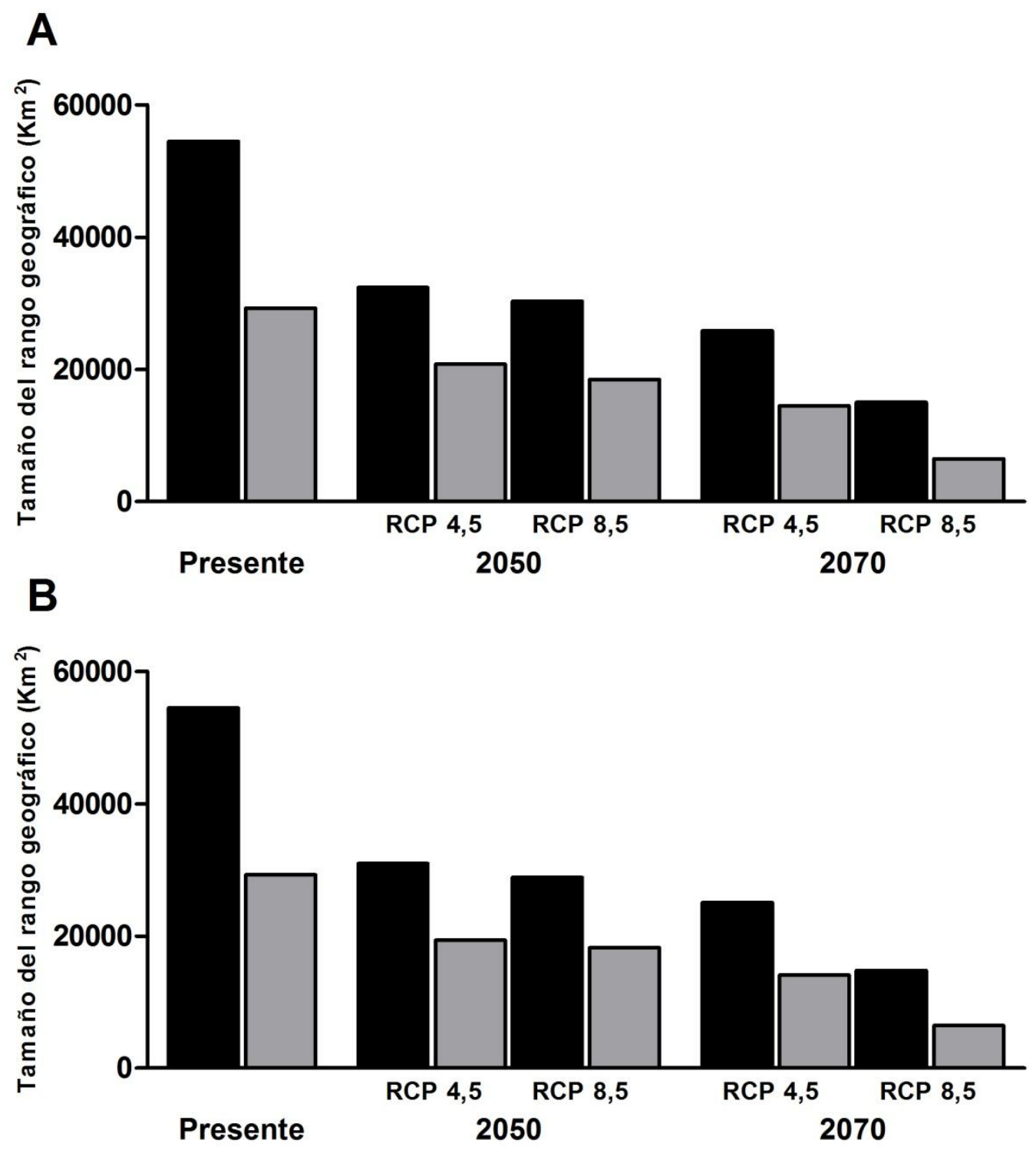

Figura 18. Tamaño del rango geográfico expresado en kilómetros cuadrados para el presente y para el futuro (años 2050 y 2070) bajo diferentes escenarios de cambio climático de la cactácea Echinopsis anistrophora en un escenario con dispersión ilimitada (A) y sin dispersión (B). Las barras negras representan el rango geográfico de la cactácea y las barras grises representan la proporción del rango que comparte con sus polinizadores. 

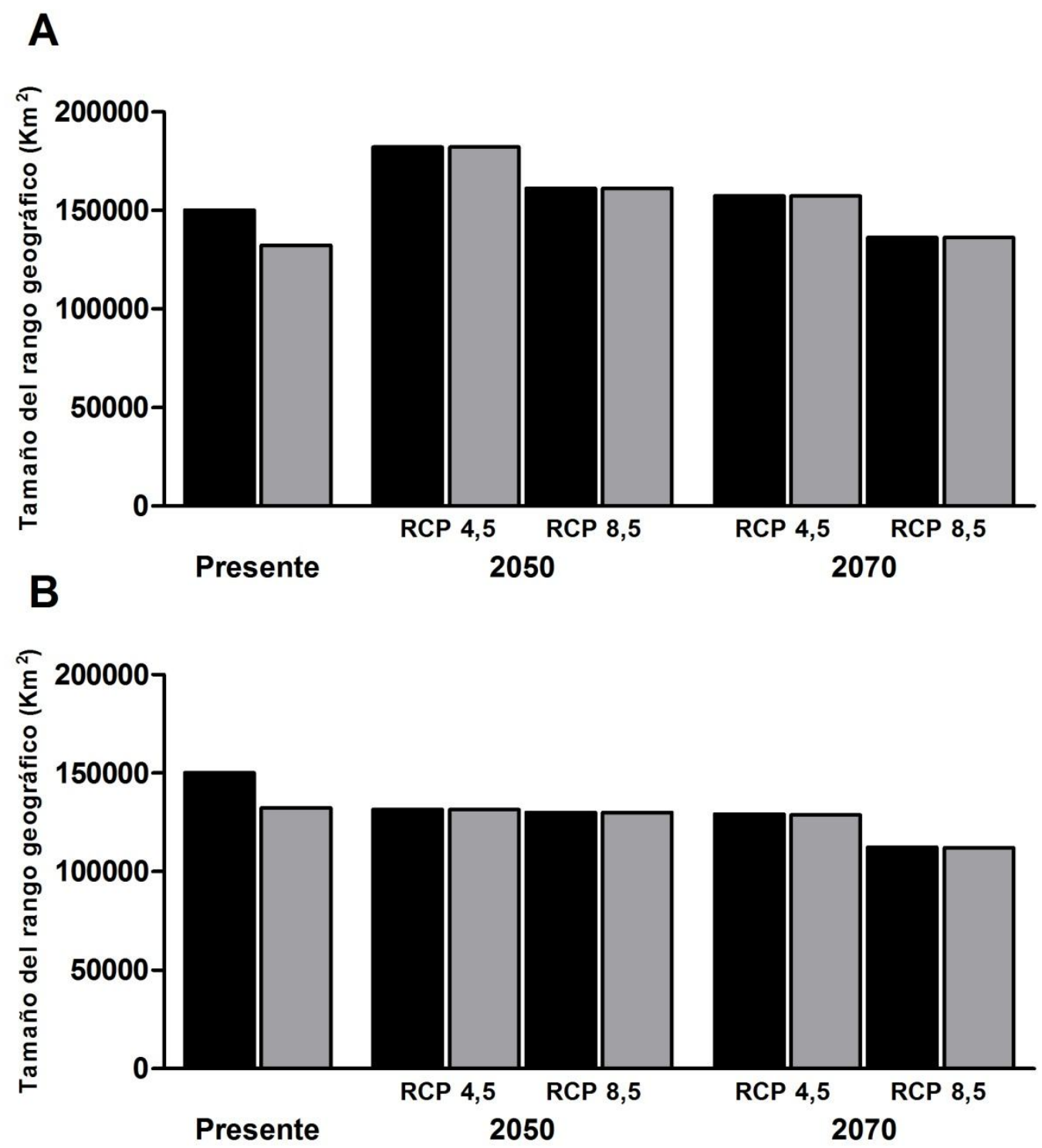

Figura 19. Tamaño del rango geográfico expresado en kilómetros cuadrados para el presente y para el futuro (años 2050 y 2070) bajo diferentes escenarios de cambio climático de la cactácea Echinopsis atacamensis en un escenario con dispersión ilimitada (A) y sin dispersión (B). Las barras negras representan el rango geográfico de la cactácea y las barras grises representan la proporción del rango que comparte con sus polinizadores. 

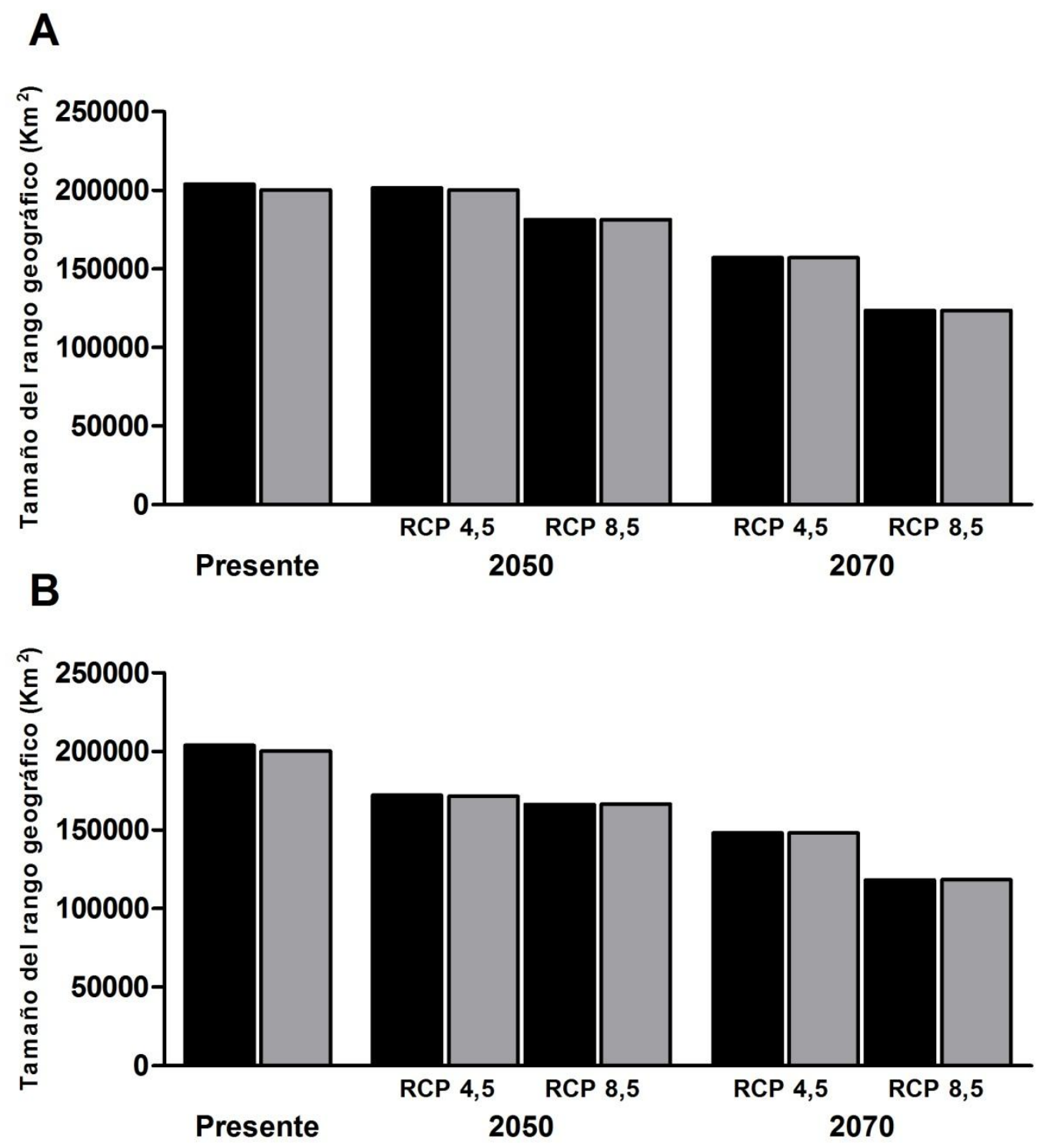

Figura 20. Tamaño del rango geográfico expresado en kilómetros cuadrados para el presente y para el futuro (años 2050 y 2070) bajo diferentes escenarios de cambio climático de la cactácea Echinopsis haematantha en un escenario con dispersión ilimitada (A) y sin dispersión (B). Las barras negras representan el rango geográfico de la cactácea y las barras grises representan la proporción del rango que comparte con sus polinizadores. 

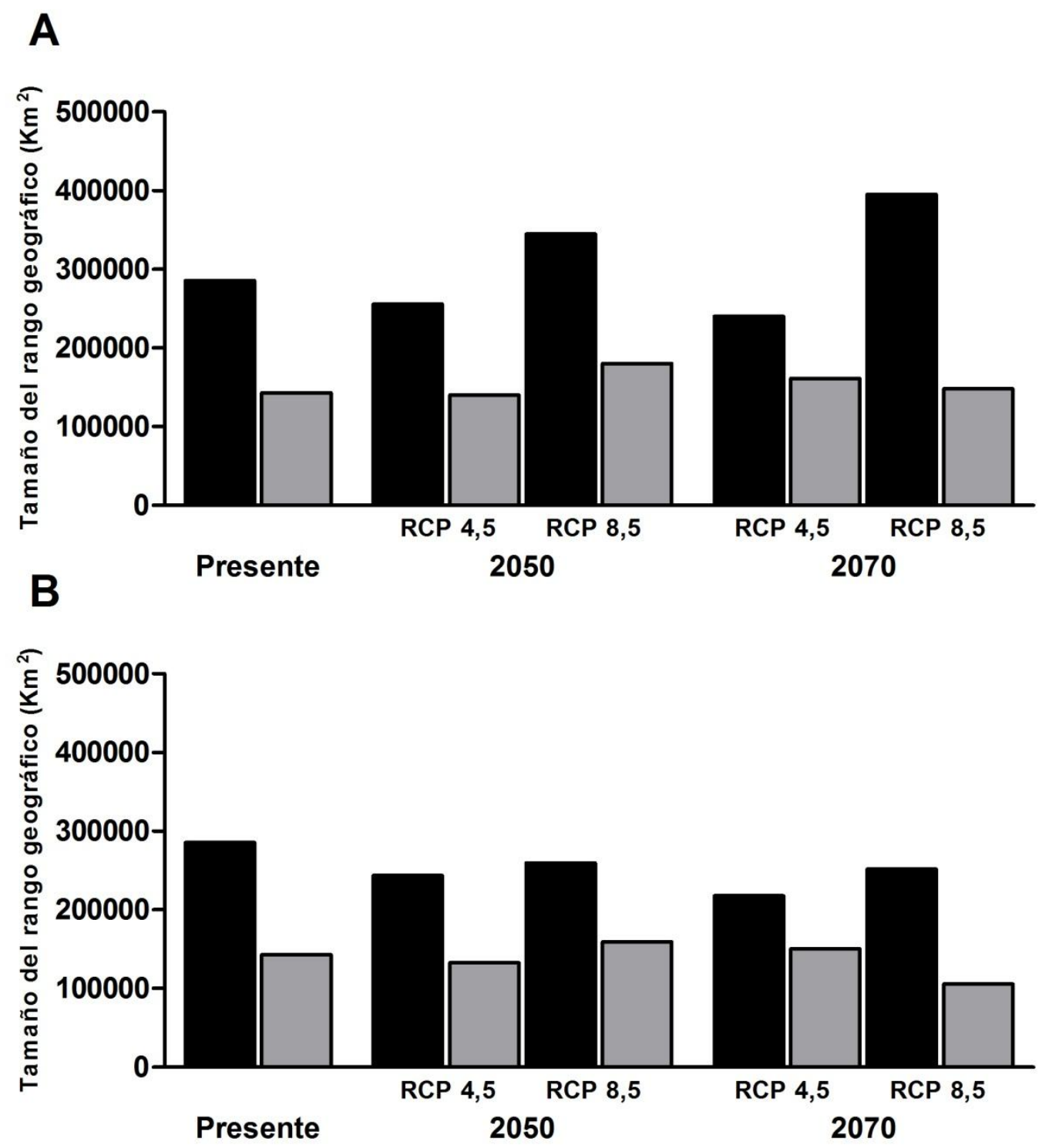

Figura 21. Tamaño del rango geográfico expresado en kilómetros cuadrados para el presente y para el futuro (años 2050 y 2070) bajo diferentes escenarios de cambio climático de la cactácea Echinopsis leucantha en un escenario con dispersión ilimitada (A) y sin dispersión (B). Las barras negras representan el rango geográfico de la cactácea y las barras grises representan la proporción del rango que comparte con sus polinizadores. 

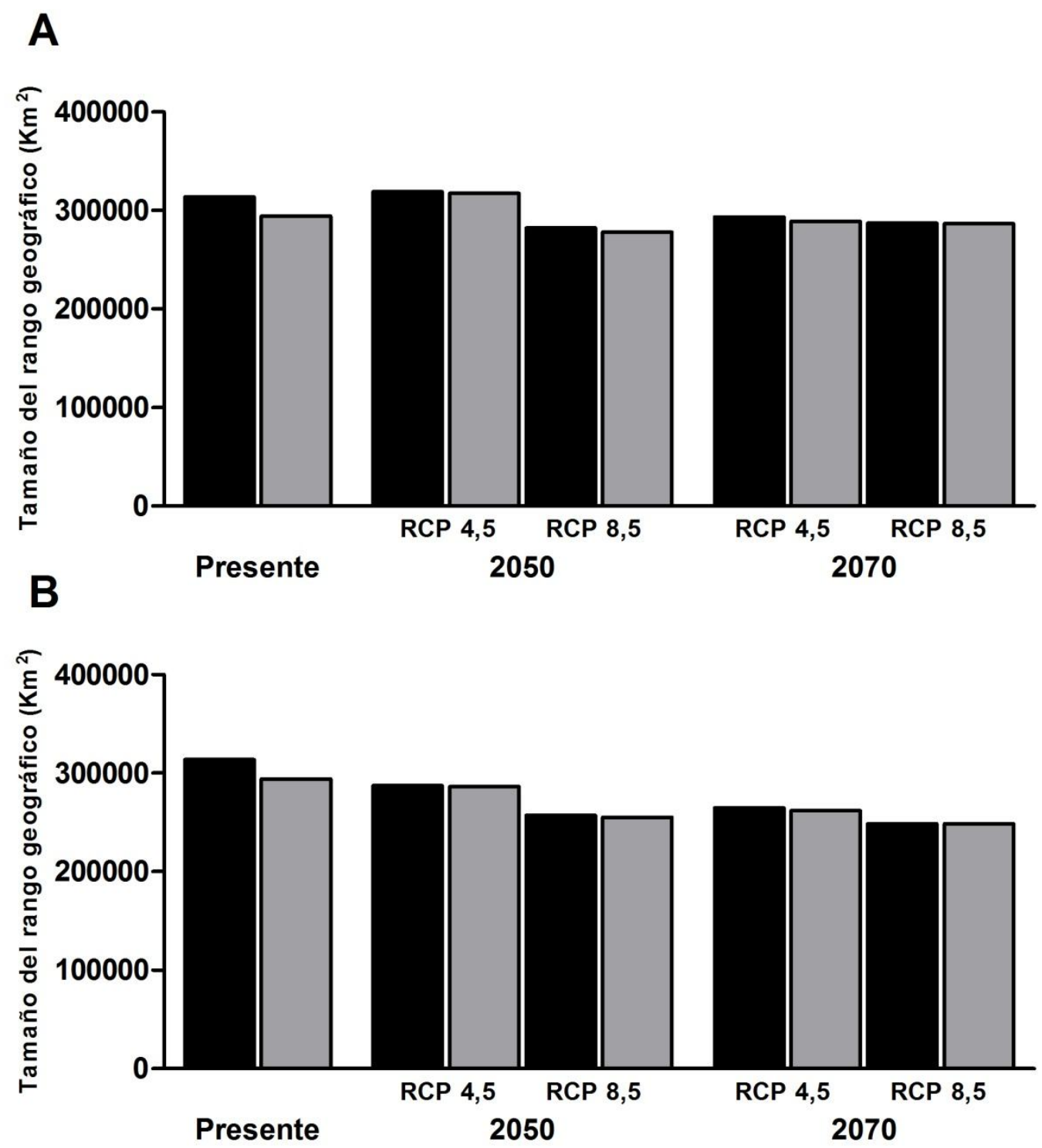

Figura 22. Tamaño del rango geográfico expresado en kilómetros cuadrados para el presente y para el futuro (años 2050 y 2070) bajo diferentes escenarios de cambio climático de la cactácea Echinopsis schickendantzii en un escenario con dispersión ilimitada (A) y sin dispersión (B). Las barras negras representan el rango geográfico de la cactácea y las barras grises representan la proporción del rango que comparte con sus polinizadores. 

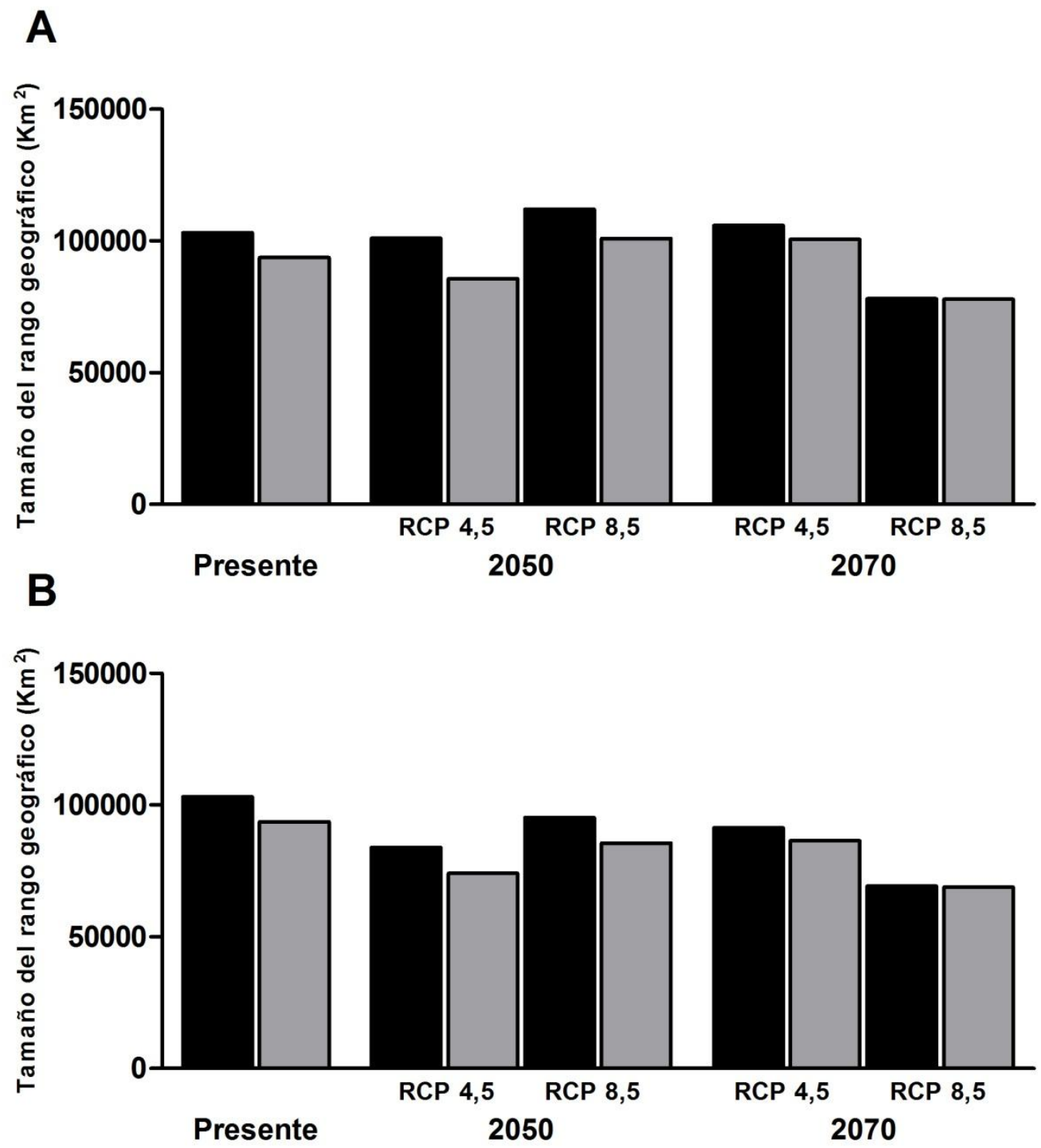

Figura 23. Tamaño del rango geográfico expresado en kilómetros cuadrados para el presente y para el futuro (años 2050 y 2070) bajo diferentes escenarios de cambio climático de la cactácea Echinopsis terscheckii en un escenario con dispersión ilimitada (A) y sin dispersión (B). Las barras negras representan el rango geográfico de la cactácea y las barras grises representan la proporción del rango que comparte con sus polinizadores. 

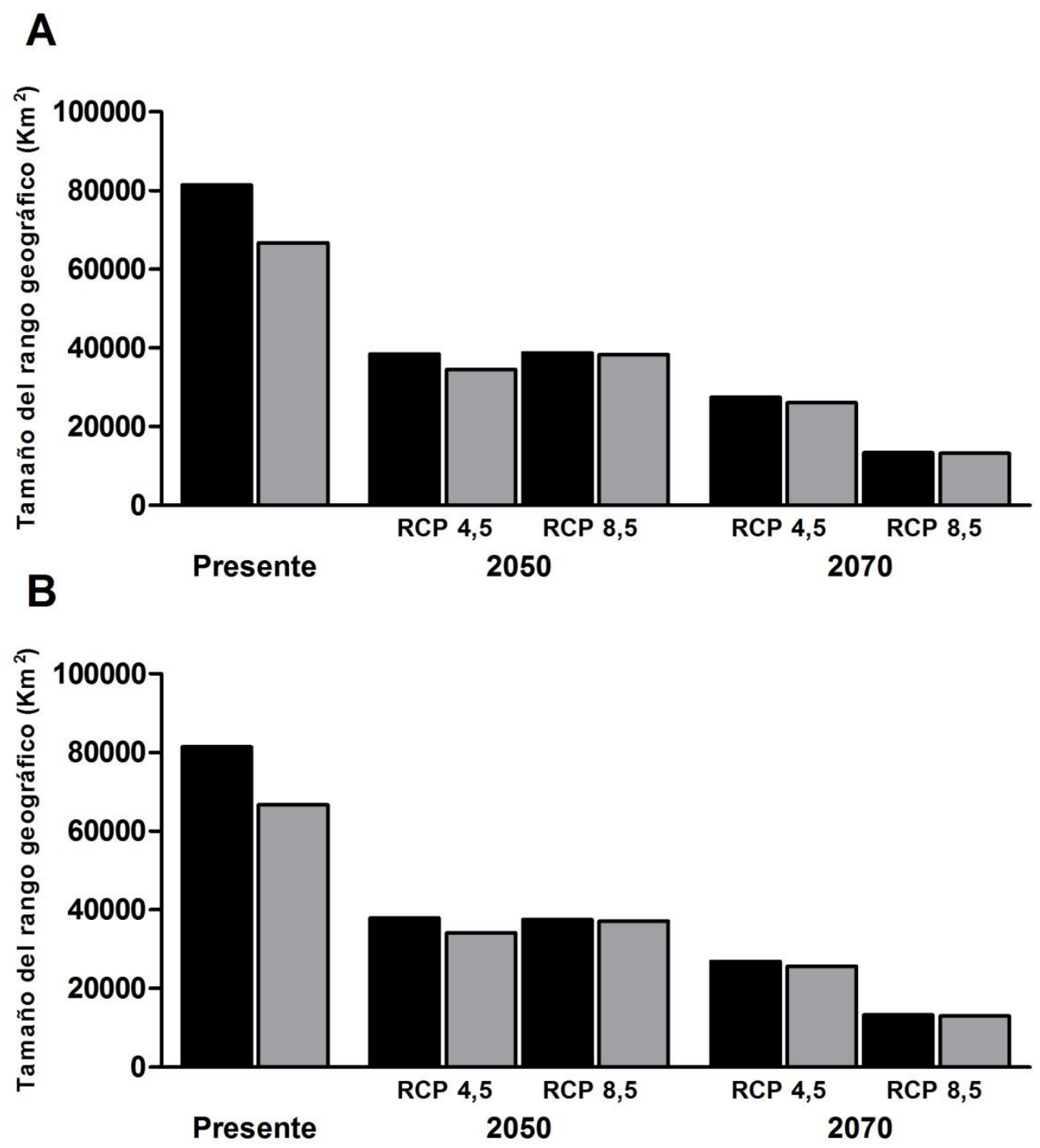

Figura 24. Tamaño del rango geográfico expresado en kilómetros cuadrados para el presente y para el futuro (años 2050 y 2070) bajo diferentes escenarios de cambio climático de la cactácea Echinopsis thelegona en un escenario con dispersión ilimitada (A) y sin dispersión (B). Las barras negras representan el rango geográfico de la cactácea y las barras grises representan la proporción del rango que comparte con sus polinizadores. 

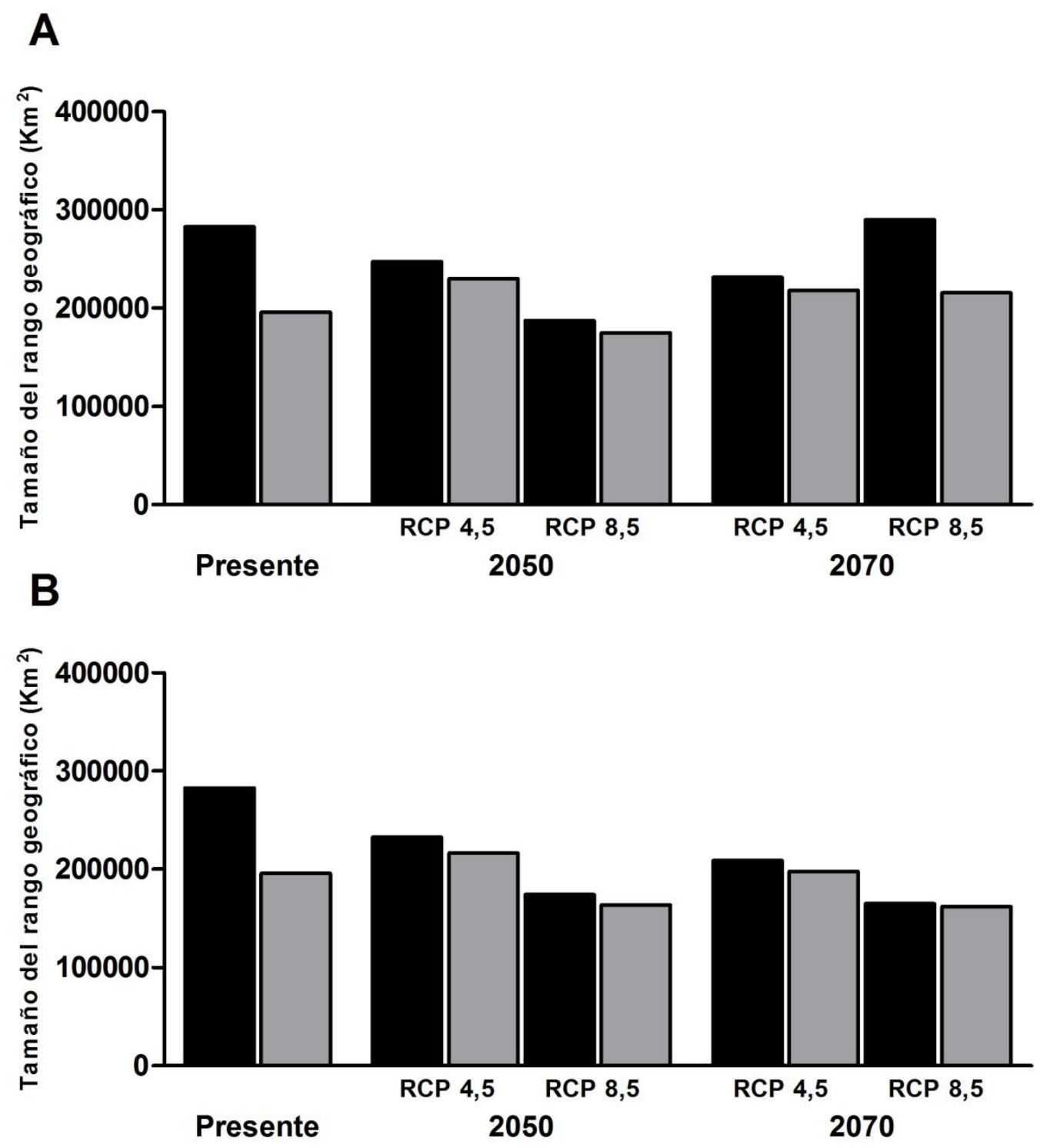

Figura 25. Tamaño del rango geográfico expresado en kilómetros cuadrados para el presente y para el futuro (años 2050 y 2070) bajo diferentes escenarios de cambio climático de la cactácea Gymnocalycium saglionis en un escenario con dispersión ilimitada (A) y sin dispersión (B). Las barras negras representan el rango geográfico de la cactácea y las barras grises representan la proporción del rango que comparte con sus polinizadores. 


\section{CAPÍTULO IV}

Estrategias de conservación in situ para 12 especies de cactáceas del Noroeste de Argentina 


\section{INTRODUCCIÓN}

Las actividades humanas han transformado los ambientes naturales, generando una pérdida de biodiversidad a nivel global que continúa incrementando junto con el tamaño de la población humana. Entre los factores de cambios ambientales que produce la actividad del hombre, los más importantes son la pérdida de hábitat debido a su degradación y fragmentación, la contaminación, las invasiones de especies exóticas, la sobreexplotación, y el cambio climático (Primack 2008, Sodhi 2010). Dada la magnitud de los cambios que generan estos factores sobre los ambientes naturales, se considera que la Tierra se encuentra actualmente en un proceso de cambio global sin precedentes que amenaza a la biodiversidad, provocando la extinción de especies a una tasa equivalente a las experimentadas durante las grandes extinciones masivas que ocurrieron a lo largo de la historia de la vida en el planeta (Primack 2008, Barnosky et al. 2011, IPCC 2014). Por ejemplo, a nivel específico los rangos geográficos de las especies se han restringido y las poblaciones de muchas de ellas han declinado en número; por lo tanto, algunas especies se han extinguido globalmente y otras lo han hecho a escala local (Ceballos y Ehrlich 2002, Primack 2008) Debido a esto, es necesario establecer estrategias tanto in situ como ex situ para la conservación de la biodiversidad a largo plazo.

La diversidad biológica no se distribuye homogéneamente sobre la Tierra (Gaston 2000), y por lo tanto, el patrón de la distribución espacial de dicha diversidad debe ser contemplado al planificar estrategias de conservación. Las áreas que poseen mayores niveles de diversidad (ya sea a nivel de riqueza de especies, de endemismos o de rareza) se consideran como prioritarias para acciones de conservación in situ (Caldecott et al. 1996, Myers 2001). Sin embargo, muchas de las áreas protegidas que existen alrededor del mundo no han sido creadas teniendo en cuenta este principio. Por ejemplo, muchos parques y reservas cuyo objetivo principal es la conservación de un ambiente se han seleccionado con base en condiciones políticas, sociales, recreativas o por potencial turístico entre otras, sin tener en cuenta su aporte a la conservación en un esquema más amplio (Pressey 1994). Debido a esto, mucha de la diversidad existente no estaría representada dentro de las áreas protegidas existentes, y 
sería necesario ampliar el sistema actual de áreas protegidas para contemplarla (Margules y Pressey 2000, Andelman y Willig 2002, Brooks et al. 2004, Rodrigues et al. 2004a, 2004b). Para lograr una mayor efectividad, la identificación de nuevas áreas prioritarias para la conservación debería realizarse con base en los patrones de biodiversidad, asignando una mayor importancia a aquellas áreas que contribuyen en mayor medida al sistema de áreas protegidas (Rodrigues et al. 2004b). Este criterio constituye la base de la planificación sistemática para la conservación, propuesta por Margules y Pressey (2000), que consiste en seis etapas: 1) identificación del sustituto de la biodiversidad que será utilizado para planificar el sistema de áreas protegidas de una región, 2) identificación de la meta cuantitativa de conservación para la región a planificar, 3) evaluación de la efectividad de las reservas existentes para dar cumplimiento a las metas preestablecidas, 4) identificación de las áreas complementarias a las ya establecidas que son necesarias para alcanzar la meta cuantitativa establecida a priori, 5) implementación de las acciones de conservación en las reservas individuales y 6) mantenimiento de los valores requeridos en las áreas de conservación (Margules y Pressey 2000).

Numerosos estudios han propuesto que el cambio climático tiene la capacidad de modificar la distribución geográfica de las especies, representando una amenaza potencial para la biodiversidad. En muchos casos, se estima que estos cambios significarían una reducción en el rango geográfico que ocupan las especies, y desplazamientos latitudinales (generalmente hacia los polos) y altitudinales (generalmente hacia sitios más altos) del mismo (Parmesan y Yohe 2003, Parmesan 2006, Bellard et al. 2012). Debido a esto, la efectividad de los sistemas de áreas protegidas podría verse alterada bajo escenarios de cambio climático futuro (Araújo et al. 2011), y por lo tanto, las estrategias de conservación deberían tener en cuenta los efectos de este fenómeno sobre los patrones de diversidad.

Las interacciones bióticas son un componente fundamental del nicho ecológico de las especies. Si bien se considera que los factores abióticos (tales como las condiciones climáticas) definen el rango geográfico de una especie en gran medida, se ha propuesto que las interacciones entre especies (por ejemplo, 
la competencia y los mutualismos) también influyen en los límites geográficos de la misma (Araújo y Luoto 2007, Soberón 2007, Wisz et al. 2013). Sin embargo, la identificación de áreas que son prioritarias para la conservación, generalmente se realiza con base en la riqueza de especies estimada a partir del rango geográfico de las especies del grupo de interés, sin tener en cuenta a las especies con las cuales interactúan y que en muchos casos permiten la persistencia de sus poblaciones. Una de las formas de incorporar las interacciones mutualistas a los esquemas de conservación sería a través de la estimación de un rango geográfico acoplado entre las especies; es decir, que incluya sólo a las áreas que las especies interactuantes comparten (Capítulo III). De esta forma, se garantiza que en los sitios que pueden ser seleccionados como prioritarios para la conservación estén presentes tanto la especie focal que se desea conservar, como las especies de las cuales depende para su persistencia.

Las cactáceas son elementos conspicuos de las zonas áridas y semi-áridas que se distribuyen en el continente Americano. Debido, principalmente, al cambio en el uso del suelo y a su valor de uso, esta familia enfrenta amenazas que la convierten en un grupo prioritario para acciones de conservación. Se estima que más del $30 \%$ de las especies de la familia se encuentran en peligro de extinción (Ortega-Baes et al. 2010, Goettsch et al. 2015). El noroeste de Argentina presenta una alta riqueza de especies de esta familia, formando parte del segundo centro más importante de diversidad a nivel global, que se concentra en los Andes centrales (Ortega-Baes et al. 2010, Ortega-Baes et al. 2015). Sin embargo, los estudios acerca de la biología de las especies que viven en esta región, fundamentales para la instrumentación de estrategias de conservación, son aún incipientes (Ortega-Baes et al. 2012, Ortega-Baes et al. 2015, Godoy-Bürki et al. 2016). Las cactáceas establecen interacciones mutualistas con otras especies vinculadas a la polinización, la dispersión de semillas y el establecimiento de nuevos individuos (Pimienta-Barrios y del Castillo 2002, Godínez-Álvarez et al. 2003). Dada la importancia de estas interacciones en el ciclo de vida de las especies de esta familia, es necesario tenerlas en cuenta al establecer estrategias de conservación in situ para este grupo de plantas prioritarias. 
En este contexto, el presente capítulo tuvo como objetivo establecer un esquema de conservación in situ para 12 especies de cactáceas de la tribu Trichocereeae del noroeste de Argentina de las que se tiene conocimiento acerca de su biología de la polinización (Capítulo I), teniendo en cuenta sus patrones de riqueza en la actualidad y en el futuro bajo escenarios de cambio climático, y también el rango geográfico que comparten con sus mutualistas (polinizadores). Se pusieron a prueba las siguientes hipótesis: 1) si el nivel de acople espacial entre las cactáceas y sus polinizadores es alto, habrá un alto nivel de congruencia (es decir, de acople espacial) entre las áreas prioritarias para la conservación seleccionadas a partir de la distribución de las cactáceas con las seleccionadas a partir de la distribución que comparten con sus polinizadores y 2) debido a que el cambio climático afectaría el rango geográfico de las especies, las áreas prioritarias para la conservación de este grupo de especies en el presente tendrán un bajo nivel de congruencia con las áreas prioritarias seleccionadas con base en las distribuciones en el futuro. Los objetivos específicos fueron: a) seleccionar las áreas prioritarias para la conservación de 12 especies de cactáceas teniendo en cuenta su rango de distribución y el rango que comparten con sus polinizadores; b) seleccionar las áreas prioritarias para la conservación de este grupo de especies bajo escenarios de cambio climático futuro, teniendo en cuenta el rango geográfico acoplado con sus polinizadores; y c) analizar el nivel de congruencia entre las áreas seleccionadas con base en la distribución actual acoplada con sus polinizadores con respecto a los otros esquemas de conservación obtenidos.

\section{MATERIALES Y MÉTODOS}

\section{Área de estudio}

La unidad de estudio del presente capítulo fue la República Argentina, con una superficie de $3761274 \mathrm{~km}^{2}$. Está ubicada en el hemisferio sur con respecto al Ecuador y en el hemisferio occidental con relación al meridiano de Greenwich. Limita al norte con los países de Bolivia y Paraguay, al este con Brasil y Uruguay y al oeste con Chile.

\section{Especies bajo estudio}

El presente estudio incluyó 12 especies de cactáceas de la tribu Trichocereeae (Subfamilia Cactoideae) que viven en el noroeste de Argentina, de las cuales se 
conoce su biología reproductiva: Cleistocactus baumannii, C. smaragdiflorus, Denmoza rhodacantha, Echinopsis albispinosa, E. ancistrophora, E. atacamensis, E. haematantha, E. leucantha, E. schickendantzii, E. terscheckii, E. thelegona y Gymnocalycium saglionis. A fin de definir los rango acoplados para cada especie se utilizó la información relativa a los polinizadores (abejas, aves, avispas y mariposas nocturnas; Capítulos I y III).

\section{Selección de áreas prioritarias}

Para la selección de áreas prioritarias, los mapas de cada una de las 12 especies de cactáceas objeto de estudio se sobrepusieron sobre una grilla con celdas hexagonales de $53.11 \mathrm{~km} 2$ que abarca todo el territorio de Argentina a fin de obtener una matriz de presencia-ausencia de especies por celda. Se utilizó este tamaño de celda ya que corresponde a la mediana de la superficie que tienen las áreas protegidas en Argentina (aquellas categorizadas por IUCN de I a IV). Se utilizaron celdas hexagonales para minimizar el efecto de borde entre las mismas (Nhancale y Smith 2011). Con esta información se seleccionaron las áreas prioritarias para este grupo de especies utilizando el software MARXAN (Game y Grantham 2008). Esta es una herramienta que se utiliza para el diseño de sistemas de reservas basado en algoritmos heurísticos, y brinda una gran variedad de soluciones, casi óptimas, que pueden ser generadas con gran rapidez (Game y Grantham 2008). El objetivo es alcanzar una representación mínima de una meta cuantitativa específica al menor costo posible. Para el presente análisis, la meta de representación fue que el $10 \%$ del rango geográfico de cada una de las especies de cactáceas esté representado en el sistema de reservas elegido.

La selección de áreas prioritarias se realizó utilizando: 1) la distribución geográfica actual de las 12 especies de cactáceas, 2) la distribución geográfica que comparten con sus polinizadores (rango acoplado), 3) la distribución geográfica futura bajo el escenario RCP 4.5 para el año 2050 acoplada a sus polinizadores, 4) la distribución geográfica futura bajo el escenario RCP 4.5 para el año 2070 acoplada a sus polinizadores, 5) la distribución geográfica futura bajo el escenario RCP 8.5 para el año 2050 acoplada a sus polinizadores y 6) la distribución geográfica futura bajo el escenario RCP 8.5 para el año 2070 acoplada a sus polinizadores. En todos los casos, las distribuciones en escenarios 
climáticos futuros se hicieron bajo el supuesto de dispersión ilimitada (Capítulo III). Finalmente, se evaluó la congruencia espacial (expresada como el porcentaje de áreas seleccionadas, que son comunes a ambos esquemas de conservación) entre las áreas prioritarias seleccionadas a fin de evaluar de qué manera los esquemas de conservación seleccionados difieren de acuerdo al tipo de mapa de distribución utilizado en el presente (sin acople y con acople de polinizadores) y entre el presente y el futuro. Esta última comparación se realizó entre el esquema de conservación seleccionado con base en la distribución acoplada (con polinizadores) en el presente con respecto al esquema de conservación realizado con los mapas de distribución acoplados bajo los dos escenarios de cambio climático para los años 2050 y 2070. La comparación entre el sistema de áreas prioritarias del presente con respecto al futuro permitirá identificar las áreas comunes, las que de existir se consideran de máxima prioridad para la conservación de las especies bajo estudio y sus mutualistas. En todos los casos, la congruencia fue definida como el número de áreas seleccionadas como priotirarias compartidas por ambos esquemas de conservación, dividido por el número de áreas seleccionadas a partir del rango acoplado (considerando a los polinizadores) en el presente. Este valor se expresó en porcentaje, y representa el nivel de similitud entre los resultados de los esquemas de conservación bajo diferentes escenarios.

\section{RESULTADOS}

Teniendo en cuenta el rango geográfico de las especies de cactáceas sin sus polinizadores, se seleccionaron un total de 849 áreas prioritarias para el presente, distribuidas en 13 provincias (Fig. 1). Las provincias con mayor número de áreas seleccionadas fueron Salta (249), La Rioja (218) y Santiago del Estero (80). Se identificaron 600 áreas prioritarias para la conservación de las 12 especies de cactáceas incluidas en el análisis teniendo en cuenta el rango geográfico compartido con sus polinizadores en el presente (Fig. 2). Estas áreas estuvieron ubicadas en 12 provincias, concentrándose en Salta (235 áreas), La Rioja (104) y Tucumán (61). Del total de áreas seleccionadas teniendo en cuenta a las cactáceas sin sus polinizadores, 156 fueron compartidas con las seleccionadas 
teniendo en cuenta el rango acoplado, lo que representaría una congruencia del $26 \%$.

Bajo escenarios de cambio climático futuro, el número de áreas necesarias para cumplir la meta de conservación propuesta fue variable entre años y escenarios, alcanzando 440 y 377 áreas para el año 2050 (escenarios RCP 4.5 y 8.5, respectivamente), y 431 y 406 áreas para el año 2070 (escenarios RCP 4.5 y 8.5 , respectivamente). Para todos los escenarios futuros, las provincias en las cuales se seleccionó el mayor número de áreas fueron, en orden decreciente, Salta, La Rioja y Catamarca (Figs. 3-6). El nivel de congruencia entre las áreas seleccionadas como prioritarias para el presente y para el futuro en el año 2050 fue del $14.3 \%$ para el escenario RCP 4.5 (86 áreas compartidas con el presente) y del $15.5 \%$ para el escenario RCP 8.5 (93 áreas compartidas). Para el año 2070, el nivel de congruencia varió entre el 14.4\% para el escenario RCP 4.5 (87 áreas compartidas) y el 12.3\% para el escenario RCP 8.5 (74 áreas compartidas). Finalmente, se identificaron cinco áreas seleccionadas como prioritarias en el presente que también fueron seleccionadas para todos los escenarios de cambio climático (Fig. 7). Estas áreas se ubicaron en las provincias de Salta y La Rioja. De acuerdo a los mapas de distribución acoplados actuales, tres de ellas contienen a 11 de las 12 especies, y las restantes contienen 10 y seis especies.

\section{DISCUSIÓN}

Numerosos estudios resaltan la importancia de las interacciones bióticas en determinar el rango geográfico de una especie, aunque a un nivel jerárquico menor al de las condiciones climáticas o abióticas (Soberón 2007, Holland y Molina-Freaner 2012, Schweiger et al. 2012, Wisz et al. 2013). Sin embargo, muy pocos trabajos sobre planificación de estrategias de conservación tienen en cuenta a las interacciones que el grupo focal que se desea conservar establece con otros organismos (Giannini et al. 2013). Una manera de estimar el rango geográfico de una especie teniendo en cuenta a las interacciones, sería incluir sólo las áreas de su rango en las que también están presentes las especies con las que interactúa (rango acoplado, Capítulo III). De esta manera, se garantiza que en el esquema de conservación se incluya tanto al grupo focal como a las especies con las que interactúa y de las que eventualmente depende. Para el 
grupo de cactáceas estudiadas en el presente trabajo, se encontró un alto nivel de acople espacial entre el rango geográfico de las especies y el rango que las mismas comparten con sus polinizadores (Capítulo III). Por lo tanto, se esperaba que exista un alto nivel de congruencia entre las áreas prioritarias para la conservación seleccionadas a partir de la distribución de las cactáceas con las seleccionadas a partir de la distribución que comparten (acople espacial) con sus polinizadores. Sin embargo, los resultados indicaron un bajo nivel de congruencia (26\%), rechazando la hipótesis planteada. Esto quiere decir que las áreas prioritarias seleccionadas difieren en gran medida en función de considerar o no a las interacciones (en este caso, representadas como la presencia de los polinizadores). Dada la importancia de esta interacción mutualista para este grupo de plantas (Capítulo I), se considera que debería utilizarse el esquema de conservación que tiene en cuenta al rango geográfico acoplado a los polinizadores para la selección de áreas prioritarias. De esta manera, se seleccionarían áreas prioritarias que incluyen tanto a las especies bajo estudio como a los mutualistas con los que interactúa.

El número de áreas prioritarias seleccionadas para la conservación de las especies bajo estudio fue muy elevado (600 áreas teniendo en cuenta el rango acoplado). Esto se debe a que se planteó la meta de conservar el $10 \%$ del rango geográfico de cada especie, garantizando la representación de al menos una parte de la variabilidad genética que contienen las poblaciones a lo largo del rango geográfico. Sin embargo, generar acciones de conservación para este esquema de conservación parece difícil de alcanzar por el alto costo que esto representaría. Podrían plantearse otras metas de menor representación (por ejemplo, que cada especie esté representada una vez o dos veces en el esquema de conservación).

Bajo escenarios de cambio climático futuro, el número de áreas prioritarias seleccionadas (entre 377 y 440 áreas) fue menor con respecto al presente (600 áreas). Esto sería consecuencia de la contracción del rango geográfico en el futuro que se predijo para alguna de las especies, ya que la meta de representación consistió en un porcentaje del rango de cada especie, y se necesitaría un menor número de áreas para las especies que tendrán un rango más pequeño en el futuro. Las provincias de Salta y La Rioja fueron las que mayor número de áreas 
prioritarias presentaron para todos los escenarios futuros, así como para el presente. La importancia de la región noroeste del país para la conservación de cactáceas, y particularmente de la provincia de Salta, debido a sus altos niveles de riqueza y de endemismos ya ha sido señalada en trabajos anteriores (Ortega-Baes et al. 2012, 2015). En esta provincia, una alta proporción de áreas prioritarias se concentra en los Valles Calchaquíes, una de las regiones más diversas del país (Ortega-Baes et al. 2012).

Para todos los escenarios futuros, el nivel de congruencia de las áreas seleccionadas fue relativamente bajo (entre el 12.3 y el $15.5 \%$ ) con respecto a las áreas prioritarias seleccionadas para el presente. Es decir que una baja proporción de las áreas seleccionadas para el presente continuarían siendo prioritarias en el futuro. Dado que el cambio climático tiene el potencial de alterar la distribución geográfica de las especies, los efectos de este factor sobre la distribución de la diversidad deben ser tenidos en cuenta cuando se quieren establecer estrategias de conservación a largo plazo (Araújo et al. 2011). Particularmente, hubo cinco áreas prioritarias que fueron seleccionadas tanto en el presente como en los cuatro escenarios futuros, y deberían por lo tanto considerarse de máxima prioridad en un esquema de conservación para estas especies. La importancia de estas áreas radica en que son prioritarias para conservar a las cactáceas junto con sus mutualistas en la actualidad, y lo continuarán siendo en el futuro bajo escenarios de cambio climático. Cuatro de las cinco áreas de máxima prioridad se ubicaron en la provincia de Salta, y tres de ellas en los Valles Calchaquíes, señalados como uno de los sitios más diversos del país en riqueza de especies de esta familia de plantas (Ortega-Baes et al. 2012). El área restante correspondió a la provincia de La Rioja, que fue la segunda más importante en cuanto a número de áreas prioritarias tanto en el presente como en el futuro.

Las cactáceas son un grupo prioritario para acciones de conservación debido a que una gran proporción de especies se encuentra en peligro de extinción (Goettsch et al. 2015). La selección de áreas prioritarias para la conservación planteada en el presente trabajo constituye un punto de partida para la planificación sistemática de la conservación de este grupo de plantas. Si bien no se tuvo en cuenta al total de especies de la familia, y se planteó una meta de 
conservación que requeriría un elevado número de áreas prioritarias, es el primer esquema planteado teniendo en cuenta no sólo este grupo focal, sino también a los mutualistas de los cuales dependen las especies. Los resultados señalan que las áreas seleccionadas como prioritarias varían en gran medida si se tienen en cuenta estas interacciones, resaltando la importancia de la información acerca de la biología de las especies y sus interacciones, la cual es escasa y fragmentaria para este grupo de especies. En el caso del grupo bajo estudio, futuros estudios deberían incorporar en la definición de la distribución las interacciones positivas que se establecen para los procesos de dispersión de semillas (animales dispersores de semillas) y el establecimiento de plántulas (plantas nodrizas). Desde el punto de vista de la planificación para la conservación, los resultados ponen de manifiesto la necesidad de evaluar las cinco áreas consideradas como prioritarias a fin de que puedan ser incorporadas a esquemas de conservación para este grupo. Estas evaluaciones deberían incluir estudios sobre inventarios de la diversidad de cactáceas y de otros organismos que están presentes en esos sitios, las amenazas locales a las que se enfrenta dicha diversidad, estudios poblacionales, entre otros. Finalmente, los resultados destacan la importancia de incorporar las interacciones positivas en los esquemas de conservación in situ realizados para grupos prioritarios de plantas que como las cactáceas enfrentan altos niveles de amenaza en la actualidad, como es el caso de los árboles nativos de importancia forestal, las plantas de importancia ornamental (por ejemplo orquídeas) y las plantas de importancia aromático-medicinal. Esto requerirá, para estas especies, estudios comprensivos sobre las interacciones mutualistas que establecen con otros organismos.

\section{REFERENCIAS BIBLIOGRÁFICAS}

Andelman S.J., Willig M.R. (2002) Alternative configurations of conservation reserves for Paraguayan bats: considerations of spatial scale. Conservation Biology 16: 1352-1363.

Araújo, M.B., Luoto, M. (2007) The importance of biotic interactions for modelling species distributions under climate change. Global Ecology and Biogeography 16: 743-753.

Araújo M.B., Alagador D., Cabeza M., Nogués-Bravo D., Thuiller W. (2011) 
Climate change threatens European conservation areas. Ecology letters 14: 484-492.

Barnosky A.D., Matzke N., Tomiya S., Wogan G.O., Swartz B., Quental T.B., Marshall C., McGuire J.L., Lindsey E.L., Maguire K.C., Mersey B., Ferrer E.A. (2011) Has the Earth's sixth mass extinction already arrived? Nature 471: 51-57.

Bellard C., Bertelsmeier C., Leadley P., Thuiller W., Courchamp F. (2012) Impacts of climate change on the future of biodiversity. Ecology letters 15: 365-377.

Brooks T.M., Bakkarr M.I., Bouche T., Da Fonseca G.A.B., Hilton-Taylor C., Hoekstra J.M., Moritz T., Olivieri S., Parrish J., Pressey R., Rodrigues S.L., Sechrest W., Stattersfield A., Strahm W., Stuart S.N. (2004) Coverage provided by the global protected-area system: is it enough? Bioscience 54: 1081-1091.

Caldecott J.O., Jenkins M. D., Johnson T.H., Groombridge B. (1996) Priorities for conserving global species richness and endemism. Biodiversity and Conservation 5: 699-727.

Ceballos G., Ehrlich P.R. (2002) Mammal population losses and the extinction crisis. Science 296: 904-907.

Game E.T., Grantham H.S. (2008) Marxan User Manual: For Marxan version 1.8.10. University of Queensland, St. Lucia, Queensland, Australia, and Pacific Marine Analysis and Research Association, Vancouver, British Columbia, Canada.

Gaston K.J. (2000) Global patterns in biodiversity. Nature 405: 220-227.

Godínez-Alvarez H.O., Valverde T., Ortega-Baes P. (2003) Demographic trends in the Cactaceae. Botanical Review 69: 173-203.

Godoy-Bürki A.C., Aagesen L., Sajama J.M., Bravo S.A., Alonso-Pedano M., Ortega-Baes P. (2016) Patrones de rareza y prioridades de conservación en especies de Cactaceae del sur de los Andes Centrales: un estudio en los Valles Calchaquíes, Salta, Argentina. Ecología Austral 26: 72-82.

Goettsch B., Hilton-Taylor C., Cruz-Piñón G., Duffy J.P., Frances A., Hernández H.M., et al. (2015). High proportion of cactus species threatened with extinction. Nature plants 1: 15142. 
IPCC (2014) Cambio climático 2014: Informe de síntesis. Contribución de los Grupos de trabajo I, II y III al Quinto Informe de Evaluación del Grupo Intergubernamental de Expertos sobre el Cambio Climático [Equipo principal de redacción, R.K. Pachauri y L.A. Meyer (eds.)]. IPCC, Ginebra, Suiza, $157 \mathrm{p}$.

Margules C.R., Pressey R.L. (2000) Systematic conservation planning. Nature 405: 243-253.

Myers N. (2001) Hotsposts. En: Levin, S.A (Ed.) Enciclopedia of Biodiversity. San Diego Academics. pp. 387-381.

Nhancale B.A., Smith R.J. (2011) The influence of planning unit characteristics on the efficiency and spatial pattern of systematic conservation planning assessments. Biodiversity and Conservation 20: 1821-1835.

Ortega-Baes P., Shüring S., Sajama J., Sotola E., Alonso Pedano M., Bravo S., Godínez-Alvarez H. (2010) Diversity and conservation in the cactus family. En: Ramawat K. (ed). Desert Plants. Biology and Biotechnology. Springer.

Ortega-Baes, P., Bravo, S., Sajama, J., Sühring, S., Arrueta, J., Sotola, E., AlonsoPedano M., Godoy-Bürki A.C., Frizza N.R., Galíndez G. Gorostiague P., Barrionuevo A., Scopel A. (2012). Intensive field surveys in conservation planning: Priorities for cactus diversity in the Saltenian Calchaquíes Valleys (Argentina). Journal of arid environments 82: 91-97.

Ortega-Baes P.O, Godínez-Alvarez H., Sajama J., Gorostiague P., Sühring S., Galíndez G., Bravo S., López-Spahr D., Alonso-Pedano M., Lindow-López L., Barrionuevo A., Sosa C., Curti R.N., Juárez A. (2015). La familia Cactaceae en Argentina: patrones de diversidad y prioridades políticas para su conservación. Boletín de la Sociedad Argentina de Botánica 50: 71-78.

Parmesan C., Yohe G. (2003) A globally coherent fingerprint of climate change impacts across natural systems. Nature 421(6918): 37-42.

Parmesan C. (2006). Ecological and evolutionary responses to recent climate change. Annual Review of Ecology, Evolution, and Systematics 37: 637669.

Pimienta-Barrios E., del Castillo R.F. (2002) Reproductive biology. En: Nobel P.S. (ed.) Cacti: biology and uses. University of California Press. pp. 163-183. 
Pressey, R.L. (1994) Ad hoc reservations: forward or backward steps in developing representative reserve systems? Conservation Biology 8: 662-668.

Primack R.B. (2008) A Primer of Conservation Biology. Sinauer, Sunderland, USA. Rodrigues S.L., Akcakaya H.R., Andelman S.J., Bakarr M.I., Boitani L., Brooks T.M., Chanson J.S., Fishpool L.D.C., Da Fonseca G.A.B., Gaston K.J., Hoffman M., Long J.S., Marquet P.A., Pilgrim J.D., Pressey R.L., Schipper J., Sechrest W., Stuart SN, Underhill L.G., Waller R.W., Watts M.E., Yan X. (2004a) Global gap analysis: priority regions for expanding the global protected-area network. Bioscience 54: 1092-1100.

Rodrigues S.L., Andelman S.J., Bakarr M.I., Boitani L., Brooks T.M., Cowling R.M., Fishpool L.D.C., Da Fonseca G.A.B., Gaston K.J., Hoffman M., Long J.S., Marquet P.A., Pilgrim J.D., Pressey R.L., Schipper J., Sechrest W., Stuart SN, Underhill L.G., Waller R.W., Watts M.E., Yan X. (2004b) Effectiveness of global protected area network in representing species diversity. Nature 428: 640-643.

Soberón J. (2007) Grinnellian and Eltonian niches and geographic distributions of species. Ecology letters 10: 1115-1123.

Sodhi, N.S. (2010). Conservation biology for all. Oxford University Press.

Wisz M.S., Pottier J., Kissling W.D., Pellissier L., Lenoir J., Damgaard C.F., et al. (2013) The role of biotic interactions in shaping distributions and realised assemblages of species: implications for species distribution modelling. Biological Reviews 88(1): 15-30. 


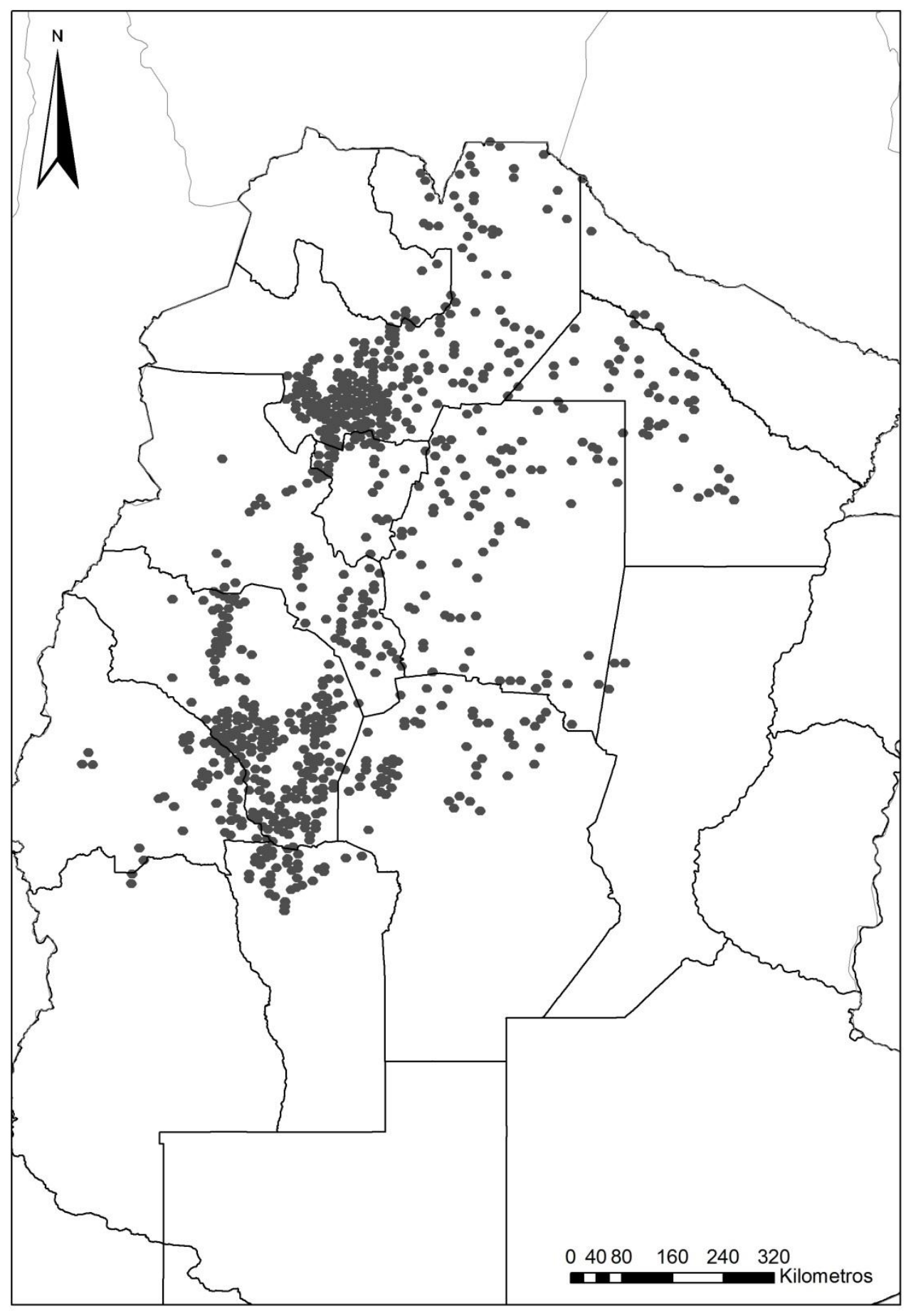

Figura 1. Áreas prioritarias seleccionadas a partir la distribución geográfica actual de las 12 especies de cactáceas incluidas en este estudio. 


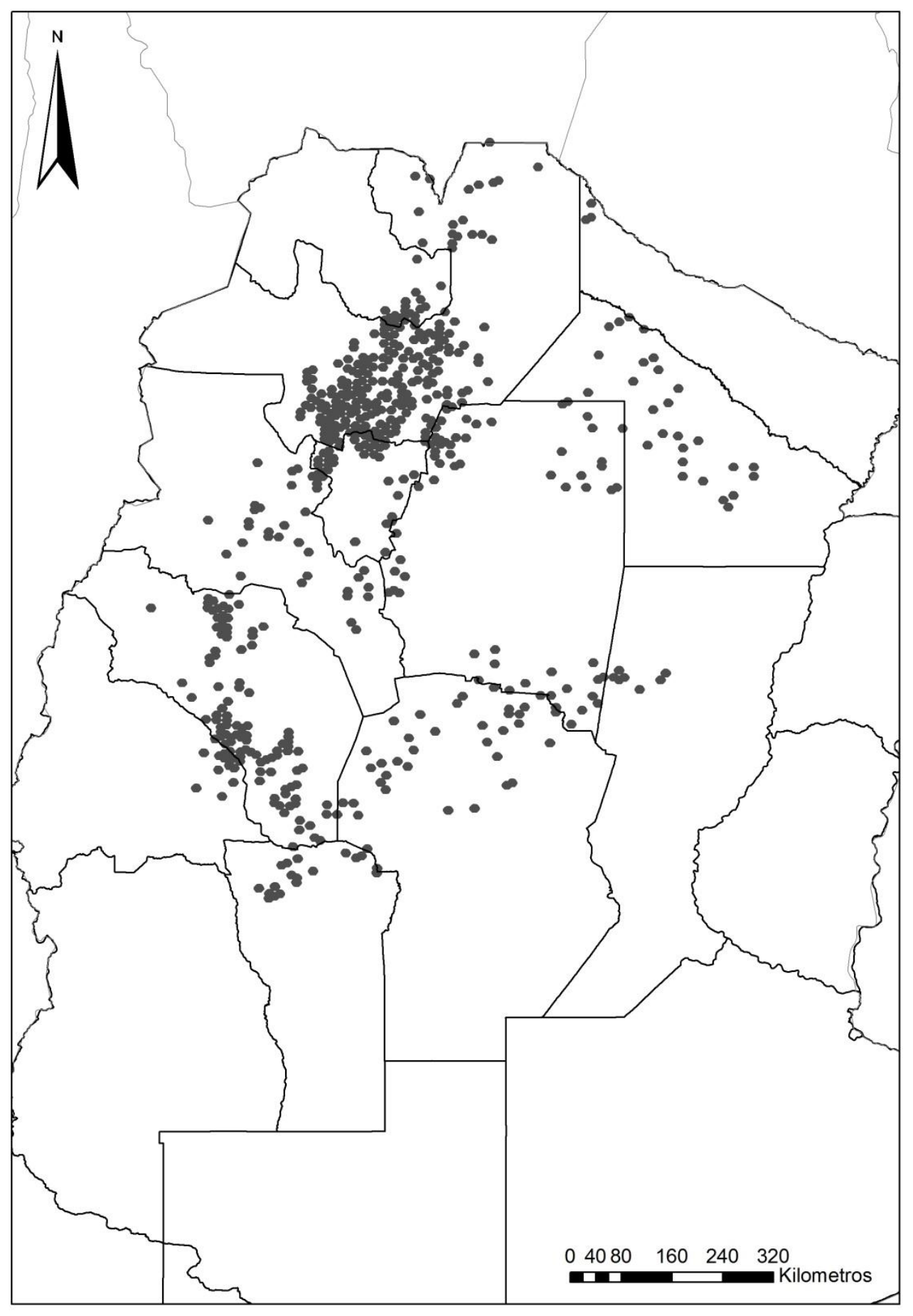

Figura 2. Áreas prioritarias seleccionadas a partir la distribución geográfica actual de las 12 especies de cactáceas incluidas en este estudio teniendo en cuenta el acople espacial con sus polinizadores. 


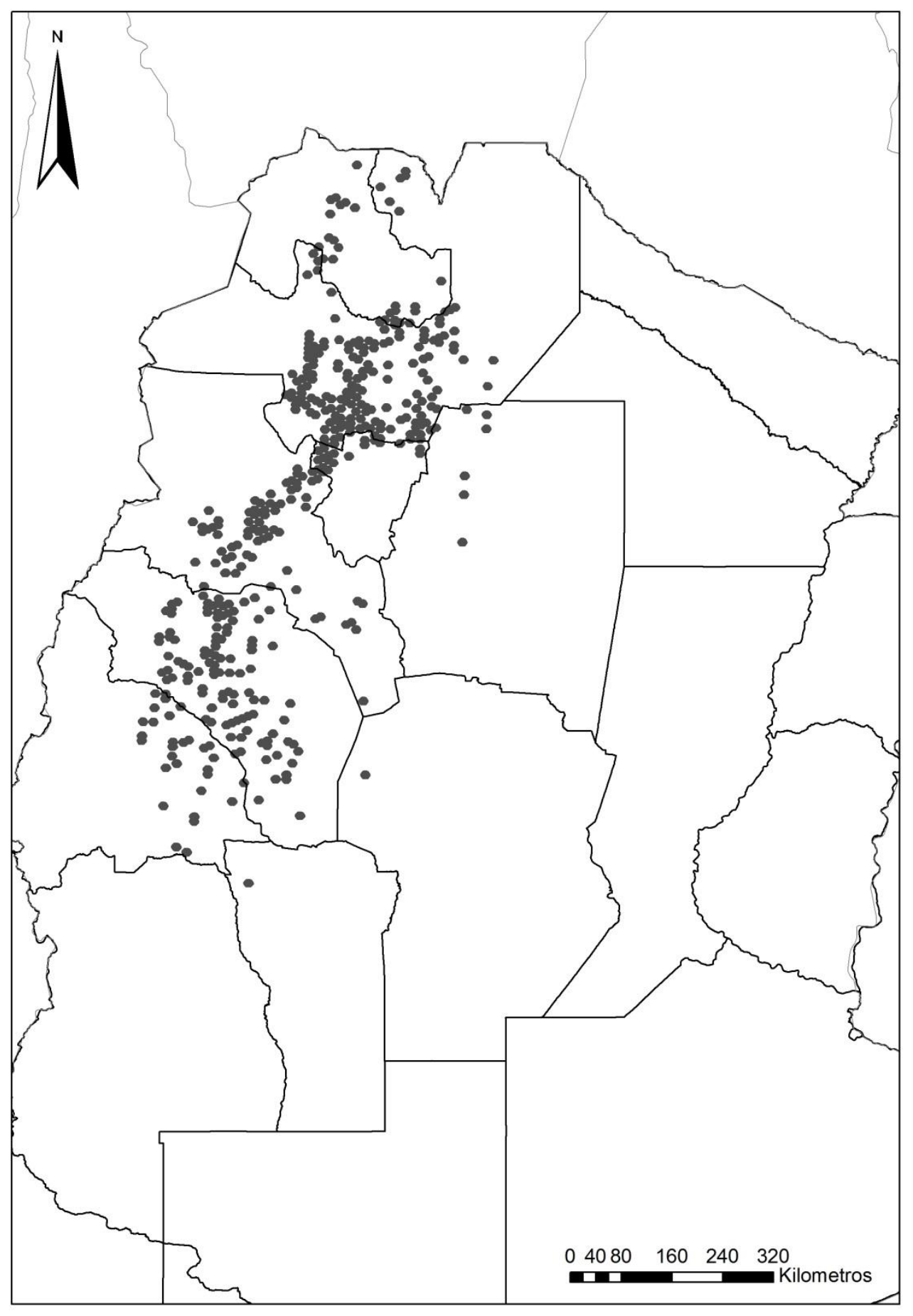

Figura 3. Áreas prioritarias seleccionadas a partir la distribución geográfica bajo el escenario de cambio climático RCP 4.5 para el año 2050 de las 12 especies de cactáceas incluidas en este estudio teniendo en cuenta el acople espacial con sus polinizadores. 


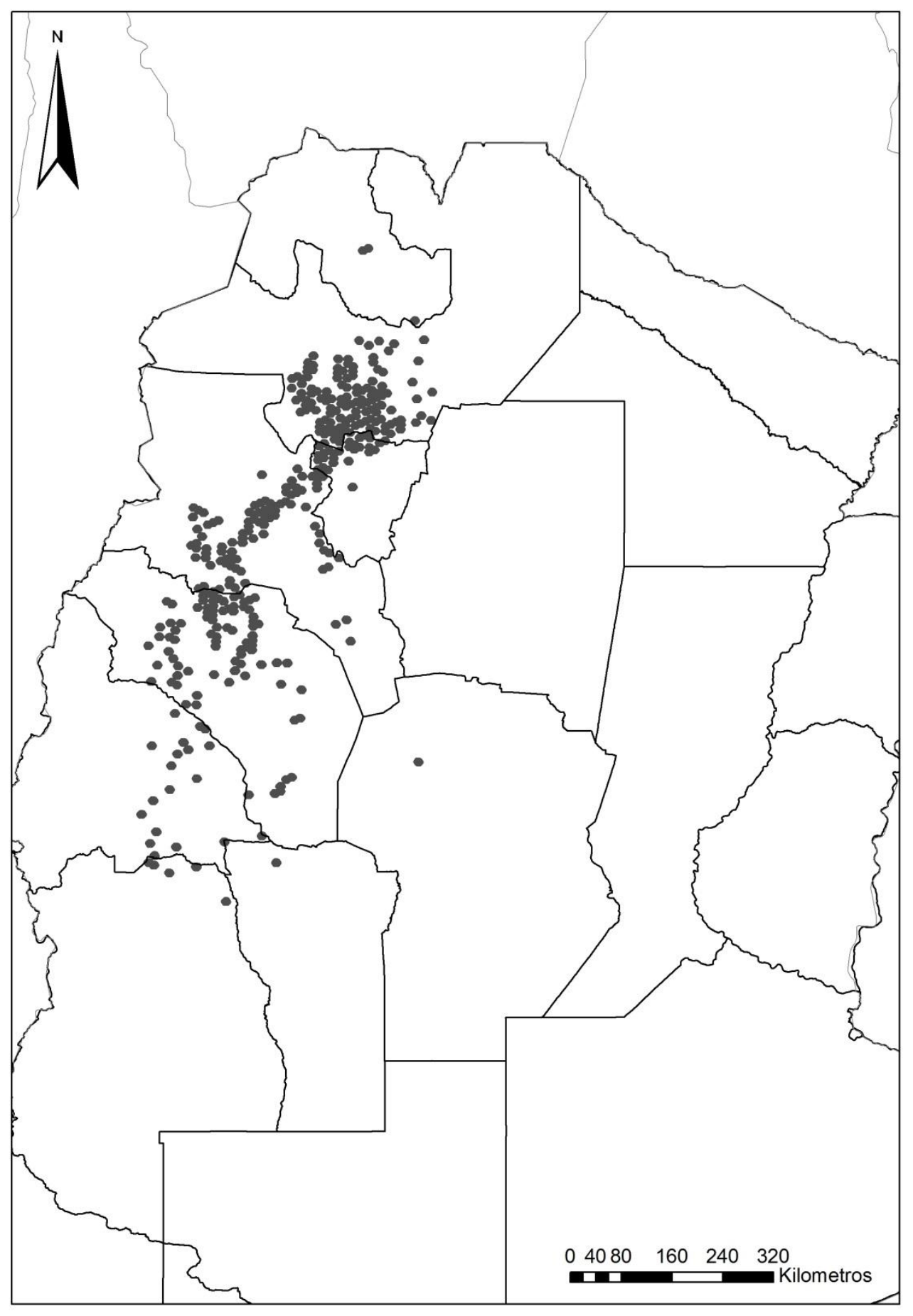

Figura 4. Áreas prioritarias seleccionadas a partir la distribución geográfica bajo el escenario de cambio climático RCP 8.5 para el año 2050 de las 12 especies de cactáceas incluidas en este estudio teniendo en cuenta el acople espacial con sus polinizadores. 


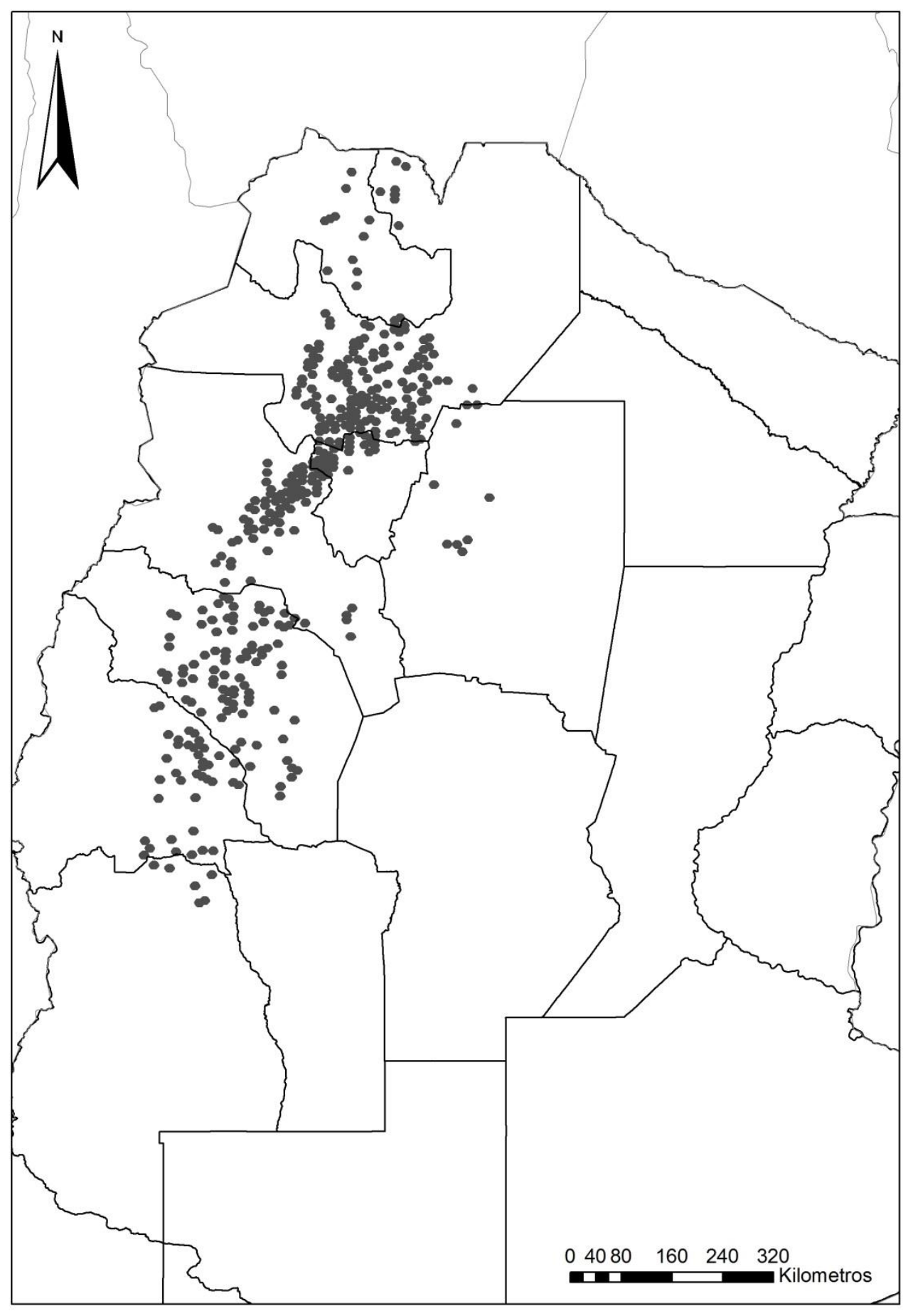

Figura 5. Áreas prioritarias seleccionadas a partir la distribución geográfica bajo el escenario de cambio climático RCP 4.5 para el año 2070 de las 12 especies de cactáceas incluidas en este estudio teniendo en cuenta el acople espacial con sus polinizadores. 


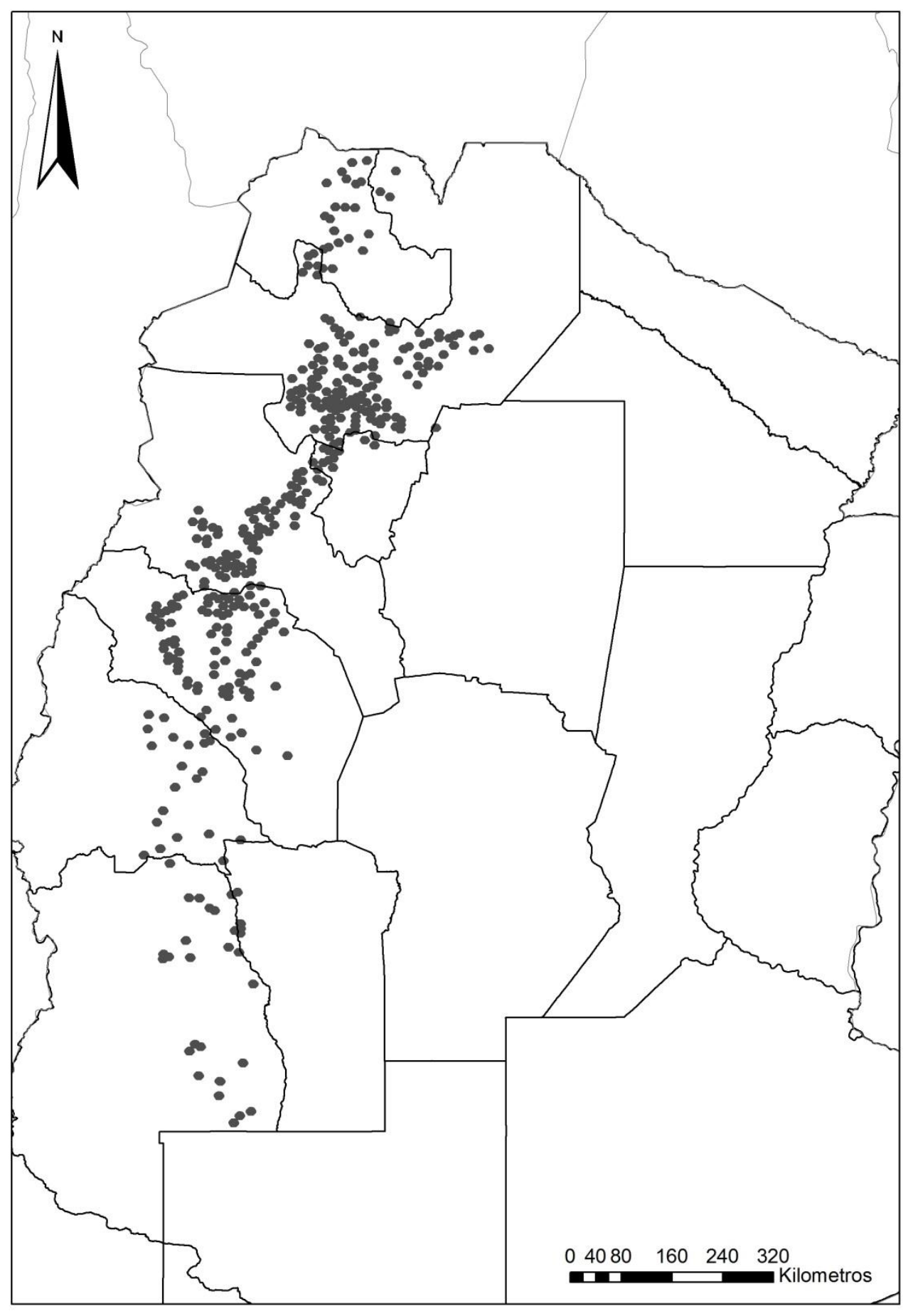

Figura 6. Áreas prioritarias seleccionadas a partir la distribución geográfica bajo el escenario de cambio climático RCP 8.5 para el año 2070 de las 12 especies de cactáceas incluidas en este estudio teniendo en cuenta el acople espacial con sus polinizadores. 


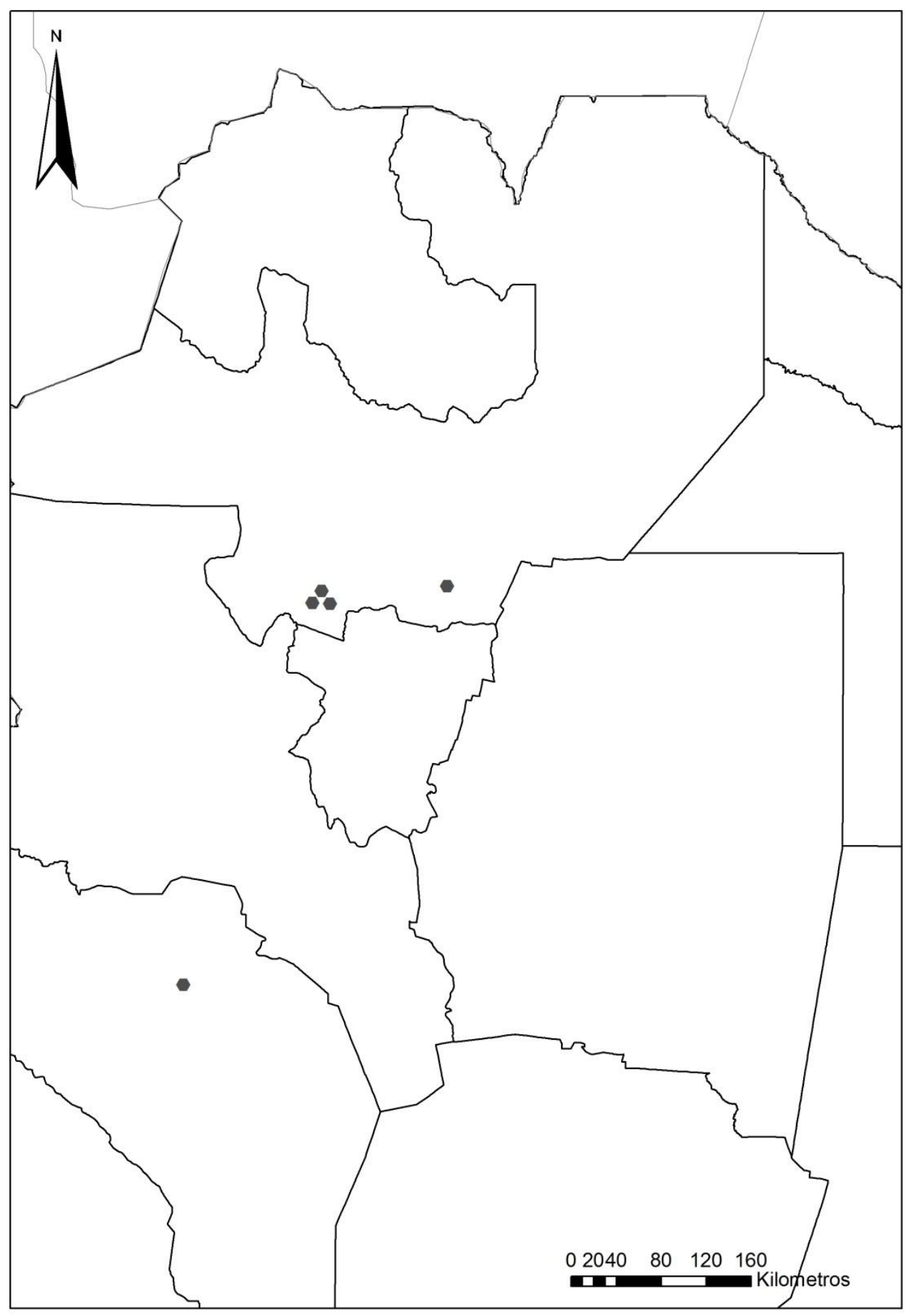

Figura 7. Áreas prioritarias seleccionadas a partir la distribución geográfica actual de las 12 especies de cactáceas incluidas en este estudio teniendo en cuenta el acople espacial con sus polinizadores que fueron compartidas por todos los escenarios de cambio climático futuro. 


\section{DISCUSIÓN GENERAL}

Los organismos no viven aislados en los sistemas naturales, sino que establecen numerosas interacciones con otras especies, de las cuales depende su distribución y abundancia. Algunas de estas interacciones representan un beneficio para las mismas, y en muchos casos son fundamentales para el desarrollo de sus ciclos de vida. Debido a que la biodiversidad que existe en la Tierra se encuentra amenazada como consecuencia de las actividades humanas, son necesarias estrategias de conservación tanto in situ como ex situ para evitar la pérdida de dicha diversidad (Primack 2008, Sodhi 2010). Tradicionalmente, estas estrategias de conservación se han concentrado sobre especies focales o grupos de especies emparentadas, sin tener en cuenta a las interacciones biológicas que éstas establecen con otros organismos. Dada la importancia de estas interacciones sobre la dinámica poblacional de algunas especies, resulta fundamental que los esquemas de conservación tengan en cuenta también a las especies de las cuales depende el grupo focal que se desea conservar.

Las cactáceas son un grupo de plantas que se consideran prioritarias para acciones de conservación. Se estima que el $31 \%$ de las especies de esta familia se encuentra en peligro de extinción debido, principalmente, a la degradación del hábitat y la colecta ilegal (Ortega-Baes et al.2010, Goesttsch et al. 2015). Adicionalmente, las cactáceas establecen numerosas interacciones mutualistas, de las cuales dependen para cumplir sus ciclos vitales, como la polinización, la dispersión de semillas y la facilitación para el establecimiento de nuevos individuos (Pimienta Barrios y del Castillo 2002, Godínez-Álvarez et al. 2003). Dado que los aspectos de la biología de las especies de este grupo de plantas han sido poco estudiados en nuestro país, se requieren esfuerzos para conocer el grado de dependencia de dichas interacciones, así como la planificación de estrategias de conservación para el grupo que tengan en cuenta a sus mutualistas.

De acuerdo a los resultados obtenidos en la presente tesis, las especies de cactáceas de la tribu Trichocereeae que viven en el noroeste de Argentina fueron auto-incompatibles, y por lo tanto, necesitan de sus polinizadores para la producción de frutos y semillas. Estos resultados coinciden con estudios previos que han destacado esta característica como común en las especies de la familia, 
incluidas especies que habitan en el noroeste de Argentina (Mandujano et al. 2010). Entre los animales polinizadores de las cactáceas de la tribu Trichocereeae del noroeste de Argentina, se registraron aves (picaflores y paseriformes) e insectos (abejas, avispas y mariposas nocturnas).

Tradicionalmente, se ha considerado que las especies de esta familia presentan sistemas de polinización especializados a grupos particulares de visitantes florales, que incluyen murciélagos, mariposas nocturnas, abejas, y aves. Para las especies estudiadas en la presente tesis, se registró especialización fenotípica (es decir, basada en las características florales) a mariposas nocturnas, abejas y aves. Las flores de estas especies pudieron ser clasificadas entre estos grupos con base en el color, tamaño y forma de la corola y el ciclo floral. Particularmente, para las flores ornitófilas, una evaluación para toda la familia indicó que el color rojo sería una característica que define la especialización fenotípica a este grupo de animales. Este último tipo de especialización es común entre las especies de la familia, y generalmente, está asociado a flores con forma tubular que abren durante el día. Sin embargo, a pesar de presentar especialización fenotípica en sus flores, las especies estudiadas presentaron mayoritariamente sistemas de polinización generalistas desde el punto de vista funcional o ecológico. En este sentido, sólo cuatro especies de las trece estudiadas presentaron sistemas de polinización especializados funcionalmente, dos a la polinización por abejas (E. haematantha y E. thionantha) y dos a la polinización por aves (C. baumannii y $D$. rhodacantha). La única especie con especialización ecológica fue $D$. rhodacantha, la cual es polinizada exclusivamente por el picaflor andino Oreotrochilus leucopleurus, sugiriendo que este tipo de especialización es raro en la familia Cactaceae.

Dado que las interacciones mutualistas tales como la polinización pueden limitar el rango geográfico que ocupan las especies de las cactáceas (Schweiger et al. 2010, 2012, Wisz et al. 2013), es fundamental conocer el grado de dependencia y la identidad de los mutualistas de cada especie, y tenerlas en cuenta al estimar su distribución geográfica. En esta tesis, se incorporaron las interacciones mutualistas de las cactáceas con sus polinizadores en la delimitación del rango geográfico de las especies, a través de la estimación del 
acople espacial. Los resultados indicaron, en la mayoría de los casos, que el rango geográfico de 12 especies de cactáceas analizadas presentaron un alto nivel de acople espacial con el rango de sus polinizadores. Además, se estimaron los efectos del cambio climático futuro sobre el rango geográfico de este grupo de cactáceas y sobre el acople espacial que existen entre estas especies y sus polinizadores. Los efectos del cambio climático sobre el rango geográfico de las especies de cactáceas estudiadas fueron positivos para algunas especies y negativos para otras. De acuerdo a los resultados obtenidos aquí, las especies que viven en zonas más áridas se verían beneficiadas con una expansión de su rango, mientas que las que habitan zonas semi-áridas se verían afectadas de manera negativa con una reducción en el tamaño de su rango geográfico. Debido a que el cambio climático podría tener efectos diferenciales sobre las cactáceas y las especies de animales que las polinizan, se esperaba que se genere una disrupción en esta interacción, expresado en un aumento en el desacople espacial entre los rangos de estas especies. Sin embargo, para la mayoría de los casos, los resultados indicaron que el cambio climático no generaría un aumento en el desacople espacial entre las cactáceas y sus polinizadores que presentan en la actualidad. Sólo una especie, la que posee el único sistema de polinización especializado ecológicamente (Denmoza rhodacantha), incrementaría su desacople espacial como consecuencia del cambio climático. Estos resultados ponen de manifiesto la necesidad de generar acciones de conservación para esta especie.

Si bien la mayoría de las especies de cactáceas presentaron un alto nivel de acople espacial con sus polinizadores en el presente, las áreas prioritarias para la conservación seleccionadas con base en el rango acoplado tuvieron una congruencia muy baja con respecto a las áreas seleccionadas con base en la distribución sin considerar los polinizadores. Este resultado resalta la importancia de tener en cuenta a las interacciones mutualistas en la estimación del rango geográfico de las especies, el cual constituye el insumo básico utilizado para la selección de áreas prioritarias para la conservación. Un esquema de conservación realizado con base en el rango acoplado entre las cactáceas y sus polinizadores garantizaría la presencia de ambos grupos de especies en las áreas 
seleccionadas como prioritarias para la conservación. Por otro lado, las áreas prioritarias seleccionadas bajo escenarios de cambio climático también presentaron una baja congruencia espacial con las áreas seleccionadas para el presente. Dado que el cambio climático tiene la capacidad de alterar los patrones de diversidad, es necesario tener en cuenta estos efectos para la planificación, en el presente, de estrategias de conservación a largo plazo. Con base en los resultados obtenidos, se proponen como prioritarias a cinco áreas que son comunes a todos los escenarios, tanto actuales como futuros, las cuales se ubican en las provincias de Salta y La Rioja. Estas áreas son de máxima prioridad para generar estrategias de conservación para las cactáceas y sus polinizadores. Futuros estudios deberían incorporar otras interacciones positivas que las cactáceas establecen con otras especies de plantas y animales (dispersores de semillas y plantas nodrizas) de manera de ajustar el rango geográfico de distribución a la porción de área en que las cactáceas no sólo disponen de hábitat disponible con base en factores del ambiente físico sino también de un hábitat en el que se encuentran con los organismos con los que establecen interacciones benéficas para el desarrollo de su ciclo de vida. Dado que muchos otros grupos de especies que tienen prioridad de conservación (por ejemplo especies de importancia forestal o especies de uso aromático-medicinal) establecen interacciones positivas con otros organismos, el enfoque desarrollado en la presente tesis debería ser incorporado a la hora de utilizar el rango geográfico para evaluar por un lado el impacto de factores de amenaza (por ejemplo del cambio en el uso del suelo o del cambio climático) y por el otro definir estrategias de conservación a largo plazo que garanticen la preservación de la biodiversidad objeto de conservación. Estos estudios deberán en primera instancia estudiar la biología de dichas interacciones, insumo básico para alcanzar el objetivo último que es el de generar acciones de conservación en el mundo real.

\section{REFERENCIAS BIBLIOGRÁFICAS}

Godínez-Alvarez H.O., Valverde T., Ortega-Baes P. (2003) Demographic trends in the Cactaceae. Botanical Review 69: 173-203.

Goettsch B., Hilton-Taylor C., Cruz-Piñón G., Duffy J.P., Frances A., Hernández H.M., et al. (2015). High proportion of cactus species threatened with 
extinction. Nature plants 1: 15142.

Mandujano M.C., Carrillo-Angeles I., Martínez-Peralta C., Golubov J. (2010)

Reproductive biology of Cactaceae. En: Ramawat K.J. (ed.) Desert Plants. Springer-Verlag, Berlin, Germany: 157-171.

Ortega-Baes P., Shüring S., Sajama J., Sotola E., Alonso Pedano M., Bravo S., Godínez-Alvarez H. (2010) Diversity and conservation in the cactus family. En: Ramawat K. (ed). Desert Plants. Biology and Biotechnology. Springer. Pimienta-Barrios E., del Castillo R.F. (2002) Reproductive biology. En: Nobel P.S. (ed.) Cacti: biology and uses. University of California Press. pp. 163-183.

Primack R.B. (2008) A primer of Conservation Biology. Sinauer. Sunderland, USA. Schweiger O., Biesmeijer J.C., Bommarco R., Hickler T., Hulme P.E., Klotz S., Kühn I., Moora M., Nielsen A., Ohlemüller R., Petanidou T., Potts S.G., Pysek P., Stout J.C., Sykes M.T., Tscheulin T., Vilà M., Walther G., Westphal C., Winter M., Zobel M., Settele J. (2010) Multiple stressors on biotic interactions: how climate change and alien species interact to affect pollination. Biological Reviews 85: 777-795.

Schweiger O., Heikkinen R.K., Harpke A., Hickler T., Klotz S., Kudrna O., Kühn I., Pöyry J., Settele J. (2012). Increasing range mismatching of interacting species under global change is related to their ecological characteristics. Global Ecology and Biogeography 21: 88-99.

Sodhi, N.S. (2010). Conservation biology for all. Oxford University Press.

Wisz M.S., Pottier J., Kissling W.D., Pellissier L., Lenoir J., Damgaard C.F., et al. (2013) The role of biotic interactions in shaping distributions and realised assemblages of species: implications for species distribution modelling. Biological Reviews 88(1): 15-30. 


\section{PUBLICACIONES}

Los siguientes trabajos fueron publicados a partir de los resultados presentados en esta Tesis:

Gorostiague P., Ortega-Baes P. (2016) How specialised is bird pollination in the Cactaceae? Plant Biology 18: 63-72. DOI: 10.1111/plb.12297.

Gorostiague P., Ortega-Baes P. (2017) Pollination biology of Echinopsis leucantha (Cactaceae): passerine birds and exotic bees as effective pollinators. Botany 95: 53-59. DOI: 10.1139/cjb-2016-0120. 